\title{
Modelo de cointegração variando com o tempo: Abordagem via ondaletas
}

\author{
Eder Lucio da Fonseca \\ TESE APRESENTADA \\ AO \\ Instituto DE Matemática e Estatística \\ DA \\ Universidade DE SÃo PaUlo \\ PARA \\ OBTENÇÃO DO TÍTULO \\ $\mathrm{DE}$ \\ DOUTOR EM CIÊNCIAS \\ Programa de Pós-Graduação em Estatística \\ Orientador: Profa. Dra. Airlane Pereira Alencar \\ Coorientador: Prof. Dr. Pedro Alberto Morettin
}

Durante o desenvolvimento deste trabalho o autor recebeu auxílio financeiro da CAPES.

São Paulo, Novembro de 2016. 


\section{Modelo de cointegração variando com o tempo: Abordagem via ondaletas}

\footnotetext{
Esta versão da tese de Eder Lucio da Fonseca contém as correções e alterações sugeridas pela Comissão Julgadora durante a defesa da versão original do trabalho, realizada em 06/03/2017. Uma cópia da versão original está disponível no Instituto de Matemática e Estatística da Universidade de São Paulo.
}

COMissão Julgadora:

- Profa. Dra. Airlane Pereira Alencar - IME-USP (Presidente)

- Prof. Dr. Pedro Alberto Morettin - IME-USP

- Profa. Dra. Thelma Safadi - UFLA

- Prof. Dr. Rogério de Faria Porto - Banco do Brasil

- Prof. Dr. Denisard Cneio de Oliveira Alves - FEA-USP 


\section{Agradecimentos}

Agradeço primeiramente a Deus, por sempre me dar conforto e esperança nos momentos em que mais precisei. Por sempre ouvir minhas preces e por ser um importante guia que impulsiona a minha vida. Agradeço por me trazer até aqui.

Agradeço carinhosamente meus pais Osvaldir Lucio da Fonseca e Sônia Aparecida Borges Fonseca, por apoiarem incondicionalmente os meus projetos de vida. Agradeço pelo amor, dedicação e zelo para com a nossa família. Agradeço principalmente por serem exemplos de caráter, honestidade e integridade. A eles todo o meu amor, respeito e gratidão.

Aos meus dois amados irmãos Eber Lucio da Fonseca e Franciele Borges Fonseca, por serem presenças constantes e queridas em minha vida. Agradecimento à minha cunhada Cristina Silva e à Yasmin Vitória Fonseca, minha querida sobrinha.

Aos meus queridos sogros Plínio Teixeira da Silva e Maria das Graças P. da Silva, por serem pessoas maravilhosas e por me tratarem como a um filho. Agradeço às minhas duas cunhadas/irmãs Adriana Almeida P. da Silva e Ângela P. da Silva (eterna Man) pelo apoio e pelas risadas despendidas gratuitamente.

Especial agradecimento à Andréa Almeida P. da Silva, amor da minha vida, por me apoiar em todos os momentos, incondicionalmente. Razão do meu viver, luz da minha vida! Agradeço também ao nosso cãozinho Malik, por continuar ao meu lado, sempre vigilante, principalmente nas solitárias madrugadas de estudo.

Agradeço à minha orientadora Airlane Pereira Alencar, por sempre me apoiar e contribuir para o meu crescimento científico, intelectual e profissional. Por sempre estar aberta ao diálogo e por sempre estar disposta a ouvir as minhas ideias. Por sempre poder contar com a sua ajuda. Agradeço pelo privilégio de ter sido seu orientado.

Aos Professores do IME Airlane Pereira Alencar, Pedro Alberto Morettin, Chang Chiann, Julio da Motta Singer, Silvia Nagib Elian e Carlos Alberto de Bragança Pereira (Carlinhos) por serem exemplos de conhecimento científico e excelência, fazendo jus ao grande instituto e à grande universidade que representam. A eles todo o meu respeito e admiração pelos profissionais que são.

Aos funcionários da secretaria de Pós-Graduação do IME, por toda a assistência prestada. À Universidade de São Paulo e ao IME, pela oportunidade e insumos fornecidos durante a realização do meu doutorado. À CAPES agradeço pelo apoio financeiro. 
Modelos são, em sua maior parte, caricaturas da realidade. Se eles são bons, então como boas caricaturas, eles retratam, embora de uma maneira distorcida, algumas das características do mundo real. O principal papel dos modelos não é apenas explicar e predizer - embora estas sejam as principais funções da ciência - é também polarizar o pensamento e fazer perguntas pertinentes.

Mark Kak, Some mathematical models in Science. 


\section{Resumo}

FONSECA, E. L. Modelo de cointegração variando com o tempo: Abordagem via ondaletas. 2016. 123 f. Tese (Doutorado) - Instituto de Matemática e Estatística, Universidade de São Paulo, São Paulo, 2016.

Duas ou mais séries não estacionárias são cointegradas se existir uma relação de equilíbrio de longo prazo entre elas. Nas últimas décadas, o interesse na literatura sobre o tema cointegração aumentou de maneira expressiva. Os modelos tradicionais supõem que o vetor de cointegração não varia ao longo do tempo. Entretanto, existem evidências na literatura de que esta suposição pode ser considerada muito restritiva. Utilizando o conceito de ondaletas, propomos um modelo de correção de erros vetorial em que é permitido ao vetor de cointegração variar ao longo do tempo. Diferente de trabalhos similares, é permitido ao vetor de cointegração variar suave ou abruptamente, dependendo da família de ondaletas considerada. Experimentos de Monte Carlo foram utilizados para estudar os quantis e o poder do teste de razão de verossimilhanças entre as hipóteses de cointegração usual e a de cointegração variando com o tempo. Os experimentos sugerem que o teste possui poder contra alternativas que variam ao longo do tempo. Foi demonstrada a capacidade do modelo em lidar satisfatoriamente com séries cointegradas simuladas, que apresentavam mudança de regime para o vetor de cointegração. O modelo foi empregado ainda para testar a validade da hipótese de paridade de poder de compra entre Estados Unidos e doze países da Organização para Cooperação e Desenvolvimento Econômico (OECD): Canadá, Japão e mais dez países europeus. Assim como em trabalhos similares, foram verificadas evidências de cointegração variando com o tempo entre os países. Foram utilizados valoresp bootstrap para verificar a significância da estatística do teste.

Palavras-chaves: Modelo Autorregressivo Vetorial Cointegrado (VAR), Modelo de Correção de Erros (VEC), Ondaletas, Cointegração Dinâmica, Paridade de Poder de Compra dinâmica, Métodos Bootstrap. 


\section{Abstract}

FONSECA, E. L. Time varying cointegration model: Approach using wavelets. 2016. 123 f. Doctoral Thesis - Instituto de Matemática e Estatística, Universidade de São Paulo, São Paulo, 2016.

Two or more non-stationary time series are cointegrated if there is a long-run equilibrium relationship between them. In recent decades, interest in the literature on the subject of cointegration increased expressively. Traditional models that address this issue assume that the cointegration vector does not vary over time. However, there is evidence in the literature that this assumption can be considered very restrictive. Using the concept of wavelets, we propose a vector error correction model in which is allowed to the cointegration vector vary over time. Unlike similar works, the cointegration vector is allowed to vary smoothly or abruptly, depending on the considered family of wavelets. Monte Carlo experiments were used to study the quantiles and the power of the likelihood ratio test of the hypotheses of usual cointegration versus the time-varying cointegration. The experiments suggest that the test has power against alternatives that vary over time. It was demonstrated the ability of the model to deal satisfactorily with simulated cointegrated series, which presented regime change for the cointegration vector. The model was also used to test the validity of the Purchasing Power Parity hypothesis between United States and twelve countries of the Organization for Economic Cooperation and Development (OECD): Canada, Japan and ten other European countries. As in similar works, evidence of time-varying cointegration was verified among countries. Bootstrap p-values were used to verify the significance of the likelihood ratio of the test.

Key-words: Cointegrated Vectorial Autoregressive Models (VAR), Error Correction Models $(V E C)$, Wavelets, Dynamic Cointegration, Dinamic Purchasing Power Parity, Bootstrap Methods. 


\section{Sumário}

Lista de Abreviaturas $\quad$ ix

Lista de Símbolos $\quad$ x

Lista de Figuras $\quad$ xi

Lista de Tabelas $\quad$ xvi

1 Introdução 1

2 Processos cointegrados e Modelo VEC 4

2.1 Processos cointegrados . . . . . . . . . . . . . . . . . . 4

2.2 Modelo VAR na forma de correção de erros . . . . . . . . . . . . . . . . . 7

2.3 Função de verossimilhança condicional . . . . . . . . . . . . . . . . . . . . 9

2.4 Estimadores de máxima verossimilhança . . . . . . . . . . . . . . . 11

2.5 Paridade de Poder de Compra . . . . . . . . . . . . . . . . 16

3 Representação de sinais utilizando ondaletas $\quad 19$

3.1 Ondaletas . . . . . . . . . . . . . . . . . . . . . . . . . 19

3.2 Famílias de ondaletas . . . . . . . . . . . . . . . . . . . . . . . 20

3.2.1 Família de ondaletas Haar . . . . . . . . . . . . . . . . . 20

3.2.2 Família de ondaletas Daublet . . . . . . . . . . . . . . . 22

3.2.3 Família de ondaletas Symmlet . . . . . . . . . . . . . . . . 23

3.3 Limiarização de ondaletas - contexto univariado . . . . . . . . . . . . . . 24

3.4 Limiarização de ondaletas - contexto multivariado . . . . . . . . . . . . 26

4 Modelo VEC variando com o tempo 29

4.1 Construção do modelo . . . . . . . . . . . . . . . . . . . . . . . . . 29

4.2 Função de verossimilhança condicional . . . . . . . . . . . . . . . . . 32

4.3 Estimadores de máxima verossimilhança . . . . . . . . . . . . . . 33

4.4 Construindo os estimadores de máxima verossimilhança dos parâmetros . . 38

4.4 .1 Estimadores normalizados . . . . . . . . . . . . . . . . 40

4.4.2 Caso especial - Modelo sem diferenças defasadas . . . . . . . . . . . 42

4.5 Teste de razão de verossimilhanças . . . . . . . . . . . . . . . . . 43 
4.6 Testes bootstrap - Propriedades empíricas dos estimadores . . . . . . . . . 44

4.7 Paridade de Poder de Compra variando com o tempo . . . . . . . . . . . . 46

4.7.1 Modelo desconsiderando o intercepto . . . . . . . . . . . . . 46

4.7.2 Modelo considerando o intercepto variando com o tempo e matriz de cointegração fixa . . . . . . . . . . . . . . . . . . 46 46

5 Relações de cointegração simuladas $\quad 51$

5.1 Quantis empíricos e poder empírico do teste de razão de verossimilhanças . 51

5.2 Exemplos sem defasagem . . . . . . . . . . . . . . . . 56

5.2.1 Primeiro exemplo: Inversão de sinal ao longo do tempo com Haar . 56

5.2.2 Segundo exemplo: Vetor de cointegração constante com Haar . . . . 63

5.2.3 Terceiro exemplo: Três escalas ao longo do tempo com Haar . . . . 68

5.2.4 Quarto exemplo: Decrescimento linear com Daubechies D2 . . . . . 73

5.2.5 Quinto exemplo: Transição suave entre duas escalas considerando limiarização e ondaleta Haar . . . . . . . . . . . . . . . . . . . . . . 79

5.3 Exemplo com defasagem . . . . . . . . . . . . . . . . . . . 90

5.3.1 Sexto exemplo: Decrescimento logarítmico com Symmlet $S 8$ (considerando defasagem do modelo) . . . . . . . . . . . . . . 90

6 Aplicação a dados reais $\quad 97$

6.1 PPC variando com o tempo - sem intercepto . . . . . . . . . . . . . . . 100

6.1.1 Ajuste com defasagem $p=13$ e ondaletas Daubechies D10 . . . . 109

$6.2 \mathrm{PPC}$ variando com o tempo - com intercepto . . . . . . . . . . . . . 114

7 Conclusão $\quad 116$

7.1 Considerações finais . . . . . . . . . . . . . . . . . . . 116

7.2 Pesquisas futuras . . . . . . . . . . . . . . . . . . . . . . . 118

$\begin{array}{ll}\text { A Resultados matriciais } & 119\end{array}$

B Demonstrações e passagens omissas no texto 120

B.1 Demonstrações do Capítulo 2 - Seção 2.3, Equação 2.27: ....... . 120

B.2 Tabelas longas do Capítulo $5 \ldots$. . . . . . . . . . . . . . . 121

B.2.1 Quarto exemplo: Decrescimento linear com Daubechies D2 . . . . . 121

B.2.2 Sexto exemplo: Decrescimento logarítmico com Symmlet S8 (considerando defasagem do modelo) . . . . . . . . . . . . . . . . . 124

$\begin{array}{ll}\text { C Elementos computacionais utilizados no trabalho } & 127\end{array}$

C.1 O software R e o pacote wavethresh . . . . . . . . . . . . 127

C.2 Rotina para simular modelos com cointegração variando com o tempo . . 127

$\begin{array}{ll}\text { Referências Bibliográficas } & 145\end{array}$ 


\section{Lista de Abreviaturas}

- VAR Modelo Autorregressivo Vetorial (Vector Autoregressive Model)

- VEC Modelo de Correção de Erros Vetorial (Vector Error Correction Model)

- PPC Paridade de Poder de Compra (Purchasing Power Parity - PPP)

- RCE Resíduos de cointegração estimados

- R.V. Razão de verossimilhanças

- v.a. Variáveis aleatórias

- i.i.d. Independentes e identicamente distribuídos

- Ex. Exemplo

- CVT Cointegração variando com o tempo 


\section{Lista de Símbolos}

$\begin{array}{ll}= & : \text { Igual } \\ := & : \text { Igual por definição } \\ \boldsymbol{\mu}_{t} & : \text { Vetor de médias para um processo multivariado não estacionário } \\ \gamma(t+\tau, t) & : \text { Matriz de covariâncias para um processo multivariado não estacionário } \\ \gamma_{i, j}(t+\tau, t) & : \text { Componentes da matriz } \gamma(t+\tau, t) \\ \boldsymbol{\mu} & : \text { Vetor de médias para um processo multivariado fracamente estacionário } \\ \gamma(\tau) & : \text { Matriz de covariâncias para um processo multivariado fracamente estacionário } \\ \gamma_{i, j}(\tau) & : \text { Componentes da matriz } \gamma(\tau) \\ \Delta & : \text { Operador diferença aplicado sobre uma série temporal } \\ \operatorname{tr}(\cdot) & : \text { Traço de uma matriz } \\ \rho(\cdot) & : \text { Posto de uma matriz } \\ |\cdot| & : \text { Determinante de uma matriz } \\ \boldsymbol{\alpha} & : \text { Matriz de carregamentos de um modelo VEC } \\ \boldsymbol{\beta} & : \text { Matriz de cointegração de um modelo VEC } \\ \boldsymbol{\Pi}:=\boldsymbol{\alpha} \boldsymbol{\beta}^{\prime} & : \text { Matriz } \Pi \text { de um modelo VEC } \\ \boldsymbol{\Gamma} & : \text { Matriz de coeficientes de curto prazo de um modelo VEC } \\ R V_{C V T}^{(J)} & : \text { Estatística do teste de R.V. para cointegração variando com o tempo }\end{array}$




\section{Lista de Figuras}

2.1 Esquema PPC teórica versus estimada. . . . . . . . . . . . . . . . . . . 18

3.1 Comportamento da ondaleta Haar pai $\phi_{00}(t)$ e mãe $\psi_{j k}(t)$, para $j=0,1$ e $2 \ldots$. 21

3.2 Comportamento da ondaleta Daublet D2 pai $\phi_{00}(t)$ e mãe $\psi_{j k}(t)$, para $j=0,1$ e 2.22

3.3 Comportamento da ondaleta Symmlet S4 pai $\phi_{00}(t)$ e mãe $\psi_{j k}(t)$, para $j=0,1$ e 2. 23

3.4 (a) Limiar duro (hard) $\operatorname{com} \lambda=1, \quad$ (b) Limiar suave (soft) $\operatorname{com} \lambda=1 \ldots$. . . 24

3.5 Exemplo de threshold universal para o contexto multivariado. Valores de $\theta_{j, k}$ abaixo do limiar devem ser excluídos do modelo. . . . . . . . . . . . . . . 28

4.1 Esquema PPC teórica invariante como tempo versus estimada variando com o

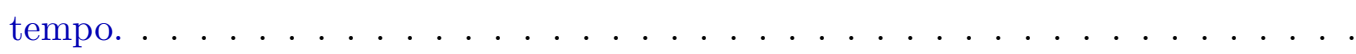

5.1 Primeiro exemplo: (a) Séries cointegradas simuladas $Y_{1 t}$ e $Y_{2 t}$, com $T=1024$, (b) Componente teórico $\beta_{2 t}^{*}$ de $\boldsymbol{\beta}_{t}^{*}=\left[\beta_{1 t}^{*} \beta_{2 t}^{*}\right]^{\prime}$ e estimado $\hat{\beta}_{2 t}^{*}$ de $\hat{\boldsymbol{\beta}}_{t}^{*}=\left[\hat{\beta}_{1 t}^{*} \hat{\beta}_{2 t}^{*}\right]^{\prime}$ e (c) Resíduos de cointegração teóricos $\left(\beta_{t}^{*^{\prime}} Y_{t-1}\right)$ e estimados $\left(\hat{\beta}_{t}^{*^{\prime}} Y_{t-1}\right)$. . . . . 58

5.2 Primeiro exemplo: Resíduos estimados pelo modelo, quantis amostrais e função de autocorrelação parcial (FACP) de $\hat{u}_{t}=\left[\hat{u}_{1 t}, \hat{u}_{2 t}\right]^{\prime} \ldots \ldots \ldots \ldots$. . . . . . . . 59

5.3 Primeiro exemplo: Histograma, densidade e quantis bootstrap 2, 5\% e 97, 5\% para $R V_{C V T}^{(J)}$ sob H0 (vetor de cointegração constante) (a) e sob HA (inversão de sinal ao longo do tempo) (b) . . . . . . . . . . . . . . . . .

5.4 Primeiro exemplo: Histograma, densidade e quantis bootstrap 2, 5\% e 97, 5\% sob HA (inversão de sinal ao longo do tempo) para as componentes $\hat{\alpha}_{11}^{*}$ (a) e $\hat{\alpha}_{21}^{*}$ (b)

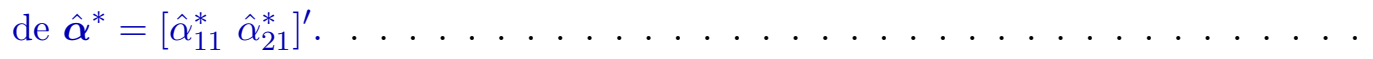

5.5 Primeiro exemplo: Histograma, densidade e quantis bootstrap 2,5\% e 97,5\% sob HA (inversão de sinal ao longo do tempo) para a componente $\hat{\sigma}_{11}^{*}$ (a), componentes $\hat{\sigma}_{12}^{*}$ e $\hat{\sigma}_{21}^{*}(\mathbf{b})$ e componente $\hat{\sigma}_{22}^{*}$ (c) de $\hat{\boldsymbol{\Sigma}}^{*} \ldots \ldots \ldots \ldots$

5.6 Primeiro exemplo: Histograma, densidade e quantis bootstrap 2, 5\% e 97, $5 \%$ sob HA (inversão de sinal ao longo do tempo) para as componentes $\hat{c}_{0,0}^{*(2,1)}$ (a) e $\hat{d}_{0,0}^{*(2,1)}$ (b) de $\hat{\boldsymbol{W}}_{J}^{*}=\left[\begin{array}{llll}c_{0,0}^{*(1,1)} & c_{0,0}^{*(2,1)} & d_{0,0}^{*(1,1)} & d_{0,0}^{*(2,1)}\end{array} \ldots \ldots \ldots \ldots \ldots \ldots \ldots\right.$

5.7 Segundo exemplo: (a) Séries cointegradas simuladas $Y_{1 t}$ e $Y_{2 t}$, $\operatorname{com} T=1024$, (b) Componente teórico $\beta_{2 t}^{*}$ de $\boldsymbol{\beta}_{t}^{*}=\left[\begin{array}{lll}\beta_{1 t}^{*} & \beta_{2 t}^{*}\end{array}\right]^{\prime}$ e estimado $\hat{\beta}_{2 t}^{*}$ de $\hat{\boldsymbol{\beta}}_{t}^{*}=\left[\begin{array}{ll}\hat{\beta}_{1 t}^{*} & \hat{\beta}_{2 t}^{*}\end{array}\right]^{\prime}$ e (c) Resíduos de cointegração teóricos $\left(\beta_{t}^{*^{\prime}} Y_{t-1}\right)$ e estimados $\left(\hat{\beta}_{t}^{*^{\prime}} Y_{t-1}\right) \ldots \ldots$. . . 
5.8 Segundo exemplo: Resíduos estimados pelo modelo, quantis amostrais e função de autocorrelação parcial (FACP) de $\hat{u}_{t}=\left[\hat{u}_{1 t}, \hat{u}_{2 t}\right]^{\prime}$.

5.9 Segundo exemplo: Histograma, densidade e quantis bootstrap 2,5\% e 97,5\% para $R V_{C V T}^{(J)}$ sob H0 (vetor de cointegração constante). . . . . . . . . . . . . .

5.10 Segundo exemplo: Histograma, densidade e quantis bootstrap 2,5\% e 97,5\% sob H0 (vetor de cointegração constante) para as componentes $\hat{\alpha}_{11}^{*}$ (a) e $\hat{\alpha}_{21}^{*}$ (b) de

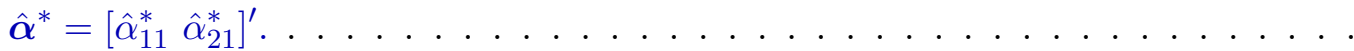

5.11 Segundo exemplo: Histograma, densidade e quantis bootstrap 2, 5\% e 97, $5 \%$ sob H0 (vetor de cointegração constante) para a componente $\hat{\sigma}_{11}^{*}$ (a), componentes $\hat{\sigma}_{12}^{*}$ e $\hat{\sigma}_{21}^{*}(\mathbf{b})$ e componente $\hat{\sigma}_{22}^{*}$ (c) de $\hat{\boldsymbol{\Sigma}}$. . . . . . . . . . . . . .

5.12 Segundo exemplo: Histograma, densidade e quantis bootstrap 2, 5\% e 97, 5\% sob H0 (vetor de cointegração constante) para as componentes $\hat{c}_{0,0}^{*(2,1)}$ (a) e $\hat{d}_{0,0}^{*(2,1)}$ (b) de $\hat{\boldsymbol{W}}_{J}=\left[\begin{array}{llll}c_{0,0}^{*(1,1)} & c_{0,0}^{*(2,1)} & d_{0,0}^{*(1,1)} & d_{0,0}^{*(2,1)}\end{array}\right] \ldots \ldots \ldots \ldots \ldots$

5.13 Terceiro exemplo: (a) Séries cointegradas simuladas $Y_{1 t}$ e $Y_{2 t}$, com $T=1024$, (b) componente teórico $\beta_{2 t}^{*}$ de $\boldsymbol{\beta}_{t}^{*}=\left[\beta_{1 t}^{*} \beta_{2 t}^{*}\right]^{\prime}$ e estimado $\hat{\beta}_{2 t}^{*}$ de $\hat{\boldsymbol{\beta}}_{t}^{*}=\left[\hat{\beta}_{1 t}^{*} \hat{\beta}_{2 t}^{*}\right]^{\prime}$, para $J=0$, (c) resíduos de cointegração teóricos $\left(\boldsymbol{\beta}_{t}^{*} Y_{t-1}\right)$ e estimados $\left(\hat{\boldsymbol{\beta}}_{t}^{*} Y_{t-1}\right)$ para $J=1$ e $(\mathbf{d})$ componente teórico $\beta_{2 t}^{*}$ de $\boldsymbol{\beta}_{t}^{*}=\left[\beta_{1 t}^{*} \beta_{2 t}^{*}\right]^{\prime}$ e estimado $\hat{\beta}_{2 t}^{*}$ de

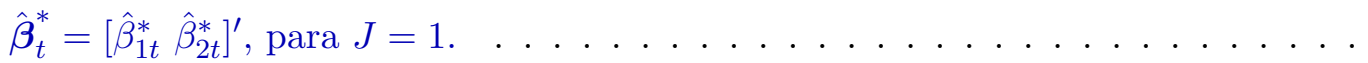

5.14 Terceiro exemplo: Resíduos estimados pelo modelo, quantis amostrais e função de autocorrelação parcial (FACP) de $\hat{u}_{t}=\left[\hat{u}_{1 t}, \hat{u}_{2 t}\right]^{\prime} \ldots \ldots \ldots \ldots \ldots$

5.15 Terceiro exemplo: Histograma, densidade e quantis bootstrap 2, $5 \%$ e 97,5\% para $R V_{C V T}^{(J)}$ sob H0 (vetor de cointegração constante) (a) e sob HA (três escalas ao longo do tempo) (b) . . . . . . . . . . . . . . . . . . . . .

5.16 Terceiro exemplo: Histograma, densidade e quantis bootstrap 2,5\% e 97,5\% sob HA (três escalas ao longo do tempo) para as componentes $\hat{\alpha}_{11}^{*}$ (a) e $\hat{\alpha}_{21}^{*}$ (b) de

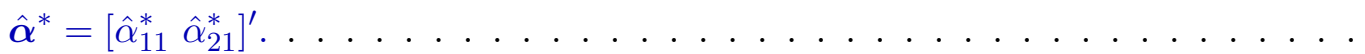

5.17 Terceiro exemplo: Histograma, densidade e quantis bootstrap 2, 5\% e 97, $5 \%$ sob HA (três escalas ao longo do tempo) para a componente $\hat{\sigma}_{11}^{*}$ (a), componentes $\hat{\sigma}_{12}^{*}$ e $\hat{\sigma}_{21}^{*}(\mathbf{b})$ e componente $\hat{\sigma}_{22}^{*}$ (c) de $\hat{\boldsymbol{\Sigma}}^{*}$.

5.18 Terceiro exemplo: Histograma, densidade e quantis bootstrap 2,5\% e 97,5\% sob HA (três escalas ao longo do tempo) para as componentes $\hat{c}_{0,0}^{*(2,1)}$ (a) e $\hat{d}_{0,0}^{*(2,1)}$

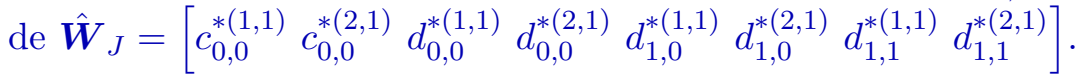

5.18 Terceiro exemplo (continuação): Histograma, densidade e quantis bootstrap 2, 5\% e $97,5 \%$ sob HA (três escalas ao longo do tempo) para as componentes $\hat{d}_{1,0}^{*(2,1)}$ (c)

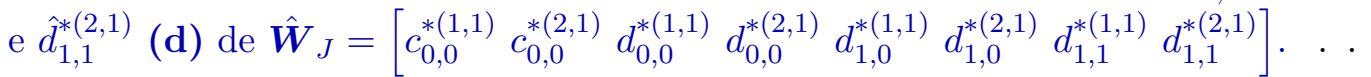

5.19 Quarto exemplo: (a) Séries cointegradas simuladas $Y_{1 t}$ e $Y_{2 t}$, com $T=1024$, (b) Componente teórico $\beta_{2 t}^{*}$ de $\boldsymbol{\beta}_{t}^{*}=\left[\begin{array}{ll}\beta_{1 t}^{*} & \beta_{2 t}^{*}\end{array}\right]^{\prime}$ e estimado $\hat{\beta}_{2 t}^{*}$ de $\hat{\boldsymbol{\beta}}_{t}^{*}=\left[\begin{array}{ll}\hat{\beta}_{1 t}^{*} & \hat{\beta}_{2 t}^{*}\end{array}\right]^{\prime}$ e (c) Resíduos de cointegração teóricos $\left(\beta_{t}^{*^{\prime}} Y_{t-1}\right)$ e estimados $\left(\hat{\beta}_{t}^{*^{\prime}} Y_{t-1}\right)$. . . . . .

5.20 Quarto exemplo: Resíduos estimados pelo modelo, quantis amostrais e função de autocorrelação parcial (FACP) de $\hat{u}_{t}=\left[\hat{u}_{1 t}, \hat{u}_{2 t}\right]^{\prime}$. 
5.21 Quarto exemplo: Histograma, densidade e quantis bootstrap 2, 5\% e 97,5\% para $R V_{C V T}^{(J)}$ sob H0 (vetor de cointegração constante) (a) e sob HA (decrescimento

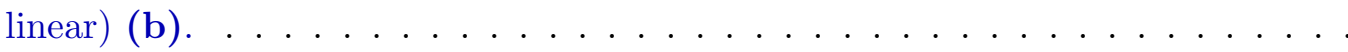

5.22 Quarto exemplo: Histograma, densidade e quantis bootstrap 2, 5\% e 97, $5 \%$ sob HA (decrescimento linear) para as componentes $\hat{\alpha}_{11}^{*}(\mathbf{a})$ e $\hat{\alpha}_{21}^{*}(\mathbf{b})$ de $\hat{\boldsymbol{\alpha}}^{*}=\left[\begin{array}{ll}\hat{\alpha}_{11}^{*} & \hat{\alpha}_{21}^{*}\end{array}\right]^{\prime} \cdot 76$

5.23 Quarto exemplo: Histograma, densidade e quantis bootstrap 2,5\% e 97,5\% sob HA (decrescimento linear) para a componente $\hat{\sigma}_{11}^{*}(\mathbf{a})$, componentes $\hat{\sigma}_{12}^{*}$ e $\hat{\sigma}_{21}^{*}(\mathbf{b})$ e componente $\hat{\sigma}_{22}^{*}$ (c) de $\hat{\boldsymbol{\Sigma}}^{*} \ldots \ldots \ldots \ldots \ldots \ldots$

5.24 Quinto exemplo: (a) Séries cointegradas simuladas $Y_{1 t}$ e $Y_{2 t}$, com $T=1024$, (b) componente teórico $\beta_{2 t}$ de $\boldsymbol{\beta}_{t}=\left[\beta_{1 t} \text { e } \beta_{2 t}\right]^{\prime}$ e estimado $\hat{\beta}_{2 t}$ de $\hat{\boldsymbol{\beta}}_{t}=\left[\hat{\beta}_{1 t} \hat{\beta}_{2 t}\right]^{\prime}$. . . .

5.24 Quinto exemplo (continuação): (c) Resíduos de cointegração teóricos $\left(\boldsymbol{\beta}_{t}^{\prime} Y_{t-1}\right)$ e

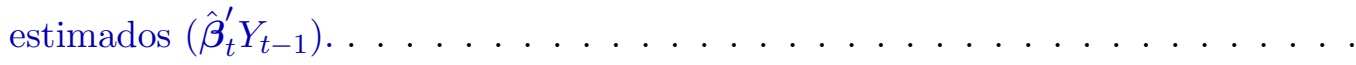

5.25 Quinto exemplo (completo): Resíduos estimados pelo modelo, quantis amostrais e função de autocorrelação parcial (FACP) de $\hat{u}_{t}=\left[\hat{u}_{1 t}, \hat{u}_{2 t}\right]^{\prime} \ldots \ldots \ldots$. . . . .

5.26 Quinto exemplo: Histograma, densidade e quantis bootstrap 2,5\% e 97,5\% para $R V_{C V T}^{(J)}$ sob H0 (transição suave entre duas escalas) (a) e sob HA (transição suave entre dois regimes) $(\mathbf{b}) . \ldots \ldots \ldots \ldots \ldots$

5.27 Quinto exemplo: Histograma, densidade e quantis bootstrap 2,5\% e 97, 5\% sob HA (transição suave entre duas escalas) para as componentes $\hat{\alpha}_{11}$ (a) e $\hat{\alpha}_{21}$ (b) de $\hat{\boldsymbol{\alpha}}=\left[\begin{array}{ll}\hat{\alpha}_{11} & \hat{\alpha}_{21}\end{array}\right]^{\prime} \ldots \ldots \ldots \ldots \ldots \ldots \ldots \ldots \ldots$

5.28 Quinto exemplo: Histograma, densidade e quantis bootstrap 2, 5\% e 97, $5 \%$ sob HA (transição suave entre duas escalas) para a componente $\hat{\sigma}_{11}$ (a), componentes $\hat{\sigma}_{12}$ e $\hat{\sigma}_{21}(\mathbf{b})$ e componente $\hat{\sigma}_{22}(\mathbf{c})$ de $\hat{\boldsymbol{\Sigma}}$. . . . . . . . . . . . . . . 83

5.29 Threshold para o modelo completo, considerando $J=3 \ldots \ldots$. . . . . . . . 84

5.30 Componente $\hat{\beta}_{2 t}$ de $\hat{\beta}_{t}=\left(\hat{\beta}_{1 t}, \hat{\beta}_{2 t}\right)$ e teórico $\beta_{2 t}$ para referência: Modelos 01, 02,

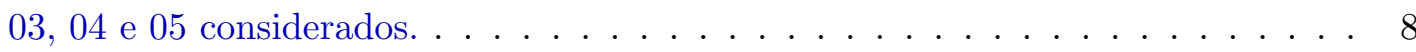

5.31 (a) Componente $\hat{\beta}_{2 t}$ de $\hat{\beta}_{t}=\left(\hat{\beta}_{1 t}, \hat{\beta}_{2 t}\right)$ e teórico $\beta_{2 t}$ para referência $(\mathrm{J}=3), \quad(\mathbf{b})$ Resíduos de cointegração teóricos $\left(\beta_{t}^{\prime} Y_{t-1}\right)$ e estimados $\left(\hat{\beta}_{t}^{\prime} Y_{t-1}\right) \ldots \ldots \ldots$. . . 86

5.32 Quinto exemplo (limiarizado): Resíduos estimados pelo modelo, quantis amostrais e função de autocorrelação parcial (FACP) de $\hat{u}_{t}=\left[\hat{u}_{1 t}, \hat{u}_{2 t}\right]^{\prime}$. . . . . . . . . .

5.33 Quinto exemplo (limiarizado): Histograma, densidade e quantis bootstrap 2,5\% e $97,5 \%$ para $R V_{C V T}^{(J)}$ sob H0 (vetor de cointegração constante) (a) e sob HA (transição suave entre dois regimes) $(\mathbf{b}) \ldots \ldots \ldots \ldots$. . . . . . . . . .

5.34 Quinto exemplo (limiarizado): Histograma, densidade e quantis bootstrap 2,5\% e $97,5 \%$ para as componentes $\hat{\alpha}_{11}$ (a) e $\hat{\alpha}_{21}$ (b) de $\hat{\boldsymbol{\alpha}}=\left[\hat{\alpha}_{11} \hat{\alpha}_{21}\right]^{\prime}$. . . . . . . .

5.35 Quinto exemplo (limiarizado): Histograma, densidade e quantis bootstrap 2,5\% e $97,5 \%$ sob HA (transição suave entre duas escalas) para a componente $\hat{\sigma}_{11}$ (a), componentes $\hat{\sigma}_{12}$ e $\hat{\sigma}_{21}$ (b) e componente $\hat{\sigma}_{22}$ (c) de $\hat{\boldsymbol{\Sigma}}$. . . . . . . . . . . .

5.36 Sexto exemplo: (a) Séries cointegradas simuladas $Y_{1 t}$ e $Y_{2 t}$, com $T=1024$, (b) Componente teórico $\beta_{2 t}^{*}$ de $\boldsymbol{\beta}_{t}^{*}=\left[\begin{array}{ll}\beta_{1 t}^{*} & \beta_{2 t}^{*}\end{array}\right]^{\prime}$ e estimado $\hat{\beta}_{2 t}^{*}$ de $\hat{\boldsymbol{\beta}}_{t}^{*}=\left[\begin{array}{ll}\hat{\beta}_{1 t}^{*} & \hat{\beta}_{2 t}^{*}\end{array}\right]^{\prime}$ e (c) Resíduos de cointegração teóricos $\left(\beta_{t}^{*^{\prime}} Y_{t-1}\right)$ e estimados $\left(\hat{\beta}_{t}^{*^{\prime}} Y_{t-1}\right)$. . . . . . 
5.37 Quarto exemplo: Resíduos estimados pelo modelo, quantis amostrais e função de autocorrelação parcial $(\mathrm{FACP})$ de $\hat{u}_{t}=\left[\hat{u}_{1 t}, \hat{u}_{2 t}\right]^{\prime} \ldots \ldots \ldots \ldots$. . . . . . .

5.38 Sexto exemplo: Histograma, densidade e quantis bootstrap 2,5\% e 97,5\% para $R V_{C V T}^{(J)}$ sob H0 (vetor de cointegração constante) (a) e sob HA (decrescimento

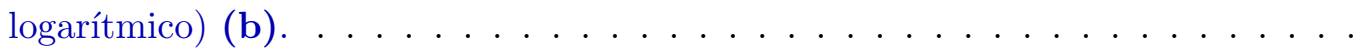

5.39 Sexto exemplo: Histograma, densidade e quantis bootstrap 2,5\% e 97,5\% sob HA (decrescimento logarítmico) para as componentes $\hat{\alpha}_{11}^{*}(\mathbf{a})$ e $\hat{\alpha}_{21}^{*}(\mathbf{b})$ de $\hat{\boldsymbol{\alpha}}^{*}=$

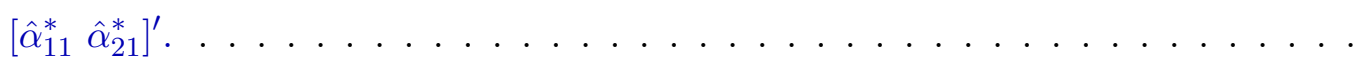

5.40 Sexto exemplo: Histograma, densidade e quantis bootstrap 2, 5\% e 97, 5\% sob HA (decrescimento logarítmico) para a componente $\hat{\sigma}_{11}^{*}(\mathbf{a})$, componentes $\hat{\sigma}_{12}^{*}$ e $\hat{\sigma}_{21}^{*}$ (b) e componente $\hat{\sigma}_{22}^{*}$ (c) de $\hat{\boldsymbol{\Sigma}}^{*} \ldots \ldots \ldots \ldots \ldots$

5.41 Sexto exemplo: Histograma, densidade e quantis bootstrap 2, 5\% e 97, $5 \%$ sob HA (decrescimento logarítmico)para as componentes $\hat{\Gamma}_{11}^{*(1)}$ (a) e $\hat{\Gamma}_{12}^{*(1)}$ (b) de $\hat{\Gamma}_{\mathbf{1}}^{*}$. . .

5.41 Sexto exemplo (continuação): Histograma, densidade e quantis bootstrap 2, 5\% e $97,5 \%$ sob HA (decrescimento logarítmico)para as componentes $\hat{\Gamma}_{21}^{*(1)}$ (c) e $\hat{\Gamma}_{22}^{*(1)}$

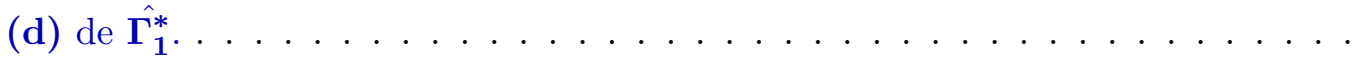

6.1 Gráficos com índices de preços dos EUA (país local) e dos países estrangeiros Bélgica, Canadá, Dinamarca, França, Alemanha, Itália, Japão e Holanda, juntamente com as respectivas taxas de câmbio. . . . . . . . . . . . . .

6.1 (continuação) Gráficos com índices de preços dos EUA (país local) e dos países estrangeiros Noruega, Espanha, Suécia e Reino Unido, juntamente com as respectivas taxas de câmbio. . . . . . . . . . . . . . . . . . . . . . 100

6.2 PPC entre Estados Unidos e Bélgica, tomando $J=0$ : Componentes $\hat{\beta}_{1 t}$ (a), $\hat{\beta}_{2 t}$ (b) e $\hat{\beta}_{3 t}$ (c) de $\hat{\boldsymbol{\beta}}_{t}=\left[\begin{array}{lll}\hat{\beta}_{1 t} & \hat{\beta}_{2 t} & \hat{\beta}_{3 t}\end{array}\right]^{\prime}$ juntamente com bandas bootstrap de $95 \%$. Para efeito comparativo, os três componentes são apresentadas no mesmo gráfico (d) . . . . . . . . . . . . . . . . . . . . . . 106

6.3 PPC entre Estados Unidos e Japão, tomando $J=1$ : Componentes $\hat{\beta}_{1 t}$ (a), $\hat{\beta}_{2 t}$ (b) e $\hat{\beta}_{3 t}$ (c) de $\hat{\boldsymbol{\beta}}_{t}=\left[\begin{array}{lll}\hat{\beta}_{1 t} & \hat{\beta}_{2 t} & \hat{\beta}_{3 t}\end{array}\right]^{\prime}$ juntamente com bandas bootstrap de $95 \%$. Para efeito comparativo, os três componentes são apresentadas no mesmo gráfico (d) . . . . . . . . . . . . . . . . . . . . . . 106

6.4 PPC entre Estados Unidos e Noruega, tomando $J=2$ : Componentes $\hat{\beta}_{1 t}$ (a), $\hat{\beta}_{2 t}$ (b) e $\hat{\beta}_{3 t}$ (c) de $\hat{\boldsymbol{\beta}}_{t}=\left[\hat{\beta}_{1 t} \hat{\beta}_{2 t} \hat{\beta}_{3 t}\right]^{\prime}$ juntamente com bandas bootstrap de $95 \%$. Para efeito comparativo, os três componentes são apresentadas no mesmo gráfico (d) . . . . . . . . . . . . . . . . . . . . 107

6.5 PPC entre Estados Unidos e Alemanha, tomando $J=3$ : Componentes $\hat{\beta}_{1 t}$ (a), $\hat{\beta}_{2 t}(\mathrm{~b})$ e $\hat{\beta}_{3 t}$ (c) de $\hat{\boldsymbol{\beta}}_{t}=\left[\hat{\beta}_{1 t} \hat{\beta}_{2 t} \hat{\beta}_{3 t}\right]^{\prime}$ juntamente com bandas bootstrap de $95 \%$. Para efeito comparativo, os três componentes são apresentadas no mesmo gráfico (d) . . . . . . . . . . . . . . . . . . . . 107

6.6 PPC entre Estados Unidos e Canadá: Resíduos estimados pelo modelo, quantis amostrais e função de autocorrelação parcial (FACP) de $\hat{u}_{t}=\left[\begin{array}{llll}\hat{u}_{1 t} & \hat{u}_{2 t} & \hat{u}_{3 t}\end{array}\right]^{\prime} . \quad \ldots .109$ 
6.7 PPC entre Estados Unidos e Canadá: Resíduos estimados pelo modelo, quantis amostrais e função de autocorrelação parcial (FACP) de $\hat{u}_{t}=\left[\begin{array}{lll}\hat{u}_{1 t} & \hat{u}_{2 t} & \hat{u}_{3 t}\end{array}\right]^{\prime} . \quad$. . . 110

6.8 PPC entre Estados Unidos e Canadá: Série de resíduos de cointegração não normalizados $\hat{e}_{t}(\mathbf{a})$ e normalizados $\hat{e}_{t}^{*}(\mathbf{b}) \ldots \ldots \ldots \ldots$. . . . . . . . . 110

6.9 PPC entre Estados Unidos e Canadá: Componentes $\hat{\beta}_{1 t}$ (a), $\hat{\beta}_{2 t}$ (b) e $\hat{\beta}_{3 t}$ (c) de $\hat{\boldsymbol{\beta}}_{t}=\left[\hat{\beta}_{1 t} \hat{\beta}_{2 t} \hat{\beta}_{3 t}\right]^{\prime}$ juntamente com bandas bootstrap de $95 \%$. Para efeito comparativo, os três componentes são apresentadas no mesmo gráfico (d). . . . . 111

6.10 PPC entre Estados Unidos e Canadá: Componentes $\hat{\beta}_{1 t}$ (a), $\hat{\beta}_{2 t}$ (b) e $\hat{\beta}_{3 t}$ (c) de $\hat{\boldsymbol{\beta}}_{t}=\left[\hat{\beta}_{1 t} \hat{\beta}_{2 t} \hat{\beta}_{3 t}\right]^{\prime}$ juntamente com bandas bootstrap de $95 \%$. Para efeito comparativo, os três componentes são apresentadas no mesmo gráfico (d). . . . . 111

6.11 PPC entre Estados Unidos e Canadá: Histograma, densidade e quantis bootstrap $2,5 \%$ e $97,5 \%$ para $R V_{C V T}^{(J)}$ sob H0 (vetor de cointegração constante) (a) e sob HA (b) . . . . . . . . . . . . . . . . . . . . . . . . . 112

6.12 PPC entre Estados Unidos e Canadá: Histograma, densidade e quantis bootstrap 2,5\% e 97,5\% para as componentes $\hat{\alpha}_{11}^{*}(\mathbf{a}), \hat{\alpha}_{21}^{*}(\mathbf{b})$ e $\hat{\alpha}_{31}^{*}(\mathbf{c})$ de $\hat{\boldsymbol{\alpha}}^{*}=$

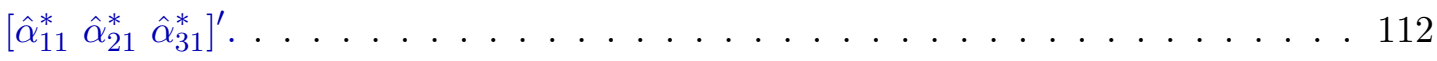

6.13 Gráficos com quantidade $e^{\eta_{t}}$ estimada, juntamente com bandas de confiança, para os países estrangeiros Bélgica, Canadá, Dinamarca, França. . . . . . . . . . . . . 114

6.14 Gráficos com quantidade $e^{\eta_{t}}$ estimada, juntamente com bandas de confiança, para os países estrangeiros Bélgica, Canadá, Dinamarca, França. . . . . . . . . . . . . 115 


\section{Lista de Tabelas}

5.1 Quantis, média e erros padrão empíricos e teóricos para a estatística $R V_{C V T}^{(J)}$, utilizando a ondaleta Haar, obtidos sob $H_{0}$ (cointegração invariante com o tempo). . . . . . . . . . . . . . . . . . 52

5.2 Quantis, média e erros padrão empíricos e teóricos para a estatística $R V_{C V T}^{(J)}$, utilizando a ondaleta Daubechies D2, obtidos sob $H_{0}$ (cointegração invariante com o tempo). . . . . . . . . . . . . . . . . . . 53

5.3 Quantis, média e erros padrão empíricos e teóricos para a estatística $R V_{C V T}^{(J)}$, utilizando a ondaleta Symmlet $S 8$, obtidos sob $H_{0}$ (cointegração invariante com o tempo). . . . . . . . . . . . . . . . . . . 54

5.4 Poder empírico do teste de razão de verossimilhanças $R V_{C V T}^{(J)}$. . . . . . 55

5.5 Ex.01: Estimativa e medidas bootstrap ${ }^{\star}$ sob H0 (vetor de cointegração constante) . . . . . . . . . . . . . . . . . . 59

5.6 Ex.01: Estimativas e medidas bootstrap ${ }^{\star}$ sob HA (inversão de sinal). . . . . 59

5.7 Ex.01: Estatísticas das estimativas dos parâmetros normalizados, obtidas por meio de 1000 processos cointegrados bivariados simulados (inversão de sinal ao longo do tempo). . . . . . . . . . . . . . . . . . . . 62

5.8 Ex.02: Estimativa e medidas bootstrap ${ }^{\star}$ sob H0 (vetor de cointegração constante). . . . . . . . . . . . . . . . . 65

5.9 Ex.02: Estimativas e medidas bootstrap ${ }^{\star}$ sob H0 (vetor de cointegração constante). . . . . . . . . . . . . . . . 6 . 65

5.10 Ex.03: Estimativa e medidas bootstrap ${ }^{\star}$ sob H0 (vetor de cointegração constante). . . . . . . . . . . . . . . . . . 70

5.11 Ex.03: Estimativas e medidas bootstrap ${ }^{\star}$ sob HA (três escalas ao longo do tempo). . . . . . . . . . . . . . . . . 71

5.12 Ex.04: Estimativa e medidas bootstrap ${ }^{\star}$ sob H0 (vetor de cointegração constante). . . . . . . . . . . . . . . . . 75

5.13 Ex.04: Estimativas e medidas bootstrap* sob HA (decrescimento linear) . . 77

5.14 Ex.05: Estimativa e medidas bootstrap ${ }^{\star}$ sob H0 (vetor de cointegração constante) para o modelo completo. . . . . . . . . . . . . . . . 81 
5.15 Ex.05: Estimativas e medidas bootstrap ${ }^{\star}$ sob HA (transição suave entre duas escalas) para o modelo completo. . . . . . . . . . . . . . . . 81

5.16 Modelos candidatos: Exclusão sequencial de parâmetros matriciais $\mathbf{d}_{j k}$. . . 84

5.17 Ex.05: Resultados bootstrap sob H0 (vetor de cointegração constante) para o modelo limiarizado. . . . . . . . . . . . . . . . . . 87

5.18 Ex.05: Estimativas e medidas bootstrap* sob HA (transição suave entre duas escalas) para o modelo limiarizado. . . . . . . . . . . . . . 87

5.19 Ex.06: Estimativa e medidas bootstrap ${ }^{\star}$ sob H0 (vetor de cointegração cons-

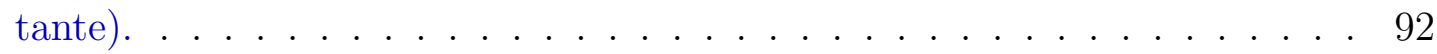

5.20 Ex.06: Estimativas e medidas bootstrap* sob HA (decrescimento linear) . . 95

6.1 Estatística $R V_{C V T}^{(J)}$ e medidas bootstrap $\left(^{\star}\right)$ para testar a PPC constante ao longo do tempo versus PPC variando com o tempo. . . . . . . . . . . . . . 101

6.2 Resultados dos testes de raiz unitária $(H 0)$ e de normalidade $(H 0)$ para os resíduos de cointegração estimados $\hat{e}_{t}=\hat{\boldsymbol{\beta}}_{t}^{\prime} Y_{t} \ldots$. . . . . . . . . . . . . . . 104

6.3 Estimativa e medidas bootstrap $\left(^{\star}\right)$ para a matriz de cargas $\boldsymbol{\alpha}$. . . . . . . . 108

6.4 Estimativa e medidas bootstrap ${ }^{\star}$ sob H0 (vetor de cointegração constante). 113

6.5 Estimativas e medidas bootstrap* sob HA (três escalas ao longo do tempo). 113

6.6 Modelos de PPC: combinação de parâmetros do modelo, valores- $p$ para o teste de normalidade de Shapiro-Wilk para resíduos do modelo $u_{t}=$ $\left(u_{1 t}, u_{2 t}, u_{3 t}\right)^{\prime}$ e estatística de razão de verossimilhanças $R V_{C V T}^{(J)} \ldots \ldots \ldots$

B.1 Estimativas e medidas bootstrap ${ }^{\star}$ sob HA (decrescimento linear para $\beta_{2 t}$ ) - continuação da Tabela 5.13. . . . . . . . . . . . . . . . . . . . . . . . . . 121

B.2 Estimativas e medidas bootstrap ${ }^{\star}$ sob HA (decrescimento logarítmico para $\left.\beta_{2 t}\right)$ - continuação da Tabela 5.20 . . . . . . . . . . . . . . . . . . . 124 


\section{Capítulo 1}

\section{Introdução}

Inúmeros modelos econométricos clássicos são construídos sob a hipótese de que os dados observados tem como origem um processo estacionário, com média e variância constantes ao longo do tempo. Como exemplo, podemos citar os modelos autorregressivos vetoriais de ordem $p$ ou $\operatorname{VAR}(p)$. No entanto, ao inspercionar alguns dados econômicos históricos, é possível perceber que a economia evolui, cresce e muda ao longo do tempo, seja em termos reais ou nominais. Algumas vezes, tais mudanças ocorrem de maneira bastante abrupta (Hendry e Juselius, 2000). Nestes casos, a suposição de estacionariedade não é mais válida, motivando a busca por novos modelos para dados não estacionários.

Conforme aponta Zivot e Wang (2006), as teorias de regressão e de modelos multivariados são apropriadas para modelar conjuntos de dados estacionários, $I(0)$, como os retornos de ativos financeiros e as taxas de crescimento de séries temporais macroeconômicas. A teoria econômica no entanto, sugere a existência de relações de equilíbrio entre os níveis de uma série temporal, que são melhor descritos como sendo processos $I(1)$, isto é, processos não estacionários com diferença estacionária. Além disso, argumentos de arbitragem implicam que os preços de certos ativos financeiros, que são processos $I(1)$, estão conectados. Com base nestas ideias, estaremos interessados no conceito de cointegração para duas ou mais séries temporais. Este conceito é necessário para dar sentido a modelos de regressão e modelos VAR com conjuntos de dados $I(1)$.

Johansen (1995) argumenta que uma teoria econômica é muitas vezes formulada como um conjunto de relações comportamentais ou equações estruturais entre os níveis das variáveis, possivelmente permitindo defasagens. Se as variáveis são $I(1)$, é conveniente reformulá-las em termos de níveis e diferenças, de forma que se uma relação estrutural é modelada por uma relação estacionária, então somos levados a considerar relações estacionárias entre os níveis, isto é, as relações de cointegração.

De modo intuitivo, duas ou mais séries não estacionárias, em particular integradas de ordem $d>0$, denotadas por $I(d)$, são cointegradas se existir uma relação linear de equilíbrio de longo prazo entre elas. Podemos notar a existência destas relações de equilíbrio em variáveis econômicas, como por exemplo, o preço de um mesmo produto 
em diferentes mercados ou taxas de juros com diferentes prazos (Hendry e Juselius, 2000; Morettin, 2011).

Baltagi (2008) destaca que não é uma boa ideia apenas tomar diferenças nas séries temporais não estacionárias $I(1)$, uma vez que este processo acaba por destruir informações potenciais valiosas sobre a relação de longo prazo entre as variáveis econômicas. Tsay (2010) corrobora esta ideia, argumentando que em um sistema cointegrado existem mais componentes não estacionárias (com raiz unitária) do que o número de raízes unitárias. Tomar diferenças individualmente nas séries componentes pode levar à sobre-diferenciação, o que pode trazer problemas na estimação dos parâmetros do modelo. Desta forma, a teoria da cointegração procura estimar esta relação de longo prazo das séries não estacionárias em seus níveis, em vez de trabalhar com suas primeiras diferenças.

Conforme aponta Johansen (1995), o conceito de cointegração é interessante pelo fato desta relação ser capaz de capturar a noção econômica de relação de longo prazo. Por outro lado, cabe ao analista a escolha cuidadosa das variáveis que devem entrar no estudo e discutir com cuidado a teoria econômica que a motiva.

Neste sentido, vamos trabalhar com um modelo difundido na literatura e que leva em consideração as relações de cointegração entre as séries que compõem o modelo. Trabalharemos com o modelo de Paridade de Poder de Compra (PPC). Falk e Wang (2003) apontam que a determinação das taxas de câmbio é de suma importância no estudo das finanças internacionais e em macroeconomia de economias abertas. Além disso, grande parte da literatura moderna sobre a determinação da taxa de câmbio depende da hipótese de PPC. O desenvolvimento da análise de cointegração tem gerado uma série de estudos empíricos, com base na premissa de que a hipótese deve ser vista como uma relação de equilíbrio de longo prazo entre as taxas de câmbio. De acordo com este ponto de vista, se o logaritmo das taxas de câmbio e os níveis de preços nacionais são processos integrados, então eles também devem ser processos cointegrados que se mantêm no longo prazo.

Hayashi (2000) nos informa que podemos utilizar testes de raiz unitária para testar a hipótese de PPC, que segundo o autor, é uma proposição fundamental em estudos de economia internacional, em que as taxas de juros se ajustam aos níveis de preços nacionais.

O presente trabalho tem como foco a análise estatística de séries temporais multivariadas não estacionárias, no âmbito de modelo autorregressivos vetoriais cointegrados (VAR) com erros gaussianos, além de sua reformulação na forma de correção de erros (VEC).

Desde os trabalhos seminais de Engle e Granger (1987) e Johansen (1988), o interesse na literatura sobre o tema cointegração aumentou de maneira expressiva. No entanto, os modelos que tratam deste tema levam em consideração a suposição de que o vetor de cointegração não varia com o tempo. Conforme aponta Bierens e Martins (2010), esta suposição pode ser considerada muito restritiva.

Utilizando os conceitos de cointegração e tendências comuns, o objetivo principal do trabalho será, diferentemente dos modelos de cointegração tradicionais, analisar a hipótese 
econômica de Paridade de Poder de Compra (PPC) variando com o tempo. A abordagem é semelhante à empregada em Bierens e Martins (2010), que modelam o vetor de cointegração temporalmente por meio de polinômios de Chebyshev. Diferentemente de Bierens e Martins (2010), desejamos permitir ao modelo capturar mudanças bruscas nos coeficientes do vetor de cointegração. Com este objetivo, escrevemos o vetor de cointegração na forma de decomposição por ondaletas, que são funções adequadas a este propósito.

O trabalho está dividido da seguinte maneira: No Capítulo 2 apresentamos uma revisão de modelos cointegrados e sua relação com modelos Autorregressivos Vetoriais (VAR), escritos na forma de correção de erros. Além disso, apresentamos o conceito de PPC e sua relação com o conceito de cointegração e com modelos VAR.

No Capítulo 3, apresentamos o conceito de ondaletas. Além disso, apresentamos a representação de um conjunto de dados por meio de decomposição em ondaletas. Descrevemos a seguir as bases de ondaletas Haar, Daublet e Symmlet, empregadas no trabalho.

O Capítulo 4, dedica-se à construção do modelo VAR cointegrado com vetor de cointegração variando com o tempo. São apresentados os passos para a construção da função de verossimilhança condicional e os estimadores de máxima verossimilhança do modelo. O capítulo ainda apresenta a estatística para o teste de razão de verossimilhanças, com o intuito de testar a hipótese de cointegração variando com o tempo. Por fim, o capítulo trata da PPC variando com o tempo para duas situações: considerando e não considerando o intercepto no modelo. Para a segunda, apenas o intercepto varia com o tempo.

O Capítulo 5 dedica-se ao estudo dos quantis empíricos e do poder empírico do teste de razão de verossimilhanças para testar a hipótese de cointegração variando com o tempo. Além disso, apresenta estudos de simulação para avaliar a distribuição dos estimadores e da estatística de razão de verossimilhanças em diferentes casos: cinco modelos sem defasagem e um com defasagem. Para os casos sem defasagem, o primeiro é um exemplo de modelo bivariado simulado que contempla a cointegração variando com o tempo. Para checar a convergência numérica da simulação, apresentamos para o exemplo os resultados de simulações de Monte Carlo para os parâmetros utilizados na simulação. O segundo exemplo por sua vez, contempla um modelo bivariado em que não há cointegração variando com tempo. Do terceiro ao quinto exemplos são consideradas situações mais gerais para o vetor de cointegração variando com o tempo. Por fim, apresentamos no sexto exemplo um caso com defasagem.

O Capítulo 6 dedica-se à apresentação de um conjunto de dados reais utilizados em Falk e Wang (2003) e Bierens e Martins (2010), juntamente com os resultados referentes à aplicação dos modelos apresentados no Capítulo 4 e ao modelo de PPC. Os dados corroboram a hipótese de que os coeficientes de cointegração variam ao longo do tempo, tal qual observado por Bierens e Martins (2010).

O Capítulo 7 dedica-se às considerações finais, onde apresentamos alguns comentários sobre os resultados obtidos, juntamente com direcionamentos para futuras pesquisas. 


\section{Capítulo 2}

\section{Processos cointegrados e Modelo de Correção de Erros Vetorial (VEC)}

Neste capítulo, apresentamos uma revisão de modelos cointegrados e sua relação com modelos Autorregressivos Vetoriais (VAR), escritos na forma de correção de erros. Apresentamos a função de verossimilhança condicional do modelo e os estimadores de máxima verossimilhança. Além disso, apresentamos o conceito de Paridade de Poder de Compra (PPC) e sua relação com o conceito de cointegração. Seguimos de perto os conceitos e definições enunciados em Hendry e Juselius (2001), Falk e Wang (2003), Lutkepohl (2005), Zivot e Wang (2006), Morettin (2011) e Tsay (2013).

\subsection{Processos cointegrados}

Estamos interessados em estabelecer modelos para uma série temporal vetorial $Y_{t}$ nvariada, ou seja, com $n$ componentes $Y_{1 t}, Y_{2 t}, \ldots, Y_{n t}$, observadas em $t=0, \pm 1, \pm 2, \ldots$. Utilizamos a notação $Y_{t}=\left(Y_{1 t}, Y_{2 t}, \ldots, Y_{n t}\right)^{\prime}$, com $t \in \mathbb{Z}$ e $Y_{i t}$ ou $Y_{i, t}$ indistintamente para a $i$-ésima componente de $Y_{t}, \operatorname{com} i=1, \ldots, n$.

Definição 2.1. O vetor de médias $\boldsymbol{\mu}_{t}$ e a matriz de covariâncias $\gamma(t+\tau, t)$ descrevem as propriedades de segunda ordem das séries $Y_{1 t}, Y_{2 t}, \ldots, Y_{n t}$. Estas medidas, em geral, dependem do tempo e são definidas como:

$$
\boldsymbol{\mu}_{t}=E\left(Y_{t}\right)=\left(\mu_{1 t}, \mu_{2 t}, \ldots, \mu_{n t}\right)^{\prime}
$$

$e$

$$
\gamma(t+\tau, t)=E\left\{\left(Y_{t+\tau}-\boldsymbol{\mu}_{t+\tau}\right)\left(Y_{t}-\boldsymbol{\mu}_{t}\right)^{\prime}\right\},
$$

que é uma matriz de ordem $n \times n$. 
Na diagonal principal de $\gamma(t+\tau, t)$ temos as autocovariâncias das séries individuais, calculadas nos instantes $t+\tau$ e $t$, enquanto fora da diagonal principal estão as covariâncias cruzadas entre as séries $Y_{i, t+\tau}$ e $Y_{j, t}$, para $i \neq j$.

Denotando por $\gamma_{i, j}(t+\tau, t)$ as componentes da matriz $\gamma(t+\tau, t)$, temos que

$$
\gamma_{i, j}(t+\tau, t)=\operatorname{Cov}\left\{Y_{i, t+\tau} Y_{j, t}\right\}:=E\left\{\left(Y_{i, t+\tau}-\mu_{i, t+\tau}\right)\left(Y_{j, t}-\mu_{j, t}\right)\right\}
$$

é a covariância entre as variáveis $Y_{i, t+\tau}$ e $Y_{j, t}$, para $i, j=1, \ldots, n$.

Estamos interessados, no entanto, no caso em que tanto o vetor de médias $\boldsymbol{\mu}_{t}$ quanto a matriz de covariâncias $\gamma(t+\tau, t)$ não dependem de $t$. Neste caso, estamos trabalhando com séries estacionárias, ou fracamente estacionárias, cuja definição é dada a seguir:

Definição 2.2. Uma série temporal $n$-variada $Y_{t}$ é dita fracamente estacionária se ela possui média $\boldsymbol{\mu}_{t}$ e matriz de covariâncias $\gamma(t+\tau, t)$ que não dependem do instante de tempo $t$, para $t, \tau \in \mathbb{Z}$. Nesta situação, temos que

$$
\boldsymbol{\mu}=E\left(Y_{t}\right)=\left(\mu_{1}, \mu_{2}, \ldots, \mu_{n}\right)^{\prime}
$$

e

$$
\gamma(\tau)=E\left\{\left(Y_{t+\tau}-\boldsymbol{\mu}\right)\left(Y_{t}-\boldsymbol{\mu}\right)^{\prime}\right\}=\left\{\gamma_{i, j}(\tau)\right\}_{(i, j)=1}^{n},
$$

$\forall \tau \in \mathbb{Z}$. Neste caso, $\gamma_{i i}(\tau)$ será a função de autocovariância da série estacionária $Y_{i t} e$ $\gamma_{i j}(\tau)$ será a função de covariância cruzada de $Y_{i t}$ e $Y_{j t}$.

Da Definição 2.2, podemos notar que em geral, $\gamma_{i j}(\tau) \neq \gamma_{j i}(\tau)$. Além disso, para o caso particular em que $\tau=0$ na Equação 2.5, temos que

$$
\gamma(0)=E\left\{\left(Y_{t}-\boldsymbol{\mu}\right)\left(Y_{t}-\boldsymbol{\mu}\right)^{\prime}\right\}
$$

que é a matriz de covariâncias contemporâneas. Notamos ainda que $\gamma_{i, i}(0)=\operatorname{Var}\left(X_{i t}\right)$ e que $\gamma_{i, j}(0)=\operatorname{Cov}\left(X_{i t}, X_{j t}\right)$.

Definição 2.3. Seja $\Delta^{d}$ o operador diferença usual de séries temporais. Um processo $Y_{t}$ é integrado de ordem d se $\Delta^{d} Y_{t}$ for estacionário, e neste caso, utilizamos a seguinte notação: $Y_{t} \sim I(d)$. Em particular um processo estacionário é $I(0)$.

Se $Y_{1 t}$ e $Y_{2 t}$ forem processos $I(d)$, então a combinação linear $Y_{t}=Y_{1 t}-\beta Y_{2 t}$ será, em geral, também $I(d)$. Mas é possível que $Y_{t}$ seja integrado de ordem menor, digamos $I(d-b)$, com $0<b \leq d$. Se tivermos uma situação em que $d=b=1$, temos que $Y_{1 t}$ e $Y_{2 t}$ são $I(1)$ e consequentemente, $Y_{t}$ será $I(0)$. Neste caso, diremos que $Y_{1 t}$ e $Y_{2 t}$ são cointegrados.

Seja $Y_{t}$ um vetor $(n \times 1)$. Diremos que $Y_{t}$ é integrado de ordem $d, I(d)$, se $d$ for a maior ordem de integração das séries individuais. Em outras palavras, se $Y_{t}=\left(Y_{1 t}, \ldots, Y_{n t}\right)^{\prime}$, com 
$Y_{i t} \sim I\left(d_{i}\right)$, então $d=\max \left(d_{1}, \ldots, d_{n}\right)$. Podemos resumir estas afirmações na Definição 2.4 a seguir:

Definição 2.4. As componentes do vetor $Y_{t}$ são cointegradas de ordem $(d, b)$ se todas as componentes de $Y_{t}$ são $I(d)$ e se existe um vetor $\boldsymbol{\beta}=\left(\beta_{1}, \ldots, \beta_{n}\right)^{\prime}$, de ordem $(n \times 1)$ não nulo, tal que

$$
\boldsymbol{\beta}^{\prime} Y_{t}=\beta_{1} Y_{1 t}+\beta_{2} Y_{2 t}+\ldots+\beta_{n} Y_{n t} \sim I(d-b),
$$

com $0<b \leq d$. O vetor $\boldsymbol{\beta}$ de ordem $(n \times 1)$ é chamado de vetor cointegrado ou vetor de cointegração.

A teoria usual de mínimos quadrados ordinários também se aplica a séries $I(0)$. Neste caso, os resultados estatísticos para a regressão linear também são válidos. Se uma ou todas as variáveis na regressão são $I(1)$, então os resultados estatísticos podem não valer. Um caso importante em que os resultados estatísticos não valem é o da regressão espúria, onde todas as séries são $I(1)$ e não cointegradas. Logo, fazer uma regressão linear com séries $I(1)$ só faz sentido se estas forem cointegradas (Zivot e Wang, 2006).

$\mathrm{O}$ vetor de cointegração $\boldsymbol{\beta}$ não é único, pois se para $c \neq 0$, o novo vetor $\boldsymbol{\beta}^{*}=c \boldsymbol{\beta}$ também será um vetor de cointegração. Consequentemente, a combinação linear $c \boldsymbol{\beta}^{\prime} Y_{t}=$ $\boldsymbol{\beta}^{* \prime} Y_{t}$ também será $I(0)$. Desta forma, alguma normalização é requerida para identificar $\boldsymbol{\beta}$ unicamente.

Se alguns elementos de $\boldsymbol{\beta}$ são iguais a zero, então somente o subconjunto das séries em $Y_{t}$ cujos coeficiente são não nulos é cointegrado. A combinação $\boldsymbol{\beta}^{\prime} Y_{t}$ é geralmente motivada pela teoria econômica e se refere às relações de equilíbrio de longo prazo. Assume-se que séries $I(1)$ com relações de equilíbrio de longo prazo não podem se distanciar muito do equilíbrio, uma vez que as forças econômicas irão agir para restaurar a relação de equilíbrio (Zivot e Wang, 2006).

Uma das variáveis é geralmente utilizada para normalizar $\boldsymbol{\beta}$, fixando-se seu coeficiente igual a um. Usualmente, toma-se $\boldsymbol{\beta}=\left(1,-\beta_{2}, \ldots,-\beta_{n}\right)^{\prime}$, de modo que

$$
\boldsymbol{\beta}^{\prime} Y_{t}=Y_{1 t}-\beta_{2} Y_{2 t}-\ldots-\beta_{n} Y_{n t}
$$

Se por exemplo, $\boldsymbol{\beta}^{\prime} Y_{t} \sim I(0)$, temos que

$$
Y_{1 t}=\beta_{2} Y_{2 t}+\ldots+\beta_{n} Y_{n t}+u_{t}
$$

com $u_{t} \sim I(0)$. Dizemos que $u_{t}$ é o resíduo de cointegração ou erro de desequilíbrio. Em equilíbrio de longo prazo temos que $u_{t}=0$ e a relação de equilíbrio de longo prazo é

$$
Y_{1 t}=\beta_{2} Y_{2 t}+\ldots+\beta_{n} Y_{n t}
$$


Se $Y_{t}$ possuir $n>2$ componentes, podem existir infinitos vetores de cointegração. Se existirem exatamente $r$ vetores de cointegração linearmente independentes, com $0<r<$ $n$, então eles podem ser reunidos na matriz de cointegração $\boldsymbol{\beta}$ de ordem $(n \times r)$ de posto $r$, chamado de posto de cointegração. Neste caso, a matriz

$$
\boldsymbol{\beta}^{\prime} Y_{t}=\left[\begin{array}{c}
\boldsymbol{\beta}_{1}^{\prime} Y_{t} \\
\vdots \\
\boldsymbol{\beta}_{r}^{\prime} Y_{t}
\end{array}\right]=\left[\begin{array}{c}
u_{1 t} \\
\vdots \\
u_{r t}
\end{array}\right]
$$

é estacionária, ou seja, $I(0)$. Note ainda que

$$
\boldsymbol{\beta}^{\prime}=\left[\begin{array}{c}
\boldsymbol{\beta}_{1}^{\prime} \\
\vdots \\
\boldsymbol{\beta}_{r}^{\prime}
\end{array}\right]_{r \times n}
$$

forma uma base para o espaço de vetores de cointegração. Além disso, os vetores linearmente independentes $\boldsymbol{\beta}_{\mathbf{1}}, \ldots, \boldsymbol{\beta}_{\boldsymbol{r}}$ na base de cointegração $\boldsymbol{\beta}$ não é única, a menos que alguma suposição de normalização seja feita. Isto é, qualquer combinação linear de $\boldsymbol{\beta}_{1}, \ldots, \boldsymbol{\beta}_{r}$, por exemplo, $\boldsymbol{\beta}_{s}=c_{1} \boldsymbol{\beta}_{1}+\ldots+c_{r} \boldsymbol{\beta}_{r}$ também será um vetor de cointegração.

Séries cointegradas apresentam uma tendência estocástica comum. Isto quer dizer que séries cointegradas caminham juntas no longo prazo, dado que uma combinação linear delas é reversível à média (estacionária). De modo geral, se o vetor $Y_{t}$ for cointegrado com $r$ vetores de cointegração e $0<r<n$, existirão então $n-r$ tendências estocásticas comuns.

\subsection{Modelo VAR na forma de correção de erros}

Nesta seção, apresentamos os conceitos e definições apresentados em Lutkepohl (2005), Zivot e Wang (2006) e Morettin (2011).

O modelo autorregressivo vetorial de ordem $p, \operatorname{VAR}(p)$, é um dos mais bem sucedidos e flexíveis para séries temporais multivariadas estacionárias, tendo se mostrado útil para descrever o comportamento dinâmico destas séries.

Sejam $n$ séries temporais alocadas em um vetor aleatório $Y_{t}=\left(Y_{1 t}, Y_{2 t}, \ldots, Y_{n t}\right)^{\prime}$ de ordem $(n \times 1)$. Um modelo autorregressivo vetorial $\operatorname{VAR}(p) n$-dimensional para $Y_{t}$ é dado por

$$
Y_{t}=\boldsymbol{\nu}+\boldsymbol{\Phi}_{1} Y_{t-1}+\ldots+\boldsymbol{\Phi}_{p} Y_{t-p}+u_{t}
$$

onde $\boldsymbol{\Phi}_{s}$ são matrizes de coeficientes $(n \times n)$ fixas com elementos $\phi_{i j, s}, \operatorname{com} i, j=1, \ldots, n$ 
e $s=1, \ldots, p$. Por sua vez, $\boldsymbol{\nu}=\left(\nu_{1}, \ldots, \nu_{n}\right)^{\prime}$ é um vetor $(n \times 1)$ fixo de interceptos, permitindo que $E\left(Y_{t}\right)$ seja diferente de zero. Além disso, $u_{t}=\left(u_{1 t}, \ldots, u_{n t}\right)^{\prime}$ é um ruído branco ou processo de inovaçôes $n$-dimensional, ou seja, $E\left(u_{t}\right)=\mathbf{0}, E\left(u_{t} u_{t}^{\prime}\right)=\boldsymbol{\Sigma}_{u} \mathrm{e}$ $E\left(u_{t} u_{s}^{\prime}\right)=\mathbf{0}$ para $s \neq t$.

Proposição 2.1. Para o modelo VAR(p) dado na Equação 2.8, temos os seguintes resultados:

O processo $Y_{t}$ será estacionário se as soluções de

$$
\left|\boldsymbol{I}_{n}-\boldsymbol{\Phi}_{1} z-\ldots-\boldsymbol{\Phi}_{p} z^{p}\right|=0
$$

estiverem fora do círculo unitário. Além disso, se $Y_{t}$ for estacionário, então

$$
\boldsymbol{\mu}=E\left(Y_{t}\right)=\left(\boldsymbol{I}_{n}-\boldsymbol{\Phi}_{1} z-\ldots-\boldsymbol{\Phi}_{p} z^{p}\right)^{-1} \boldsymbol{\nu}
$$

Demonstração: Veja Morettin (2011).

Podemos ver que a cointegração pode ocorrer em um processo $\operatorname{VAR}(p) n$-dimensional, como o dado na Equação 2.8. Suponha que o processo $Y_{t}$ seja não estacionário, com uma ou mais soluções da Equação 2.9 sobre o círculo unitário. Como $\left|\boldsymbol{I}_{n}-\boldsymbol{\Phi}_{1}-\ldots-\boldsymbol{\Phi}_{p}\right|=0$, a matriz $\boldsymbol{\Pi}=-\left(\boldsymbol{I}_{n}-\boldsymbol{\Phi}_{1}-\ldots-\boldsymbol{\Phi}_{p}\right)$ é singular. Suponha que $\boldsymbol{\Pi}$ possua posto $\rho(\boldsymbol{\Pi})=$ $r<n$. Desta forma, $\Pi$ pode ser decomposta como

$$
\Pi=\boldsymbol{\alpha} \boldsymbol{\beta}^{\prime},
$$

onde $\boldsymbol{\alpha}$ e $\boldsymbol{\beta}$ são matrizes de ordem $n \times r$.

Vamos supor ainda que todas as componentes de $Y_{t}$ sejam $I(1)$, sendo desta forma estacionárias sob primeira diferença. Assim, podemos reescrever a Equação 2.8 na forma de correção de erros, aqui chamado de modelo VEC, como

$$
\begin{aligned}
\Delta Y_{t} & =\boldsymbol{\nu}+\Pi Y_{t-1}+\boldsymbol{\Gamma}_{1} \Delta Y_{t-1}+\ldots+\boldsymbol{\Gamma}_{p-1} \Delta Y_{t-p+1}+u_{t}, \\
& =\boldsymbol{\nu}+\boldsymbol{\alpha} \boldsymbol{\beta}^{\prime} Y_{t-1}+\boldsymbol{\Gamma}_{1} \Delta Y_{t-1}+\ldots+\boldsymbol{\Gamma}_{p-1} \Delta Y_{t-p+1}+u_{t},
\end{aligned}
$$

onde $\boldsymbol{\Gamma}_{i}=-\left(\boldsymbol{\Phi}_{i+1}+\ldots+\boldsymbol{\Phi}_{p}\right)$ são matrizes $(n \times n)$, para $i=1, \ldots, p-1$ e $\Delta Y_{t}=Y_{t}-Y_{t-1}$.

A forma como o modelo VAR está escrito na Equação 2.12 é chamada de forma de correção de equilíbrio ou forma de correção de erros. Podemos ainda reescrever esta equação como

$$
\boldsymbol{\alpha} \boldsymbol{\beta}^{\prime} Y_{t-1}=\Delta Y_{t}-\boldsymbol{\nu}-\boldsymbol{\Gamma}_{1} \Delta Y_{t-1}-\ldots-\boldsymbol{\Gamma}_{p-1} \Delta Y_{t-p+1}-u_{t} .
$$

Note que o segundo termo de 2.13 é $I(0)$. Logo, $\boldsymbol{\alpha} \boldsymbol{\beta}^{\prime} Y_{t-1}$ também será $I(0)$. Além disso, é possível obter um processo $I(0)$ ao multiplicar $\left[\left(\boldsymbol{\alpha}^{\prime} \boldsymbol{\alpha}\right)^{-1} \boldsymbol{\alpha}^{\prime}\right]$ por $\boldsymbol{\alpha} \boldsymbol{\beta}^{\prime} Y_{t-1}$. Desta 
forma, temos que

$$
\left[\left(\boldsymbol{\alpha}^{\prime} \boldsymbol{\alpha}\right)^{-1} \boldsymbol{\alpha}^{\prime}\right] \boldsymbol{\alpha} \boldsymbol{\beta}^{\prime} Y_{t-1}=\boldsymbol{\beta}^{\prime} Y_{t-1} \sim I(0) .
$$

Segue então que cada linha de $\boldsymbol{\beta}^{\prime} Y_{t-1}$ representará uma relação de cointegração. Neste caso, diremos que o sistema é cointegrado de posto $r$, com matriz de cointegração $\boldsymbol{\beta}$ e matriz de cargas $\boldsymbol{\alpha}$.

Se a representação de um processo cointegrado é dada na Equação 2.12, é fácil recuperar a representação VAR na forma da Equação 2.8, utilizando as expressões

$$
\left\{\begin{array}{l}
\boldsymbol{\Phi}_{1}=\boldsymbol{\Pi}+\boldsymbol{I}_{k}+\boldsymbol{\Gamma}_{1} \\
\boldsymbol{\Phi}_{i}=\boldsymbol{\Gamma}_{i}-\boldsymbol{\Gamma}_{i-1}, i=2, \ldots, p-1 \\
\boldsymbol{\Phi}_{p}=-\boldsymbol{\Gamma}_{p-1} .
\end{array}\right.
$$

\subsection{Função de verossimilhança condicional}

Vamos considerar inicialmente o modelo dado na Equação 2.12 sem o vetor de termos determinísticos

$$
\Delta Y_{t}=\Pi Y_{t-1}+\Gamma_{1} \Delta Y_{t-1}+\ldots+\boldsymbol{\Gamma}_{p-1} \Delta Y_{t-p+1}+u_{t}
$$

onde $Y_{t}$ é um processo de dimensão $n$, onde cada variável individual $Y_{i t}$ é $I(1)$, para $i=1, \ldots, n$, embora $r$ combinações lineares de $Y_{t}$ sejam estacionárias. Conforme visto anteriormente, esta suposição nos permite escrever $\Pi=\boldsymbol{\alpha} \boldsymbol{\beta}^{\prime}, \forall t=1, \ldots, T$, sendo $\rho(\Pi)=$ $r$ e $0<r<n, \boldsymbol{\alpha}$ e $\boldsymbol{\beta}$ matrizes $(n \times r)$, com posto $\rho(\boldsymbol{\alpha})=\rho(\boldsymbol{\beta})=r$ e $T$ sendo o número de observações da amostra $n$ variada. Vamos supor ainda que na Equação 2.12, $u_{t}$ são i.i.d. e seguem uma distribuição $N_{n}\left(\mathbf{0}, \boldsymbol{\Sigma}_{u}\right)$.

Reescrevendo o modelo VEC dado na Equação 2.15 em notação matricial, temos

$$
\Delta Y=\Pi Y_{-1}+\Gamma \Delta X+U
$$

onde

$$
\begin{aligned}
\Delta Y & :=\left[\begin{array}{llll}
\Delta Y_{1} & \Delta Y_{2} & \ldots & \Delta Y_{T}
\end{array}\right] \\
Y_{-1} & :=\left[\begin{array}{llll}
Y_{0} & Y_{1} & \ldots & Y_{T-1}
\end{array}\right], \\
\Gamma & :=\left[\begin{array}{lll}
\Gamma_{1} & \ldots & \boldsymbol{\Gamma}_{p-1}
\end{array}\right]
\end{aligned}
$$




$$
\begin{gathered}
\Delta X=\left[\begin{array}{llll}
\Delta X_{0} & \Delta X_{1} & \ldots & \Delta X_{T-1}
\end{array}\right], \operatorname{com} \quad \Delta X_{t-1}=\left[\begin{array}{c}
\Delta Y_{t-1} \\
\vdots \\
\Delta Y_{t-p+1}
\end{array}\right] \\
U=\left[\begin{array}{lll}
U_{1} & \ldots & U_{T}
\end{array}\right]
\end{gathered}
$$

Considere uma amostra de $(T+p)$ observações para $\Delta Y=\left(\Delta Y_{1 t}, \ldots, \Delta Y_{n t}\right)^{\prime}$, para $t=1-p, \ldots, 0,1, \ldots, T$, denotada por

$$
(\underbrace{\Delta Y_{1-p}, \Delta Y_{2-p}, \ldots, \Delta Y_{0}}_{p \text { obs. }}, \underbrace{\Delta Y_{1}, \ldots, \Delta Y_{T}}_{T \text { obs. }}) .
$$

Vamos basear nossa estimação nas últimas $T$ observações das séries de $\Delta Y_{t}$, condicionando nas primeiras $p$ observações. O objetivo será construir a verossimilhança condicional

$$
l(\boldsymbol{\theta})=f\left(\Delta Y_{T}, \Delta Y_{T-1}, \ldots, \Delta Y_{1} \mid \Delta Y_{0}, \ldots, \Delta Y_{1-p} ; \boldsymbol{\theta}\right),
$$

e maximizar com respeito a $\boldsymbol{\theta}=\left(\boldsymbol{\Pi}, \boldsymbol{\Gamma}_{1}, \ldots, \boldsymbol{\Gamma}_{p-1}, \boldsymbol{\Sigma}_{u}\right)$.

Condicional às observações até o instante $t-1, \Delta Y_{t}$ é igual a um termo constante acrescido de um termo com distribuição normal, ou seja

$$
\Delta Y_{t} \mid \Delta Y_{t-1}, \ldots, \Delta Y_{1-p}=\underbrace{\boldsymbol{\alpha} \boldsymbol{\beta}^{\prime} Y_{t-1}+\boldsymbol{\Gamma} \Delta X_{t-1}}_{\text {constante }}+u_{t}
$$

$\operatorname{com} u_{t} \sim \mathbf{N}_{n}\left(\mathbf{0}, \boldsymbol{\Sigma}_{u}\right)$.

Pela Equação 2.15, temos ainda que $E\left(\Delta Y_{t} \mid \Delta Y_{t-1}, \ldots, \Delta Y_{1-p}\right)=\boldsymbol{\alpha} \boldsymbol{\beta}^{\prime} Y_{t-1}+\boldsymbol{\Gamma} \Delta X_{t-1}$, enquanto $\operatorname{Var}\left(\Delta Y_{t} \mid \Delta Y_{t-1}, \ldots, \Delta Y_{1-p}\right)=\Sigma_{u}$. Podemos então concluir que

$$
\Delta Y_{t} \mid \Delta Y_{t-1}, \ldots, \Delta Y_{1-p} \sim \mathbf{N}_{n}\left(\boldsymbol{\alpha} \boldsymbol{\beta}^{\prime} Y_{t-1}+\boldsymbol{\Gamma} \Delta X_{t-1}, \boldsymbol{\Sigma}_{u}\right)
$$

A densidade da t-ésima observação, será dada então por

$$
\begin{aligned}
f\left(\Delta Y_{t} \mid \Delta Y_{t-1}, \ldots, \Delta Y_{1-p} ; \boldsymbol{\theta}\right)= & \frac{1}{(2 \pi)^{\frac{n}{2}}\left|\boldsymbol{\Sigma}_{u}\right|^{\frac{1}{2}}} \cdot \exp \left(-\frac{1}{2}\left(\Delta Y_{t}-\boldsymbol{\alpha} \boldsymbol{\beta}^{\prime} Y_{t-1}-\boldsymbol{\Gamma} \Delta X_{t-1}\right)^{\prime} .\right. \\
& \left.\cdot \boldsymbol{\Sigma}_{u}^{-1} \cdot\left(\Delta Y_{t}-\boldsymbol{\alpha} \boldsymbol{\beta}^{\prime} Y_{t-1}-\boldsymbol{\Gamma} \Delta X_{t-1}\right)\right)
\end{aligned}
$$

Logo, a verossimilhança condicional para a amostra completa $\Delta Y_{T}, \Delta Y_{T-1}, \ldots, \Delta Y_{1} \mid$ 
$\Delta Y_{0}, \ldots, \Delta Y_{1-p}$ é dada por

$$
f\left(\Delta Y_{T}, \Delta Y_{T-1}, \ldots, \Delta Y_{1} \mid \Delta Y_{0}, \ldots, \Delta Y_{1-p} ; \boldsymbol{\theta}\right)=\prod_{t=1}^{T} f\left(\Delta Y_{t} \mid \Delta Y_{t-1}, \ldots, \Delta Y_{1-p} ; \boldsymbol{\theta}\right) .
$$

Estamos interessados no entanto na função de log-verossimilhança $\mathscr{L}(\boldsymbol{\theta})$ para o modelo dado na Equação 2.16. Substituindo a Equação 2.25 em 2.26, mostramos no Apêndice B.1, página 120, que a log-verossimilhança, em notação matricial, é dada por

$$
\begin{aligned}
& \mathscr{L}(\boldsymbol{\theta})=-\frac{n T}{2} \ln (2 \pi)-\frac{T}{2} \ln \left(\left|\boldsymbol{\Sigma}_{u}\right|\right)-\frac{1}{2} \operatorname{tr}\left(\left(\Delta Y-\boldsymbol{\alpha} \boldsymbol{\beta}^{\prime} Y_{-1}-\boldsymbol{\Gamma} \Delta X\right)^{\prime} \cdot \boldsymbol{\Sigma}_{u}^{-1} .\right. \\
&\left.\cdot\left[\Delta Y-\boldsymbol{\alpha} \boldsymbol{\beta}^{\prime} Y_{-1}-\boldsymbol{\Gamma} \Delta X\right]\right) .
\end{aligned}
$$

Nosso objetivo então será escolher $\boldsymbol{\theta}=\left(\boldsymbol{\Pi}, \boldsymbol{\Gamma}_{1}, \ldots, \boldsymbol{\Gamma}_{p-1}, \boldsymbol{\Sigma}_{u}\right)$ que maximiza 2.27 sujeito à restrição de que $\boldsymbol{\Pi}=\boldsymbol{\alpha} \boldsymbol{\beta}^{\prime}$.

\subsection{Estimadores de máxima verossimilhança}

Uma vez obtida a função de verossimilhança para o caso gaussiano, estamos interessados em obter os estimadores de máxima verossimilhança para o modelo VEC dado na Equação 2.16. Vamos concentrar a função de verossimilhança com respeito ao termo de correção de erros, substituindo a matriz de parâmetros $\Gamma$ (de curto prazo) pelo seu estimador de mínimos quadrados, dada uma matriz $\Pi$ fixa (Lutkepohl, 2005):

$$
\hat{\boldsymbol{\Gamma}}(\boldsymbol{\Pi})=\left(\Delta Y-\Pi Y_{-1}\right) \Delta X^{\prime}\left(\Delta X \Delta X^{\prime}\right)^{-1} .
$$

Substituindo $\hat{\boldsymbol{\Gamma}}(\boldsymbol{\Pi})$ por $\boldsymbol{\Gamma}$ na Equação 2.16, temos que

$$
\begin{aligned}
& \Delta Y=\Pi Y_{-1}+\hat{\boldsymbol{\Gamma}}(\boldsymbol{\Pi}) \Delta X+U^{*} \\
& \Delta Y=\Pi Y_{-1}+\left(\Delta Y-\Pi Y_{-1}\right) \Delta X^{\prime}\left(\Delta X \Delta X^{\prime}\right)^{-1} \Delta X+U^{*} \\
& \Delta Y=\Pi Y_{-1}+\Delta Y \Delta X^{\prime}\left(\Delta X \Delta X^{\prime}\right)^{-1} \Delta X-\Pi Y_{-1} \Delta X^{\prime}\left(\Delta X \Delta X^{\prime}\right)^{-1} \Delta X+U^{*} \\
& \Delta Y=\Pi Y_{-1} \underbrace{\left[\mathbf{I}_{T}-\Delta X^{\prime}\left(\Delta X \Delta X^{\prime}\right)^{-1} \Delta X\right]}_{M}+\Delta Y \Delta X^{\prime}\left(\Delta X \Delta X^{\prime}\right)^{-1} \Delta X+U^{*} \\
& \Delta Y=\Pi Y_{-1} \mathbf{M}+\Delta Y \Delta X^{\prime}\left(\Delta X \Delta X^{\prime}\right)^{-1} \Delta X+U^{*} .
\end{aligned}
$$

Rearranjando os termos da igualdade em 2.29, temos que 


$$
\begin{aligned}
\Delta Y \underbrace{\left[\mathbf{I}_{T}-\Delta X^{\prime}\left(\Delta X \Delta X^{\prime}\right)^{-1} \Delta X\right]}_{\mathbf{M}} & =\Pi Y_{-1} \mathbf{M}+U^{*} \\
\underbrace{\Delta Y \mathbf{M}}_{R_{0}} & =\Pi \underbrace{Y_{-1} \mathbf{M}}_{R_{1}}+U^{*} \\
R_{0} & =\Pi R_{1}+U^{*},
\end{aligned}
$$

$\operatorname{com} \mathbf{M}=\left[\mathbf{I}_{T}-\Delta X^{\prime}\left(\Delta X \Delta X^{\prime}\right)^{-1} \Delta X\right], R_{0}=\Delta Y \mathbf{M}$ e $R_{1}=Y_{-1} \mathbf{M}$.

Conforme aponta Lutkepohl (2005), devemos notar que $R_{0}$ é o resíduo de uma regressão multivariada de $\Delta Y$ em $\Delta X$. Por outro lado, $R_{1}$ é o resíduo de uma regressão multivariada de $Y_{-1}$ em $\Delta X$.

Enunciamos a seguir a Proposição 2.2 (Johansen, 1988; Lutkepohl, 2005) que nos dá os estimadores de máxima verossimilhança para os parâmetros do modelo VEC.

Proposição 2.2. (Estimadores de máxima verossimilhança de um modelo $V E C$ )

Sejam as matrizes $\mathbf{M}:=\left[\mathbf{I}_{T}-\Delta X^{\prime}\left(\Delta X \Delta X^{\prime}\right)^{-1} \Delta X\right], R_{0}:=\Delta Y \mathbf{M}$ e $R_{1}:=Y_{-1} \mathbf{M}$, apresentadas em 2.30. Definimos as matrizes de covariância amostrais:

$$
\mathbf{S}_{i, j}:=\frac{1}{T} R_{i} R_{j}^{\prime}, \text { com } i, j=0,1 .
$$

Em seguida, ao resolver o problema de autovalores

$$
\left|\lambda \mathbf{S}_{11}-\mathbf{S}_{10} \mathbf{S}_{00}^{-1} \mathbf{S}_{01}\right|=0,
$$

obtemos os autovalores ordenados $\hat{\lambda}_{1} \geq \hat{\lambda}_{2} \geq \ldots \geq \hat{\lambda}_{n}$ e autovetores associados $\hat{\boldsymbol{v}}_{1}, \hat{\boldsymbol{v}}_{2}, \ldots, \hat{\boldsymbol{v}}_{n}$, que satisfazem

$$
\begin{aligned}
& \hat{\lambda}_{i} \mathbf{S}_{11} \hat{\boldsymbol{v}}_{i}=\mathbf{S}_{10} \mathbf{S}_{00}^{-1} \mathbf{S}_{01} \\
& \hat{\mathbf{V}} \mathbf{S}_{11} \hat{\mathbf{V}}=\mathbf{I}_{n}
\end{aligned}
$$

$\operatorname{com} \hat{\mathbf{V}}=\left[\hat{\boldsymbol{v}}_{1}, \hat{\boldsymbol{v}}_{2}, \ldots, \hat{\boldsymbol{v}}_{n}\right]$. Desta forma, a log-verossimilhança do modelo VEC dado na Equação 2.16 é maximizada para

$$
\begin{gathered}
\hat{\boldsymbol{\beta}}^{\prime}=\left[\hat{\boldsymbol{v}}_{1}, \ldots, \hat{\boldsymbol{v}}_{r}\right] \mathbf{S}_{11}^{-\frac{1}{2}}, \\
\hat{\boldsymbol{\alpha}}=\Delta Y \mathbf{M} Y_{-1}^{\prime} \hat{\boldsymbol{\beta}}\left(\hat{\boldsymbol{\beta}}^{\prime} Y_{-1} \mathbf{M} Y_{-1}^{\prime} \hat{\boldsymbol{\beta}}\right)^{-1}=\mathbf{S}_{01} \hat{\boldsymbol{\beta}}\left(\hat{\boldsymbol{\beta}}^{\prime} \mathbf{S}_{11} \hat{\boldsymbol{\beta}}^{\prime}\right)^{-1} \\
\hat{\boldsymbol{\Gamma}}=\left(\Delta Y-\hat{\boldsymbol{\alpha}} \hat{\boldsymbol{\beta}}^{\prime} Y_{-1}\right) \Delta X^{\prime}\left(\Delta X \Delta X^{\prime}\right)^{-1}
\end{gathered}
$$




$$
\hat{\boldsymbol{\Sigma}}_{u}=\frac{1}{T}\left(\Delta Y-\hat{\boldsymbol{\alpha}} \hat{\boldsymbol{\beta}}^{\prime} Y_{-1}-\hat{\boldsymbol{\Gamma}} \Delta X\right)\left(\Delta Y-\hat{\boldsymbol{\alpha}} \hat{\boldsymbol{\beta}}^{\prime} Y_{-1}-\hat{\boldsymbol{\Gamma}} \Delta X\right)^{\prime} .
$$

Além disso, o máximo de 2.16 é atingido em

$$
\max \mathscr{L}(\boldsymbol{\theta})=-\frac{n T}{2} \ln 2 \pi-\frac{T}{2}\left[\ln \left|\mathbf{S}_{00}\right|+\sum_{i=1}^{r} \ln \left(1-\lambda_{i}\right)\right]-\frac{n T}{2} .
$$

\section{Demonstração:}

Para $\boldsymbol{\alpha}$ e $\boldsymbol{\beta}$ fixos (П fixo), o máximo de $\mathscr{L}(\boldsymbol{\theta})$ em 2.27 é atingido para

$$
\hat{\Gamma}\left(\boldsymbol{\alpha} \boldsymbol{\beta}^{\prime}\right)=\left(\Delta Y-\boldsymbol{\alpha} \boldsymbol{\beta}^{\prime} Y_{-1}\right) \Delta X^{\prime}\left(\Delta X \Delta X^{\prime}\right)^{-1}
$$

e

$$
\hat{\boldsymbol{\Sigma}}_{u}\left(\boldsymbol{\alpha} \boldsymbol{\beta}^{\prime}\right)=\frac{1}{T}\left(\Delta Y \mathbf{M}-\boldsymbol{\alpha} \boldsymbol{\beta}^{\prime} Y_{-1} \mathbf{M}\right)\left(\Delta Y \mathbf{M}-\boldsymbol{\alpha} \boldsymbol{\beta}^{\prime} Y_{-1} \mathbf{M}\right)^{\prime}
$$

Substituindo $\boldsymbol{\Gamma}$ de 2.27 por $\hat{\boldsymbol{\Gamma}}\left(\boldsymbol{\alpha} \boldsymbol{\beta}^{\prime}\right)$ de 2.39 , temos a log-verossimilhança concentrada

$$
\begin{aligned}
\mathscr{L}_{c}(\boldsymbol{\theta})= & -\frac{n T}{2} \ln (2 \pi)-\frac{T}{2} \ln \left(\left|\boldsymbol{\Sigma}_{u}\right|\right)-\frac{1}{2} \operatorname{tr}\left[\left(\Delta Y-\boldsymbol{\alpha} \boldsymbol{\beta}^{\prime} Y_{-1}-\hat{\boldsymbol{\Gamma}}\left(\boldsymbol{\alpha} \boldsymbol{\beta}^{\prime}\right) \Delta X\right)^{\prime} \cdot \boldsymbol{\Sigma}_{u}^{-1} .\right. \\
\cdot & \left.\left(\Delta Y-\boldsymbol{\alpha} \boldsymbol{\beta}^{\prime} Y_{-1}-\hat{\boldsymbol{\Gamma}}\left(\boldsymbol{\alpha} \boldsymbol{\beta}^{\prime}\right) \Delta X\right)\right], \\
= & -\frac{n T}{2} \ln (2 \pi)-\frac{T}{2} \ln \left(\left|\boldsymbol{\Sigma}_{u}\right|\right)-\frac{1}{2} \operatorname{tr}\left[\left(\Delta Y-\boldsymbol{\alpha} \boldsymbol{\beta}^{\prime} Y_{-1}-\left(\Delta Y-\boldsymbol{\alpha} \boldsymbol{\beta}^{\prime} Y_{-1}\right) \Delta X^{\prime}\left(\Delta X \Delta X^{\prime}\right)^{-1} \Delta X\right)^{\prime} \cdot \boldsymbol{\Sigma}_{u}^{-1} .\right. \\
\cdot & \left.\left(\Delta Y-\boldsymbol{\alpha} \boldsymbol{\beta}^{\prime} Y_{-1}-\left(\Delta Y-\boldsymbol{\alpha} \boldsymbol{\beta}^{\prime} Y_{-1}\right) \Delta X^{\prime}\left(\Delta X \Delta X^{\prime}\right)^{-1} \Delta X\right)\right], \\
= & -\frac{n T}{2} \ln (2 \pi)-\frac{T}{2} \ln \left(\left|\boldsymbol{\Sigma}_{u}\right|\right)-\frac{1}{2} \operatorname{tr}\left[(\left(\Delta Y-\boldsymbol{\alpha} \boldsymbol{\beta}^{\prime} Y_{-1}\right) \underbrace{\left(\mathbf{I}_{T}-\Delta X^{\prime}\left(\Delta X \Delta X^{\prime}\right)^{-1} \Delta X\right)}_{\mathbf{M}})^{\prime} \cdot \boldsymbol{\Sigma}_{u}^{-1} .\right. \\
\cdot & \left.(\left(\Delta Y-\boldsymbol{\alpha} \boldsymbol{\beta}^{\prime} Y_{-1}\right) \underbrace{\left(\mathbf{I}_{T}-\Delta X^{\prime}\left(\Delta X \Delta X^{\prime}\right)^{-1} \Delta X\right)}_{\mathbf{M}})\right], \underbrace{\frac{n}{2}} \underbrace{\prime} \ln \left[\left(\Delta Y \mathbf{M}-\boldsymbol{\alpha} \boldsymbol{\beta}^{\prime} Y_{-1} \mathbf{M}\right)^{\prime} \cdot \boldsymbol{\Sigma}_{u}^{-1} \cdot\left(\Delta Y \mathbf{M}-\boldsymbol{\alpha} \boldsymbol{\beta}^{\prime} Y_{-1} \mathbf{M}\right)\right] .
\end{aligned}
$$

A seguir, vamos maximizar 2.41 com relação à $\boldsymbol{\Sigma}_{u}$. Substituindo $\boldsymbol{\Sigma}_{u}$ de 2.41 por $\hat{\boldsymbol{\Sigma}}_{u}\left(\boldsymbol{\alpha} \boldsymbol{\beta}^{\prime}\right)$ de 2.40 , temos que

$$
\mathscr{L}_{c}^{\prime}(\boldsymbol{\theta})=-\frac{n T}{2} \ln (2 \pi)-\frac{T}{2} \ln \left(\left|\hat{\boldsymbol{\Sigma}}_{u}\left(\boldsymbol{\alpha} \boldsymbol{\beta}^{\prime}\right)\right|\right)-\frac{1}{2} \operatorname{tr}[\underbrace{\left(\Delta Y \mathbf{M}-\boldsymbol{\alpha} \boldsymbol{\beta}^{\prime} Y_{-1} \mathbf{M}\right)^{\prime} \cdot \hat{\boldsymbol{\Sigma}}_{u}^{-1}\left(\boldsymbol{\alpha} \boldsymbol{\beta}^{\prime}\right) \cdot\left(\Delta Y \mathbf{M}-\boldsymbol{\alpha} \boldsymbol{\beta}^{\prime} Y_{-1} \mathbf{M}\right)}_{T I_{n}}] .
$$

Note ainda que o termo que está no interior de $\operatorname{tr}[\cdot]$ na Equação 2.42 é igual ao produto de $T$ por uma matriz identidade de ordem $n\left(I_{n}\right)$, pois 


$$
\begin{aligned}
& \left(\Delta Y \mathbf{M}-\boldsymbol{\alpha} \boldsymbol{\beta}^{\prime} Y_{-1} \mathbf{M}\right)^{\prime} \cdot \hat{\boldsymbol{\Sigma}}_{u}^{-1}\left(\boldsymbol{\alpha} \boldsymbol{\beta}^{\prime}\right) \cdot\left(\Delta Y \mathbf{M}-\boldsymbol{\alpha} \boldsymbol{\beta}^{\prime} Y_{-1} \mathbf{M}\right)= \\
& \left(\Delta Y \mathbf{M}-\boldsymbol{\alpha} \boldsymbol{\beta}^{\prime} Y_{-1} \mathbf{M}\right)^{\prime} \cdot\left(\frac{1}{T}\left(\Delta Y \mathbf{M}-\boldsymbol{\alpha} \boldsymbol{\beta}^{\prime} Y_{-1} \mathbf{M}\right)\left(\Delta Y \mathbf{M}-\boldsymbol{\alpha} \boldsymbol{\beta}^{\prime} Y_{-1} \mathbf{M}\right)^{\prime}\right)^{-1} \cdot\left(\Delta Y \mathbf{M}-\boldsymbol{\alpha} \boldsymbol{\beta}^{\prime} Y_{-1} \mathbf{M}\right)= \\
& T \underbrace{\left(\Delta Y \mathbf{M}-\boldsymbol{\alpha} \boldsymbol{\beta}^{\prime} Y_{-1} \mathbf{M}\right)^{\prime} \cdot\left(\Delta Y \mathbf{M}-\boldsymbol{\alpha} \boldsymbol{\beta}^{\prime} Y_{-1} \mathbf{M}\right)^{\prime}-1}_{I_{n}} \cdot \underbrace{\left(\Delta Y \mathbf{M}-\boldsymbol{\alpha} \boldsymbol{\beta}^{\prime} Y_{-1} \mathbf{M}\right)^{-1} \cdot\left(\Delta Y \mathbf{M}-\boldsymbol{\alpha} \boldsymbol{\beta}^{\prime} Y_{-1} \mathbf{M}\right)}_{I_{n}}=T I_{n} .
\end{aligned}
$$

Consequentemente, usando 2.43 a Equação 2.42 pode ser reescrita como

$$
\begin{aligned}
\mathscr{L}_{c}^{\prime}(\boldsymbol{\theta}) & =-\frac{n T}{2} \ln (2 \pi)-\frac{T}{2} \ln \left(\left|\frac{1}{T}\left(\Delta Y \mathbf{M}-\boldsymbol{\alpha} \boldsymbol{\beta}^{\prime} Y_{-1} \mathbf{M}\right)\left(\Delta Y \mathbf{M}-\boldsymbol{\alpha} \boldsymbol{\beta}^{\prime} Y_{-1} \mathbf{M}\right)^{\prime}\right|\right)-\frac{1}{2} \operatorname{tr}\left(T I_{n}\right) \\
& =\underbrace{-\frac{n T}{2} \ln (2 \pi)-\frac{T n}{2}}_{\text {escalar }}-\frac{T}{2} \ln \left(\left|\frac{1}{T}\left(\Delta Y \mathbf{M}-\boldsymbol{\alpha} \boldsymbol{\beta}^{\prime} Y_{-1} \mathbf{M}\right)\left(\Delta Y \mathbf{M}-\boldsymbol{\alpha} \boldsymbol{\beta}^{\prime} Y_{-1} \mathbf{M}\right)^{\prime}\right|\right) .
\end{aligned}
$$

Desconsiderando a soma de termos escalares em 2.44, para maximizar $\mathscr{L}_{c}^{\prime}(\boldsymbol{\theta})$ temos que maximizar

$$
-\frac{T}{2} \ln \left(\left|\frac{1}{T}\left(\Delta Y \mathbf{M}-\boldsymbol{\alpha} \boldsymbol{\beta}^{\prime} Y_{-1} \mathbf{M}\right)\left(\Delta Y \mathbf{M}-\boldsymbol{\alpha} \boldsymbol{\beta}^{\prime} Y_{-1} \mathbf{M}\right)^{\prime}\right|\right),
$$

ou equivalentemente, minimizar

$$
\left|\frac{1}{T}\left(\Delta Y \mathbf{M}-\boldsymbol{\alpha} \boldsymbol{\beta}^{\prime} Y_{-1} \mathbf{M}\right)\left(\Delta Y \mathbf{M}-\boldsymbol{\alpha} \boldsymbol{\beta}^{\prime} Y_{-1} \mathbf{M}\right)^{\prime}\right|
$$

com respeito a $\boldsymbol{\alpha}$ e $\boldsymbol{\beta}$.

Para demonstrar este resultado, vamos aplicar a Proposição A.1 em 2.46, tomando $Y=\Delta Y \mathbf{M}, X=Y_{-1} \mathbf{M}, A=\boldsymbol{\alpha}$ e $B=\boldsymbol{\beta}^{\prime}$. Além disso, vamos considerar as matrizes de covariância amostrais $\mathbf{S}_{i, j}$ definidas em 2.31. Neste caso, de A.1, temos que

$$
\begin{aligned}
\hat{\boldsymbol{\beta}}^{\prime} & =\left[\begin{array}{llll}
\hat{\boldsymbol{v}}_{1} & \hat{\boldsymbol{v}}_{2} & \ldots & \hat{\boldsymbol{v}}_{r}
\end{array}\right]^{\prime}\left(X X^{\prime}\right)^{-\frac{1}{2}} \\
& =\left[\begin{array}{llll}
\hat{\boldsymbol{v}}_{1} & \hat{\boldsymbol{v}}_{2} & \ldots & \hat{\boldsymbol{v}}_{r}
\end{array}\right]^{\prime}\left(\left(Y_{-1} \mathbf{M}\right)\left(Y_{-1} \mathbf{M}\right)^{\prime}\right)^{-\frac{1}{2}} \\
& =\left[\begin{array}{llll}
\hat{\boldsymbol{v}}_{1} & \hat{\boldsymbol{v}}_{2} & \ldots & \hat{\boldsymbol{v}}_{r}
\end{array}\right]^{\prime}\left(R_{1} R_{1}^{\prime}\right)^{-\frac{1}{2}} \\
& =\left[\begin{array}{llll}
\hat{\boldsymbol{v}}_{1} & \hat{\boldsymbol{v}}_{2} & \ldots & \hat{\boldsymbol{v}}_{r}
\end{array}\right]^{\prime} \mathbf{S}_{11}^{-\frac{1}{2}}
\end{aligned}
$$

Além disso, também por A.1, temos que: 


$$
\begin{aligned}
\hat{\boldsymbol{\alpha}} & =Y X^{\prime} \hat{B}^{\prime}\left(\hat{B} X X^{\prime} \hat{B}^{\prime}\right)^{-1} \\
& =(\Delta Y \mathbf{M})\left(Y_{-1} \mathbf{M}\right)^{\prime} \hat{\boldsymbol{\beta}}\left(\hat{\boldsymbol{\beta}}^{\prime}\left(Y_{-1} \mathbf{M}\right)\left(Y_{-1} \mathbf{M}\right)^{\prime} \hat{\boldsymbol{\beta}}\right)^{-1} \\
& =R_{0} R_{1}^{\prime} \hat{\boldsymbol{\beta}}\left(\hat{\boldsymbol{\beta}}^{\prime} R_{1} R_{1}^{\prime} \hat{\boldsymbol{\beta}}\right)^{-1} \\
& =S_{01} \hat{\boldsymbol{\beta}}\left(\hat{\boldsymbol{\beta}}^{\prime} S_{11} \hat{\boldsymbol{\beta}}\right)^{-1}
\end{aligned}
$$

Estimamos em seguida os demais parâmetros do modelo, substituindo $\hat{\boldsymbol{\beta}}$ e $\hat{\boldsymbol{\alpha}}$ das Equações 2.47 e 2.48 nas Equações 2.39 e 2.40. Desta forma, temos:

$$
\hat{\boldsymbol{\Gamma}}=\left(\Delta Y-\hat{\boldsymbol{\alpha}} \hat{\boldsymbol{\beta}}^{\prime} Y_{-1}\right) \Delta X^{\prime}\left(\Delta X \Delta X^{\prime}\right)^{-1}
$$

$\mathrm{e}$

$$
\hat{\boldsymbol{\Sigma}}_{u}=\frac{1}{T}\left(\Delta Y \mathbf{M}-\hat{\boldsymbol{\alpha}} \hat{\boldsymbol{\beta}}^{\prime} Y_{-1} \mathbf{M}\right)\left(\Delta Y \mathbf{M}-\hat{\boldsymbol{\alpha}} \hat{\boldsymbol{\beta}}^{\prime} Y_{-1} \mathbf{M}\right)^{\prime}
$$

Substituindo $\mathbf{M}=\mathbf{I}_{T}-\Delta X^{\prime}\left(\Delta X \Delta X^{\prime}\right)^{-1} \Delta X$ no termo $\left(\Delta Y \mathbf{M}-\hat{\boldsymbol{\alpha}} \hat{\boldsymbol{\beta}}^{\prime} Y_{-1} \mathbf{M}\right)$ da Equação 2.50, nos dá:

$$
\begin{aligned}
\left(\Delta Y \mathbf{M}-\hat{\boldsymbol{\alpha}} \hat{\boldsymbol{\beta}}^{\prime} Y_{-1} \mathbf{M}\right) & =\Delta Y\left(\mathbf{I}_{T}-\Delta X^{\prime}\left(\Delta X \Delta X^{\prime}\right)^{-1} \Delta X\right)-\hat{\boldsymbol{\alpha}} \hat{\boldsymbol{\beta}}^{\prime} Y_{-1}\left(\mathbf{I}_{T}-\Delta X^{\prime}\left(\Delta X \Delta X^{\prime}\right)^{-1} \Delta X\right) \\
& =\Delta Y-\hat{\boldsymbol{\alpha}} \hat{\boldsymbol{\beta}}^{\prime} Y_{-1}-\underbrace{\left(\Delta Y-\hat{\boldsymbol{\alpha}} \hat{\boldsymbol{\beta}}^{\prime} Y_{-1}\right) \Delta X^{\prime}\left(\Delta X \Delta X^{\prime}\right)^{-1}}_{\hat{\boldsymbol{\Gamma}}} \Delta X \\
& =\Delta Y-\hat{\boldsymbol{\alpha}} \hat{\boldsymbol{\beta}}^{\prime} Y_{-1}-\hat{\boldsymbol{\Gamma}} \Delta X .
\end{aligned}
$$

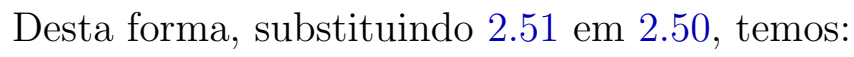

$$
\hat{\boldsymbol{\Sigma}}_{u}=\frac{1}{T}\left(\Delta Y-\hat{\boldsymbol{\alpha}} \hat{\boldsymbol{\beta}}^{\prime} Y_{-1}-\hat{\boldsymbol{\Gamma}} \Delta X\right)\left(\Delta Y-\hat{\boldsymbol{\alpha}} \hat{\boldsymbol{\beta}}^{\prime} Y_{-1}-\hat{\boldsymbol{\Gamma}} \Delta X\right)^{\prime}
$$

Pelo Apêndice A.1, o mínimo da expressão 2.46 é dado por:

$$
\min _{\boldsymbol{\alpha}, \boldsymbol{\beta}}\left|\frac{1}{T}\left(\Delta Y \mathbf{M}-\boldsymbol{\alpha} \boldsymbol{\beta}^{\prime} Y_{-1} \mathbf{M}\right)\left(\Delta Y \mathbf{M}-\boldsymbol{\alpha} \boldsymbol{\beta}^{\prime} Y_{-1} \mathbf{M}\right)^{\prime}\right|=\left|\frac{1}{T}(\Delta Y \mathbf{M})(\Delta Y \mathbf{M})^{\prime}\right| \prod_{i=1}^{r} \ln \left(1-\lambda_{i}\right),
$$

que é equivalente a maximizar 2.46.

Substituindo 2.53 em 2.44, temos que o máximo da log-verossimilhança é atingido em: 


$$
\begin{aligned}
\mathscr{L}_{c}^{\prime}(\boldsymbol{\theta}) & =-\frac{n T}{2} \ln (2 \pi)-\frac{n T}{2}-\frac{T}{2} \ln \left(\left|\frac{1}{T}(\Delta Y \mathbf{M})(\Delta Y \mathbf{M})^{\prime}\right| \prod_{i=1}^{r} \ln \left(1-\lambda_{i}\right)\right) \\
& =-\frac{n T}{2} \ln (2 \pi)-\frac{n T}{2}-\frac{T}{2} \ln \left(\left|\mathbf{S}_{00}\right| \prod_{i=1}^{r} \ln \left(1-\lambda_{i}\right)\right) \\
& =-\frac{T}{2} \sum_{i=1}^{r} \ln \left(1-\lambda_{i}\right)-\frac{T}{2} \ln \left(\left|\mathbf{S}_{00}\right|\right)-\frac{n T}{2} \ln (2 \pi)-\frac{n T}{2} \\
& =-\frac{n T}{2} \ln 2 \pi-\frac{T}{2}\left[\ln \left|\mathbf{S}_{00}\right|+\sum_{i=1}^{r} \ln \left(1-\lambda_{i}\right)\right]-\frac{n T}{2},
\end{aligned}
$$

conforme queríamos demonstrar em 2.38 .

\subsection{Paridade de Poder de Compra}

Seguimos de perto a definição de PPC, enunciadas em Patel (1990), Hayashi (2000) e Falk e Wang (2003). De acordo com os autores, a teoria da PPC afirma que para dois países quaisquer, a taxa de câmbio é igual à razão entre os níveis de preços dos dois países. Conforme aponta Rogoff (1996), a PPC é uma proposição empírica simples atestando que, uma vez convertido em uma moeda comum, os níveis de preços nacionais devem ser iguais.

Seja $P_{t}$ representando o índice de preços do país local, $P_{t}^{*}$ representando o índice de preços para o país estrangeiro e $S_{t}$ representando a taxa de câmbio em moeda do país local por unidade da moeda estrangeira. A teoria da PPC afirma que

$$
P_{t}=S_{t} \cdot P_{t}^{*} .
$$

Tomando o logaritmo em ambos os membros da Equação 2.55, temos que

$$
p_{t}=s_{t}+p_{t}^{*},
$$

$\operatorname{com} p_{t}=\log P_{t}, s_{t}=\log S_{t}$ e $p_{t}^{*}=\log P_{t}^{*}$.

Tanto a Equação 2.55 quanto a Equação 2.56 se referem à paridade de poder de compra "absoluta". A chamada paridade de poder de compra "relativa" exige apenas que a primeira diferença da Equação 2.56 se mantenha, isto é, a taxa de crescimento da taxa de câmbio deve compensar o diferencial entre a taxa de crescimento dos preços domésticos e estrangeiros (Falk e Wang, 2003).

Devido a uma variedade de fatores que limitam a arbitragem de preços internacionais, a PPC não se sustenta exatamente em cada instante de tempo $t$. Desta forma, conforme aponta Hayashi (2000), costuma-se trabalhar com uma versão mais fraca da PPC, dada por 


$$
s_{t}=p_{t}-p_{t}^{*}+u_{t}
$$

com $u_{t}$ sendo um processo estacionário e que considera desvios de curto prazo da PPC.

Mesmo que esta versão não se cumpra no curto prazo, a arbitragem internacional deverá garantir que a hipótese da PPC se cumpra no longo prazo. Além disso, se $s_{t}, p_{t}$ e $p_{t}^{*}$ são processos $I(1)$, então estas séries serão cointegradas com vetor de cointegração igual a

$$
\beta=\left[\begin{array}{lll}
1 & -1 & 1
\end{array}\right]^{\prime}
$$

Falk e Wang (2003) por outro lado, tratam a Equação 2.57 como a versão forte da PPC, trabalhando então com uma forma mais versátil da hipótese, enunciada em Patel (1990) e descrita como

$$
\beta_{0} s_{t}=\eta+\beta_{1} p_{t}+\beta_{2} p_{t}^{*}+u_{t}
$$

onde $u_{t}$ também é um processo estacionário como em 2.57 e os $\beta$ 's são constantes irrestritas.

Neste caso, assumindo também que $s_{t}, p_{t}$ e $p_{t}^{*}$ são processos $I(1)$, temos que as séries são cointegradas, assim como em 2.57. A vantagem da Equação 2.59 é não restringirmos o vetor de cointegração. Por outro lado, quando tomamos $\beta_{1}=-\beta_{2}=1$ (simetria de preços), $\eta=0$ (homogeneidade) e $\beta_{0}=1$ (modelo normalizado), recuperamos a versão absoluta da PPC dada em 2.57. Além disso, conforme aponta Patel (1990), a desvantagem de utilizar a hipótese da PPC apresentada na Equação 2.57 é que esta pode não se manter devido a diferenças na construção dos índices de preços dos países, custos de transação, tarifas, entre outros.

Podemos reescrever a Equação 2.59 como em 2.55, levando em consideração o termo $u_{t}$ :

$$
\begin{aligned}
\beta_{0} \log S_{t} & =\eta+\beta_{1} \log P_{t}+\beta_{2} \log P_{t}^{*}+u_{t} \Rightarrow \\
\log \left[S_{t}^{\beta_{0}}\right] & =\eta+\log \left[P_{t}^{\beta_{1}}\right]+\log \left[P_{t}^{* \beta_{2}}\right]+u_{t} \Rightarrow \\
S_{t}^{\beta_{0}} & =e^{\eta} \cdot P_{t}^{\beta_{1}} \cdot P_{t}^{* \beta_{2}} \cdot e^{u_{t}} .
\end{aligned}
$$

Vamos supor inicialmente o modelo normalizado $\left(\beta_{0}=1\right)$ e simetria de preços $\left(\beta_{1}=\right.$ $-\beta_{2}=1$ ). Por outro lado, vamos supor homogeneidade, ou seja $\eta=0$. Logo, substituindo os valores $\beta_{0}=1, \beta_{1}=1, \beta_{2}=-1$ e $\eta=0$ na última Equação de 2.60, temos que

$$
S_{t}=e^{\eta} \cdot\left(P_{t}\right) \cdot\left(P_{t}^{*}\right)^{-1} \cdot e^{u_{t}} .
$$

Desta forma, rearranjando os termos de 2.61, obtivemos a expressão 


$$
\underbrace{\left(\frac{S_{t}}{e^{u_{t}}} \cdot \frac{P_{t}^{*}}{P_{t}}\right)}_{E_{t}}=e^{\eta}
$$

A Figura 2.1 mostra um exemplo de esquema para a quantidade $e^{\eta}$ teórica e estimada $e^{\hat{\eta}}$. Para este modelo, devemos esperar que a quantidade $e^{\eta}$ oscile em torno do valor teórico igual a 1. Qualquer desvio deste referencial indica a necessidade de considerarmos o intercepto no modelo, ou seja, $\eta \neq 0$.

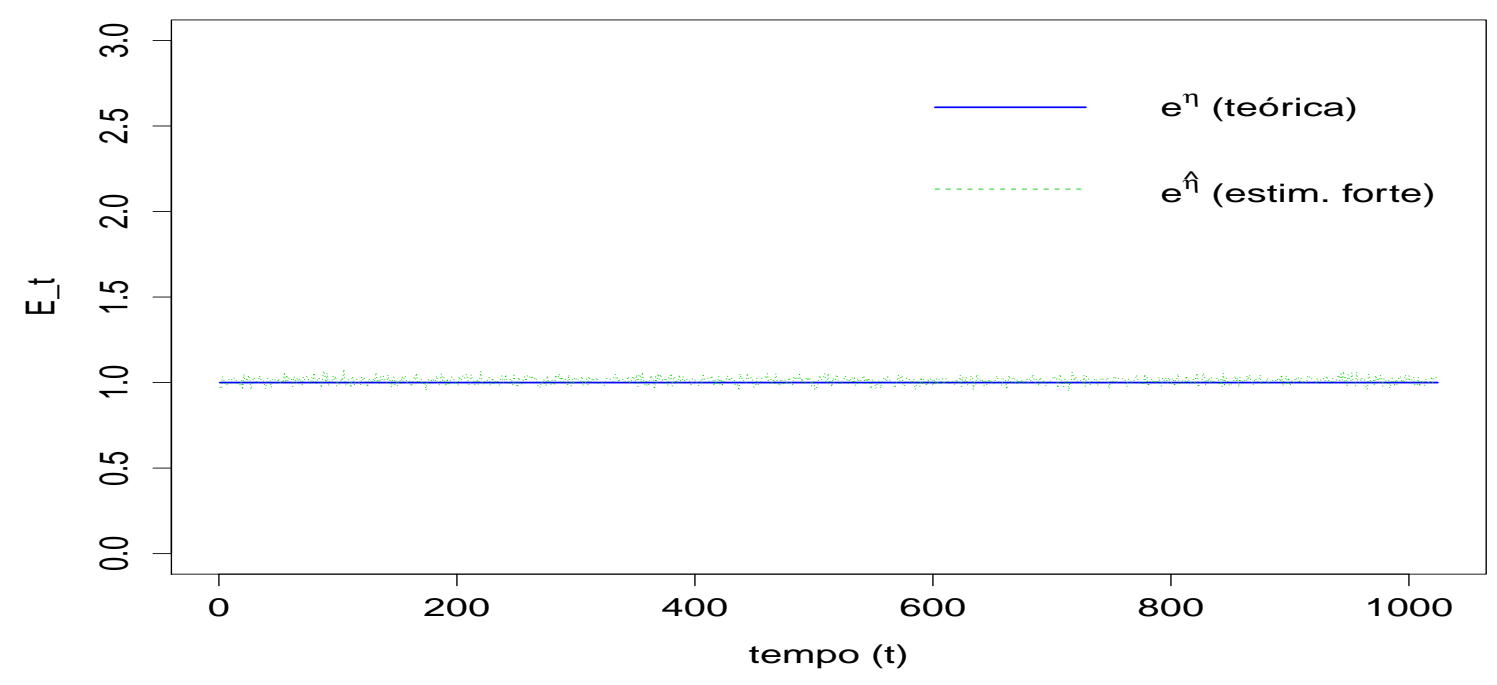

Figura 2.1: Esquema PPC teórica versus estimada.

Outra opção é reescrever a Equação 2.59 de maneira similar à apresentada na Equação 2.12. Neste caso no entanto, vamos tomar $\boldsymbol{\alpha}$ e $\boldsymbol{\beta}$ como sendo matrizes fixas de ordem $(n \times r)$.

Além disso, sem perda de generalidade, vamos considerar a matriz de observações $\Delta Y_{t}$ tomando $n=3, r=1$ e $t=1, \ldots, T$ :

$$
Y_{t}=\left[\begin{array}{c}
Y_{1 t} \\
Y_{2 t} \\
Y_{3 t}
\end{array}\right]=\left[\begin{array}{c}
s_{t} \\
p_{t} \\
p_{t}^{*}
\end{array}\right] \text { e } \Delta Y_{t}=\left[\begin{array}{c}
\Delta Y_{1 t} \\
\Delta Y_{2 t} \\
\Delta Y_{3 t}
\end{array}\right]=\left[\begin{array}{c}
\Delta s_{t} \\
\Delta p_{t} \\
\Delta p_{t}^{*}
\end{array}\right]
$$

Podemos então escrever o modelo dado na Equação 2.59 na forma de correção de erros, conforme descrito na Equação 2.12:

$$
\Delta Y_{t}=\boldsymbol{\alpha} \boldsymbol{\beta}^{\prime} Y_{t-1}+\Gamma \Delta X_{t-1}+u_{t}
$$

Note que para o modelo apresentado em 2.64, estamos desconsiderando o intercepto $\eta$ no modelo. Logo, para estimar os parâmetros de um modelo cointegrado, basta utilizar os procedimentos apresentados na Seção 2.4. O caso com intercepto é tratado de maneira detalhada em Lutkepohl (2005). 


\section{Capítulo 3}

\section{Representação de sinais utilizando ondaletas}

\section{$3.1 \quad$ Ondaletas}

Conforme aponta Nason (2008), como o próprio nome sugere, as ondaletas são "pequenas ondas" que oscilam em determinada região, mas decaem rapidamente para zero. Por meio das ondaletas, podemos representar uma grande quantidade de funções de maneira esparsa, incluindo aquelas que apresentam descontinuidade. Elas nos permitem fazer um "zoom" na função analisada, possibilitando a visualização e a manipulação de escalas. As ondaletas ainda nos permitem detectar, criar e representar características localizadas de funções de interesse.

As ondaletas são funções que satisfazem determinadas propriedades. Elas podem ser suaves (ou não), simétricas (ou não) e podem ter expressões matemáticas simples (ou não), dependendo da base de ondaletas utilizada. Além disso, elas são localizadas no tempo ou espaço. Este comportamento torna estas funções ideais para analisar sinais não estacionários, permitindo aproximar uma função qualquer por meio de uma combinação linear de ondaletas (Morettin, 2014). As caracteristicas descritas acima são extremamente úteis para o presente trabalho, uma vez que vamos considerar o vetor de cointegração variando com tempo, sendo desta forma, assumido como sendo não constante.

Conforme aponta Shumway e Stoffer (2011), as ondaletas são caracterizadas em família, sendo geradas por uma ondaleta pai $\phi$ e uma ondaleta mãe $\psi$. A ondaleta pai é utilizada para capturar a natureza suave e de baixa frequência dos dados, enquanto a ondaleta mãe é utilizada para capturar os detalhes e a natureza de alta frequência dos dados. A ondaleta pai integra um, enquanto a ondaleta mãe integra zero:

$$
\int \phi(t) d t=1 \text { e } \int \psi(t) d t=0 .
$$

De maneira geral, um sinal ou função de quadrado integrável $f(t)$ pode ser representado 
por meio de uma combinação linear de funções de ondaletas (mãe) e funções de escala (pai), para algum $j_{0} \in \mathbb{Z}$ fixo, conforme apresentado na Equação 3.2 (Nason, 2008):

$$
f(t)=\sum_{k \in \mathbb{Z}} c_{j_{0}, k} \phi_{j_{0}, k}(t)+\sum_{j=j_{0}}^{\infty} \sum_{k \in \mathbb{Z}} d_{j, k} \psi_{j, k}(t) .
$$

Conforme aponta Morettin (2014), geralmente não tomamos todos os níveis de resolução $J$. Neste caso, tomamos um valor $J$ que corresponde à escala mais fina $2^{J}$. Assim, reescrevendo a Equação 3.2, temos que

$$
f(t)=\sum_{k=0}^{2^{j_{0}}-1} c_{j_{0}, k} \phi_{j_{0}, 0}(t)+\sum_{j=j_{0}}^{J} \sum_{k=0}^{2^{j}-1} d_{j, k} \psi_{j, k}(t),
$$

onde $2^{j_{0}}$ indica o número de escalas para a ondaleta pai e $J=J_{\max }-1$ representa o nível de detalhe desejado, sendo $J_{\max }$ o nível máximo permitido para o conjunto de dados diático utilizado (Nason, 2008; Morettin, 2014).

Note que ao tomar $j_{0}=0$ na Equação 3.3, o sinal $f(t)$ pode ser reescrito de maneira conveniente, com apenas uma escala para a ondaleta pai:

$$
f(t)=c_{0,0} \phi_{0,0}(t)+\sum_{j=0}^{J} \sum_{k=0}^{2^{j}-1} d_{j, k} \psi_{j, k}(t) .
$$

Além disso, os coeficientes $c_{0,0}$ e $d_{j, k}$ na Equação 3.4 devem ser estimados.

\subsection{Famílias de ondaletas}

Existe na literatura uma vasta quantidade de bases de ondaletas. Diversas delas são apresentadas em Mallat (1999), Nason (2008), Shumway e Stoffer (2011), Morettin (2014), entre outros. A seguir, apresentamos algumas destas bases, empregadas no presente trabalho.

\subsubsection{Família de ondaletas Haar}

A função de $\psi(t)$ definida em 3.5 é chamada de ondaleta mãe na base Haar. Esta ondaleta dá origem a uma família inteira de ondaletas por meio de duas operações: dilatações diádicas e translações inteiras.

$$
\psi(t)=\left\{\begin{array}{lll}
1 & , \text { se } & t \in\left[0 ; \frac{1}{2}\right) \\
-1 & , \text { se } & t \in\left[\frac{1}{2} ; 1\right.
\end{array}\right) .
$$

Denotamos $j$ como o indice de dilatação e $k$ o índice de translação. Cada ondaleta 
"gerada" pela ondaleta mãe é indexada pelos dois índices, conforme indicado em 3.6:

$$
\psi_{j, k}(t)=\left\{\begin{array}{lll}
2^{\frac{j}{2}} & , \text { se } & t \in\left[2^{-j} k ; 2^{-j}\left(k+\frac{1}{2}\right)\right) \\
-2^{\frac{j}{2}} & , \text { se } & t \in\left[2^{-j}\left(k+\frac{1}{2}\right) ; 2^{-j}(k+1)\right) . \\
0 & \text {, caso contráro. } &
\end{array}\right.
$$

A ondaleta pai na base Haar por sua vez, será dada por:

$$
\phi_{j, k}(t)=\left\{\begin{array}{ll}
2^{\frac{j}{2}} & , \text { se } \\
0 & , \text { caso contrário }
\end{array} t \in\left[2^{-j} k ; 2^{-j}(k+1)\right) .\right.
$$

Na Figura 3.1, apresentamos a representação gráfica destas funções para esta base de ondaletas.
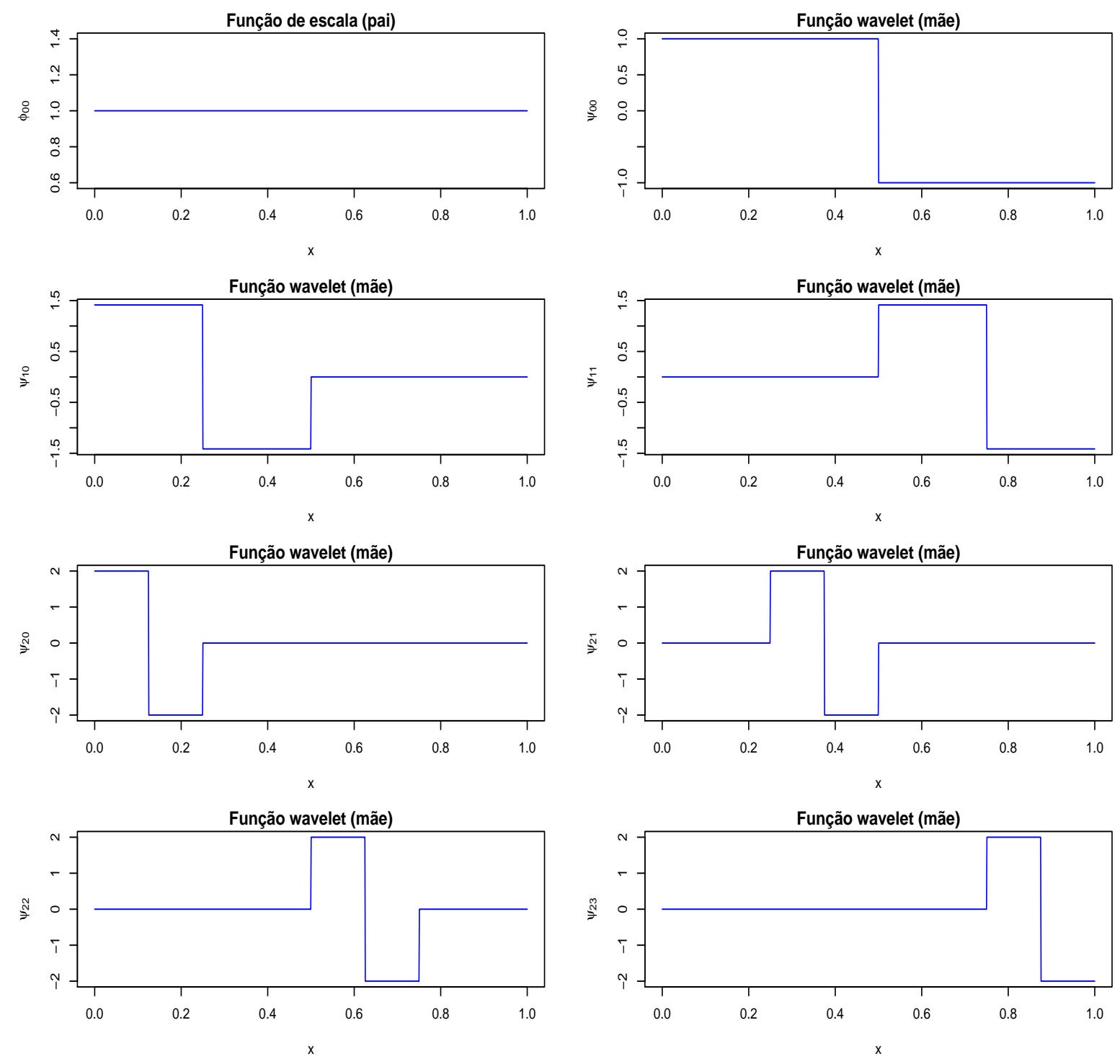

Figura 3.1: Comportamento da ondaleta Haar pai $\phi_{00}(t)$ e mãe $\psi_{j k}(t)$, para $j=0,1$ e 2 . 


\subsubsection{Família de ondaletas Daublet}

Conforme apontam Bruce e Gao (1996), as ondaletas Daublet foram as primeiras ondaletas ortogonais contínuas com suporte compacto. Elas receberam este nome como homenagem à sua criadora, Ingrid Daubechies. Estas ondaletas são bastante assimétricas.
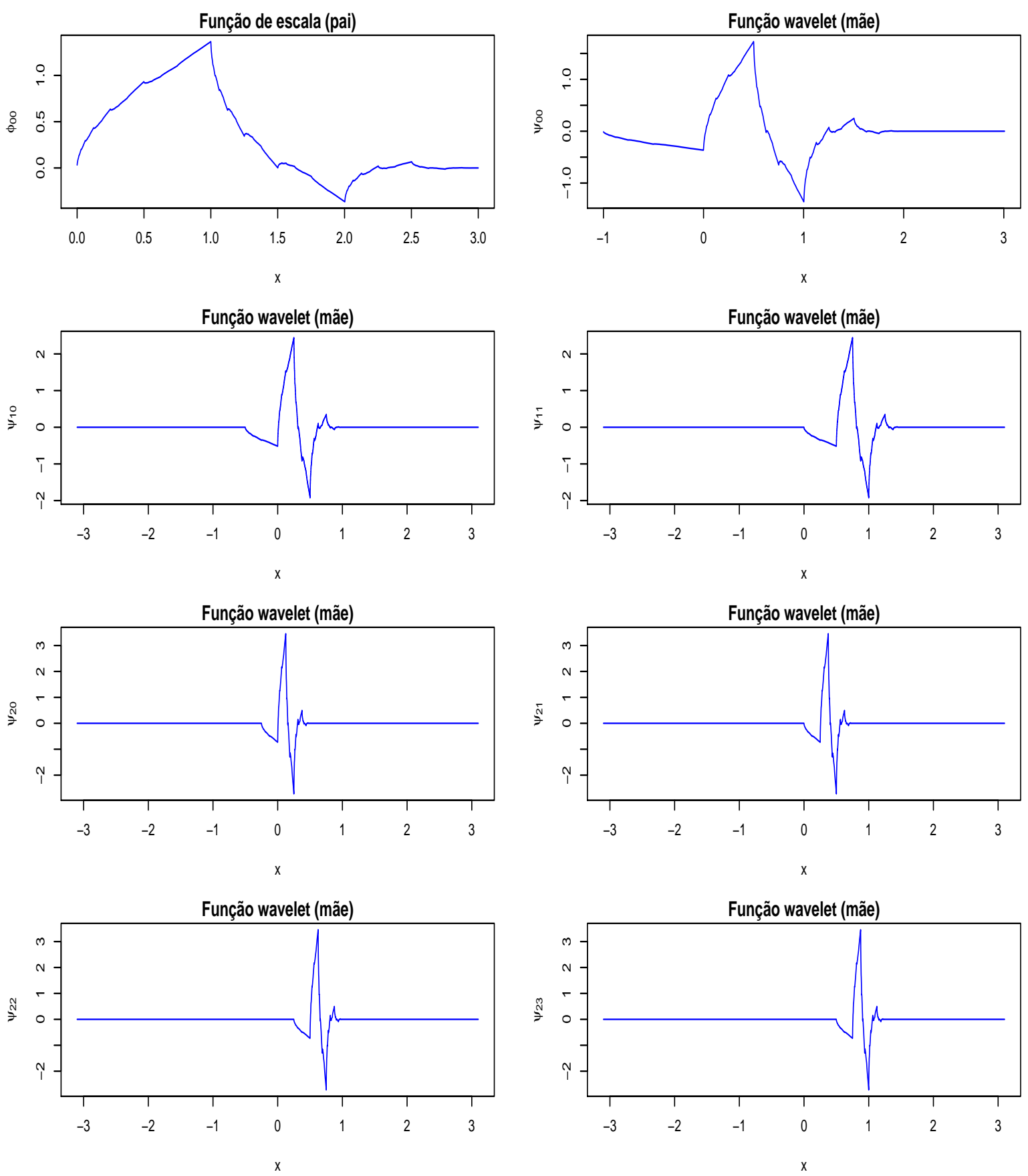

Figura 3.2: Comportamento da ondaleta Daublet D2 pai $\phi_{00}(t)$ e mãe $\psi_{j k}(t)$, para $j=0,1$ e 2 . 


\subsubsection{Família de ondaletas Symmlet}

As ondaletas Symmlet também possuem suporte compacto e foram construídas para serem aproximadamente simétricas, ou menos assimétricas possíveis (Bruce e Gao, 1996).
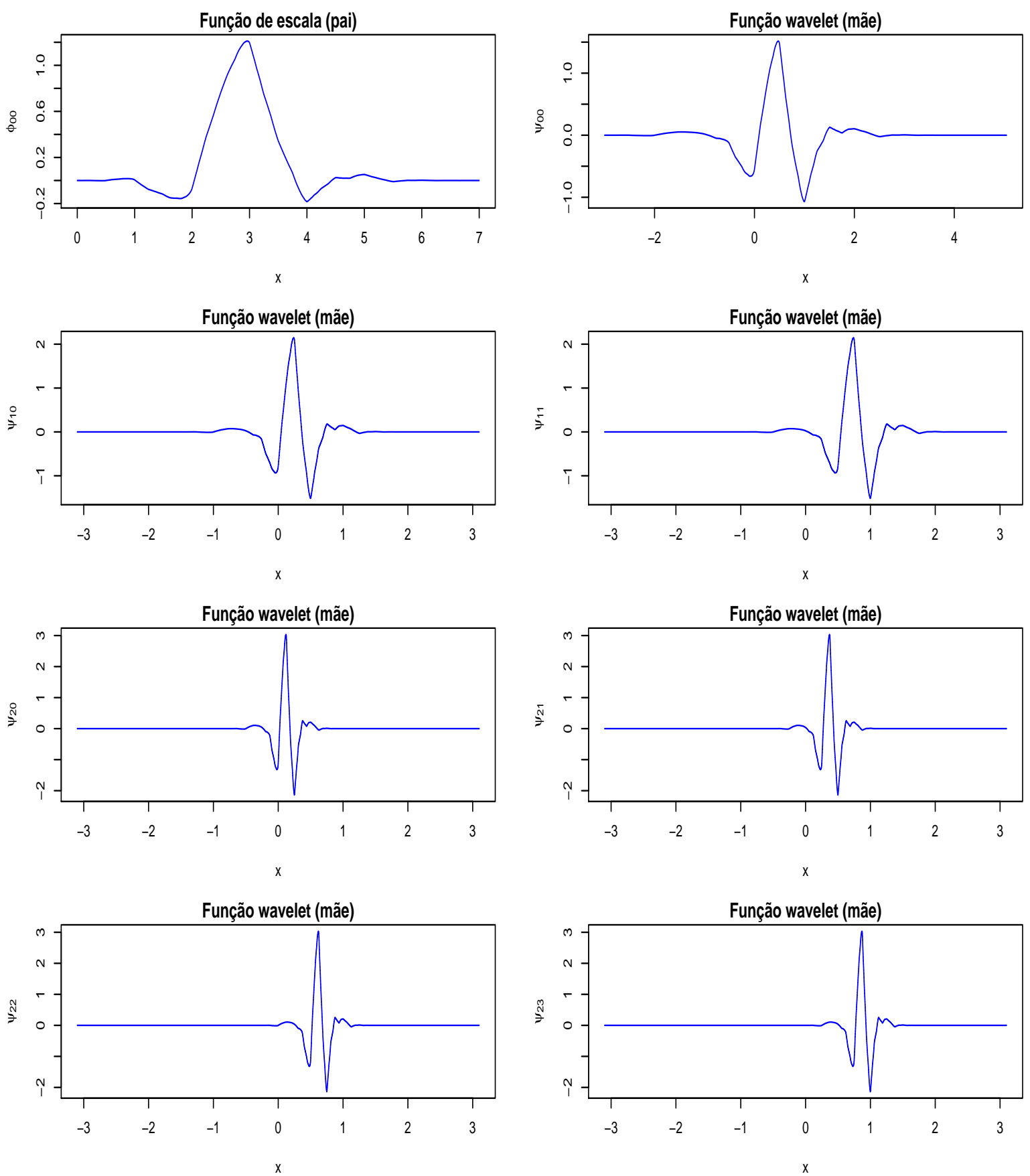

Figura 3.3: Comportamento da ondaleta Symmlet S4 pai $\phi_{00}(t)$ e mãe $\psi_{j k}(t)$, para $j=0,1$ e 2 . 


\subsection{Limiarização de ondaletas - contexto univariado}

O conceito de limiarização (Morettin, 2014) ou thresholding foi introduzido nos artigos seminais de Donoho (1993), Donoho e Johnstone (1994) e Donoho et al. (1995). Um dos pontos considerados pelos autores é o fato de que a energia de um sinal é geralmente concentrada em alguns coeficientes da transformada de ondaletas. Logo, um sinal pode ser suavizado ao ser descrito por um número relativamente pequeno de coeficientes.

Conforme aponta Downie e Silverman (1998), um método de suavização adapta os coeficientes de ondaletas utilizando alguma regra que depende da magnitude dos coeficientes. O objetivo é encontrar uma versão mais suave do sinal original.

Para aplicar uma limiarização na transformada de ondaletas, deve-se definir inicialmente o tipo de limiar a ser empregado. Conforme apontam Nason (2008) e Morettin (2014), são comumente utilizados na literatura dois esquemas de limiares:

1. Limiar duro (hard thresholding):

$$
\delta_{H}(x, \lambda)=\left\{\begin{array}{lll}
x & \text {, se } & |x|>\lambda \\
0 & \text {, se } & |x| \leq \lambda
\end{array}\right.
$$

2. Limiar suave (soft thresholding):

$$
\delta_{S}(x, \lambda)=\left\{\begin{array}{lll}
\operatorname{sinal}(x)(|x|-\lambda) & , \text { se } & |x|>\lambda \\
0 & , \text { se } & |x| \leq \lambda
\end{array}\right.
$$

Conforme aponta Morettin (2014), a Equação 3.8 é do tipo "mata ou preserva" enquanto que a Equação 3.9 é do tipo "mata ou encolhe". Apresentamos na Figura 3.4 um exemplo de limiar duro e limiar suave, para $\lambda=1$.

(a)

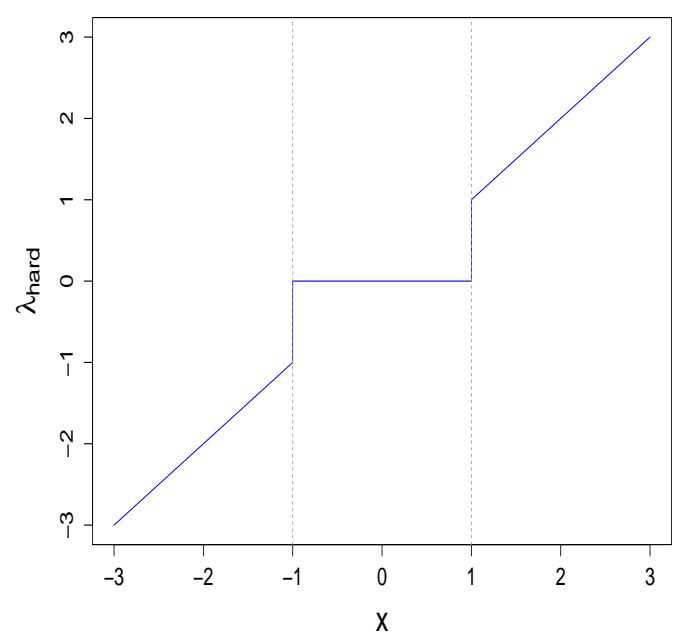

Figura 3.4: (a) Limiar duro (hard) $\operatorname{com} \lambda=1$, (b)

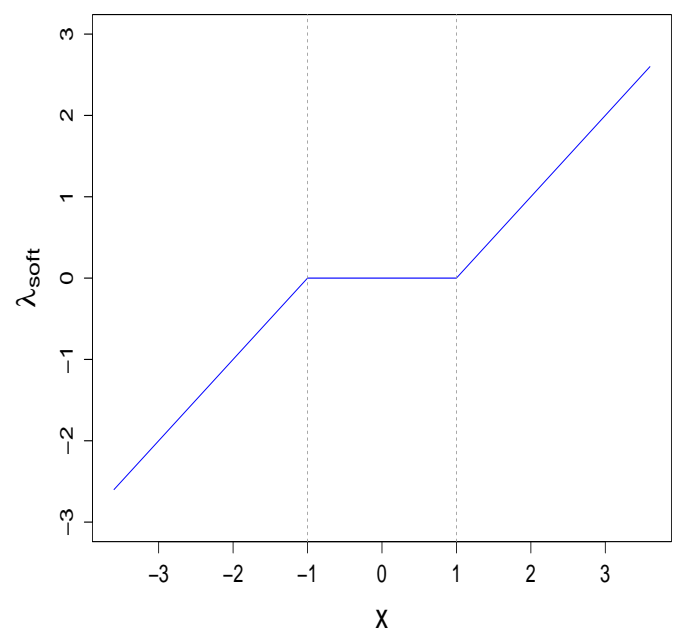

(b) Limiar suave (soft) $\operatorname{com} \lambda=1$. 
Existem na literatura, diversos métodos para a escolha do limiar $\lambda$ (Equações $3.8 \mathrm{e}$ 3.9), conforme descritos em Nason (2008) e Morettin (2014).

Os métodos adaptativos são aqueles que dependem do nível $j$, ou seja, temos um limiar $\lambda_{j}$ diferente para cada escala. Os métodos globais por sua vez são aqueles aplicados a todos os níveis. Um exemplo é o método de limiarização universal, proposto por Donoho e Johnstone (1994). O método é baseado no seguinte resultado:

Proposição 3.1. Sejam $Z_{1}, \ldots, Z_{n}$ variáveis aleatórias normais padrão. Defina:

$$
A_{n}=\left\{\max _{i=(1, \ldots, n)}\left|Z_{i}\right| \leq \sqrt{2 \log (n)}\right\},
$$

logo, $\Pi_{n}=P\left(A_{n}\right) \rightarrow 1, n \rightarrow \infty$. Por outro lado, se

$$
B_{n}(t)=\left\{\max _{i=(1, \ldots, n)}\left|Z_{i}\right|>t+\sqrt{2 \log (n)}\right\},
$$

então $P\left(B_{n}(t)\right)<e^{\frac{-t^{2}}{2}}$.

Demonstração: Este resultado foi demonstrado em Donoho e Johnstone (1994).

Conforme aponta Morettin (2014), o resultado da Proposição 3.1 garante (informalmente) que as variváveis aleatórias $Z_{i}$ 's (independentes ou não) são quase limitadas por $\pm \sqrt{2 \log (n)}$. Logo, com base nos resultados da Proposição 3.1, podemos definir o limiar

$$
\lambda_{n}=\lambda_{j, n}=\sigma \sqrt{2 \log (n)},
$$

que não depende da escala e é chamado de "universal". O termo $\sigma$ é estimado a partir dos dados.

Donoho et al. (1995) definem uma nova versão de 3.12, dado em 3.13

$$
\lambda_{n}=\lambda_{j, n}=\sigma \sqrt{\frac{2 \log (n)}{n}} .
$$

Conforme apresentado em Donoho et al. (1995), estimamos $\sigma$ por meio da Equação 3.14

$$
\hat{\sigma}=\frac{\text { mediana }\left\{\left|d_{J-1, k}\right|: 0 \leq k<2^{J}\right\}}{0,6745}
$$

onde $J-1$ refere-se à escala mais fina. 


\subsection{Limiarização de ondaletas - contexto multivaria- do}

Desejamos aplicar um método de limiarização para o modelo 4.4, utilizando algum método com o apresentado na Seção 3.3 para ondaletas univariadas. No caso multivariado, devemos fazer algumas considerações:

1. Uma vez escolhido o valor máximo para $J$, desejamos excluir os coeficientes que não contribuam para estimar os parâmetros do modelo, ou que causem algum ruído;

2. Devemos considerar que em $\mathbf{W}_{J}$, definido no Capítulo 4, na Equação 4.8, os coeficientes $\mathbf{c}_{0,0}$ e $\mathbf{d}_{j, k}$ são matrizes de ordem $n \times r$, com representação dada na Equação 4.2. Por exemplo, para o caso em que $n=2, r=1$, temos que

$$
\mathbf{d}_{j, k}=\left[\begin{array}{c}
d_{j, 0}^{(1,1)} \\
d_{k, 0}^{(2,1)}
\end{array}\right]_{2 \times 1}
$$

para índices $j=0,1, \ldots, J$ e $k=0,1, \ldots, 2^{J}-1$, sendo $J$ o nível de detalhe desejado. Logo, excluir o coeficiente $\mathbf{d}_{j, k}$ implica desconsiderar as contribuições de $d_{j, k}^{(1,1)}$ e $d_{j, k}^{(2,1)}$ simultaneamente;

3. Assim, antes de aplicar algum método de limiarização, precisamos de uma medida que resuma a contribuição global da escala, de preferência contribuição do nível ao longo do tempo.

Os conceitos apresentados na Seção 3.3 são aplicados costumeiramente no contexto univariado. No entanto, no contexto multivariado (modelo 4.4), os coeficientes de ondaletas para o modelo não são escalares e sim matrizes $(n \times r)$.

Conforme apontam Downie e Silverman (1998), a extensão imediata para aplicar as técnicas de threshold universal a coeficientes de ondaletas múltiplas (caso multivariado) é aplicar as técnicas individualmente para cada coeficiente. Strela et al. (1999) se valeram desta estratégia.

Downie e Silverman (1998) propuseram outra técnica para o contexto multivariado, que se mostrou mais eficiente que a estratégia anterior. Os passos necessários para aplicar o limiar neste contexto são descritos a seguir.

Assim como no caso univariado, precisamos definir inicialmente o tipo de limiar a ser empregado. Para um limiar $\lambda$, são apresentados a seguir os respectivos esquemas de limiares para o caso multivariado: 
1. Limiar duro (hard thresholding):

$$
\delta_{d}\left(\theta_{j, k}, \lambda\right)=\left\{\begin{array}{lll}
\mathbf{d}_{j, k} & , \text { se } & \theta_{j, k} \geq \lambda \\
0 & , \text { se } & \theta_{j, k}<\lambda
\end{array}\right.
$$

2. Limiar suave (soft thresholding):

$$
\delta_{S}\left(\theta_{j, k}, \lambda\right)=\mathbf{d}_{j, k} \cdot\left(\frac{\max \left(\theta_{j, k}-\lambda, 0\right)}{\theta_{j, k}}\right)
$$

Em quaisquer dos limiares utilizados (3.16 ou 3.17), $\theta_{j, k}$ são os valores que deverão ser limiarizados. Para o cálculo de $\theta_{j, k}$, utilizamos decomposição

$$
\theta_{j, k}=\mathbf{d}_{j, k}^{\prime} V_{j}^{-1} \mathbf{d}_{j, k}
$$

Conforme apontam Downie e Silverman (1998), $\theta_{j, k}$ segue uma distribuição qui-quadrado $\chi_{L}^{2}$, onde $L$ representa a quantidade de coeficientes de ondaletas utilizada na decomposição.

Na Equação 3.18, $\mathbf{d}_{j, k}$ são as matrizes de coeficientes, conforme exemplificado em 3.15. Para cada combinação de $j$ e $k$, obtemos um escalar $\theta_{j, k}$, sendo o termo $V_{j}$ a variância dentro da matriz $\mathbf{d}_{j, k}$ :

$$
V_{j}=\operatorname{Var}\left(\mathbf{d}_{j, k}\right)
$$

Nos resta apenas encontrar o limiar $\lambda$ universal para o contexto multivariado. Temos que $\theta_{j, k} \sim \chi_{L}^{2}$. Logo, para encontrar o limiar universal, deve-se encontrar o máximo assintótico de $n$ variáveis aleatórias $\chi_{L}^{2}$ i.i.d.. Este resultado é apresentado no Teorema 3.4.1:

Teorema 3.4.1. Seja $M_{n}$ o máximo de $n$ variáveis aleatórias $\chi_{L}^{2}$ i.i.d., com $L>1$ e seja $\lambda$ o infimo de todas as sequências $a_{n}$ que satisfazem

$$
P\left(M_{n} \leq a_{n}\right) \rightarrow 1, n \rightarrow \infty
$$

Desta forma,

$$
\lambda=2 \log n+(L-2) \log \log n .
$$

Demonstração: Este resultado foi demonstrado em Downie e Silverman (1998).

Quando $L=2$, temos que $\lambda=2 \log n$. No entanto, conforme sugerem os autores, para $L>2$ ainda é conveniente omitir o termo $(L-2) \log \log n$, visto que a Expressão 3.21 superestima o verdadeiro valor do limiar $\lambda$. Desta forma, em todos os casos, utilizamos o seguinte limiar universal para o contexto multivariado: 


$$
\lambda=2 \log n
$$

Assim, uma vez calculados $\theta_{j, k}$ para cada uma das matrizes de coeficientes $\mathbf{d}_{j, k}$, construímos medidas que resumem a contribuição de cada escala ao modelo. Em seguida, é possível compará-los com relação à sua magnitude e excluir aqueles que porventura não contribuem ou causam algum ruído à estimação dos parâmetros do modelo. Basta então comparar os valores dos $\theta_{j, k}$ com o limiar universal obtido na Equação 3.22. Na Figura 3.5 é apresentado um exemplo, onde são plotados os valores de $\theta_{j, k}$ de cada coeficiente juntamente com o limiar. Valores de $\theta_{j, k}$ abaixo do limiar devem ser excluídos do modelo.

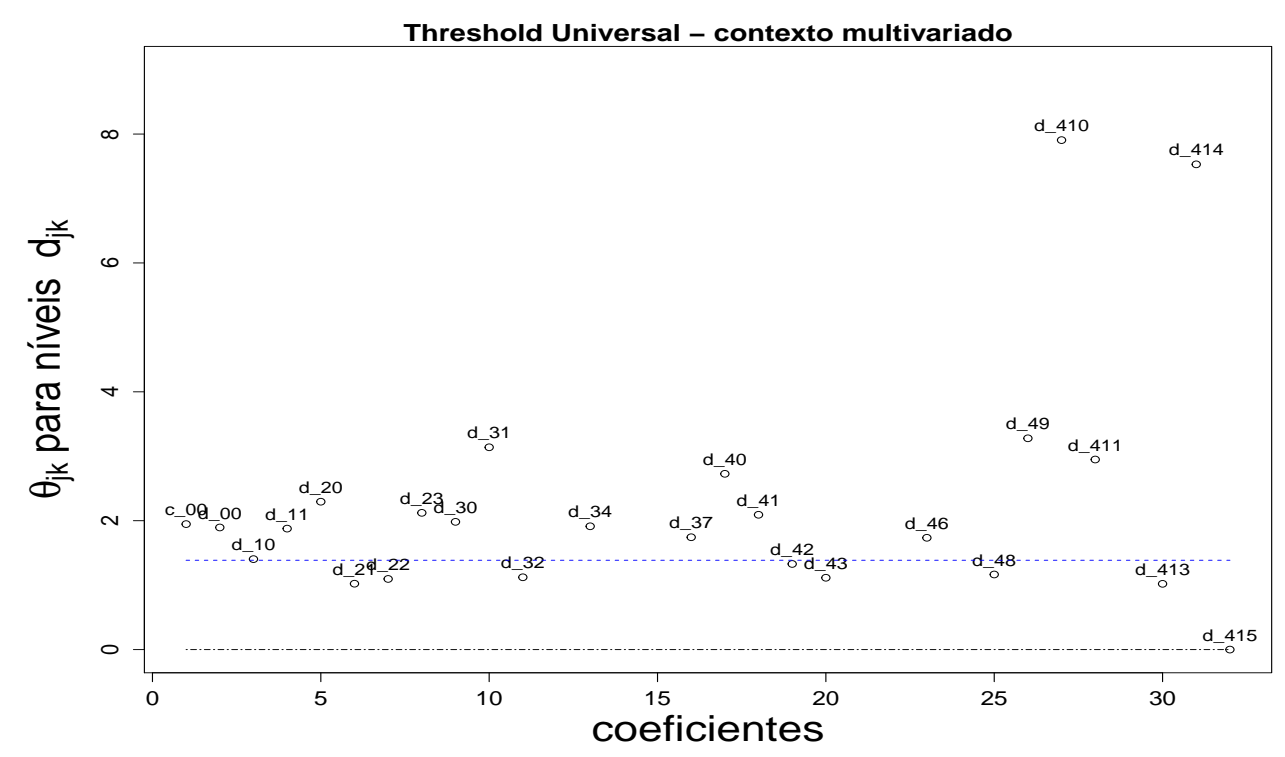

Figura 3.5: Exemplo de threshold universal para o contexto multivariado. Valores de $\theta_{j, k}$ abaixo do limiar devem ser excluídos do modelo. 


\section{Capítulo 4}

\section{Modelo de Correção de Erros Vetorial com vetor de cointegração variando com o tempo}

O modelo básico considerado será o modelo VAR na forma de correção de erros (VEC) com erros gaussianos independentes e identicamente distribuídos (i.i.d.). Assim, como em Johansen (1995), trabalhamos com a hipótese de posto reduzido, ou seja, a hipótese de existência de vetores de cointegração em uma forma paramétrica simples, permitindo a aplicação do método de máxima verossimilhança e testes de razão de verossimilhanças. Neste sentido, vamos seguir a metodologia apresentada em Bierens e Martins (2010), que permite ao vetor de cointegração variar com o tempo. Desta forma, utilizando ondaletas, podemos derivar estimadores para os parâmetros do modelo e a estatística do teste de razão de verossimilhanças para a hipótese de cointegração variando com o tempo.

\subsection{Construção do modelo}

Desejamos generalizar o modelo VEC dado na Equação 2.12, permitindo que o vetor de cointegração $\boldsymbol{\beta}$ do modelo varie com o tempo. Nosso interesse reside em substituir o parâmetro $\boldsymbol{\beta}$ do modelo pela sua representação por meio de ondaletas $\boldsymbol{\beta}_{t}$, para $t=$ $1, \ldots, T$. Utilizando a Equação 3.4 , a expansão em ondaletas do parâmetro $\boldsymbol{\beta}$ do modelo é dada por 


$$
\left.\begin{array}{rl}
\boldsymbol{\beta}_{t} & =\left(\begin{array}{ccc}
\beta_{1,1}(t) & \ldots & \beta_{1, r}(t) \\
\vdots & \ddots & \vdots \\
\beta_{n, 1}(t) & \ldots & \beta_{n, r}(t)
\end{array}\right)_{n \times r} \\
& =\left(\begin{array}{cccc}
c_{0,0}^{(1,1)} \phi(t)+\sum_{j=0}^{J} \sum_{k=0}^{2^{j}-1} d_{j, k}^{(1,1)} \psi_{j, k}(t) & \ldots & c_{0,0}^{(1, r)} \phi(t)+\sum_{j=0}^{J} \sum_{k=0}^{2^{j}-1} d_{j, k}^{(1, r)} \psi_{j, k}(t) \\
\vdots & \ddots & \vdots \\
c_{0,0}^{(n, 1)} \phi(t)+\sum_{j=0}^{J} \sum_{k=0}^{2^{j}-1} d_{j, k}^{(n, 1)} \psi_{j, k}(t) & \ldots & c_{0,0}^{(n, r)} \phi(t)+\sum_{j=0}^{J} \sum_{k=0}^{2^{j}-1} d_{j, k}^{(n, r)} \psi_{j, k}(t)
\end{array}\right) \\
& =\left(\begin{array}{cccc}
\psi_{0,0}(t) & \psi_{1,0}(t) & \ldots & \psi_{J, 2^{J}-1}(t)
\end{array}\right]\left[\begin{array}{c}
\mathbf{c}_{0,0} \\
\mathbf{d}_{0,0} \\
\mathbf{d}_{1,0} \\
\vdots \\
\mathbf{d}_{J, 2^{J}-1}
\end{array}\right]
\end{array}\right)
$$

onde $\psi_{j, k}(t)$ e $\phi(t)$, representam as funções de ondaletas mãe e pai respectivamente. Como exemplo, podemos citar a base Haar, onde estas funções são dadas nas Equações 3.6 e 3.7. Exemplos de funções de outras bases foram apresentadas no Capítulo 3.

Note ainda que as matrizes $\mathbf{c}$ e $\mathbf{d}_{j, k}$, para $j=(1, \ldots, J)$ e $k=\left(1, \ldots, 2^{J}-1\right)$, possuem ordem $(n \times r)$ e carregam os coeficientes de ondaletas para cada nível da decomposição. Neste caso, as matrizes são representadas como

$$
\mathbf{c}_{0,0}=\left[\begin{array}{ccc}
c_{0,0}^{(1,1)} & \ldots & c_{0,0}^{(1, r)} \\
\vdots & \ddots & \vdots \\
c_{0,0}^{(n, 1)} & \ldots & c_{0,0}^{(n, r)}
\end{array}\right]_{n \times r} \quad \text { e } \quad \mathbf{d}_{j, k}=\left[\begin{array}{ccc}
d_{j, k}^{(1,1)} & \ldots & d_{j, k}^{(1, r)} \\
\vdots & \ddots & \vdots \\
d_{j, k}^{(n, r)} & \ldots & d_{j, k}^{(n, r)}
\end{array}\right]_{n \times r}
$$

Assim como fizemos para o modelo VEC, vamos tomar $\boldsymbol{\alpha}$ como uma matriz fixa de ordem $(n \times r)$. Além disso, vamos supor que a matriz $\boldsymbol{\Pi}=\boldsymbol{\Pi}_{t}$ pode ser escrita como $\boldsymbol{\alpha} \boldsymbol{\beta}_{t}^{\prime}$, para todo $t=1, \ldots, T$. Logo, desconsiderando o intercepto, podemos reescrever o modelo VEC da Equação 2.12 como

$$
\begin{aligned}
\Delta Y_{t} & =\boldsymbol{\Pi}_{t} Y_{t-1}+\boldsymbol{\Gamma}_{1} \Delta Y_{t-1}+\ldots+\boldsymbol{\Gamma}_{p-1} \Delta Y_{t-p+1}+u_{t} \\
& =\boldsymbol{\alpha} \boldsymbol{\beta}_{t}^{\prime} Y_{t-1}+\sum_{i=1}^{p-1} \boldsymbol{\Gamma}_{i} \Delta Y_{t-1}+u_{t}
\end{aligned}
$$

Em seguida, substituimos $\boldsymbol{\beta}_{t}$ da Equação 4.1 na Equação 4.3, obtendo modelo VEC com vetor de cointegração decomposto por ondaletas e, consequentemente, variando com 
o tempo. Esse modelo será chamado de WAVEC.

$$
\begin{aligned}
& \Delta Y_{t}=\boldsymbol{\alpha}\left(\left[\begin{array}{lllll}
\phi(t) & \psi_{0,0}(t) & \psi_{1,0}(t) & \ldots & \psi_{J, 2^{J}-1}(t)
\end{array}\right]\left[\begin{array}{c}
\mathbf{c}_{0,0} \\
\mathbf{d}_{0,0} \\
\mathbf{d}_{1,0} \\
\vdots \\
\mathbf{d}_{J, 2^{J}-1}
\end{array}\right]\right)^{\prime} Y_{t-1}+\sum_{i=1}^{p-1} \boldsymbol{\Gamma}_{i} \Delta Y_{t-i}+u_{t} \\
& =\boldsymbol{\alpha}\left[\begin{array}{lllll}
\mathbf{c}_{0,0}^{\prime} & \mathbf{d}_{0,0}^{\prime} & \mathbf{d}_{1,0}^{\prime} & \ldots & \mathbf{d}_{J, 2^{J}-1}^{\prime}
\end{array}\right]\left[\begin{array}{c}
\phi(t) Y_{t-1} \\
\psi_{0,0}(t) Y_{t-1} \\
\psi_{1,0}(t) Y_{t-1} \\
\vdots \\
\psi_{J, 2^{J}-1}(t) Y_{t-1}
\end{array}\right]+\sum_{i=1}^{p-1} \boldsymbol{\Gamma}_{i} \Delta Y_{t-i}+u_{t} \\
& \Delta Y_{t}=\boldsymbol{\alpha} \mathbf{W}_{J}^{\prime} Y_{t-1}^{(J)}+\sum_{i=1}^{p-1} \boldsymbol{\Gamma}_{i} \Delta Y_{t-i}+u_{t} .
\end{aligned}
$$

Reescrevendo a Equação 4.4 em notação matricial, temos:

$$
\Delta Y=\boldsymbol{\alpha} \mathbf{W}_{J}^{\prime} Y_{-1}^{(J)}+\boldsymbol{\Gamma} \Delta X+U
$$

onde

$$
\begin{aligned}
& \Delta Y:=\left[\begin{array}{llll}
\Delta Y_{1} & \Delta Y_{2} & \ldots & \Delta Y_{T}
\end{array}\right] \\
& \boldsymbol{\alpha}=\left[\begin{array}{ccc}
\alpha_{1,1} & \ldots & \alpha_{1, r} \\
\vdots & \ddots & \vdots \\
\alpha_{n, 1} & \ldots & \alpha_{n, r}
\end{array}\right]_{n \times r} \\
& \mathbf{W}_{J}^{\prime}:=\left[\begin{array}{lllll}
\mathbf{c}_{0,0}^{\prime} & \mathbf{d}_{0,0}^{\prime} & \mathbf{d}_{1,0}^{\prime} & \ldots & \mathbf{d}_{J, 2^{J}-1}^{\prime}
\end{array}\right]_{r \times\left(n 2^{J+1}\right)}, \\
& Y_{-1}^{(J)}:=\left[\begin{array}{c}
\phi(t) Y_{-1} \\
\psi_{0,0}(t) Y_{-1} \\
\vdots \\
\psi_{J,\left(2^{J}-1\right)}(t) Y_{-1}
\end{array}\right]_{n 2^{J+1} \times 1} \quad, \text { com } \quad Y_{-1}=\left[\begin{array}{llll}
Y_{0} & Y_{1} & \ldots & Y_{T-1}
\end{array}\right], \\
& \boldsymbol{\Gamma}=\left[\begin{array}{lll}
\boldsymbol{\Gamma}_{1} & \ldots & \boldsymbol{\Gamma}_{p-1}
\end{array}\right]_{n \times n(p-1)},
\end{aligned}
$$




$$
\begin{gathered}
\Delta X=\left[\begin{array}{llll}
\Delta X_{0} & \Delta X_{1} & \ldots & \Delta X_{T-1}
\end{array}\right], \operatorname{com} \quad \Delta X_{t-1}=\left[\begin{array}{c}
\Delta Y_{t-1} \\
\vdots \\
\Delta Y_{t-p+1}
\end{array}\right] \\
U=\left[\begin{array}{lll}
u_{1} & \ldots & u_{T}
\end{array}\right]
\end{gathered}
$$

\subsection{Função de verossimilhança condicional}

Seja $Y_{t}$ um vetor de ordem $(n \times 1)$ seguindo um modelo VEC com vetor de cointegração $\boldsymbol{\beta}$ escrito na forma de decomposição por ondaletas, conforme descrito na Equação 4.5. Vamos supor que cada variável individual $Y_{i t}$ seja $I(1), i=1, \ldots, n$, embora $r<n$ combinações lineares dos elementos de $Y_{t}$ sejam estacionárias. Além disso, $t=1, \ldots, T$, sendo $T$ o número de observações da série $n$ variada. Conforme visto anteriormente na Equação 4.5, esta suposição nos permite escrever $\boldsymbol{\Pi}_{J}=\boldsymbol{\alpha} \mathbf{W}_{J}^{\prime}$, sendo $\boldsymbol{\alpha}$ uma matriz $(n \times r)$ e $\mathbf{W}_{J}^{\prime}$ definida na Equação 4.8. Vamos supor ainda que na Equação 4.5, $u_{t}$ são i.i.d. e seguem uma distribuição $N_{n}\left(\mathbf{0}, \boldsymbol{\Sigma}_{u}\right)$.

Considere uma amostra de $(T+p)$ observações para $\Delta Y_{t}=\left(\Delta Y_{1 t}, \ldots, \Delta Y_{n t}\right)^{\prime}$, com $t=1-p, \ldots, 0,1, \ldots, T$, denotada por

$$
(\underbrace{\Delta Y_{1-p}, \Delta Y_{2-p}, \ldots, \Delta Y_{0}}_{p \text { obs. }}, \underbrace{\Delta Y_{1}, \ldots, \Delta Y_{T}}_{T \text { obs. }}) .
$$

Vamos basear nossa estimação nas últimas $T$ observações das séries de $\Delta Y$, condicionando nas primeiras $p$ observações. O objetivo será construir a verossimilhança condicional

$$
l\left(\boldsymbol{\theta}_{J}\right)=f\left(\Delta Y_{T}, \Delta Y_{T-1}, \ldots, \Delta Y_{1} \mid \Delta Y_{0}, \ldots, \Delta Y_{1-p} ; \boldsymbol{\theta}_{J}\right),
$$

e maximizar com respeito a $\boldsymbol{\theta}_{J}=\left(\boldsymbol{\Pi}_{J}, \boldsymbol{\Gamma}_{1}, \ldots, \boldsymbol{\Gamma}_{p-1}, \boldsymbol{\Sigma}_{u}\right)$.

Condicional às observações até o instante $t-1, \Delta Y_{t}$ é igual a um termo constante acrescido de um termo com distribuição normal, ou seja

$$
\Delta Y_{t} \mid \Delta Y_{t-1}, \ldots, \Delta Y_{1-p}=\underbrace{\boldsymbol{\alpha} \mathbf{W}_{J}^{\prime} Y_{t-1}^{(J)}+\Gamma \Delta X_{t-1}}_{\text {constante }}+u_{t},
$$

$\operatorname{com} u_{t} \sim \mathbf{N}_{n}\left(\mathbf{0}, \boldsymbol{\Sigma}_{u}\right)$.

Pela Equação 4.14, temos ainda que $E\left(\Delta Y_{t} \mid \Delta Y_{t-1}, \ldots, \Delta Y_{1-p}\right)=\boldsymbol{\alpha} \mathbf{W}_{J}^{\prime} Y_{t-1}^{(J)}+\boldsymbol{\Gamma} \Delta X_{t-1}$, enquanto $\operatorname{Var}\left(\Delta Y_{t} \mid \Delta Y_{t-1}, \ldots, \Delta Y_{1-p}\right)=\Sigma_{u}$. Podemos então concluir que 


$$
\Delta Y_{t} \mid \Delta Y_{t-1}, \ldots, \Delta Y_{1-p} \sim \mathbf{N}_{n}\left(\boldsymbol{\alpha} \mathbf{W}_{J}^{\prime} Y_{t-1}^{(J)}+\Gamma \Delta X_{t-1}, \Sigma_{u}\right) .
$$

A densidade da $t$-ésima observação, para $t=1, \ldots, T$, é dada então por

$$
\begin{aligned}
f\left(\Delta Y_{t} \mid \Delta Y_{t-1}, \ldots, \Delta Y_{1-p} ; \boldsymbol{\theta}_{J}\right)= & \frac{1}{(2 \pi)^{\frac{n}{2}}\left|\boldsymbol{\Sigma}_{u}\right|^{\frac{1}{2}}} \cdot \exp \left(-\frac{1}{2}\left(\Delta Y_{t}-\boldsymbol{\alpha} \mathbf{W}_{J}^{\prime} Y_{t-1}^{(J)}-\boldsymbol{\Gamma} \Delta X_{t-1}\right)^{\prime} .\right. \\
& \left.\boldsymbol{\Sigma}_{u}^{-1} \cdot\left(\Delta Y_{t}-\boldsymbol{\alpha} \mathbf{W}_{J}^{\prime} Y_{t-1}^{(J)}-\boldsymbol{\Gamma} \Delta X_{t-1}\right)\right) .
\end{aligned}
$$

Logo, a verossimilhança condicional para a amostra completa $\Delta Y_{T}, \Delta Y_{T-1}, \ldots, \Delta Y_{1} \mid$ $\Delta Y_{0}, \ldots, \Delta Y_{1-p}$ é dada por

$$
f\left(\Delta Y_{T}, \Delta Y_{T-1}, \ldots, \Delta Y_{1} \mid \Delta Y_{0}, \ldots, \Delta Y_{1-p} ; \boldsymbol{\theta}_{J}\right)=\prod_{t=1}^{T} f\left(\Delta Y_{t} \mid \Delta Y_{t-1}, \ldots, \Delta Y_{1-p} ; \boldsymbol{\theta}_{J}\right) .
$$

Estamos interessados no entanto na função log-verossimilhança $\mathscr{L}\left(\boldsymbol{\theta}_{J}\right)$ para o modelo dado na Equação 4.4. Utilizando as Equações 4.16 e 4.17, podemos mostrar que a logverossimilhança, em notação matricial, é dada por

$$
\begin{aligned}
\mathscr{L}\left(\boldsymbol{\theta}_{J}\right)= & -\frac{n T}{2} \log (2 \pi)-\frac{T}{2} \log \left(\left|\boldsymbol{\Sigma}_{u}\right|\right)-\frac{1}{2} \operatorname{tr}\left(\left(\Delta Y-\boldsymbol{\alpha} \mathbf{W}_{J}^{\prime} Y_{-1}^{(J)}-\boldsymbol{\Gamma} \Delta X\right)^{\prime} \cdot \boldsymbol{\Sigma}_{u}^{-1} .\right. \\
& \left.\cdot\left(\Delta Y-\boldsymbol{\alpha} \mathbf{W}_{J}^{\prime} Y_{-1}^{(J)}-\boldsymbol{\Gamma} \Delta X\right)\right) .
\end{aligned}
$$

Nosso objetivo então será escolher $\boldsymbol{\theta}_{J}=\left(\boldsymbol{\Pi}_{J}, \boldsymbol{\Gamma}_{1}, \ldots, \boldsymbol{\Gamma}_{p-1}, \boldsymbol{\Sigma}_{u}\right)$ que maximiza 4.18, sujeito à restrição de que $\boldsymbol{\Pi}_{J}=\boldsymbol{\alpha} \mathbf{W}_{J}^{\prime}$.

\subsection{Estimadores de máxima verossimilhança}

Uma vez obtida a função de verossimilhança para o caso gaussiano, estamos interessados em obter os estimadores de máxima verossimilhança para o modelo VEC com vetor de cointegração variando com o tempo, dado na Equação 4.5. Assim como fizemos para o modelo VEC invariante com o tempo, vamos concentrar a função de verossimilhança com respeito ao termo de correção de erros. Para isso, vamos substituir a matriz de parâmetros $\Gamma$ (de curto prazo) pelo seu estimador de mínimos quadrados, dada uma matriz $\boldsymbol{\Pi}_{J}=\boldsymbol{\alpha} \mathbf{W}_{J}^{\prime}$ fixa:

$$
\hat{\boldsymbol{\Gamma}}\left(\boldsymbol{\alpha} \mathbf{W}_{J}^{\prime}\right)=\left(\Delta Y-\boldsymbol{\alpha} \mathbf{W}_{J}^{\prime} Y_{-1}^{(J)}\right) \Delta X^{\prime}\left(\Delta X \Delta X^{\prime}\right)^{-1}
$$

ou equivalentemente,

$$
\hat{\boldsymbol{\Gamma}}\left(\boldsymbol{\Pi}_{J}\right)=\left(\Delta Y-\Pi_{J} Y_{-1}^{(J)}\right) \Delta X^{\prime}\left(\Delta X \Delta X^{\prime}\right)^{-1}
$$


Substituindo $\hat{\boldsymbol{\Gamma}}\left(\boldsymbol{\Pi}_{J}\right)$ por $\boldsymbol{\Gamma}$ na Equação 4.5, temos que

$$
\begin{aligned}
& \Delta Y=\boldsymbol{\Pi}_{J} Y_{-1}^{(J)}+\hat{\boldsymbol{\Gamma}}\left(\boldsymbol{\alpha} \mathbf{W}_{J}^{\prime}\right) \Delta X+U^{*} \\
& \Delta Y=\boldsymbol{\Pi}_{J} Y_{-1}^{(J)}+\left(\Delta Y-\boldsymbol{\Pi}_{J} Y_{-1}^{(J)}\right) \Delta X^{\prime}\left(\Delta X \Delta X^{\prime}\right)^{-1} \Delta X+U^{*} \\
& \Delta Y=\boldsymbol{\Pi}_{J} Y_{-1}^{(J)}+\Delta Y \Delta X^{\prime}\left(\Delta X \Delta X^{\prime}\right)^{-1} \Delta X-\boldsymbol{\Pi}_{J} Y_{-1}^{(J)} \Delta X^{\prime}\left(\Delta X \Delta X^{\prime}\right)^{-1} \Delta X+U^{*} \\
& \Delta Y=\boldsymbol{\Pi}_{J} Y_{-1}^{(J)} \underbrace{\left[\mathbf{I}_{T}-\Delta X^{\prime}\left(\Delta X \Delta X^{\prime}\right)^{-1} \Delta X\right]}_{M}+\Delta Y \Delta X^{\prime}\left(\Delta X \Delta X^{\prime}\right)^{-1} \Delta X+U^{*} \\
& \Delta Y=\Pi_{J} Y_{-1}^{(J)} \mathbf{M}+\Delta Y \Delta X^{\prime}\left(\Delta X \Delta X^{\prime}\right)^{-1} \Delta X+U^{*} .
\end{aligned}
$$

Rearranjando os termos da igualdade em 4.21, temos que

$$
\begin{aligned}
\Delta Y \underbrace{\left[\mathbf{I}_{T}-\Delta X^{\prime}\left(\Delta X \Delta X^{\prime}\right)^{-1} \Delta X\right]}_{\mathbf{M}} & =\Pi_{J} Y_{-1}^{(J)} \mathbf{M}+U^{*} \\
\underbrace{\Delta Y \mathbf{M}}_{R_{0}} & =\Pi_{J} \underbrace{Y_{-1}^{(J)} \mathbf{M}}_{R_{1}^{(J)}}+U^{*} \\
R_{0} & =\Pi_{J} R_{1}^{(J)}+U^{*} .
\end{aligned}
$$

Assim como feito para o modelo VEC da Equação 2.16, devemos notar que $R_{0}$ é o resíduo de uma regressão multivariada de $\Delta Y$ em $\Delta X$ (Lutkepohl, 2005). Por outro lado, $R_{1}^{(J)}$ é o resíduo de uma regressão multivariada de $Y_{-1}^{(J)}$ em $\Delta X$. Conforme apresentado na Equação 4.9, o termo $Y_{-1}^{(J)}$ incorpora as bases de ondaletas.

Enunciamos a seguir a Proposição 4.1, que nos fornece os estimadores de máxima verossimilhança para os parâmetros do modelo VEC com vetor de cointegração variando com o tempo.

Proposição 4.1. (Estimadores de máxima verossimilhança de um modelo VEC com vetor de cointegração variando com tempo e decomposto via ondaletas)

Sejam as matrizes $\mathbf{M}:=\left[\mathbf{I}_{T}-\Delta X^{\prime}\left(\Delta X \Delta X^{\prime}\right)^{-1} \Delta X\right], R_{0}=\Delta Y \mathbf{M}$ e $R_{1}^{(J)}:=Y_{-1}^{(J)} \mathbf{M}$, apresentadas em 4.22. Definimos as matrizes de covariância amostrais:

$$
\begin{gathered}
\mathbf{S}_{00, T}=\frac{1}{T} R_{0} R_{0}^{\prime}, \\
\mathbf{S}_{11, T}^{(J)}:=\frac{1}{T} R_{1}^{(J)} R_{1}^{(J)^{\prime}}, \\
\mathbf{S}_{10, T}^{(J)}:=\frac{1}{T} R_{1}^{(J)} R_{0}:=\mathbf{S}_{01, T}^{(J)^{\prime}} .
\end{gathered}
$$

Em seguida, ao resolver o problema de autovalores 


$$
\left|\lambda^{(J)} \mathbf{S}_{11, T}^{(J)}-\mathbf{S}_{10, T}^{(J)}\left(\mathbf{S}_{00, T}\right)^{-1} \mathbf{S}_{01, T}^{(J)}\right|=0,
$$

obtemos os autovalores ordenados $\hat{\lambda}_{1}^{(J)} \geq \hat{\lambda}_{2}^{(J)} \geq \ldots \geq \hat{\lambda}_{n}^{(J)}$ e autovetores associados $\hat{\boldsymbol{v}}_{1}, \hat{\boldsymbol{v}}_{2}, \ldots, \hat{\boldsymbol{v}}_{n}$, que satisfazem

$$
\begin{aligned}
\hat{\lambda}_{i}^{(J)} \mathbf{S}_{11, T}^{(J)} \hat{\mathbf{V}}_{i} & =\mathbf{S}_{10, T}^{(J)} \mathbf{S}_{00}^{-1} \mathbf{S}_{01, T}^{(J)} \\
\hat{\mathbf{V}} \mathbf{S}_{11, T}^{(J)} \hat{\mathbf{V}} & =\mathbf{I}_{n}
\end{aligned}
$$

$\operatorname{com} \hat{\mathbf{V}}=\left[\begin{array}{llll}\hat{\boldsymbol{v}}_{1} & \hat{\boldsymbol{v}}_{2} & \ldots & \hat{\boldsymbol{v}}_{n}\end{array}\right]$. Desta forma, a log-verossimilhança do modelo dado na Equação 4.18 é maximizada para

$$
\begin{aligned}
& \hat{\mathbf{W}}_{J}=\left[\begin{array}{llll}
\hat{\boldsymbol{v}}_{1}^{(J)} & \hat{\boldsymbol{v}}_{2}^{(J)} & \ldots & \hat{\boldsymbol{v}}_{r}^{(J)}
\end{array}\right]^{\prime}\left(\mathbf{S}_{11, T}^{(J)}\right)^{-\frac{1}{2}}, \\
& \hat{\boldsymbol{\alpha}}=\Delta Y \mathbf{M} Y_{-1}^{(J)} \hat{\mathbf{W}}_{J}\left(\hat{\mathbf{W}}_{J}^{\prime} Y_{-1}^{(J)} \mathbf{M} Y_{-1}^{(J)^{\prime}} \hat{\mathbf{W}}_{J}\right)^{-1}=S_{01, T}^{(J)} \hat{\mathbf{W}}_{J}\left(\hat{\mathbf{W}}_{J}^{\prime} \mathbf{S}_{11, T}^{(J)} \hat{\mathbf{W}}_{J}\right)^{-1} \\
& \hat{\boldsymbol{\Gamma}}=\left(\Delta Y-\hat{\boldsymbol{\alpha}} \hat{\mathbf{W}}_{J}^{\prime} Y_{-1}^{(J)}\right) \Delta X^{\prime}\left(\Delta X \Delta X^{\prime}\right)^{-1}, \\
& \hat{\boldsymbol{\Sigma}}_{u}=\frac{1}{T}\left(\Delta Y-\hat{\boldsymbol{\alpha}} \hat{\mathbf{W}}_{J}^{\prime} Y_{-1}^{(J)}-\hat{\boldsymbol{\Gamma}} \Delta X\right)\left(\Delta Y-\hat{\boldsymbol{\alpha}} \hat{\mathbf{W}}_{J}^{\prime} Y_{-1}^{(J)}-\hat{\boldsymbol{\Gamma}} \Delta X\right)^{\prime} .
\end{aligned}
$$

Além disso, o máximo de 4.18 é atingido em

$$
\max \mathscr{L}\left(\boldsymbol{\theta}_{J}\right)=-\frac{n T}{2} \ln 2 \pi-\frac{T}{2}\left[\ln \left|\mathbf{S}_{00}\right|+\sum_{i=1}^{r} \ln \left(1-\lambda_{i}^{J}\right)\right]-\frac{n T}{2} .
$$

\section{Demonstração:}

Para $\boldsymbol{\alpha}$ e $\mathbf{W}_{J}^{\prime}$, o máximo de $\mathscr{L}(\boldsymbol{\theta})$ em 4.18 é atingido para

$$
\hat{\boldsymbol{\Gamma}}\left(\boldsymbol{\alpha} \mathbf{W}_{J}^{\prime}\right)=\left(\Delta Y-\boldsymbol{\alpha} \mathbf{W}_{J}^{\prime} Y_{-1}^{(J)}\right) \Delta X^{\prime}\left(\Delta X \Delta X^{\prime}\right)^{-1}
$$

e

$$
\hat{\mathbf{\Sigma}}_{u}\left(\boldsymbol{\alpha} \mathbf{W}_{J}^{\prime}\right)=\frac{1}{T}\left(\Delta Y \mathbf{M}-\boldsymbol{\alpha} \mathbf{W}_{J}^{\prime} Y_{-1}^{(J)} \mathbf{M}\right)\left(\Delta Y \mathbf{M}-\boldsymbol{\alpha} \mathbf{W}_{J}^{\prime} Y_{-1}^{(J)} \mathbf{M}\right)^{\prime}
$$

Substituindo $\boldsymbol{\Gamma}$ de 4.18 por $\hat{\boldsymbol{\Gamma}}\left(\boldsymbol{\alpha} \mathbf{W}_{J}^{\prime}\right)$ de 4.33 , temos a log-verossimilhança concentrada 


$$
\begin{aligned}
& \mathscr{L}_{c}(\boldsymbol{\theta})=-\frac{n T}{2} \ln (2 \pi)-\frac{T}{2} \ln \left(\left|\boldsymbol{\Sigma}_{u}\right|\right)-\frac{1}{2} \operatorname{tr}\left[\left(\Delta Y-\boldsymbol{\alpha} \mathbf{W}_{J}^{\prime} Y_{-1}^{(J)}-\hat{\boldsymbol{\Gamma}}\left(\boldsymbol{\alpha} \mathbf{W}_{J}^{\prime}\right) \Delta X\right)^{\prime} \cdot \boldsymbol{\Sigma}_{u}^{-1}\right. \\
& \left.\cdot\left(\Delta Y-\boldsymbol{\alpha} \mathbf{W}_{J}^{\prime} Y_{-1}^{(J)}-\hat{\Gamma}\left(\boldsymbol{\alpha} \mathbf{W}_{J}^{\prime}\right) \Delta X\right)\right], \\
& =-\frac{n T}{2} \ln (2 \pi)-\frac{T}{2} \ln \left(\left|\boldsymbol{\Sigma}_{u}\right|\right)-\frac{1}{2} \operatorname{tr}\left[\left(\Delta Y-\boldsymbol{\alpha} \mathbf{W}_{J}^{\prime} Y_{-1}^{(J)}-\left(\Delta Y-\boldsymbol{\alpha} \mathbf{W}_{J^{\prime}}^{\prime} Y_{-1}^{(J)}\right) \Delta X^{\prime}\left(\Delta X \Delta X^{\prime}\right)^{-1} \Delta X\right)^{\prime} .\right. \\
& \left.\cdot \boldsymbol{\Sigma}_{u}^{-1} \cdot\left(\Delta Y-\boldsymbol{\alpha} \mathbf{W}_{J}^{\prime} Y_{-1}^{(J)}-\left(\Delta Y-\boldsymbol{\alpha} \mathbf{W}_{J}^{\prime} Y_{-1}^{(J)}\right) \Delta X^{\prime}\left(\Delta X \Delta X^{\prime}\right)^{-1} \Delta X\right)\right], \\
& =-\frac{n T}{2} \ln (2 \pi)-\frac{T}{2} \ln \left(\left|\boldsymbol{\Sigma}_{u}\right|\right)-\frac{1}{2} \operatorname{tr}\left[(\left(\Delta Y-\boldsymbol{\alpha} \mathbf{W}_{J}^{\prime} Y_{-1}^{(J)}\right) \underbrace{\left(\mathbf{I}_{T}-\Delta X^{\prime}\left(\Delta X \Delta X^{\prime}\right)^{-1} \Delta X\right)}_{\mathbf{M}})^{\prime} .\right. \\
& \text { - } \left.\boldsymbol{\Sigma}_{u}^{-1} \cdot(\left(\Delta Y-\boldsymbol{\alpha} \mathbf{W}_{J}^{\prime} Y_{-1}^{(J)}\right) \underbrace{\left(\mathbf{I}_{T}-\Delta X^{\prime}\left(\Delta X \Delta X^{\prime}\right)^{-1} \Delta X\right)}_{\mathbf{M}})\right] \\
& =-\frac{n T}{2} \ln (2 \pi)-\frac{T}{2} \ln \left(\left|\boldsymbol{\Sigma}_{u}\right|\right)-\frac{1}{2} \operatorname{tr}\left[\left(\Delta Y \mathbf{M}-\boldsymbol{\alpha} \mathbf{W}_{J}^{\prime} Y_{-1}^{(J)} \mathbf{M}\right)^{\prime} \cdot \boldsymbol{\Sigma}_{u}^{-1} \cdot\left(\Delta Y \mathbf{M}-\boldsymbol{\alpha} \mathbf{W}_{J}^{\prime} Y_{-1}^{(J)} \mathbf{M}\right)\right] .
\end{aligned}
$$

A seguir, vamos maximizar 4.35 com relação à $\boldsymbol{\Sigma}_{u}\left(\boldsymbol{\alpha} \mathbf{W}_{J}^{\prime}\right)$. Substituindo $\boldsymbol{\Sigma}_{u}\left(\boldsymbol{\alpha} \mathbf{W}_{J}^{\prime}\right)$ de 4.35 por $\hat{\boldsymbol{\Sigma}}_{u}\left(\boldsymbol{\alpha} \mathbf{W}_{J}^{\prime}\right)$ de 4.34 , temos que

$$
\begin{aligned}
\mathscr{L}_{c}^{\prime}(\boldsymbol{\theta})= & -\frac{n T}{2} \ln (2 \pi)-\frac{T}{2} \ln \left(\left|\hat{\boldsymbol{\Sigma}}_{u}\left(\boldsymbol{\alpha} \mathbf{W}_{J}^{\prime}\right)\right|\right)- \\
& -\frac{1}{2} \operatorname{tr}[\underbrace{\left(\Delta Y \mathbf{M}-\boldsymbol{\alpha} \mathbf{W}_{J}^{\prime} Y_{-1}^{(J)} \mathbf{M}\right)^{\prime} \cdot \hat{\boldsymbol{\Sigma}}_{u}^{-1}\left(\boldsymbol{\alpha} \mathbf{W}_{J}^{\prime}\right) \cdot\left(\Delta Y \mathbf{M}-\boldsymbol{\alpha} \mathbf{W}_{J}^{\prime} Y_{-1}^{(J)} \mathbf{M}\right)}_{T I_{n}}] .
\end{aligned}
$$

Note ainda que o termo que está no interior de $\operatorname{tr}[\cdot]$ na Equação 4.36 é igual ao produto de $T$ por uma matriz identidade de ordem $n\left(T I_{n}\right)$, pois

$$
\begin{aligned}
& \left(\Delta Y \mathbf{M}-\boldsymbol{\alpha} \mathbf{W}_{J}^{\prime} Y_{-1}^{(J)} \mathbf{M}\right)^{\prime} \cdot \hat{\boldsymbol{\Sigma}}_{u}^{-1}\left(\boldsymbol{\alpha} \mathbf{W}_{J}^{\prime}\right) \cdot\left(\Delta Y \mathbf{M}-\boldsymbol{\alpha} \mathbf{W}_{J}^{\prime} Y_{-1}^{(J)} \mathbf{M}\right)= \\
& \left(\Delta Y \mathbf{M}-\boldsymbol{\alpha} \mathbf{W}_{J}^{\prime} Y_{-1}^{(J)} \mathbf{M}\right)^{\prime} \cdot\left(\frac{1}{T}\left(\Delta Y \mathbf{M}-\boldsymbol{\alpha} \mathbf{W}_{J}^{\prime} Y_{-1}^{(J)} \mathbf{M}\right)\left(\Delta Y \mathbf{M}-\boldsymbol{\alpha} \mathbf{W}_{J}^{\prime} Y_{-1}^{(J)} \mathbf{M}\right)^{\prime}\right)^{-1} \cdot\left(\Delta Y \mathbf{M}-\boldsymbol{\alpha} \mathbf{W}_{J}^{\prime} Y_{-1}^{(J)} \mathbf{M}\right)= \\
& T \underbrace{\left(\Delta Y \mathbf{M}-\boldsymbol{\alpha} \mathbf{W}_{J}^{\prime} Y_{-1}^{(J)} \mathbf{M}\right)^{\prime} \cdot\left(\Delta Y \mathbf{M}-\boldsymbol{\alpha} \mathbf{W}_{J}^{\prime} Y_{-1}^{(J)} \mathbf{M}\right)^{\prime}}_{I_{n}} \cdot \underbrace{\left(\Delta Y \mathbf{M}-\boldsymbol{\alpha} \mathbf{W}_{J}^{\prime} Y_{-1}^{(J)} \mathbf{M}\right)^{-1} \cdot\left(\Delta Y \mathbf{M}-\boldsymbol{\alpha} \mathbf{W}_{J}^{\prime} Y_{-1}^{(J)} \mathbf{M}\right)}_{I_{n}}=
\end{aligned}
$$

$T I_{n}$.

Consequentemente, usando 4.37, a Equação 4.36 pode ser reescrita como

$$
\begin{aligned}
\mathscr{L}_{c}^{\prime}(\boldsymbol{\theta}) & =-\frac{n T}{2} \ln (2 \pi)-\frac{T}{2} \ln \left(\left|\frac{1}{T}\left(\Delta Y \mathbf{M}-\boldsymbol{\alpha} \mathbf{W}_{J}^{\prime} Y_{-1}^{(J)} \mathbf{M}\right)\left(\Delta Y \mathbf{M}-\boldsymbol{\alpha} \mathbf{W}_{J}^{\prime} Y_{-1}^{(J)} \mathbf{M}\right)^{\prime}\right|\right)-\frac{1}{2} \operatorname{tr}\left(T I_{n}\right) \\
& =\underbrace{-\frac{n T}{2} \ln (2 \pi)-\frac{T n}{2}}_{\text {escalar }}-\frac{T}{2} \ln \left(\left|\frac{1}{T}\left(\Delta Y \mathbf{M}-\boldsymbol{\alpha} \mathbf{W}_{J}^{\prime} Y_{-1}^{(J)} \mathbf{M}\right)\left(\Delta Y \mathbf{M}-\boldsymbol{\alpha} \mathbf{W}_{J}^{\prime} Y_{-1}^{(J)} \mathbf{M}\right)^{\prime}\right|\right) .
\end{aligned}
$$


Desconsiderando a soma de termos escalares em 4.38, para maximizar $\mathscr{L}_{c}^{\prime}(\boldsymbol{\theta})$ temos que maximizar

$$
-\frac{T}{2} \ln \left(\left|\frac{1}{T}\left(\Delta Y \mathbf{M}-\boldsymbol{\alpha} \mathbf{W}_{J}^{\prime} Y_{-1}^{(J)} \mathbf{M}\right)\left(\Delta Y \mathbf{M}-\boldsymbol{\alpha} \mathbf{W}_{J}^{\prime} Y_{-1}^{(J)} \mathbf{M}\right)^{\prime}\right|\right)
$$

ou equivalentemente, minimizar

$$
\left|\frac{1}{T}\left(\Delta Y \mathbf{M}-\boldsymbol{\alpha} \mathbf{W}_{J}^{\prime} Y_{-1}^{(J)} \mathbf{M}\right)\left(\Delta Y \mathbf{M}-\boldsymbol{\alpha} \mathbf{W}_{J}^{\prime} Y_{-1}^{(J)} \mathbf{M}\right)^{\prime}\right|
$$

com respeito a $\boldsymbol{\alpha}$ e $\mathbf{W}_{J}$.

Para demonstrar este resultado, vamos aplicar a Proposição A.1 em 4.40, tomando $Y=\Delta Y \mathbf{M}, X=Y_{-1}^{(J)} \mathbf{M}, A=\boldsymbol{\alpha}$ e $B=\mathbf{W}_{J}^{\prime}$. Além disso, vamos considerar as matrizes de covariância amostrais $\mathbf{S}_{i, j}$ definidas em $4.23,4.24$ e 4.25. Neste caso, de A.1, temos que

$$
\begin{aligned}
\hat{\mathbf{W}}_{J}^{\prime} & =\left[\begin{array}{llll}
\hat{\boldsymbol{v}}_{1} & \hat{\boldsymbol{v}}_{2} & \ldots & \hat{\boldsymbol{v}}_{r}
\end{array}\right]^{\prime}\left(X X^{\prime}\right)^{-\frac{1}{2}} \\
& =\left[\begin{array}{llll}
\hat{\boldsymbol{v}}_{1} & \hat{\boldsymbol{v}}_{2} & \ldots & \hat{\boldsymbol{v}}_{r}
\end{array}\right]^{\prime}\left(\left(Y_{-1}^{(J)} \mathbf{M}\right)\left(Y_{-1}^{(J)} \mathbf{M}\right)^{\prime}\right)^{-\frac{1}{2}} \\
& =\left[\begin{array}{llll}
\hat{\boldsymbol{v}}_{1} & \hat{\boldsymbol{v}}_{2} & \ldots & \hat{\boldsymbol{v}}_{r}
\end{array}\right]^{\prime}\left(R_{1}^{(J)} R_{1}^{(J)^{\prime}}\right)^{-\frac{1}{2}} \\
& =\left[\begin{array}{llll}
\hat{\boldsymbol{v}}_{1} & \hat{\boldsymbol{v}}_{2} & \ldots & \hat{\boldsymbol{v}}_{r}
\end{array}\right]^{\prime}\left(\mathbf{S}_{11, T}^{(J)}\right)^{-\frac{1}{2}}
\end{aligned}
$$

Além disso, ainda pelo resultado apresentado em A.1, temos que

$$
\begin{aligned}
\hat{\boldsymbol{\alpha}} & =Y X^{\prime} \hat{B}^{\prime}\left(\hat{B} X X^{\prime} \hat{B}^{\prime}\right)^{-1} \\
& =(\Delta Y \mathbf{M})\left(Y_{-1}^{(J)} \mathbf{M}\right)^{\prime} \mathbf{W}_{J}\left(\mathbf{W}_{J}^{\prime}\left(Y_{-1}^{(J)} \mathbf{M}\right)\left(Y_{-1}^{(J)} \mathbf{M}\right)^{\prime} \mathbf{W}_{J}\right)^{-1} \\
& =R_{0} R_{1}^{(J)^{\prime}} \mathbf{W}_{J}\left(\mathbf{W}_{J}^{\prime} R_{1}^{(J)^{\prime}} R_{1}^{(J)^{\prime}} \mathbf{W}_{J}^{\prime}\right)^{-1} \\
& =\mathbf{S}_{01, T}^{(J)} \mathbf{W}_{J}\left(\mathbf{W}_{J}^{\prime} \mathbf{S}_{11, T}^{(J)} \mathbf{W}_{J}\right)^{-1} .
\end{aligned}
$$

Estimamos em seguida os demais parâmetros do modelo, substituindo $\hat{\mathbf{W}}_{J}$ e $\hat{\boldsymbol{\alpha}}$ das Equações 4.41 e 4.42 nas Equações 4.33 e 4.34. Desta forma, temos:

e

$$
\hat{\boldsymbol{\Gamma}}=\left(\Delta Y-\hat{\boldsymbol{\alpha}} \hat{\mathbf{W}}_{J}^{\prime} Y_{-1}\right) \Delta X^{\prime}\left(\Delta X \Delta X^{\prime}\right)^{-1}
$$

$$
\hat{\boldsymbol{\Sigma}}_{u}=\frac{1}{T}\left(\Delta Y \mathbf{M}-\hat{\boldsymbol{\alpha}} \hat{\mathbf{W}}_{J}^{\prime} Y_{-1}^{(J)} \mathbf{M}\right)\left(\Delta Y \mathbf{M}-\hat{\boldsymbol{\alpha}} \hat{\mathbf{W}}_{J}^{\prime} Y_{-1} \mathbf{M}\right)^{\prime} .
$$

Substituindo $\mathbf{M}=\mathbf{I}_{T}-\Delta X^{\prime}\left(\Delta X \Delta X^{\prime}\right)^{-1} \Delta X$ no termo $\left(\Delta Y \mathbf{M}-\hat{\boldsymbol{\alpha}} \hat{\mathbf{W}}_{J}^{\prime} Y_{-1} \mathbf{M}\right)$ da Equação 4.44, obtemos: 


$$
\begin{aligned}
\left(\Delta Y \mathbf{M}-\hat{\boldsymbol{\alpha}} \hat{\mathbf{W}}_{J}^{\prime} Y_{-1}^{(J)} \mathbf{M}\right) & =\Delta Y\left(\mathbf{I}_{T}-\Delta X^{\prime}\left(\Delta X \Delta X^{\prime}\right)^{-1} \Delta X\right)-\hat{\boldsymbol{\alpha}} \hat{\mathbf{W}}_{J}^{\prime} Y_{-1}^{(J)}\left(\mathbf{I}_{T}-\Delta X^{\prime}\left(\Delta X \Delta X^{\prime}\right)^{-1} \Delta X\right) \\
& =\Delta Y-\hat{\boldsymbol{\alpha}} \hat{\mathbf{W}}_{J}^{\prime} Y_{-1}^{(J)}-\underbrace{\left(\Delta Y-\hat{\boldsymbol{\alpha}} \hat{\mathbf{W}}_{J}^{\prime} Y_{-1}^{(J)}\right) \Delta X^{\prime}\left(\Delta X \Delta X^{\prime}\right)^{-1}}_{\hat{\boldsymbol{\Gamma}}} \Delta X \\
& =\Delta Y-\hat{\boldsymbol{\alpha}} \hat{\mathbf{W}}_{J}^{\prime} Y_{-1}^{(J)}-\hat{\boldsymbol{\Gamma}} \Delta X
\end{aligned}
$$

Desta forma, substituindo 4.45 em 4.44, temos:

$$
\hat{\boldsymbol{\Sigma}}_{u}=\frac{1}{T}\left(\Delta Y-\hat{\boldsymbol{\alpha}} \hat{\mathbf{W}}_{J}^{\prime} Y_{-1}^{(J)}-\hat{\boldsymbol{\Gamma}} \Delta X\right)\left(\Delta Y-\hat{\boldsymbol{\alpha}} \hat{\mathbf{W}}_{J}^{\prime} Y_{-1}^{(J)}-\hat{\boldsymbol{\Gamma}} \Delta X\right)^{\prime}
$$

Pelo Apêndice A.1, o mínimo da expressão 4.40 é dado por:

$$
\min _{\boldsymbol{\alpha}, \boldsymbol{\beta}}\left|\frac{1}{T}\left(\Delta Y \mathbf{M}-\boldsymbol{\alpha} \hat{\mathbf{W}}_{J}^{\prime} Y_{-1}^{(J)} \mathbf{M}\right)\left(\Delta Y \mathbf{M}-\boldsymbol{\alpha} \hat{\mathbf{W}}_{J}^{\prime} Y_{-1}^{(J)} \mathbf{M}\right)^{\prime}\right|=\left|\frac{1}{T}(\Delta Y \mathbf{M})(\Delta Y \mathbf{M})^{\prime}\right| \prod_{i=1}^{r} \ln \left(1-\lambda_{i}\right),
$$

que é equivalente a maximizar 4.39.

Substituindo 4.47 em 4.38, temos que o máximo da log-verossimilhança é atingido em:

$$
\begin{aligned}
\mathscr{L}_{c}^{\prime}(\boldsymbol{\theta}) & =-\frac{n T}{2} \ln (2 \pi)-\frac{n T}{2}-\frac{T}{2} \ln \left(\left|\frac{1}{T}(\Delta Y \mathbf{M})(\Delta Y \mathbf{M})^{\prime}\right| \prod_{i=1}^{r} \ln \left(1-\lambda_{i}^{J}\right)\right) \\
& =-\frac{n T}{2} \ln (2 \pi)-\frac{n T}{2}-\frac{T}{2} \ln \left(\left|\mathbf{S}_{00}\right| \prod_{i=1}^{r} \ln \left(1-\lambda_{i}^{J}\right)\right) \\
& =-\frac{T}{2} \sum_{i=1}^{r} \ln \left(1-\lambda_{i}^{J}\right)-\frac{T}{2} \ln \left(\left|\mathbf{S}_{00}\right|\right)-\frac{n T}{2} \ln (2 \pi)-\frac{n T}{2} \\
& =-\frac{n T}{2} \ln 2 \pi-\frac{T}{2}\left[\ln \left|\mathbf{S}_{00}\right|+\sum_{i=1}^{r} \ln \left(1-\lambda_{i}^{J}\right)\right]-\frac{n T}{2},
\end{aligned}
$$

conforme queríamos demonstrar em 4.32.

\subsection{Construindo os estimadores de máxima verossi- milhança dos parâmetros}

Para o cálculo dos estimadores de máxima verossimilhança, utilizamos os passos empregados no artigo seminal de Johansen (1988), aplicando as adaptações necessárias para o caso com ondaletas. Seguimos de perto os desdobramentos de Lutkepohl (2005), Morettin (2011), além dos métodos empregados por Bierens e Martins (2010).

Construímos inicialmente as seguintes matrizes: 


$$
\begin{gathered}
\mathbf{S}_{00, T}=\frac{1}{T} \sum_{t=1}^{T} \Delta Y_{t}\left(\Delta Y_{t}\right)^{\prime}-\widehat{\mathbf{\Sigma}}_{X \Delta Y}^{\prime}\left(\widehat{\boldsymbol{\Sigma}}_{X X}\right)^{-1} \widehat{\mathbf{\Sigma}}_{X \Delta Y} \\
\mathbf{S}_{11, T}^{(J)}=\frac{1}{T} \sum_{t=1}^{T} Y_{t-1}^{(J)}\left(Y_{t-1}^{(J)}\right)^{\prime}-\widehat{\mathbf{\Sigma}}_{X Y(J)}^{\prime}\left(\widehat{\boldsymbol{\Sigma}}_{X X}\right)^{-1} \widehat{\mathbf{\Sigma}}_{X Y^{(J)}}, \\
\mathbf{S}_{01, T}^{(J)}=\frac{1}{T} \sum_{t=1}^{T} \Delta Y_{t}\left(Y_{t-1}^{(J)}\right)^{\prime}-\widehat{\mathbf{\Sigma}}_{X \Delta Y}^{\prime}\left(\widehat{\mathbf{\Sigma}}_{X X}\right)^{-1} \widehat{\mathbf{\Sigma}}_{X Y^{(J)}}, \\
\mathbf{S}_{10, T}^{(J)}=\left(\mathbf{S}_{01, T}^{(J)}\right)^{\prime}
\end{gathered}
$$

com:

$$
\begin{gathered}
\widehat{\mathbf{\Sigma}}_{X X}=\frac{1}{T} \sum_{t=1}^{T} \Delta X_{t}\left(\Delta X_{t}\right)^{\prime} \\
\widehat{\mathbf{\Sigma}}_{X \Delta Y}=\frac{1}{T} \sum_{t=1}^{T} \Delta X_{t}\left(\Delta Y_{t}\right)^{\prime}, \\
\widehat{\mathbf{\Sigma}}_{X Y^{(J)}}=\frac{1}{T} \sum_{t=1}^{T} \Delta X_{t}\left(Y_{t-1}^{(J)}\right)^{\prime}
\end{gathered}
$$

Portanto, seguindo a metodologia de Johansen (1988), os estimadores de máxima verossimilhança dos parâmetros do modelo, bem como o teste de razão de verossimilhanças, são obtidos ao resolvermos o problema de autovalores

$$
\left|\lambda \mathbf{S}_{11, T}^{(J)}-\mathbf{S}_{10, T}^{(J)}\left(\mathbf{S}_{00, T}\right)^{-1} \mathbf{S}_{01, T}^{(J)}\right|=0,
$$

ou equivalentemente (Johansen, 1995),

$$
\left|\lambda \mathbf{I}-\left(\mathbf{S}_{11, T}^{(J)}\right)^{-\frac{1}{2}} \mathbf{S}_{10, T}^{(J)}\left(\mathbf{S}_{00, T}\right)^{-1} \mathbf{S}_{01, T}^{(J)}\left(\mathbf{S}_{11, T}^{(J)}\right)^{-\frac{1}{2}}\right|=0 .
$$

Sejam $\hat{\lambda}_{1}^{(J)} \geq \hat{\lambda}_{2}^{(J)} \geq \ldots \geq \hat{\lambda}_{n}^{(J)}$ os autovalores obtidos ao resolvermos a Equação 4.4, com respectivos autovetores ortonormais $\hat{\boldsymbol{v}}_{1}^{(J)}, \hat{\boldsymbol{v}}_{2}^{(J)}, \ldots, \hat{\boldsymbol{v}}_{n}^{(J)}$. O estimador de máxima verossimilhança de $\boldsymbol{W}_{J}$ é dado por

$$
\hat{\mathbf{W}}_{J}=\left[\begin{array}{llll}
\hat{\boldsymbol{v}}_{1}^{(J)} & \hat{\boldsymbol{v}}_{2}^{(J)} & \ldots & \hat{\boldsymbol{v}}_{r}^{(J)}
\end{array}\right]^{\prime}\left(\mathbf{S}_{11, T}^{(J)}\right)^{-\frac{1}{2}}
$$

com $0<r<n$ (Lutkepohl, 2005).

Assim, ao resolvermos o problema de autovalores na Equação 4.50, encontramos o estimador de máxima verossimilhança de $\boldsymbol{W}_{J}$, que representa os estimadores de máxima verossimilhança de $\mathbf{c}_{\mathbf{0}, \mathbf{0}}, \mathbf{d}_{0,0}, \mathbf{d}_{1,0}, \ldots \mathbf{d}_{J, 2^{J}-1}$, conforme mostrado na Equação 4.8. 
Com base nestes estimadores, podemos obter o estimador de máxima verossimilhança de $\boldsymbol{\beta}_{t}$, tomando $n=2, r=1$ e utilizando a decomposição por ondaletas, para $J=0$ inicialmente:

$$
\hat{\boldsymbol{\beta}}(t)=\left[\begin{array}{l}
\hat{\beta}_{1}(t) \\
\hat{\beta}_{2}(t)
\end{array}\right]=\left[\begin{array}{l}
\hat{c}_{0,0}^{(1,1)} \phi_{0,0}(t)+\hat{d}_{0,0}^{(1,1)} \psi_{0,0}(t) \\
\hat{c}_{0,0}^{(2,1)} \phi_{0,0}(t)+\hat{d}_{0,0}^{(2,1)} \psi_{0,0}(t)
\end{array}\right] .
$$

Por outro lado, se tomarmos $n=2, r=1$ e parâmetro de ondaleta $J=1$, o estimador de máxima verossimilhança de $\beta_{t}$ será obtido por

$$
\hat{\boldsymbol{\beta}}(t)=\left[\begin{array}{l}
\hat{\beta}_{1}(t) \\
\hat{\beta}_{2}(t)
\end{array}\right]=\left[\begin{array}{l}
\hat{c}_{0,0}^{(1,1)} \phi_{0,0}(t)+\hat{d}_{0,0}^{(1,1)} \psi_{0,0}(t)+\hat{d}_{1,0}^{(1,1)} \psi_{1,0}(t)+\hat{d}_{1,1}^{(1,1)} \psi_{1,1}(t) \\
\hat{c}_{0,0}^{(2,1)} \phi_{0,0}(t)+\hat{d}_{0,0}^{(2,1)} \psi_{0,0}(t)+\hat{d}_{1,0}^{(2,1)} \psi_{1,0}(t)+\hat{d}_{1,1}^{(2,1)} \psi_{1,1}(t)
\end{array}\right] .
$$

\subsubsection{Estimadores normalizados}

Um modo de obter a unicidade do estimador de máxima verossimilhança (identificabilidade) está condicionada à suposição de que $\beta_{t}$ tem a forma (Lutkepohl, 2005)

$$
\boldsymbol{\beta}^{*}(t)=\left[\begin{array}{c}
\boldsymbol{\beta}_{1}(t) \boldsymbol{\beta}_{1}^{-1}(t) \\
\boldsymbol{\beta}_{2}(t) \boldsymbol{\beta}_{1}^{-1}(t)
\end{array}\right]=\left[\begin{array}{c}
I_{r} \\
\boldsymbol{\beta}_{n-r}^{*}(t)
\end{array}\right] .
$$

Diremos que se $\boldsymbol{\beta}_{t}$ estiver na forma da Equação 4.54, ele estará normalizado. Caso não tenha esta forma, é possível ordená-lo para garantir que suas primeiras linhas sejam linearmente independentes. Nesta normalização, temos que $\boldsymbol{\beta}_{n-r}^{*}(t)$ é uma matriz de dimensões $(n-r) \times r$ (Lutkepohl, 2005).

Para apresentar a estimação de $\boldsymbol{\beta}_{t}$ normalizado, consideramos exemplos em que $n=2$, $r=1$ e apenas uma mudança para $\boldsymbol{\beta}^{*}(t)$ ao longo do tempo. $\operatorname{Logo}, \boldsymbol{\beta}^{*}(t):=\boldsymbol{\beta}_{t}^{*}$ poderá ser escrito da seguinte maneira:

$$
\boldsymbol{\beta}_{t}^{*}=\left[\begin{array}{c}
\boldsymbol{\beta}_{1}^{*}(t) \\
\boldsymbol{\beta}_{2}^{*}(t)
\end{array}\right]=\left[\begin{array}{c}
I_{1} \\
\boldsymbol{\beta}_{n-r}^{*}(t)
\end{array}\right]=\left[\begin{array}{c}
1 \\
\boldsymbol{\beta}_{n-r}^{*}(t)
\end{array}\right]=\left[\begin{array}{cccccc}
1 & \ldots & 1 & 1 & \ldots & 1 \\
\beta_{21}^{*} & \ldots & \beta_{21}^{*} & \beta_{22}^{*} & \ldots & \beta_{22}^{*}
\end{array}\right] .
$$

Note que os termos normalizados $\boldsymbol{\beta}_{2 t}^{*}$ da Equação 4.55 podem ser representado por

$$
\boldsymbol{\beta}_{2 t}^{*}=\left\{\begin{array}{ll}
\beta_{21}^{*} & , \text { se } 1 \leq t \leq \frac{T}{2} \\
\beta_{22}^{*} & , \text { se } \frac{T}{2}<t \leq T
\end{array} .\right.
$$

Utilizando a Equação 4.52 e supondo que $\hat{\boldsymbol{\beta}}_{t}$ é escrito como na Equação 4.55, podemos encontrar os valores normalizados de $\hat{c}_{0,0}^{*(1,1)}, \hat{c}_{0,0}^{*(2,1)}, \hat{d}_{0,0}^{*(1,1)}$ e $\hat{d}_{0,0}^{*(2,1)}$ ao resolvermos conjuntamente os sistemas: 


$$
\left\{\begin{array} { l } 
{ c _ { 0 , 0 } ^ { * ( 1 , 1 ) } + d _ { 0 , 0 } ^ { * ( 1 , 1 ) } = \hat { \beta } _ { 1 1 } ^ { * } = 1 } \\
{ c _ { 0 , 0 } ^ { * ( 1 , 1 ) } - d _ { 0 , 0 } ^ { * ( 1 , 1 ) } = \hat { \beta } _ { 1 2 } ^ { * } = 1 }
\end{array} \text { e } \left\{\begin{array}{l}
c_{0,0}^{*(2,1)}+d_{0,0}^{*(2,1)}=\hat{\beta}_{21}^{*} \\
c_{0,0}^{*(2,1)}-d_{0,0}^{*(2,1)}=\hat{\beta}_{22}^{*}
\end{array}\right.\right.
$$

Resolvendo o primeiro sistema da Equação 4.52, verificamos que

$$
\hat{c}_{0,0}^{*(1,1)}=1 \text { e } \hat{d}_{0,0}^{*(1,1)}=0
$$

para todo $t$ tal que $1 \leq t<T$.

Da mesma forma, ao resolvermos o segundo sistema da Equação 4.52, verificamos que

$$
\begin{aligned}
& \hat{c}_{0,0}^{*(2,1)}=\frac{\hat{\beta}_{21}^{*}+\hat{\beta}_{22}^{*}}{2}, \\
& \hat{d}_{0,0}^{*(2,1)}=\frac{\hat{\beta}_{21}^{*}-\hat{\beta}_{22}^{*}}{2} .
\end{aligned}
$$

Note que a expressão 4.59 é média de $\hat{\beta}_{21}^{*}$ e $\hat{\beta}_{22}^{*}$ (aproximação), enquanto 4.60 consiste na diferença entre $\hat{\beta}_{21}^{*}$ e $\hat{\beta}_{22}^{*}$ (detalhe). Isto faz sentido, já que consideramos apenas uma mudança para o vetor de cointegração ao longo do tempo, a partir de dois regimes distintos. Note também que estamos adotando a base Haar de ondaletas.

De uma maneira mais geral, considerando $T$ mudanças ao longo do tempo e parâmetro wavelet $J=0$, podemos mostrar que $\hat{c}_{0,0}^{*(1,1)}=1$ e $\hat{d}_{0,0}^{*(1,1)}=0$, para todo $t$ tal que $1 \leq t<T$, assim como na Equação 4.58. Além disso,

$$
\begin{gathered}
\hat{c}_{0,0}^{*(2,1)}=\frac{1}{T} \sum_{i=1}^{T} \hat{\beta}_{2 i}=\overline{\hat{\beta}_{2 t}}, \\
\hat{d}_{0,0}^{*(2,1)}=\frac{1}{T}\left(\sum_{i=1}^{\frac{T}{2}} \hat{\beta}_{2 i}-\sum_{i=\frac{T}{2}+1}^{T} \hat{\beta}_{2 i}\right) .
\end{gathered}
$$

Logo, das Equações 4.58, 4.59 e 4.60 (ou 4.58, 4.61 e 4.62), temos que estimador de máxima verossimilhança de $\mathbf{W}_{J}$ normalizado é dado por

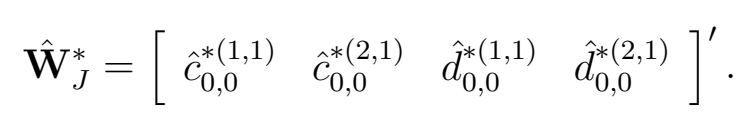

Se for considerado um caso ainda mais geral, conforme o apresentado na Equação 4.53, com $n=2, r=1$ e parâmetro wavelet $J=1$, é possível mostrar que

$$
\hat{c}_{0,0}^{*(1,1)}=1, \hat{d}_{0,0}^{*(1,1)}=0, \hat{d}_{1,0}^{*(1,1)}=0 \text { e } \hat{d}_{1,1}^{*(1,1)}=0 .
$$

Além disso, os termos $c_{0,0}^{*(2,1)}$ e $d_{0,0}^{*(2,1)}$ são estimados respectivamente pelas Equações 4.61 e 4.62. Os termos $d_{1,0}^{*(2,1)}$ e $d_{1,1}^{*(2,1)}$ por sua vez, são estimados pelas Equações 4.65 e 4.66 a seguir: 


$$
\begin{gathered}
\hat{d}_{1,0}^{*(2,1)}=\frac{1}{T}\left(2^{\frac{3}{2}} \sum_{i=1}^{\frac{T}{4}} \hat{\beta}_{2 i}-2^{\frac{1}{2}} \sum_{i=1}^{\frac{T}{2}} \hat{\beta}_{2 i}\right), \\
\hat{d}_{1,1}^{*(2,1)}=\frac{1}{T}\left(2^{\frac{3}{2}} \sum_{i=\frac{T}{2}+1}^{\frac{3 T}{4}} \hat{\beta}_{2 i}-2^{\frac{1}{2}} \sum_{i=\frac{T}{2}+1}^{T} \hat{\beta}_{2 i}\right) .
\end{gathered}
$$

Assim, das Equações 4.64, 4.61, 4.62, 4.65 e 4.66, temos que o estimador de máxima verossimilhança de $\mathbf{W}_{J}$ normalizado, utilizando $J=1$, é dado por:

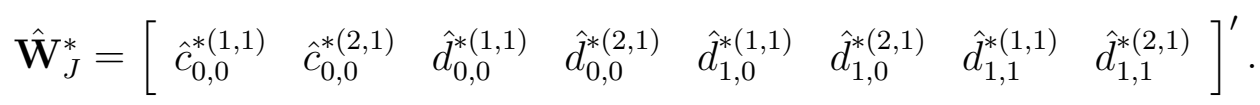

De maneira geral, para $n=2, r=1$ e $J$ qualquer, teremos:

$$
\hat{\mathbf{W}}_{J}^{*}=\left[\begin{array}{lllllll}
\hat{c}_{0,0}^{*(1,1)} & \hat{c}_{0,0}^{*(2,1)} & \hat{d}_{0,0}^{*(1,1)} & \hat{d}_{0,0}^{*(2,1)} & \ldots & \hat{d}_{J, 2^{J}-1}^{*(1,1)} & \hat{d}_{J, 2^{J}-1}^{*(2,1)}
\end{array}\right]^{\prime} .
$$

Os estimadores normalizados dos demais parâmetros do modelo são dados a seguir, independente do valor de $J$ escolhido:

$$
\begin{gathered}
\hat{\boldsymbol{\alpha}}^{*}=S_{01, T}^{(J)} \hat{\mathbf{W}}_{J}^{*}\left(\hat{\mathbf{W}}_{J}^{*^{\prime}} \mathbf{S}_{11, T}^{(J)} \hat{\mathbf{W}}_{J}^{*}\right)^{-1} \\
\hat{\boldsymbol{\Gamma}}^{*}=\left(\Delta Y-\hat{\boldsymbol{\alpha}}^{*} \hat{\mathbf{W}}_{J}^{*^{\prime}} Y_{-1}^{(J)}\right) \Delta X^{\prime}\left(\Delta X_{t} \Delta X^{\prime}\right)^{-1} \\
\hat{\boldsymbol{\Sigma}}_{u}^{*}=\frac{1}{T}\left(\Delta Y-\hat{\boldsymbol{\alpha}}^{*} \hat{\mathbf{W}}_{J}^{*^{\prime}} Y_{-1}^{(J)}-\hat{\boldsymbol{\Gamma}}^{*} \Delta X\right)\left(\Delta Y-\hat{\boldsymbol{\alpha}}^{*} \hat{\mathbf{W}}_{J}^{*^{\prime}} Y_{-1}^{(J)}-\hat{\boldsymbol{\Gamma}}^{*} \Delta X\right)^{\prime} .
\end{gathered}
$$

Vale ressaltar que, para obter os estimadores não normalizados $\boldsymbol{\alpha}, \boldsymbol{\Gamma}$ e $\boldsymbol{\Sigma}_{u}$, basta substituir $\mathbf{W}_{J}^{*^{\prime}}$ por $\mathbf{W}_{J}^{\prime}$ nas expressões $4.69,4.70$ e 4.71 .

\subsubsection{Caso especial - Modelo sem diferenças defasadas}

Temos interesse em trabalhar também com um modelo mais simples, onde não incluímos o termo de defasagens $\Gamma \Delta X_{t}$ na Equação 4.3. Para este caso, trabalhamos com o modelo

$$
\Delta Y_{t}=\boldsymbol{\alpha} \boldsymbol{\beta}_{t}^{\prime} Y_{t-1}^{(J)}+u_{t}
$$

Vamos apresentar a seguir os estimadores de máxima verossimilhança para este caso particular, seguindo de perto a metodologia apresentada em Lutkepohl (2005). Aqui, supomos $\boldsymbol{\alpha}$ e $\boldsymbol{\Sigma}_{u}$ desconhecidos. Construímos então a seguinte matriz:

$$
\left(\sum_{t=1}^{T} Y_{t-1}^{(J)} Y_{t-1}^{(J)^{\prime}}\right)^{-1 / 2}\left(\sum_{t=1}^{T} Y_{t-1}^{(J)} \Delta Y_{t}^{\prime}\right)\left(\sum_{t=1}^{T} \Delta Y_{t} \Delta Y_{t}^{\prime}\right)\left(\sum_{t=1}^{T} \Delta Y_{t} Y_{t-1}^{(J)^{\prime}}\right)\left(\sum_{t=1}^{T} Y_{t-1}^{(J)} Y_{t-1}^{(J)^{\prime}}\right)^{-1 / 2}
$$


cujos autovalores são $\hat{\lambda}_{1}^{(J)} \geq \hat{\lambda}_{2}^{(J)} \geq \ldots \geq \hat{\lambda}_{n}^{(J)}$ e respectivos autovetores ortonormais $\hat{\boldsymbol{v}}_{1}^{(J)}, \hat{\boldsymbol{v}}_{2}^{(J)}, \ldots, \hat{\boldsymbol{v}}_{n}^{(J)}$.

Com base na matriz apresentada na Equação 4.73, temos que o estimador de máxima verossimilhança de $\mathbf{W}_{J}$ para o modelo apresentado na Equação 4.72 é dado por

$$
\hat{\mathbf{W}}_{J}=\left[\begin{array}{llll}
\hat{\boldsymbol{v}}_{1}^{(J)} & \hat{\boldsymbol{v}}_{2}^{(J)} & \ldots & \hat{\boldsymbol{v}}_{r}^{(J)}
\end{array}\right]^{\prime}\left(Y_{-1}^{(J)} Y_{-1}^{(J)^{\prime}}\right)^{-1 / 2} .
$$

Logo, o estimador de máxima verossimilhança de $\beta_{t}$ é o mesmo apresentado na Equação 4.52 (ou 4.53), com os termos $\mathbf{c}_{\mathbf{0}, \mathbf{0}}, \mathbf{d}_{0,0}, \mathbf{d}_{1,0}, \ldots \mathbf{d}_{J, 2^{J}-1}$ obtidos na Equação 4.74.

De maneira análoga, utilizando a Equação 4.54, obtemos o estimador normalizado $\hat{\boldsymbol{\beta}}_{t}^{*}$. O método utilizado para encontrar o estimador normalizado $\hat{\mathbf{W}}_{J}^{*}$ é o mesmo apresentado anteriormente, dependendo da escolha de $J$.

O estimador de máxima verossimilhança normalizado de $\boldsymbol{\alpha}$ é dado por

$$
\hat{\boldsymbol{\alpha}}^{*}=\left(\sum_{t=1}^{T} \Delta Y_{t} Y_{t-1}^{(J)^{\prime}} \mathbf{W}_{J}^{*}\right)\left(\sum_{t=1}^{T} \mathbf{W}_{J}^{*^{\prime}} Y_{t-1}^{(J)} Y_{t-1}^{(J)^{\prime}} \mathbf{W}_{J}^{*}\right)^{-1}
$$

Os demais estimadores do modelo são obtidos via mínimos quadrados irrestritos:

$$
\hat{\boldsymbol{\Pi}}^{*}=\left(\sum_{t=1}^{T} \Delta Y_{t} Y_{t-1}^{(J)^{\prime}}\right)\left(\sum_{t=1}^{T} Y_{t-1}^{(J)} Y_{t-1}^{(J)^{\prime}}\right)^{-1}
$$

$\mathrm{e}$

$$
\hat{\mathbf{\Sigma}}_{u}^{*}=\sum_{t=1}^{T}\left(\Delta Y_{t}-\Pi^{*} Y_{t-1}^{(J)}\right)\left(\Delta Y_{t}-\Pi^{*} Y_{t-1}^{(J)}\right)^{\prime}
$$

Vale ressaltar mais uma vez que, os para obter os estimadores não normalizados $\hat{\boldsymbol{\alpha}}, \hat{\boldsymbol{\Gamma}}$ e $\hat{\boldsymbol{\Sigma}}_{u}$, basta substituir $\hat{\mathbf{W}}_{J}^{*^{\prime}}$ por $\hat{\mathbf{W}}_{J}$ nas expressões $4.75,4.76$ e 4.77 .

\subsection{Teste de razão de verossimilhanças}

Ao analisar um conjunto de dados cointegrados, sejam simulados ou reais, gostaríamos de verificar se as relações de cointegração variam com o tempo. Semelhante à metodologia empregada em Bierens e Martins (2010), utilizamos um teste de razão de verossimilhanças com este intuito. Desta forma, estamos interessados em testar as seguintes hipóteses:

$$
\left\{\begin{array}{ll}
\boldsymbol{H}_{\mathbf{0}}: & \text { Cointegração invariante com o tempo (C.I.), ou seja, } \boldsymbol{\Pi}_{J}=\Pi=\boldsymbol{\alpha} \boldsymbol{\beta}^{\prime} \\
\boldsymbol{H}_{\boldsymbol{A}}: & \text { Cointegração variando com o tempo (C.V.), ou seja, } \boldsymbol{\Pi}_{J}=\boldsymbol{\alpha} \mathbf{W}_{J}^{\prime}
\end{array} .\right.
$$

Para a hipótese nula $\boldsymbol{H}_{\mathbf{0}}, \boldsymbol{\alpha}$ e $\boldsymbol{\beta}$ são matrizes fixadas $(n \times r)$ de posto $r$. Já para a hipótese $\boldsymbol{H}_{\boldsymbol{A}}, \boldsymbol{\alpha}$ é dada como em $\boldsymbol{H}_{\mathbf{0}}$ e $\mathbf{W}_{J}^{\prime}$ é definida em 4.8 . 
Dada a representação do modelo $W A V E C$ da Equação 4.5, com vetor normalizado $\mathbf{W}_{J}^{*}$, temos que a hipótese nula $\boldsymbol{H}_{\mathbf{0}}$ corresponde a

$$
\mathbf{W}_{J}^{*^{\prime}}=\left(\boldsymbol{\beta}^{\prime}, \mathbf{0}\right)
$$

com a matriz de cointegração invariante com o tempo $\boldsymbol{\beta}(n \times r)$ e a matriz nula $\mathbf{0}\left(r \times n 2^{J}\right)$, tal que $\mathbf{W}_{J}^{*^{\prime}} Y_{-1}^{(J)}=\boldsymbol{\beta}^{\prime} Y_{-1}$.

O maior valor que pode ser alcançado pela log-verossimilhança do modelo, para $\boldsymbol{\theta}=$ $\left(\boldsymbol{\alpha}, \mathbf{W}_{J}, \boldsymbol{\Sigma}_{u}\right)$, com a restrição $\boldsymbol{\Pi}_{J}=\boldsymbol{\alpha} \mathbf{W}_{J}^{\prime}$ é dado por:

$$
\mathscr{L}(\boldsymbol{\theta}, r, J)=-\frac{T n}{2} \ln (2 \pi)-\frac{T n}{2}-\frac{T n}{2} \ln \left(\left|\Sigma_{u}\right|\right)-\frac{T}{2} \sum_{i=1}^{r} \ln \left(1-\hat{\lambda}_{i}^{(J)}\right)
$$

Além disso, o maior valor que pode ser alcançado pela log-verossimilhança do modelo $V E C(p)$, para $\boldsymbol{\theta}=\left(\boldsymbol{\alpha}, \boldsymbol{\beta}, \boldsymbol{\Sigma}_{u}\right)$, com a restrição $\boldsymbol{\Pi}_{J}=\boldsymbol{\Pi}=\boldsymbol{\alpha} \boldsymbol{\beta}$ é dado por:

$$
\mathscr{L}(\boldsymbol{\theta}, r, 0)=-\frac{T n}{2} \ln (2 \pi)-\frac{T n}{2}-\frac{T n}{2} \ln \left(\left|\Sigma_{u}\right|\right)-\frac{T}{2} \sum_{i=1}^{r} \ln \left(1-\hat{\lambda}_{i}\right) .
$$

Assim, dados $r$ e $J$, a estatística do teste de razão de verossimilhanças de $H_{0}$ contra $H_{A}$, toma a forma

$$
\begin{aligned}
R V_{C V T}^{(J)} & =-2[\mathscr{L}(\boldsymbol{\theta}, r, 0)-\mathscr{L}(\boldsymbol{\theta}, r, J)] \\
& =-2\left[-\frac{T}{2} \sum_{i=1}^{r} \ln \left(1-\hat{\lambda}_{i}\right)+\frac{T}{2} \sum_{i=1}^{r} \ln \left(1-\hat{\lambda}_{i}^{(J)}\right)\right] \\
& =T \sum_{i=1}^{r}\left\{\ln \left(\frac{1-\hat{\lambda}_{i}}{1-\hat{\lambda}_{i}^{(J)}}\right)\right\}
\end{aligned}
$$

onde $R V_{C V T}^{(J)}$ é a estatística do teste de razão de verossimilhanças para relações de cointegração variando com o tempo.

\subsection{Testes bootstrap - Propriedades empíricas dos es- timadores}

Conforme apresentado em Martins (2016), foi utilizado o método wild do bootstrap para obter intervalos de confiança empíricos dos parâmetros do modelo. O método também foi utilizado em testes de razão de verossimilhanças para testar a hipótese de cointegração constante ao longo do tempo versus cointegração variando com o tempo. Este método 
bootstrap é semelhante ao método sieve, porém Martins (2005) e Martins (2016) demonstraram que o primeiro método é mais eficiente, principalmente para trabalhar com amostras pequenas.

O teste bootstrap wild para a cointegração variando com o tempo (CVT) é obtido ao seguir os seguintes passos (Martins, 2016):

1. Primeiro, geramos $B$ pseudo-perturbações $u_{t}^{b}$ para $t=1, \ldots, T$ e $b=1, \ldots, B$ dos resíduos estimados $\hat{u}_{t}$, de forma que

$$
u_{t}^{b}=\hat{u}_{t} \cdot \mathbf{w}_{t}
$$

onde $\left\{\mathbf{w}_{t}\right\}_{t=1}^{T}$ é uma sequência de escalares independentes seguindo uma distribuição $N(0,1)$.

2. Em seguida, construímos a amostra bootstrap $\left\{\Delta Y_{t}^{b}\right\}_{t=1}^{T}$ recursivamente da equação

$$
\Delta Y_{t}^{b}=\hat{\boldsymbol{\alpha}} \hat{\boldsymbol{\beta}}^{\prime} Y_{t-1}^{b}+\hat{\boldsymbol{\Gamma}} \Delta X_{t}^{b}+u_{t}^{b}
$$

com valores iniciais $Y_{t}^{b}=Y_{t}$, para $t=(1-p), \ldots, 0$.

3. Usando a amostra bootstrap $\left\{\Delta Y_{t}^{b}\right\}_{t=1}^{T}$, construímos as estatísticas de razão de verossimilhanças

$$
R V_{C V T}^{(J, b)}=T \sum_{i=1}^{r} \ln \left(\frac{1-\hat{\lambda}_{i}^{b}}{1-\hat{\lambda}_{i}^{(J, b)}}\right)
$$

onde os termos $\hat{\lambda}_{i}^{b}$ 's denotam as versões bootstrap dos autovalores generalizados ordenados $\hat{\lambda}_{i}$ 's.

4. Em seguida, a versão bootstrap para o intervalo de confiança de $95 \%$ percentil é usado como valor crítico de teste de 5\%. De maneira análoga, é possível calcular a versão bootstrap do valor-p para a estatística $R V_{C V T}^{(J)}$ usando a proporção

$$
p_{b}^{(J)}=\frac{1}{B} \sum_{b=1}^{B} \mathbb{I}\left(R V_{C V T}^{(J, b)}>R V_{C V T}^{(J)}\right)
$$

5. Por fim, para um nível de significância $\delta$, rejeitamos a hipótese nula de cointegração invariante com o tempo se $p_{b}^{(J)}<\delta$.

Desta forma, podemos obter intervalos de confiança e a estimação da distribuição dos estimadores dos parâmetros, que podem ser construídos com base na amostra dos estimadores bootstrap. 


\subsection{Paridade de Poder de Compra variando com o tempo}

Tratamos da PPC variando com o tempo em dois casos: considerando e não considerando o intercepto no modelo. No primeiro caso, o vetor de cointegração é assumido como variando com o tempo. Esta abordagem também é empregada por Bierens e Martins (2010). No segundo caso, apenas o intercepto varia com o tempo, sendo o vetor de cointegração assumido como constante. Em ambos os casos, $p_{t}$ representa o logarítmo do índice de preços do país local, $p_{t}^{*}$ representa o logarítmo do índice de preços para o país estrangeiro e $s_{t}$ representa o logarítmo da taxa de câmbio em moeda do país local por unidade da moeda estrangeira, tal qual definido na Seção 2.5.

\subsubsection{Modelo desconsiderando o intercepto}

Podemos reecrever a Equação 2.59 considerando o vetor de cointegração $\beta$ variando como tempo, ou seja, o parâmetro $\boldsymbol{\beta}$ é representado temporalmente por $\boldsymbol{\beta}_{t}=\left[\beta_{0 t} \beta_{1 t} \beta_{2 t}\right]$, para $t=1, \ldots, T$. Desta forma, temos

$$
\beta_{0 t} s_{t}=\beta_{1 t} p_{t}+\beta_{2 t} p_{t}^{*}+u_{t}
$$

Podemos reescrever a Equação 4.86 na forma de correção de erros, conforme apresentado em 4.5. Sem perda de generalidade, vamos considerar a matriz de observações $\Delta Y_{t}$ tomando $n=3, r=1$ e $t=1, \ldots, T$ :

$$
Y_{t}=\left[\begin{array}{c}
Y_{1 t} \\
Y_{2 t} \\
Y_{3 t}
\end{array}\right]=\left[\begin{array}{c}
s_{t} \\
p_{t} \\
p_{t}^{*}
\end{array}\right] \text { e } \Delta Y_{t}=\left[\begin{array}{c}
\Delta Y_{1 t} \\
\Delta Y_{2 t} \\
\Delta Y_{3 t}
\end{array}\right]=\left[\begin{array}{c}
\Delta s_{t} \\
\Delta p_{t} \\
\Delta p_{t}^{*}
\end{array}\right]
$$

Assim, 4.86 escrita na forma de correção de erros, com vetor de cointegração variando com o tempo é dada por:

$$
\Delta Y_{t}=\boldsymbol{\alpha} \mathbf{W}_{J}^{\prime} Y_{t-1}^{(J)}+\sum_{i=1}^{p-1} \boldsymbol{\Gamma}_{i} \Delta Y_{t-i}+u_{t}
$$

Note que para o modelo apresentado em 4.88, estamos desconsiderando o intercepto no modelo. Logo, para estimar os parâmetros de um modelo cointegrado, basta utilizar os procedimentos apresentados na Seção 4.3.

\subsubsection{Modelo considerando o intercepto variando com o tempo e matriz de cointegração fixa}

Para este caso, vamos considerar a Equação 2.61, $\operatorname{com} \eta=\eta_{t}$. Desta forma, temos que 


$$
S_{t}=e^{\eta_{t}} \cdot\left(P_{t}\right) \cdot\left(P_{t}^{*}\right)^{-1} \cdot e^{u_{t}}
$$

de onde podemos extrair

$$
\underbrace{\left(\frac{S_{t}}{e^{u_{t}}} \cdot \frac{P_{t}^{*}}{P_{t}}\right)}_{E_{t}}=e^{\eta_{t}} .
$$

Note que o modelo apresentado na Equação 4.89 generaliza o caso em $\eta$ não varia com o tempo (Equação 2.61). A Figura 4.1 mostra um esquema para a quantidade $e^{\eta}$ teórica (igual a 1 como referência) e estimada $e^{\hat{\eta}_{t}}$. Por simplicidade, omitimos o termo $e^{u_{t}}$ da comparação com o valor teórico.

Para este modelo, podemos interpretar os resultados considerando os seguintes casos para $e^{\eta_{t}}$ :

- Se $e^{\hat{\eta}_{t}}$ oscila em torno de 1 , então $\eta_{t} \approx 0$ para todo $t=1, \ldots, T$. Logo, deveremos trabalhar com outro modelo de cointegração que não contemple o intercepto;

- Se $e^{\hat{\eta}_{t}}$ oscilar de maneira não estacionária entre 0 e 1 ou oscilar acima de 1 , temos indícios de que devemos considerar o modelo com intercepto variando com tempo.

Na Figura 4.1 apresentamos um exemplo hipotético onde tal situação pode ocorrer.

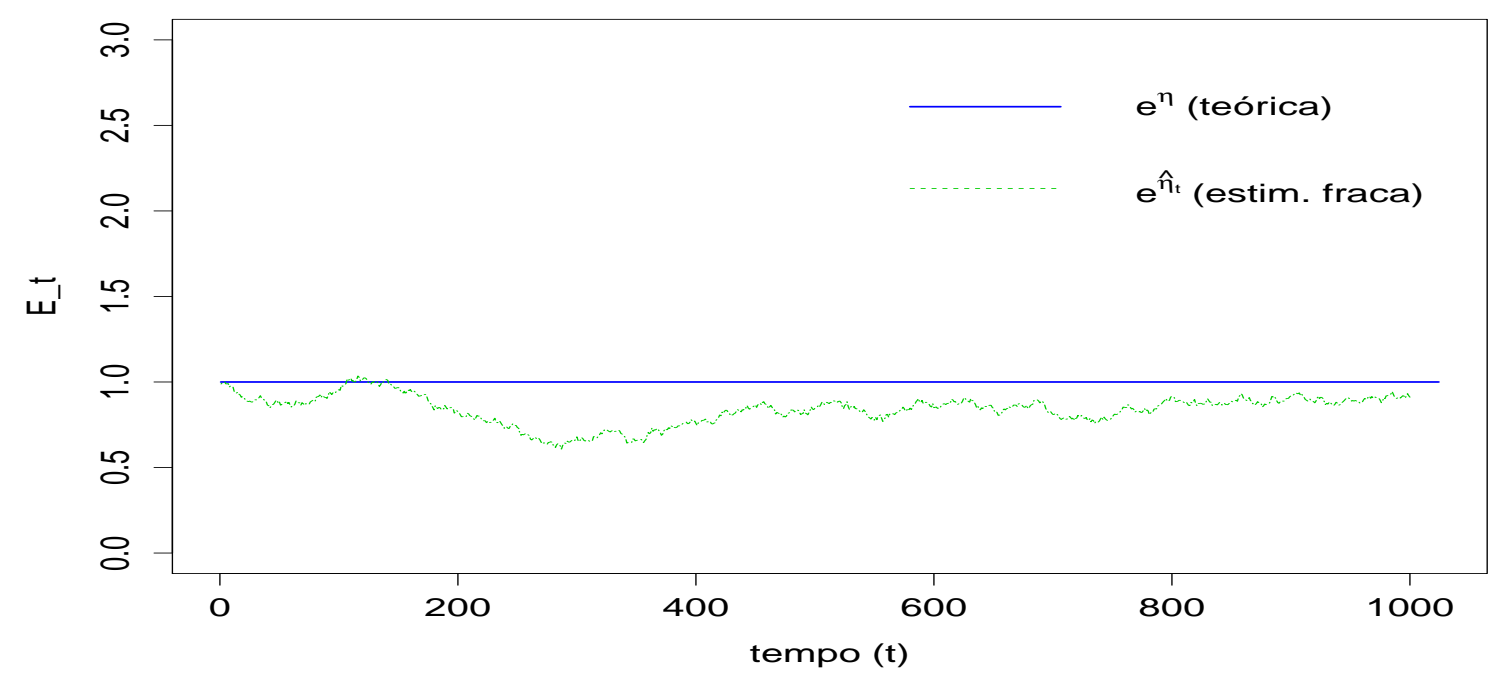

Figura 4.1: Esquema PPC teórica invariante como tempo versus estimada variando com o tempo.

Vamos construir este modelo de maneira similar à apresentada na Equação 4.3. Neste caso no entanto, vamos tomar $\boldsymbol{\alpha}$ e $\boldsymbol{\beta}$ como sendo matrizes fixas de ordem $(n \times r)$. Note que na Equação 4.3 era permitido ao vetor $\boldsymbol{\beta}$ variar com o tempo.

Para isso, vamos considerar a matriz de observações $\Delta Y_{t}$, para $n=3, r=1$ e $t=$ $1, \ldots, T$ : 


$$
Y_{t}=\left[\begin{array}{c}
Y_{1 t} \\
Y_{2 t} \\
Y_{3 t}
\end{array}\right]=\left[\begin{array}{c}
s_{t} \\
p_{t} \\
p_{t}^{*}
\end{array}\right] \text { e } \Delta Y_{t}=\left[\begin{array}{c}
\Delta Y_{1 t} \\
\Delta Y_{2 t} \\
\Delta Y_{3 t}
\end{array}\right]=\left[\begin{array}{c}
\Delta s_{t} \\
\Delta p_{t} \\
\Delta p_{t}^{*}
\end{array}\right]
$$

Podemos então reescrever o modelo dado na Equação 4.89 na forma de correção de erros, conforme descrito na Equação 4.92

$$
\Delta Y_{t}=\boldsymbol{\alpha}\left[\begin{array}{ll}
\boldsymbol{\beta}^{\prime} & \eta_{t}^{\prime}
\end{array}\right]\left[\begin{array}{c}
Y_{t-1} \\
1
\end{array}\right]+\boldsymbol{\Gamma} \Delta X_{t-1}+u_{t}
$$

Equivalentemente ao apresentado no Capítulo 4, vamos considerar desta vez o parâmetro $\eta_{t}$ variando com o tempo. Na Equação 4.93, reescrevemos $\eta_{t}$ como uma combinação de ondaletas pai e mãe:

$$
\eta_{t}=c_{0,0} \phi(t)+\sum_{j=0}^{J} \sum_{k=0}^{2^{j}-1} d_{j, k} \psi_{j, k}(t) .
$$

Substuindo 4.93 em 4.92, temos que

$$
\begin{aligned}
& \Delta Y_{t}=\boldsymbol{\alpha}\left[\begin{array}{lllll}
\boldsymbol{\beta}^{\prime} & c_{0,0}^{\prime} & d_{0,0}^{\prime} & \ldots & d_{J, 2^{J}-1}^{\prime}
\end{array}\right]\left[\begin{array}{c}
Y_{t-1} \\
\phi_{0,0}(t) \\
\psi_{0,0}(t) \\
\vdots \\
\psi_{J, 2^{J}-1}(t)
\end{array}\right]+\boldsymbol{\Gamma} \Delta X_{t-1}+u_{t} \\
& =\boldsymbol{\alpha} \underbrace{\left[\begin{array}{lllllll}
\beta_{1} & \beta_{2} & \beta_{3} & c_{0,0}^{\prime} & d_{0,0}^{\prime} & \cdots & d_{J, 2^{J}-1}^{\prime}
\end{array}\right]}_{W^{+^{\prime}}} \underbrace{\left[\begin{array}{c}
s_{t} \\
p_{t} \\
p_{t}^{*} \\
\phi_{0,0}(t) \\
\psi_{0,0}(t) \\
\vdots \\
\psi_{J, 2^{J}-1}(t)
\end{array}\right]}_{Y_{t-1}^{+}}+\boldsymbol{\Gamma} \Delta X_{t-1}+u_{t} \\
& =\boldsymbol{\alpha} \mathbf{W}^{+^{\prime}} Y_{t-1}^{(J)+}+\boldsymbol{\Gamma} \Delta X_{t-1}+u_{t} .
\end{aligned}
$$

Estamos fazendo a suposição de simetria de preços e de normalidade para o modelo, o que é equivalente a trabalhar $\operatorname{com} \beta$ dado em 2.58. Ao fixar o vetor $\beta$ desta forma, estamos trabalhando com um modelo com restrição de parâmetros. Neste caso, vamos nos valer da teoria apresentada em Lutkepohl (2005) para estimar os demais parâmetros do modelo considerando esta restrição. 
Devemos verificar se as restrições para a matriz de cointegração podem ser escritas da seguinte forma:

$$
\mathbf{W}^{+}=\mathbf{H} \cdot \boldsymbol{\varphi}
$$

onde $\mathbf{H}$ é uma matriz fixa e conhecida de ordem $n \times \ell$ e $\varphi$ é uma matriz de ordem $\ell \times r$, com $\ell \geq r$. Neste caso, as estimativas de máxima verossimilhança dos parâmetros do modelo podem ser obtidas, bastando apenas considerar (Lutkepohl, 2005)

$$
Y_{-1}^{(J)+}=\mathbf{H}^{\prime} \cdot Y_{-1}^{(J)+}
$$

na construção das matrizes $S_{i j} i, j=0,1$, já construídas na Seção 4.4.

Como estamos trabalhando com o modelo de PPC, fixamos, sem perda de generalidade, $n=3$ e $r=1$. Trabalhamos também com as restrições: $\beta_{1}=1$ (modelo normalizado), $\beta_{2}=-\beta_{1}, \beta_{3}=\beta_{1}$ e $\eta_{t} \neq 0$.

Desta forma, podemos escrever:

$$
\begin{aligned}
& W^{+}=\left[\begin{array}{c}
\boldsymbol{\beta} \\
\mathbf{W}_{J}
\end{array}\right]=\left[\begin{array}{c}
\beta_{1} \\
\beta_{2} \\
\beta_{3} \\
c_{0,0} \\
d_{0,0} \\
\vdots \\
d_{J, 2^{J}-1}
\end{array}\right]=\left[\begin{array}{c}
\beta_{1} \\
-\beta_{1} \\
\beta_{1} \\
\hline c_{0,0} \\
d_{0,0} \\
\vdots \\
d_{J, 2^{J}-1}
\end{array}\right] \\
& =\left[\begin{array}{r|ccccc}
1 & 0 & 0 & \ldots & 0 & 0 \\
-1 & 0 & 0 & \ldots & 0 & 0 \\
1 & 0 & 0 & \ldots & 0 & 0 \\
\hline 0 & 1 & 0 & \ldots & 0 & 0 \\
0 & 0 & 1 & \ldots & 0 & 0 \\
\vdots & \vdots & \vdots & \ddots & 0 & 0 \\
0 & 0 & 0 & \ldots & 1 & 0 \\
0 & 0 & 1 & \ldots & 0 & 1
\end{array}\right] \cdot\left[\begin{array}{c}
\beta_{0} \\
c_{0,0} \\
d_{0,0} \\
\vdots \\
d_{J, 2^{J}-1}
\end{array}\right]= \\
& =\underbrace{\left[\begin{array}{c|c}
\mathbf{R} & \mathbf{O}_{1} \\
\hline \mathbf{O}_{2} & \mathbf{I}_{w}
\end{array}\right]}_{\boldsymbol{H}} \underbrace{\left[\begin{array}{c}
\beta_{1} \\
\mathbf{W}_{J}
\end{array}\right]}_{\varphi}
\end{aligned}
$$

onde $\mathbf{R}$ é a matriz de restrições de ordem $n \times r, \mathbf{O}_{1}$ é uma matriz de zeros com ordem $n \times 2^{J+1}, \mathbf{O}_{2}$ é uma matriz de zeros com ordem $2^{J+1} \times r, \mathbf{I}_{w}$ é uma matriz identidade com ordem $2^{J+1} \times 2^{J+1}$ e $\mathbf{W}_{J}$ é a matriz com coeficientes de ondaletas.

Logo, para estimar os parâmetros do modelo, podemos construir as matrizes: 


$$
\begin{gathered}
\mathbf{S}_{00, T}=\frac{1}{T} \sum_{t=1}^{T} \Delta Y_{t}\left(\Delta Y_{t}\right)^{\prime}-\widehat{\mathbf{\Sigma}}_{X \Delta Y}^{\prime}\left(\widehat{\boldsymbol{\Sigma}}_{X X}\right)^{-1} \widehat{\mathbf{\Sigma}}_{X \Delta Y} \\
\mathbf{S}_{11, T}^{(J)+}=\frac{1}{T} \sum_{t=1}^{T} Y_{t-1}^{(J)+}\left(Y_{t-1}^{(J)+}\right)^{\prime}-\widehat{\mathbf{\Sigma}}_{X Y(J)}^{\prime}\left(\widehat{\boldsymbol{\Sigma}}_{X X}\right)^{-1} \widehat{\mathbf{\Sigma}}_{X Y^{(J)+}}, \\
\mathbf{S}_{01, T}^{(J)+}=\frac{1}{T} \sum_{t=1}^{T} \Delta Y_{t}\left(Y_{t-1}^{(J)+}\right)^{\prime}-\widehat{\mathbf{\Sigma}}_{X \Delta Y}^{\prime}\left(\widehat{\mathbf{\Sigma}}_{X X}\right)^{-1} \widehat{\mathbf{\Sigma}}_{X Y^{(J)}}, \\
\mathbf{S}_{10, T}^{(J)+}=\left(\mathbf{S}_{01, T}^{(J)+}\right)^{\prime}
\end{gathered}
$$

com:

$$
\begin{gathered}
\widehat{\Sigma}_{X X}=\frac{1}{T} \sum_{t=1}^{T} \Delta X_{t}\left(\Delta X_{t}\right)^{\prime}, \\
\widehat{\Sigma}_{X \Delta Y}=\frac{1}{T} \sum_{t=1}^{T} \Delta X_{t}\left(\Delta Y_{t}\right)^{\prime}, \\
\widehat{\Sigma}_{X Y_{t-1}^{+}}=\frac{1}{T} \sum_{t=1}^{T} \Delta X_{t}\left(Y_{t-1}^{(J)+}\right)^{\prime} .
\end{gathered}
$$

Desta forma, utilizando as técnicas apresentadas nos Capítulos 2 e 4, podemos ver que os estimadores dos parâmetros do modelo 4.94 são dados a seguir:

$$
\tilde{W}^{+}=\mathbf{H} \tilde{\varphi}=\left[\begin{array}{lllllll}
\tilde{\beta}_{1} & -\tilde{\beta}_{1} & \tilde{\beta}_{1} & \tilde{c}_{0,0} & \tilde{d}_{0,0} & \ldots & \tilde{d}_{J, 2^{J}-1}
\end{array}\right]^{\prime}
$$

onde $H$ é uma matriz de ordem $n+2^{J+1} \times 2^{J+1}+r$ e $\hat{\varphi}$ é uma matriz de ordem $2^{J+1}+r \times r$.

$$
\begin{gathered}
\tilde{\eta}_{t}=\tilde{c}_{0,0} \phi(t)+\sum_{j=0}^{J} \sum_{k=0}^{2^{j}-1} \tilde{d}_{j, k} \psi_{j, k}(t), \\
\tilde{\boldsymbol{\beta}}=\left[\begin{array}{llll}
\tilde{\beta}_{1} & -\tilde{\beta}_{1} & \tilde{\beta}_{1} & \tilde{\eta}_{t}
\end{array}\right]^{\prime} .
\end{gathered}
$$

Os estimadores normalizados dos demais parâmetros do modelo são dados a seguir, independente do valor de $J$ escolhido:

$$
\begin{gathered}
\tilde{\boldsymbol{\alpha}}^{*}=S_{01, T}^{(J)} \tilde{\varphi}\left(\tilde{\varphi}^{\prime} \mathbf{S}_{11, T}^{(J)} \tilde{\varphi}\right)^{-1} \\
\tilde{\boldsymbol{\Gamma}}^{*}=\left(\Delta Y-\hat{\boldsymbol{\alpha}}^{*} \tilde{\varphi}^{\prime} H Y_{t-1}^{(J)+}\right) \Delta X^{\prime}\left(\Delta X_{t} \Delta X^{\prime}\right)^{-1} \\
\tilde{\boldsymbol{\Sigma}}_{u}^{*}=\frac{1}{T}\left(\Delta Y-\tilde{\boldsymbol{\alpha}}^{*} \tilde{\varphi}^{\prime} H Y_{t-1}^{(J)+}-\tilde{\boldsymbol{\Gamma}}^{*} \Delta X\right)\left(\Delta Y-\tilde{\boldsymbol{\alpha}}^{*} \tilde{\varphi}^{\prime} H Y_{t-1}^{(J)+}-\tilde{\boldsymbol{\Gamma}}^{*} \Delta X\right)^{\prime} .
\end{gathered}
$$




\section{Capítulo 5}

\section{Relações de cointegração simuladas}

Este capítulo dedica-se ao estudo dos quantis empíricos e do poder empírico do teste de razão de verossimilhanças para testar a hipótese de cointegração variando com o tempo. Além disso, para ilustrar a aplicação do modelo, são apresentados seis modelos simulados, divididos em dois casos: os cinco primeiros modelos sem defasagem, enquanto o sexto exemplo inclui uma defasagem.

\subsection{Quantis empíricos e poder empírico do teste de razão de verossimilhanças}

Em Bierens e Martins (2010), os autores modelaram o vetor de cointegração temporalmente utilizando polinômios de Chebyshev. Eles também demonstraram que, assintoticamente, a estatística de razão de verossimilhanças segue uma distribuição Qui-quadrado com $v=m n r$ graus de liberdade, denotado por $\chi_{\nu=m n r}^{2}$, sendo $m$ o grau do polinômio utilizado, com $n$ e $r$ sendo os parâmetros do modelo cointegrado usual.

Para o modelo apresentado na Seção 4.1, com base em resultados numéricos, vemos a seguir que a estatística de razão de verossimilhanças $R V_{C V T}^{(J)}$ segue aproximadamente uma distribuição $\chi^{2}$, com o número de graus de liberdade adaptado ao caso com ondaletas. $\mathrm{O}$ número de graus de liberdade, tal como descrito para o caso com polinômios de Chebyshev, deve levar em conta o número de parâmetros $n$ e $r$ do modelo de cointegração usual, além do nível $J$ utilizado na decomposição em ondaletas. Temos um forte indicativo numérico de que $R V_{C V T}^{(J)}$ seguirá uma distribuição $\chi_{\nu}^{2}$, com $\nu=(N-1) n r$, sendo $N$ o número de coeficientes de ondaletas utilizado na decomposição da matriz $\boldsymbol{\beta}_{t}$, considerando um único nível para a ondaleta pai e diversos níveis para a ondaleta mãe.

Como usual para testes de razão de verossimilhanças para hipóteses encaixadas, o número de graus de liberdade corresponde ao número de parâmetros a mais no modelo completo, com relação ao modelo sob a hipótese nula de que o vetor de cointegração é invariante com o tempo (Sen et al., 2010). Neste caso, temos que $N=2^{J+1}$ e então rees- 
crevemos a expressão para $\nu$ como $\nu=\left(2^{J+1}-1\right) n r$.

Para confirmar estes resultados, apresentamos a seguir os quantis empíricos da estatística $R V_{C V T}^{(J)}$, expressão 4.81, obtidos via simulações de Monte Carlo, sob a hipótese nula de cointegração invariante com o tempo, para diferentes tamanhos amostrais. Além disso, aumentamos de maneira consecutiva a quantidade de níveis, de forma a verificar a aproximação numérica para cada caso.

Nas Tabelas 5.1, 5.2 e 5.3 apresentamos medidas resumo para a estatística de razão de verossimilhanças, considerando as bases de ondaletas "Haar", "Daubechies D2" e "Symmlet S8" respectivamente. Em cada caso, consideramos $N=1000$ séries simuladas, $n=2$, $r=1$ e resoluções $J=0,1,2,3,4$ e 5. Além disso, apresentamos os quantis teóricos, assumindo a validade da aproximação para a distribuição qui-quadrado, apenas para referência. É possível notar que os valores empíricos se aproximam dos valores teóricos à medida que aumentamos o tamanho da amostra nas simulações.

Tabela 5.1: Quantis, média e erros padrão empíricos e teóricos para a estatística $R V_{C V T}^{(J)}$, utilizando a ondaleta Haar, obtidos sob $H_{0}$ (cointegração invariante com o tempo).

\begin{tabular}{|c|c|c|c|c|c|c|c|c|}
\hline & \multicolumn{6}{|c|}{ Quantis } & \multicolumn{2}{|c|}{ Estatísticas } \\
\hline Haar & Série & $50 \%$ & $90 \%$ & $95 \%$ & $97,50 \%$ & $99 \%$ & Média & E.P. \\
\hline \multirow{4}{*}{$\mathrm{J}=0$} & $\mathrm{~T}=256$ & 1,5331 & 5,0808 & 6,3319 & 7,8117 & 9,1519 & 2,1794 & 2,0951 \\
\hline & $\mathrm{T}=512$ & 1,4650 & 4,5812 & 6,4668 & 7,5291 & 9,5210 & 2,0629 & 2,0922 \\
\hline & $\mathrm{T}=1024$ & 1,3715 & 4,5235 & 6,0488 & 7,8135 & 9,5107 & 2,0253 & 2,0478 \\
\hline & $\mathrm{T}=4096$ & 1,4307 & 4,6828 & 6,0457 & 7,4279 & 9,3486 & 2,0282 & 2,0070 \\
\hline \multicolumn{2}{|c|}{ Referência $\left(\chi_{\nu=2}^{2}\right)$} & 1,3863 & 4,6052 & 5,9915 & 7,3778 & 9,2103 & 2,0000 & 2,0000 \\
\hline \multirow{4}{*}{$\mathrm{J}=1$} & $\bar{T}=256$ & 5,3408 & 10,9814 & 13,0897 & 1014,6318 & 17,1862 & 6,1400 & 3,6387 \\
\hline & $\mathrm{T}=512$ & 5,3358 & 11,0977 & 12,9679 & 14,6323 & 17,0081 & 6,0687 & 3,5901 \\
\hline & $\mathrm{T}=1024$ & 5,4828 & 10,3698 & 12,2373 & 13,6369 & 17,4738 & 6,0034 & 3,5383 \\
\hline & $\mathrm{T}=4096$ & 5,2561 & 10,5053 & 12,4680 & 14,0449 & 16,7514 & 5,9860 & 3,4043 \\
\hline \multicolumn{2}{|c|}{ Referência $\left(\chi_{\nu=6}^{2}\right)$} & 5,3481 & 10,6446 & 12,5916 & 14,4494 & 16,8119 & 6,0000 & 3,4641 \\
\hline \multirow{4}{*}{$\mathrm{J}=2$} & $\mathrm{~T}=256$ & 214,0700 & 21,9893 & 24,7166 & 27,3619 & 29,5692 & 214,5921 & 5,5552 \\
\hline & $\mathrm{T}=512$ & 13,4342 & 21,5551 & 23,9261 & 27,2112 & 29,0954 & 14,1456 & 5,3745 \\
\hline & $\mathrm{T}=1024$ & 13,3982 & 21,1016 & 23,3077 & 25,9582 & 28,7944 & 14,0421 & 5,1675 \\
\hline & $\mathrm{T}=4096$ & 13,5789 & 21,3636 & 23,5763 & 25,6958 & 27,6109 & 14,1922 & 5,2199 \\
\hline \multicolumn{2}{|c|}{ Referência $\left(\chi_{\nu=14}^{2}\right)$} & 13,3393 & 21,0641 & 23,6848 & 26,1189 & 29,1412 & 14,0000 & 5,2915 \\
\hline \multirow{4}{*}{$\mathrm{J}=3$} & $\mathrm{~T}=256$ & 31,1564 & 43,1603 & 46,1009 & 50,4281 & 53,7648 & 31,7212 & 8,5084 \\
\hline & $\mathrm{T}=512$ & 29,9182 & 41,1800 & 44,8282 & 47,6313 & 50,9320 & 30,8221 & 7,5527 \\
\hline & $\mathrm{T}=1024$ & 29,2182 & 40,6644 & 43,6629 & 46,0810 & 50,9657 & 30,0528 & 7,5623 \\
\hline & $\mathrm{T}=4096$ & 29,6263 & 39,8035 & 43,5370 & 47,4515 & 51,4795 & 30,2828 & 7,8070 \\
\hline \multicolumn{2}{|c|}{ Referência $\left(\chi_{\nu=30}^{2}\right)$} & 29,3360 & 40,2560 & 43,7730 & 46,9792 & 50,8922 & 30,0000 & 7,7460 \\
\hline \multirow{4}{*}{$\mathrm{J}=4$} & $\mathrm{~T}=256$ & 68,1779 & 84,1041 & 89,5981 & 94,5710 & 101,5639 & 68,5021 & 12,3995 \\
\hline & $\mathrm{T}=512$ & 64,1821 & 79,2744 & 85,4435 & 89,2097 & 94,7515 & 64,6483 & 11,6602 \\
\hline & $\mathrm{T}=1024$ & 62,3479 & 77,0465 & 81,1736 & 85,9954 & 91,5495 & 62,9930 & 10,9880 \\
\hline & $\mathrm{T}=4096$ & 60,8178 & 77,8327 & 83,8082 & 88,7453 & 95,6391 & 62,5569 & 12,0995 \\
\hline \multicolumn{2}{|c|}{ Referência $\left(\chi_{\nu=62}^{2}\right)$} & 61,3346 & 76,6302 & 81,3810 & 85,6537 & 90,8015 & 62,0000 & 11,1355 \\
\hline \multirow{4}{*}{$\mathrm{J}=5$} & $\bar{T}=256$ & $\overline{162,2814}$ & $\overline{~ 190,6147}$ & 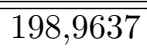 & $\overline{205,8693}$ & 217,4858 & $\overline{162,9720}$ & 21,3739 \\
\hline & $\mathrm{T}=512$ & 136,5297 & 160,8637 & 166,5737 & 173,6013 & 179,9645 & 137,7817 & 17,3981 \\
\hline & $\mathrm{T}=1024$ & 130,2180 & 151,9722 & 159,4935 & 165,5070 & 174,6093 & 130,9665 & 16,9904 \\
\hline & $\mathrm{T}=4096$ & 127,3415 & 148,7465 & 155,4227 & 160,9546 & 170,7986 & 127,8181 & 16,3328 \\
\hline \multicolumn{2}{|c|}{ Referência $\left(\chi_{\nu=126}^{2}\right)$} & 125,3340 & 146,7241 & 153,1979 & 158,9624 & 165,8410 & 126,0000 & 15,8745 \\
\hline
\end{tabular}


Tabela 5.2: Quantis, média e erros padrão empíricos e teóricos para a estatística $R V_{C V T}^{(J)}$, utilizando a ondaleta Daubechies DQ, obtidos sob $H_{0}$ (cointegração invariante com o tempo).

\begin{tabular}{|c|c|c|c|c|c|c|c|c|}
\hline & \multicolumn{6}{|c|}{ Quantis } & \multicolumn{2}{|c|}{ Estatísticas } \\
\hline D2 & Série & $50 \%$ & $90 \%$ & $95 \%$ & $97,50 \%$ & $99 \%$ & Média & E.P. \\
\hline \multirow{4}{*}{$\mathrm{J}=0$} & $\mathrm{~T}=256$ & 1,4469 & 4,7679 & 6,2985 & 7,9434 & 10,4686 & 2,0756 & 2,1220 \\
\hline & $\mathrm{T}=512$ & 1,4429 & 4,5655 & 5,5706 & 6,8917 & 8,2025 & 1,9681 & 1,9291 \\
\hline & $\mathrm{T}=1024$ & 1,4074 & 4,6730 & 6,0493 & 7,0626 & 9,2955 & 2,0645 & 2,0229 \\
\hline & $\mathrm{T}=4096$ & 1,3568 & 4,6494 & 5,9239 & 7,5688 & 10,0547 & 2,0036 & 2,0731 \\
\hline \multicolumn{2}{|c|}{ Referência $\left(\chi_{\nu=2}^{2}\right)$} & 1,3863 & 4,6052 & 5,9915 & 7,3778 & 9,2103 & 2,0000 & 2,0000 \\
\hline \multirow{4}{*}{$\mathrm{J}=1$} & $\bar{T}=256$ & $\overline{5,5584}$ & $\begin{array}{ll}11,0343 \\
\end{array}$ & $\overline{12,8834}$ & $\begin{array}{l}15,0627 \\
\end{array}$ & $\begin{array}{ll}16,8188 \\
\end{array}$ & 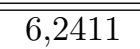 & 3,6037 \\
\hline & $\mathrm{T}=512$ & 5,5431 & 10,7039 & 12,9457 & 14,8148 & 15,9527 & 6,1758 & 3,4581 \\
\hline & $\mathrm{T}=1024$ & 5,3163 & 10,9899 & 12,6366 & 14,3497 & 16,8988 & 6,0668 & 3,5540 \\
\hline & $\mathrm{T}=4096$ & 5,5419 & 10,8975 & 12,8406 & 15,5534 & 17,9010 & 6,1712 & 3,5531 \\
\hline \multicolumn{2}{|c|}{ Referência $\left(\chi_{\nu=6}^{2}\right)$} & 5,3481 & 10,6446 & 12,5916 & 14,4494 & 16,8119 & 6,0000 & 3,4641 \\
\hline \multirow{4}{*}{$J=2$} & $\bar{T}=256$ & $\overline{14,2075}$ & 222,6577 & 24,7853 & $=27,3610$ & 30,6246 & "14,8311 & $\overline{5,6439}$ \\
\hline & $\mathrm{T}=512$ & 13,2069 & 20,4318 & 23,2855 & 26,6914 & 29,0014 & 13,9270 & 5,1862 \\
\hline & $\mathrm{T}=1024$ & 13,6804 & 21,0715 & 23,7497 & 26,3301 & 30,0294 & 14,1737 & 5,3119 \\
\hline & $\mathrm{T}=4096$ & 13,6204 & 21,3351 & 24,0338 & 25,8896 & 28,9691 & 14,2017 & 5,2083 \\
\hline \multicolumn{2}{|c|}{ Referência $\left(\chi_{\nu=14}^{2}\right)$} & 13,3393 & 21,0641 & 23,6848 & 26,1189 & 29,1412 & 14,0000 & 5,2915 \\
\hline \multirow{4}{*}{$\mathrm{J}=3$} & $\mathrm{~T}=256$ & 30,4361 & 41,4003 & $\overline{45,4042}$ & 48,7466 & 53,7520 & 31,0373 & 8,0686 \\
\hline & $\mathrm{T}=512$ & 29,7781 & 40,0918 & 43,6366 & 46,8815 & 50,3867 & 30,5845 & 7,6001 \\
\hline & $\mathrm{T}=1024$ & 29,2617 & 39,5707 & 43,4769 & 47,8512 & 51,8415 & 30,0128 & 7,5995 \\
\hline & $\mathrm{T}=4096$ & 29,2841 & 39,6018 & 43,0733 & 45,3271 & 48,5172 & 29,8547 & 7,5030 \\
\hline \multicolumn{2}{|c|}{ Referência $\left(\chi_{\nu=30}^{2}\right)$} & 29,3360 & 40,2560 & 43,7730 & 46,9792 & 50,8922 & 30,0000 & 7,7460 \\
\hline \multirow{4}{*}{$\mathrm{J}=4$} & $\mathrm{~T}=256$ & 67,4666 & 84,5747 & 90,7665 & 94,5624 & $\begin{array}{l}99,7292 \\
\end{array}$ & 68,3378 & 12,4530 \\
\hline & $\mathrm{T}=512$ & 63,0113 & 79,8812 & 85,1758 & 89,0907 & 93,5631 & 64,1146 & 11,7004 \\
\hline & $\mathrm{T}=1024$ & 62,3615 & 78,2182 & 82,3188 & 86,4973 & 89,5525 & 62,8876 & 11,4277 \\
\hline & $\mathrm{T}=4096$ & 61,6559 & 77,6814 & 82,0570 & 85,8391 & 93,2429 & 62,2104 & 11,5890 \\
\hline \multicolumn{2}{|c|}{ Referência $\left(\chi_{\nu=62}^{2}\right)$} & 61,3346 & 76,6302 & 81,3810 & 85,6537 & 90,8015 & 62,0000 & 11,1355 \\
\hline \multirow{4}{*}{$\mathrm{J}=5$} & $\mathrm{~T}=256$ & 164,4959 & 194,2850 & 200,5327 & 209,1355 & 216,5904 & 164,9336 & 21,5837 \\
\hline & $\mathrm{T}=512$ & 137,4613 & 160,2970 & 167,2624 & 172,3542 & 180,5752 & 138,1466 & 17,0075 \\
\hline & $\mathrm{T}=1024$ & 128,8077 & 151,1039 & 157,8569 & 164,4674 & 172,6155 & 129,7655 & 16,6212 \\
\hline & $\mathrm{T}=4096$ & 126,3531 & 146,4014 & 154,8341 & 159,3293 & 167,0838 & 127,0892 & 16,0386 \\
\hline \multicolumn{2}{|c|}{ Referência $\left(\chi_{\nu=126}^{2}\right)$} & 125,3340 & 146,7241 & 153,1979 & 158,9624 & 165,8410 & 126,0000 & 15,8745 \\
\hline
\end{tabular}


Tabela 5.3: Quantis, média e erros padrão empíricos e teóricos para a estatística $R V_{C V T}^{(J)}$, utilizando a ondaleta Symmlet $S 8$, obtidos sob $H_{0}$ (cointegração invariante com o tempo).

\begin{tabular}{|c|c|c|c|c|c|c|c|c|}
\hline & \multicolumn{6}{|c|}{ Quantis } & \multicolumn{2}{|c|}{ Estatísticas } \\
\hline 58 & Série & $50 \%$ & $90 \%$ & $95 \%$ & $97,50 \%$ & $99 \%$ & Média & E.P. \\
\hline \multirow{4}{*}{$\mathrm{J}=0$} & $\mathrm{~T}=256$ & 1,3699 & 4,6927 & 6,1980 & 7,6044 & 9,1463 & 1,9940 & 2,0510 \\
\hline & $\mathrm{T}=512$ & 1,4319 & 4,8458 & 6,7432 & 7,8683 & 9,0945 & 2,0567 & 2,0885 \\
\hline & $\mathrm{T}=1024$ & 1,4278 & 4,4900 & 6,1164 & 7,4844 & 9,7964 & 2,0152 & 2,0146 \\
\hline & $\mathrm{T}=4096$ & 1,3294 & 4,2946 & 5,4921 & 7,0027 & 8,3708 & 1,9051 & 1,8610 \\
\hline \multicolumn{2}{|c|}{ Referência $\left(\chi_{\nu=2}^{2}\right)$} & 1,3863 & 4,6052 & 5,9915 & 7,3778 & 9,2103 & 2,0000 & 2,0000 \\
\hline \multirow{4}{*}{$\mathrm{J}=1$} & $\bar{T}=256$ & $\overline{5,3942}$ & 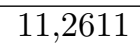 & 13,4225 & $\begin{array}{l}14,9851 \\
\end{array}$ & $\bar{~} 16,8302$ & $\overline{6,1070}$ & $3,5,5860$ \\
\hline & $\mathrm{T}=512$ & 5,6685 & 10,6881 & 12,4714 & 14,1678 & 15,8481 & 6,2063 & 3,3853 \\
\hline & $\mathrm{T}=1024$ & 5,3798 & 10,4150 & 12,2721 & 14,5549 & 16,4627 & 5,9665 & 3,3978 \\
\hline & $\mathrm{T}=4096$ & 5,4048 & 10,9299 & 12,6239 & 14,1889 & 17,3246 & 6,0481 & 3,5072 \\
\hline \multicolumn{2}{|c|}{ Referência $\left(\chi_{\nu=6}^{2}\right)$} & 5,3481 & 10,6446 & 12,5916 & 14,4494 & 16,8119 & 6,0000 & 3,4641 \\
\hline \multirow{4}{*}{$J=2$} & $\bar{T}=256$ & $\overline{13,6941}$ & 21,5723 & $\overline{24,6090}$ & 2727,0268 & 29,5715 & $=14,3805$ & $\overline{5,3470}$ \\
\hline & $\mathrm{T}=512$ & 13,3904 & 21,4099 & 23,8665 & 26,0190 & 29,0524 & 14,1246 & 5,4351 \\
\hline & $\mathrm{T}=1024$ & 13,1929 & 21,8692 & 24,5980 & 27,8842 & 30,9173 & 14,1352 & 5,7088 \\
\hline & $\mathrm{T}=4096$ & 13,3461 & 21,2934 & 24,2175 & 26,7251 & 29,3990 & 14,0414 & 5,3736 \\
\hline \multicolumn{2}{|c|}{ Referência $\left(\chi_{\nu=14}^{2}\right)$} & 13,3393 & 21,0641 & 23,6848 & 26,1189 & 29,1412 & 14,0000 & 5,2915 \\
\hline \multirow{4}{*}{$\mathrm{J}=3$} & $\mathrm{~T}=256$ & 30,0520 & 42,2974 & $\overline{46,7115}$ & 499,9980 & 54,1043 & 31,2178 & 8,3454 \\
\hline & $\mathrm{T}=512$ & 30,0290 & 41,8309 & 45,7904 & 48,8059 & 53,5229 & 30,9623 & 8,0997 \\
\hline & $\mathrm{T}=1024$ & 29,4354 & 40,8147 & 44,6408 & 48,0286 & 50,9135 & 30,3028 & 7,9237 \\
\hline & $\mathrm{T}=4096$ & 29,6982 & 40,9747 & 44,6088 & 48,4852 & 54,2589 & 30,5523 & 8,0681 \\
\hline \multicolumn{2}{|c|}{ Referência $\left(\chi_{\nu=30}^{2}\right)$} & 29,3360 & 40,2560 & 43,7730 & 46,9792 & 50,8922 & 30,0000 & 7,7460 \\
\hline \multirow{4}{*}{$\mathrm{J}=4$} & $\mathrm{~T}=256$ & 68,8794 & 84,8912 & 88,8051 & 93,7518 & 999,8415 & 68,9673 & 12,0589 \\
\hline & $\mathrm{T}=512$ & 64,5060 & 80,6596 & 85,8852 & 89,3158 & 94,1073 & 64,9175 & 11,9715 \\
\hline & $\mathrm{T}=1024$ & 61,5096 & 77,3247 & 81,6160 & 84,9427 & 91,1403 & 62,3096 & 11,3643 \\
\hline & $\mathrm{T}=4096$ & 61,9107 & 77,4596 & 81,9125 & 85,5765 & 90,0684 & 62,3717 & 11,4675 \\
\hline \multicolumn{2}{|c|}{ Referência $\left(\chi_{\nu=62}^{2}\right)$} & 61,3346 & 76,6302 & 81,3810 & 85,6537 & 90,8015 & 62,0000 & 11,1355 \\
\hline \multirow{4}{*}{$\mathrm{J}=5$} & $\mathrm{~T}=256$ & 165,2770 & 194,7596 & 203,5045 & 208,9855 & 220,0811 & 166,1982 & 22,0120 \\
\hline & $\mathrm{T}=512$ & 135,7438 & 158,7342 & 166,4919 & 172,8982 & 177,8418 & 136,6703 & 17,2265 \\
\hline & $\mathrm{T}=1024$ & 130,1606 & 151,7567 & 158,7198 & 166,2436 & 171,6039 & 130,7952 & 16,1854 \\
\hline & $\mathrm{T}=4096$ & 127,1858 & 148,7893 & 155,6727 & 162,1183 & 169,3733 & 127,2352 & 16,5721 \\
\hline \multicolumn{2}{|c|}{ Referência $\left(\chi_{\nu=126}^{2}\right)$} & 125,3340 & 146,7241 & 153,1979 & 158,9624 & 165,8410 & 126,0000 & 15,8745 \\
\hline
\end{tabular}


Com base nos quantis apresentados nas Tabelas 5.1, 5.2 e 5.3, parece razoável assumir que a estatística $R V_{C V T}^{(J)}$ segue assintoticamente uma distribuição $\chi_{\nu}^{2}$. Assim, podemos fazer testes para determinar se um conjunto de dados apresenta cointegração que varia com o tempo, dado um nível de significância fixado.

A Tabela 5.4, por sua vez, apresenta o poder empírico do teste de razão de verossimilhanças $R V_{C V T}^{(J)}$, calculado via simulações para diferentes valores de $T$, utilizando ondaleta Haar e nível de significância de 5\%. Pela tabela, podemos notar que o poder fica cada vez mais próximo de 1 à medida que aumentamos o tamanho da amostra. Como esperado, as exceções são as combinações de $\beta_{21}$ e $\beta_{22}$ que correspondem à hipótese nula $\left(\beta_{21}=-1\right.$ e $\left.\beta_{22}=-1\right)$, caso em que obtemos o tamanho do teste ou quando estamos muito próximas da hipótese nula, como $\left(\beta_{21}=-1, \beta_{22}=-1,0005\right)$ e $\left(\beta_{21}=-1, \beta_{22}=-0,9995\right)$.

Tabela 5.4: Poder empírico do teste de razão de verossimilhanças $R V_{C V T}^{(J)}$.

\begin{tabular}{|cc|cccc|}
\hline \multicolumn{2}{|c|}{ Poder } & \multicolumn{4}{c|}{$\mathrm{T}$} \\
\hline \multicolumn{2}{|c|}{ Valores dos parâmetros } & 256 & 512 & 1024 & 10240 \\
\hline$\beta_{21}=-1$, & $\beta_{22}<-1,2500$ & 1,000 & 1,000 & 1,000 & 1,000 \\
$\beta_{21}=-1$, & $\beta_{22}=-1,2500$ & 0,990 & 1,000 & 1,000 & 1,000 \\
$\beta_{21}=-1$, & $\beta_{22}=-1,1500$ & 0,989 & 1,000 & 1,000 & 1,000 \\
$\beta_{21}=-1$, & $\beta_{22}=-1,0500$ & 0,616 & 0,942 & 0,999 & 1,000 \\
$\beta_{21}=-1$, & $\beta_{22}=-1,0050$ & 0,052 & 0,100 & 0,198 & 1,000 \\
$\beta_{21}=-1$, & $\beta_{22}=-1,0005$ & 0,046 & 0,043 & 0,048 & 0,219 \\
$\beta_{21}=-\mathbf{1}$, & $\beta_{22}=-\mathbf{1 , 0 0 0 0}$ & $\mathbf{0 , 0 5 0}$ & $\mathbf{0 , 0 5 0}$ & $\mathbf{0 , 0 5 0}$ & $\mathbf{0 , 0 5 0}$ \\
$\beta_{21}=-1$ & $\beta_{22}=-0,9995$ & 0,040 & 0,067 & 0,053 & 0,234 \\
$\beta_{21}=-1$ & $\beta_{22}=-0,9950$ & 0,067 & 0,086 & 0,198 & 1,000 \\
$\beta_{21}=-1$ & $\beta_{22}=-0,9500$ & 0,612 & 0,917 & 1,000 & 1,000 \\
$\beta_{21}=-1$ & $\beta_{22}=-0,8500$ & 0,992 & 1,000 & 1,000 & 1,000 \\
$\beta_{21}=-1$ & $\beta_{22}=-0,7500$ & 0,999 & 1,000 & 1,000 & 1,000 \\
$\beta_{21}=-1$ & $\beta_{22}>-0,7500$ & 1,000 & 1,000 & 1,000 & 1,000 \\
\hline
\end{tabular}


A seguir, avaliamos a distribuição dos estimadores dos parâmetros do modelo de cointegração variando com tempo, definido em 4.3. Consideramos simulações de séries bivariadas $(n=2)$, com uma tendência comum $(r=1)$ e com $T=1024$ observações. O modelo cointegrado (normalizado) na forma de correção de erros pode ser escrito como:

$$
\left[\begin{array}{l}
\Delta Y_{1 t} \\
\Delta Y_{2 t}
\end{array}\right]=\left[\begin{array}{r}
-1 \\
0
\end{array}\right]\left[\begin{array}{ll}
1 & \beta_{2 t}
\end{array}\right]\left[\begin{array}{l}
Y_{1, t-1} \\
Y_{2, t-1}
\end{array}\right]+\boldsymbol{\Gamma} \Delta X+\left[\begin{array}{l}
u_{1 t} \\
u_{2 t}
\end{array}\right]
$$

com

$$
\Delta Y t=\left[\begin{array}{l}
\Delta Y_{1 t} \\
\Delta Y_{2 t}
\end{array}\right], \boldsymbol{\alpha}=\left[\begin{array}{r}
-1 \\
0
\end{array}\right], \boldsymbol{\beta}_{t}=\left[\begin{array}{ll}
1 & \beta_{2 t}
\end{array}\right], Y_{t-1}=\left[\begin{array}{l}
Y_{1, t-1} \\
Y_{2, t-1}
\end{array}\right] \text { e } u_{t}=\left[\begin{array}{l}
u_{1 t} \\
u_{2 t}
\end{array}\right]
$$

Desconsiderando o termo $\Gamma \Delta X$ (sem defasagem), o modelo cointegrado definido em 5.1 pode ser simulado de acordo com a expressão 5.3, tomando $u_{1 t} \sim$ i.i.d. $\mathrm{N}(0,1)$ e $u_{2 t} \sim$ i.i.d. $\mathrm{N}(0,1)$ :

$$
\left\{\begin{array}{l}
Y_{1 t}=-\beta_{2 t} Y_{2, t-1}+u_{1 t} \\
Y_{2 t}=Y_{2, t-1}+u_{2 t}
\end{array} .\right.
$$

Consideramos cinco exemplos simulados sem defasagem e um com defasagem. Supomos que $\boldsymbol{\beta}_{t}$ está normalizado conforme a Equação 4.54 para garantir a identificabilidade dos estimadores. Em cada exemplo, apresentamos as séries cointegradas simuladas, a componente $\hat{\beta}_{2 t}$ do vetor de cointegração normalizado estimado $\hat{\boldsymbol{\beta}}_{t}^{*}=\left[1, \hat{\beta}_{2 t}^{*}\right]^{\prime}$, para $t=1, \ldots, T$, além dos resíduos de cointegração estimados $\hat{\boldsymbol{\beta}}_{t}^{*} Y_{t-1}$. Também são apresentadas estimativas, médias, desvios padrão e histogramas das estimativas obtidas via simulações bootstrap. Em cada exemplo, apresentamos os resultados para a hipótese nula (cointegração constante) e para a hipótese alternativa (cointegração variando com o tempo).

\subsection{Exemplos sem defasagem}

\subsubsection{Primeiro exemplo: Inversão de sinal ao longo do tempo com Haar}

Desejamos testar a capacidade do modelo em estimar o vetor de cointegração normalizado $\boldsymbol{\beta}_{t}^{*}$, que assume dois regimes ao longo do tempo para $T=1024$. Para isso, consideramos o vetor de cointegração teórico:

$$
\boldsymbol{\beta}_{t}^{*}=\left[\begin{array}{c}
\beta_{1 t}^{*} \\
\beta_{2 t}^{*}
\end{array}\right]=\left\{\begin{array}{rr}
{\left[\begin{array}{ll}
1 & -1
\end{array}\right]^{\prime}, \text { se } \quad 1 \leq t \leq T / 2} \\
{\left[\begin{array}{ll}
1 & 1
\end{array}\right]^{\prime}, \text { se } \quad T / 2+1 \leq t \leq T}
\end{array} .\right.
$$

Utilizando a expressão 4.1 e $J=0$, podemos escrever o vetor $\boldsymbol{\beta}_{t}^{*}$ por meio da seguinte expressão: 


$$
\boldsymbol{\beta}_{t}^{*}=\phi(t) \mathbf{c}^{*}+\psi_{0,0}(t) \mathbf{d}_{0,0}^{*}
$$

Pela Equação 4.2 temos que $\mathbf{c}^{*}=\left[\begin{array}{lll}c_{0,0}^{*(1,1)} & c_{0,0}^{*(2,1)}\end{array}\right]^{\prime}$ e $\mathbf{d}_{0,0}^{*}=\left[\begin{array}{ll}d_{0,0}^{*(1,1)} & d_{0,0}^{*(2,1)}\end{array}\right]^{\prime}$ são matrizes $(n=2 \times r=1)$, cujos elementos são estimados por $\hat{\boldsymbol{W}}_{J}^{*}$ (Equação 4.63). Além disso, $\phi(t)$ e $\psi_{0,0}(t)$ são funções da base de ondaletas Haar.

Apresentamos na Figura 5.1 (a) os gráficos das séries cointegradas simuladas $Y_{1 t}$ e $Y_{2 t}$. Para estas séries, apresentamos em 5.1 (b) o segundo componente dos vetores de cointegração teórico $\left(\beta_{2 t}^{*}\right)$ e estimado $\left(\hat{\beta}_{2 t}^{*}\right)$. Pela figura, é possível notar que $\hat{\beta}_{2 t}^{*}$ está muito próximo de $\beta_{2 t}^{*}$, sendo que ambos estão dentro das bandas de confiança de $95 \%$. Além disso, apresentamos as estimativas dos parâmetros do modelo, juntamente com os valores teóricos em parênteses:

$$
\begin{gathered}
\hat{\boldsymbol{\beta}}_{t}^{*}=\left[\begin{array}{cccccc}
1 & \ldots & 1 & 1 & \ldots & 1 \\
-1,0002 & \ldots & -1,0002 & 1,0024 & \ldots & 1,0024 \\
(-1,0000) & & (-1,0000) & (1,0000) & & (1,0000)
\end{array}\right], \hat{\boldsymbol{\alpha}}^{*}=\left[\begin{array}{c}
-0,9963 \\
(-1,0000) \\
-0,0353 \\
(0,0000)
\end{array}\right], \\
\hat{\boldsymbol{c}}^{*}=\left[\begin{array}{c}
1,0000 \\
0,0009 \\
(0,0000)
\end{array}\right], \hat{\boldsymbol{d}}^{*}=\left[\begin{array}{c}
0,0000 \\
-0,9985 \\
(-1,0000)
\end{array}\right] \text { e } \hat{\boldsymbol{\Sigma}}_{u}^{*}=\left[\begin{array}{cc}
0,9964 & -0,0313 \\
(1,0000) & (0,0000) \\
-0,0313 & 0,9930 \\
(0,0000) & (1,0000)
\end{array}\right] .
\end{gathered}
$$

Apresentamos na Figura 5.1 (c) os resíduos de cointegração teóricos $\left(\boldsymbol{\beta}_{t}^{*^{\prime}} Y_{t-1}\right)$ e estima$\operatorname{dos}\left(\hat{\boldsymbol{\beta}}_{t}^{*^{\prime}} Y_{t-1}\right)$. Verificamos que $\hat{\boldsymbol{\beta}}_{t}^{*} Y_{t-1}$ correspondem a um processo estacionário (valor- $p$ $<0,01$ para o teste DFA - Dickey-Fuller Aumentado). 
(a)

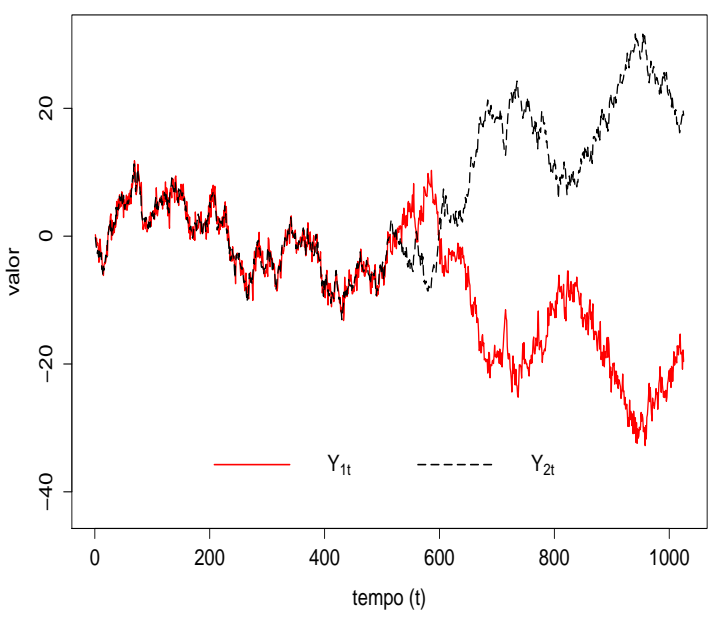

(b)

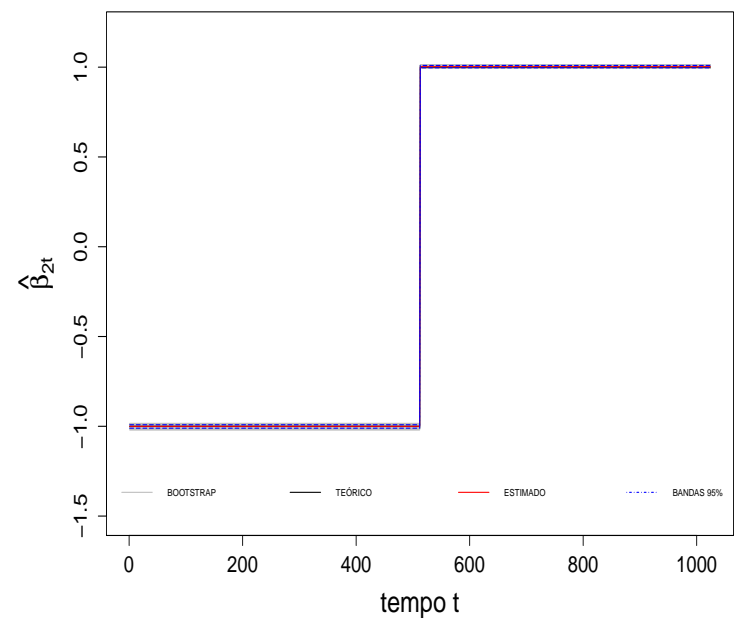

(c)

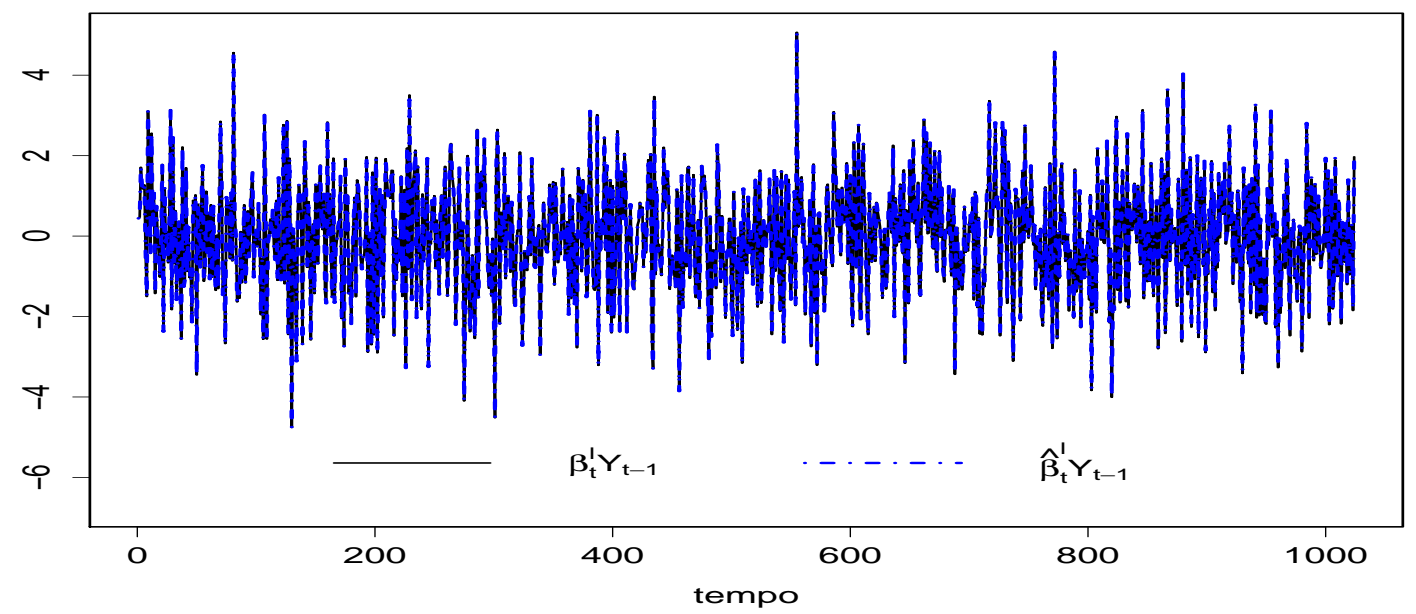

Figura 5.1: Primeiro exemplo: (a) Séries cointegradas simuladas $Y_{1 t}$ e $Y_{2 t}$, com $T=1024$, (b)

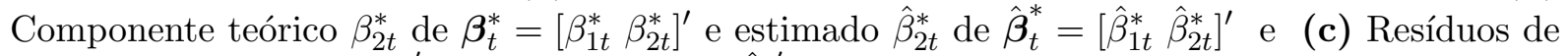
cointegração teóricos $\left(\beta_{t}^{*^{\prime}} Y_{t-1}\right)$ e estimados $\left(\hat{\beta}_{t}^{*^{\prime}} Y_{t-1}\right)$.

Apresentamos na Figura 5.2 os resíduos $\hat{u}_{1 t}$ e $\hat{u}_{2 t}$ do modelo. Ao aplicarmos o teste de Shapiro-Wilk individualmente a $\hat{u}_{1 t}$ e $\hat{u}_{2 t}$, verificamos que eles parecem ter uma distribuição normal (valores- $p$ iguais a 0,7846 e 0,5273 respectivamente). Conforme esperado, validamos a suposição de normalidade do modelo, visto que o modelo foi simulado sob esta condição. Tal fato é confirmado pelos QQ-plots apresentados em 5.2. Além disso, os resíduos parecem ser não-correlacionados, conforme sugerem os correlogramas apresentados na mesma figura. 

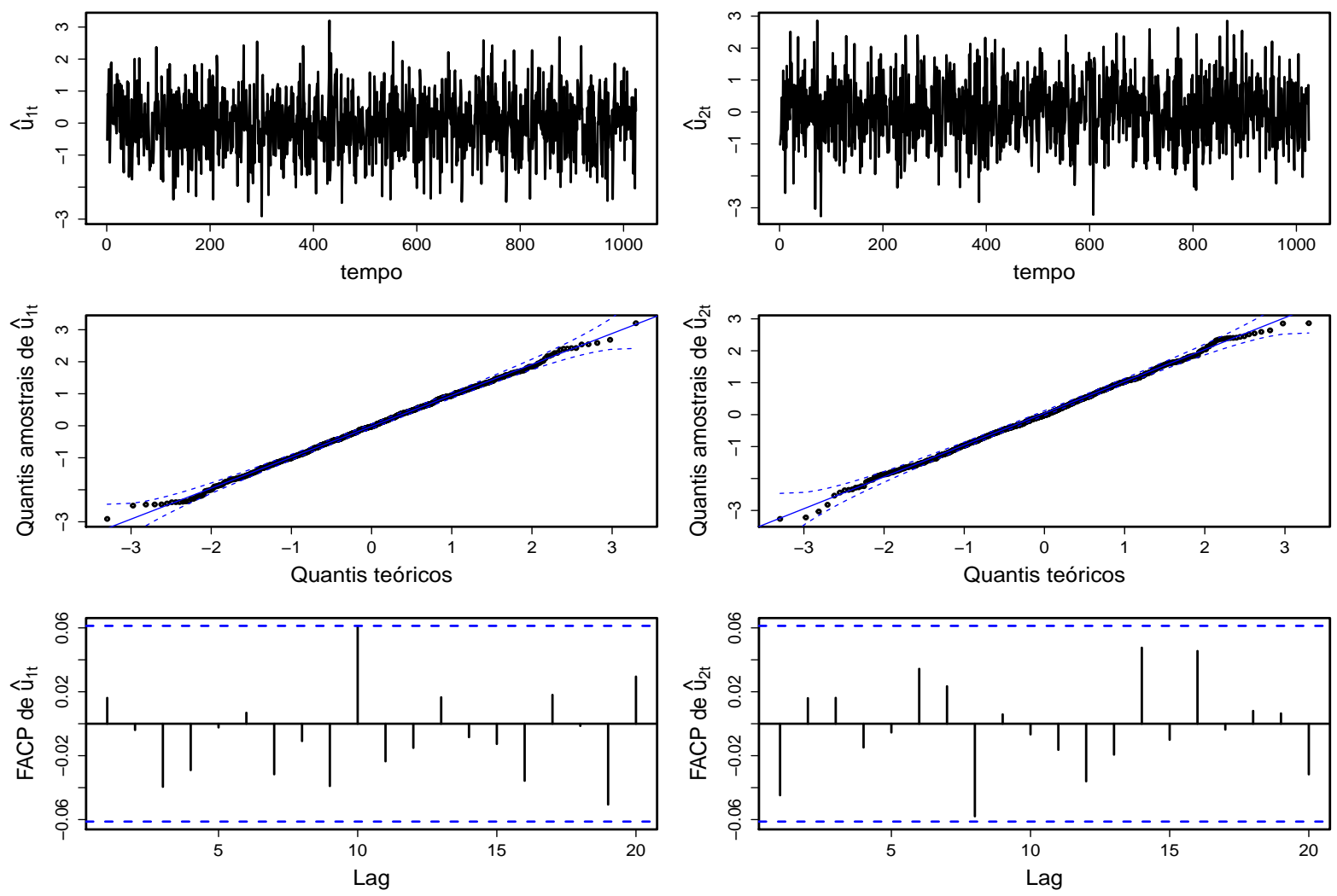

Figura 5.2: Primeiro exemplo: Resíduos estimados pelo modelo, quantis amostrais e função de autocorrelação parcial (FACP) de $\hat{u}_{t}=\left[\hat{u}_{1 t}, \hat{u}_{2 t}\right]^{\prime}$.

Por meio do valor- $p$ bootstrap apresentado na Tabela 5.5, existem evidências que nos levam a rejeitar a hipótese de cointegração constante ao longo do tempo.

Tabela 5.5: Ex.01: Estimativa e medidas bootstrap ${ }^{\star}$ sob H0 (vetor de cointegração constante).

\begin{tabular}{c|ccccc}
\hline Parâmetro & Estimativa & Intervalo de confiança ${ }^{\star}$ & Média $^{*}$ & Erro Padrão $^{*}$ & valor- $p^{*}$ \\
\hline$R V_{C V T}^{(J)}(\mathrm{H} 0)$ & 1283,96 & {$[0,0493 ; 6,9310]$} & 1,9692 & 2,0206 & 0,0000 \\
\hline
\end{tabular}

A Tabela 5.6 apresenta as estimativas dos parâmetros, bem como medidas bootstrap de interesse. Podemos notar que as estimativas e as médias bootstrap estão próximas dos valores teóricos dos parâmetros.

Tabela 5.6: Ex.01: Estimativas e medidas bootstrap* sob HA (inversão de sinal).

\begin{tabular}{c|ccccc}
\hline Parâmetro & Teórico & Estimativa & Intervalo de confiança & Média $^{\star}$ & Erro Padrão $^{\star}$ \\
\hline$R V_{C V T}^{(J)}(\mathrm{HA})$ & - & 1283,96 & {$[1059,66 ; 2181,40]$} & 1347,32 & 291,32 \\
$\alpha_{11}^{*}$ & $-1,000$ & $-0,9963$ & {$[-1,0413 ;-0,9669]$} & $-1,0006$ & 0,0195 \\
$\alpha_{21}^{*}$ & 0,0000 & $-0,0353$ & {$[-0,0397 ; 0,0371]$} & $-0,0006$ & 0,0200 \\
$\sigma_{11}^{*}$ & 1,0000 & 0,9964 & {$[0,9046 ; 1,0755]$} & 0,9920 & 0,0434 \\
$\sigma_{12}^{*}=\sigma_{21}^{*}$ & 0,0000 & $-0,0313$ & {$[-0,0612 ; 0,0559]$} & $-0,0004$ & 0,0301 \\
$\sigma_{22}^{*}$ & 1,0000 & 0,9930 & {$[0,9103 ; 1,0703]$} & 0,9912 & 0,0412 \\
$\hat{c}_{0,0}^{*(2,1)}$ & 0,0000 & 0,0009 & {$[-0,0059 ; 0,0072]$} & 0,0008 & 0,0032 \\
$\hat{d}_{0,0}^{*(2,1)}$ & $-1,0000$ & $-0,9985$ & {$[-1,0047 ;-0,9918]$} & $-0,9985$ & 0,0034 \\
\hline
\end{tabular}


Apresentamos nas Figuras 5.3, 5.4, 5.5 e 5.6 os histogramas bootstrap dos parâmetros do modelo. Podemos notar em todas as figuras que os valores teóricos dos parâmetros estão dentro dos intervalos de confiança bootstrap de 95\%. Pelas figuras ainda é possível comparar a estimativa de cada parâmetro do modelo com sua respectiva média boostrap. Apesar de estarem dentro dos intervalos de confiança bootstrap, percebemos um pequeno desvio entre as estimativas e médias bootstrap para o parâmetro $\hat{\alpha}_{21}^{*}$.

(a)

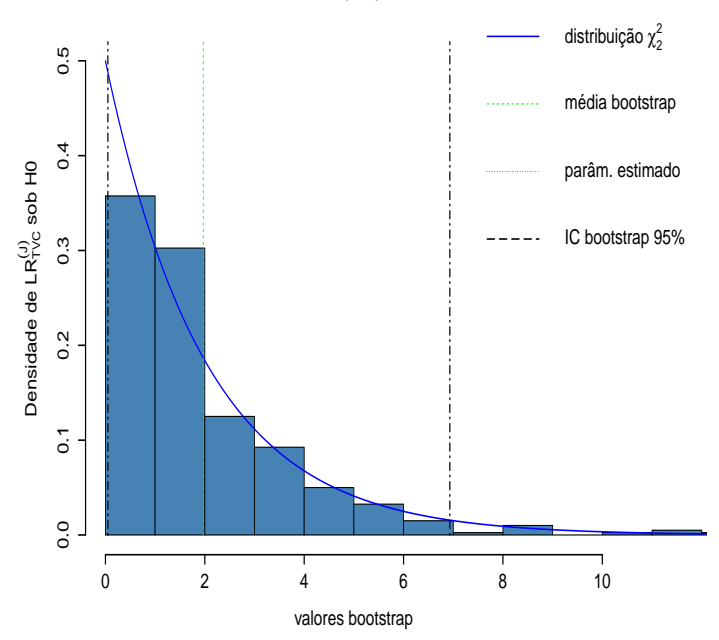

(b)

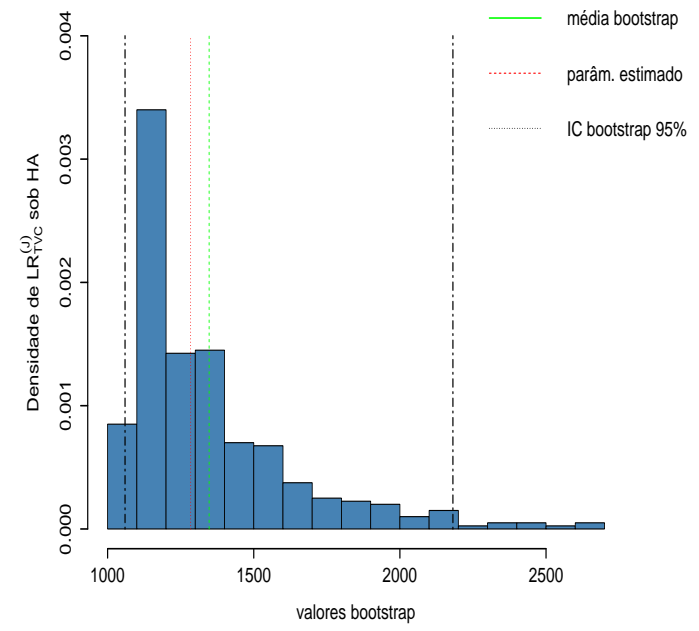

Figura 5.3: Primeiro exemplo: Histograma, densidade e quantis bootstrap 2,5\% e 97, $5 \%$ para $R V_{C V T}^{(J)}$ sob H0 (vetor de cointegração constante) (a) e sob HA (inversão de sinal ao longo do tempo) (b).

(a)

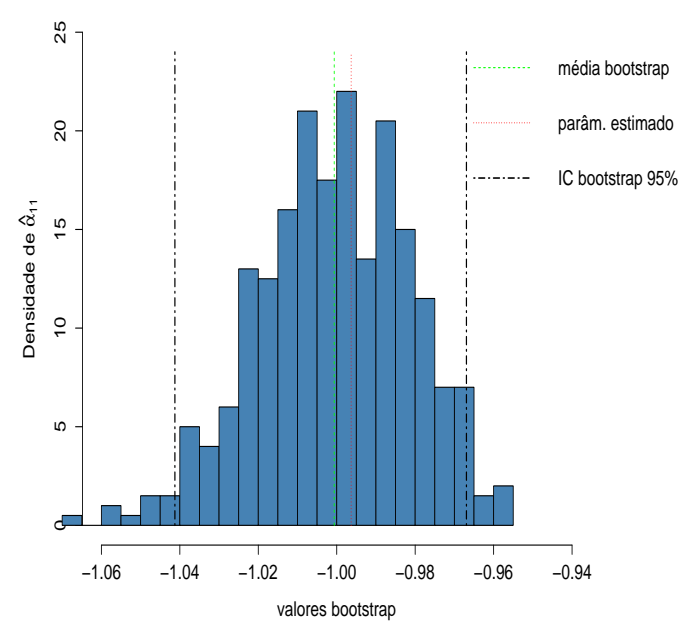

(b)

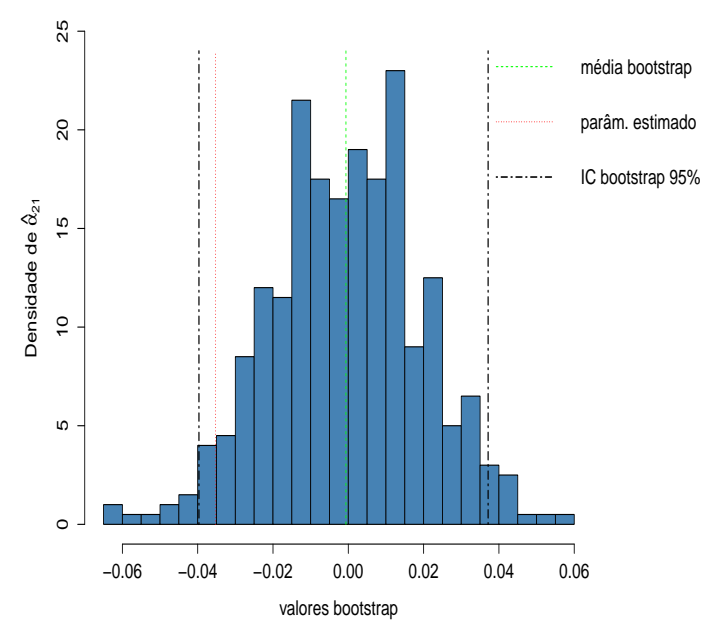

Figura 5.4: Primeiro exemplo: Histograma, densidade e quantis bootstrap 2, 5\% e 97, $5 \%$ sob HA

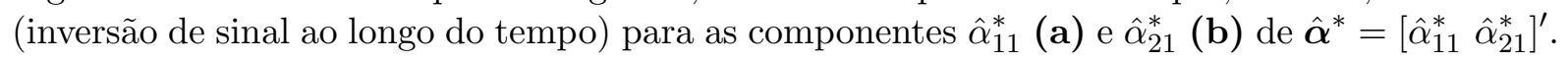


(a)

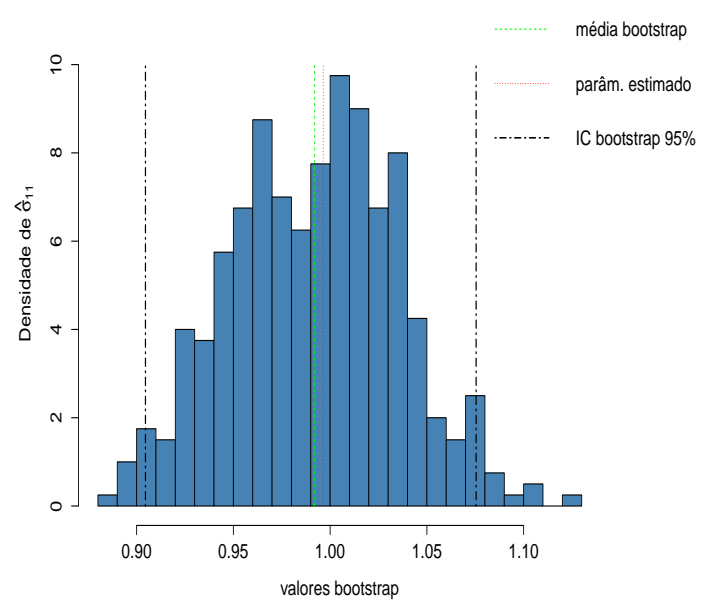

(b)

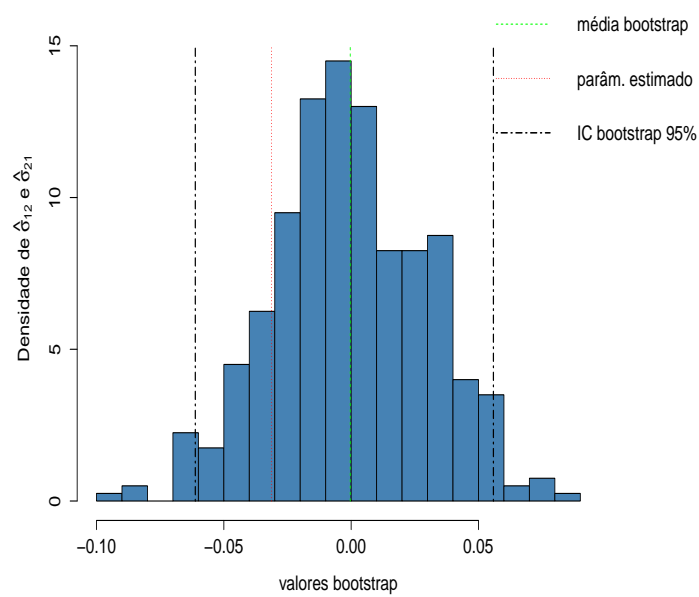

(c)

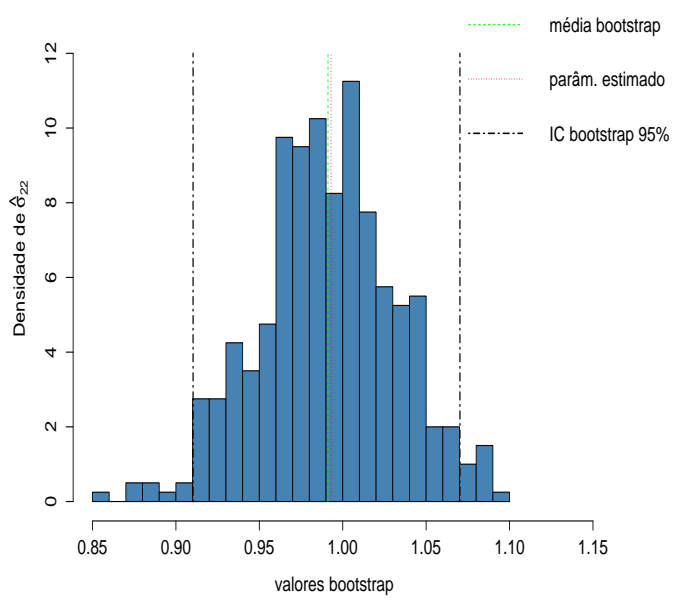

Figura 5.5: Primeiro exemplo: Histograma, densidade e quantis bootstrap 2, 5\% e 97, 5\% sob HA (inversão de sinal ao longo do tempo) para a componente $\hat{\sigma}_{11}^{*}$ (a), componentes $\hat{\sigma}_{12}^{*}$ e $\hat{\sigma}_{21}^{*}$ (b) e componente $\hat{\sigma}_{22}^{*}$ (c) de $\hat{\mathbf{\Sigma}}^{*}$.

(a)

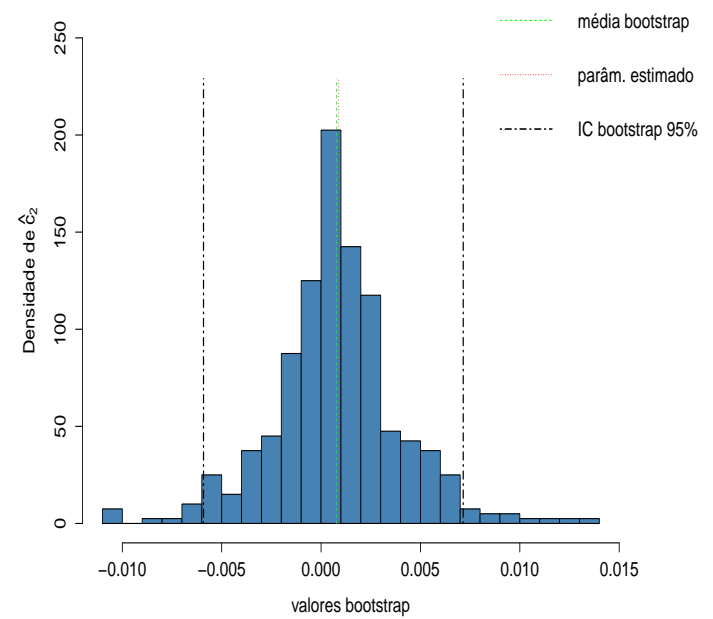

(b)

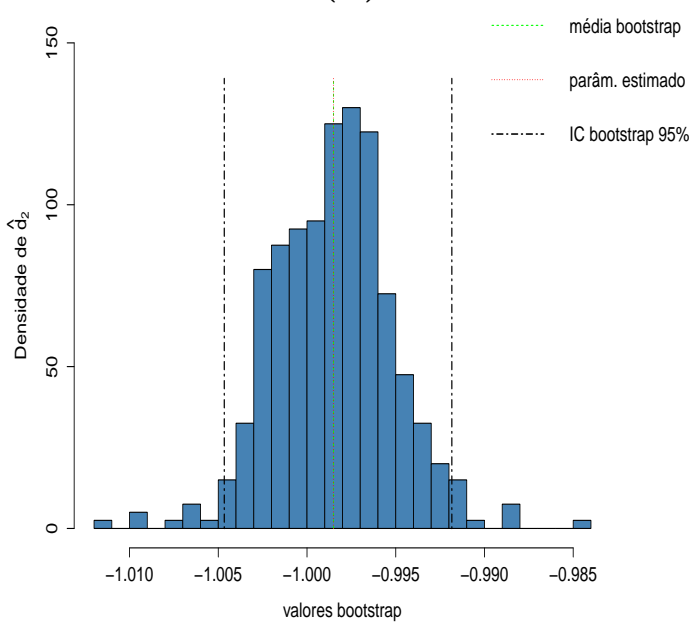

Figura 5.6: Primeiro exemplo: Histograma, densidade e quantis bootstrap 2,5\% e 97,5\% sob HA (inversão de sinal ao longo do tempo) para as componentes $\hat{c}_{0,0}^{*(2,1)}$ (a) e $\hat{d}_{0,0}^{*(2,1)}$ (b) de

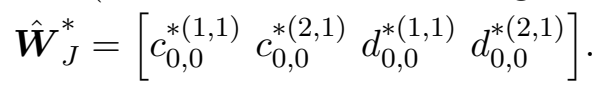


Por meio da Tabela 5.7, desejamos avaliar a convergência numérica dos estimadores normalizados $\boldsymbol{\alpha}^{*}, \boldsymbol{\beta}_{t}^{*}, \boldsymbol{\Sigma}_{u}^{*}$ e $\hat{\boldsymbol{W}}_{J}^{*}$ do modelo. Os resultados foram obtidos por meio de $N=1000$ processos cointegrados simulados, considerando séries com tamanhos $T=$ 256, 512, 1024 e 10240. Para $T$, calculamos os quantis, as médias e desvios padrão das estimativas dos parâmetros. Nas simulações, consideramos os parâmetros normalizados:

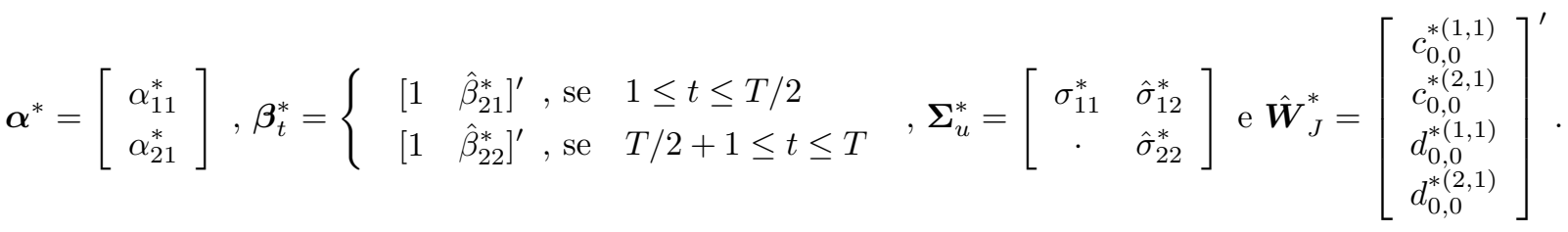

Tabela 5.7: Ex.01: Estatísticas das estimativas dos parâmetros normalizados, obtidas por meio de 1000 processos cointegrados bivariados simulados (inversão de sinal ao longo do tempo).

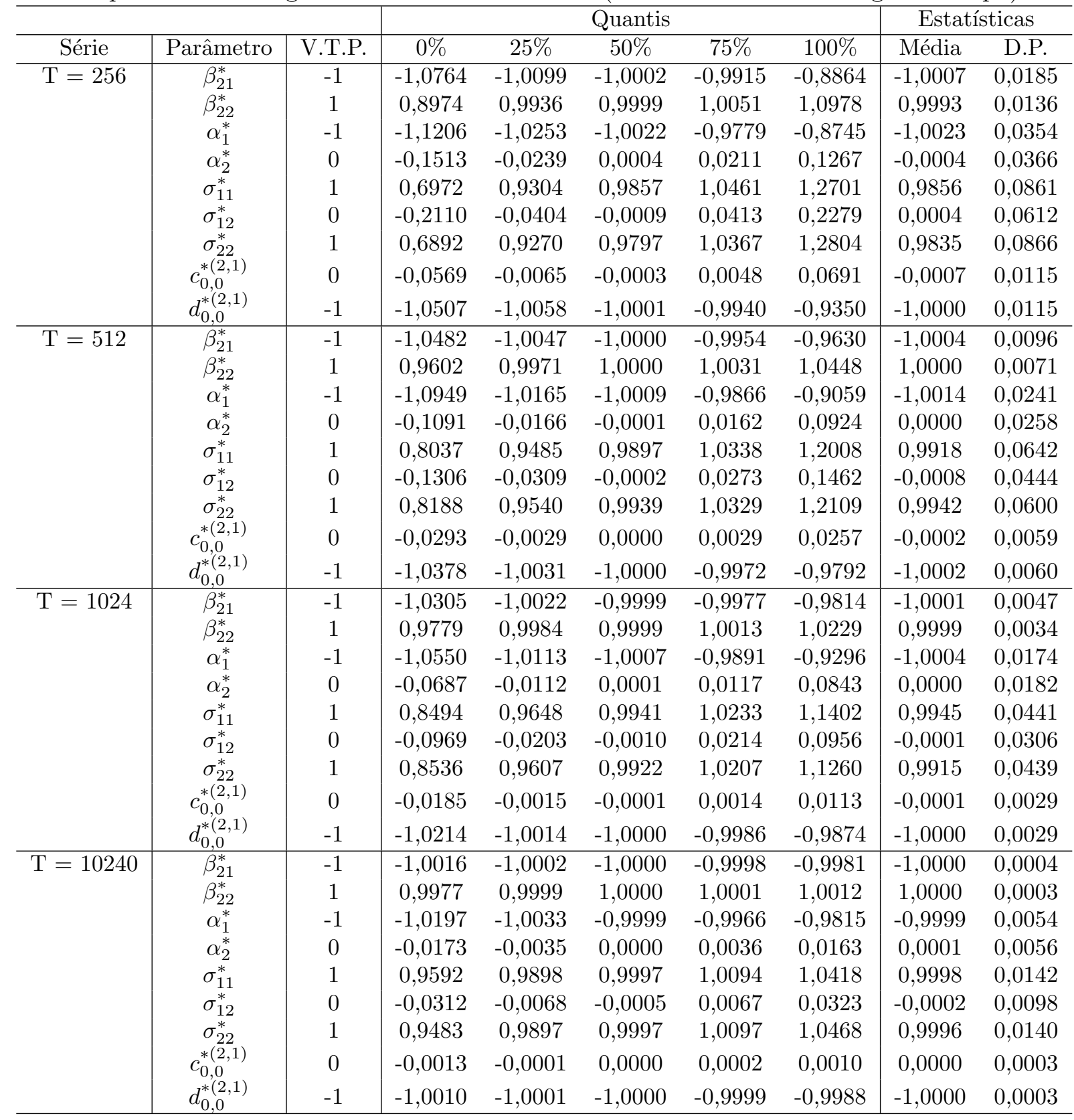

V.T.P = Valor Teórico do Parâmetro e D.P. = Desvio Padrão. 
Como os parâmetros do modelo estão normalizados, conforme apresentado na Equação 4.58 , temos que $c_{0,0}^{*(1,1)}=1$ e $d_{0,0}^{*(1,1)}=0$, independente do tamanho $T$ das séries considerado nas simulações. Por esta razão, apresentamos na tabela 5.7 apenas os termos $c_{0,0}^{*(2,1)}$ e $d_{0,0}^{*(2,1)}$ de $\hat{\boldsymbol{W}}_{J}^{*}$.

Pela tabela 5.7, podemos notar que à medida que aumentamos o tamanho das séries, os valores médios e medianos dos parâmetros convergem para os valores teóricos. Além disso, observamos que o desvio padrão diminui para todas as estimativas dos parâmetros à medida que aumentamos o tamanho das séries cointegradas simuladas.

\subsubsection{Segundo exemplo: Vetor de cointegração constante com Haar}

Neste exemplo, o nosso objetivo é verificar se é possível estimar os parâmetros do modelo, sem que hajam alterações em $\boldsymbol{\beta}_{t}^{*}$ ao longo do tempo, com $T=1024$. Desejamos avaliar também a distribuição da estatística $R V_{C V T}^{(J)}$ sob H0. Consideramos o vetor de cointegração teórico:

$$
\boldsymbol{\beta}_{t}^{*}=\boldsymbol{\beta}^{*}=\left[\begin{array}{c}
\beta_{1 t}^{*} \\
\beta_{2 t}^{*}
\end{array}\right]=\left[\begin{array}{c}
1 \\
-1
\end{array}\right] \text {, para } 1 \leq t \leq T .
$$

Utilizando a expressão 4.1 e $J=0$, podemos escrever o vetor $\boldsymbol{\beta}_{t}^{*}$ por meio da seguinte expressão:

$$
\boldsymbol{\beta}_{t}^{*}=\phi(t) \mathbf{c}^{*}+\psi_{0,0}(t) \mathbf{d}_{0,0}^{*} .
$$

Por 4.2 temos que $\mathbf{c}^{*}=\left[\begin{array}{lll}c_{0,0}^{*(1,1)} & c_{0,0}^{*(2,1)}\end{array}\right]^{\prime}$ e $\mathbf{d}_{0,0}^{*}=\left[\begin{array}{ll}d_{0,0}^{*(1,1)} & d_{0,0}^{*(2,1)}\end{array}\right]^{\prime}$, cujos elementos são estimados por $\hat{\boldsymbol{W}}_{J}^{*}$ de 4.63. Além disso, $\phi(t)$ e $\psi_{0,0}(t)$ são funções da base de ondaletas Haar.

Apresentamos na Figura 5.7 (a) os gráficos das séries cointegradas simuladas $Y_{1 t}$ e $Y_{2 t}$. Para estas séries, apresentamos em 5.7 (b) o segundo componente dos vetores de cointegração teórico $\left(\beta_{2 t}^{*}\right)$ e estimado $\left(\hat{\beta}_{2 t}^{*}\right)$. Pela figura, é possível notar que $\hat{\beta}_{2 t}^{*}$ está muito próximo de $\beta_{2 t}^{*}$, sendo que ambos estão dentro das bandas de confiança de $95 \%$. Além disso, apresentamos as estimativas dos parâmetros do modelo, juntamente com os valores teóricos em parênteses:

$$
\begin{gathered}
\hat{\boldsymbol{\beta}}_{t}^{*}=\left[\begin{array}{cccccc}
1 & \ldots & 1 & 1 & \ldots & 1 \\
-1,0003 & \ldots & \underset{(-1,0003}{-1,0000)} & \underset{(-1,0000)}{-0,9973} & \ldots & \underset{(-1,0000)}{-1,000)}
\end{array}\right], \hat{\boldsymbol{\alpha}}^{*}=\left[\begin{array}{c}
-1,0147 \\
(-1,0000) \\
0,0226 \\
(0,0000)
\end{array}\right], \\
\hat{\boldsymbol{c}}^{*}=\left[\begin{array}{c}
1,0000 \\
-0,9988 \\
(-1,0000)
\end{array}\right], \hat{\boldsymbol{d}}^{*}=\left[\begin{array}{c}
0,0000 \\
-0.0014 \\
(0,0000)
\end{array}\right] \text { e } \hat{\boldsymbol{\Sigma}}_{u}^{*}=\left[\begin{array}{cc}
0,9360 & -0,0187 \\
(1,0000) & (0,0000) \\
-0,0187 & 0,9841 \\
(0,0000) & (1,0000)
\end{array}\right] .
\end{gathered}
$$


Apresentamos na Figura 5.7 (c) os resíduos de cointegração teóricos $\left(\boldsymbol{\beta}_{t}^{*^{\prime}} Y_{t-1}\right)$ e estima$\operatorname{dos}\left(\hat{\boldsymbol{\beta}}_{t}^{*^{\prime}} Y_{t-1}\right)$. Verificamos que $\hat{\boldsymbol{\beta}}_{t}^{*} Y_{t-1}$ correspondem a um processo estacionário (valor- $p$ $<0,01$ para o teste DFA - Dickey-Fuller Aumentado).

(a)

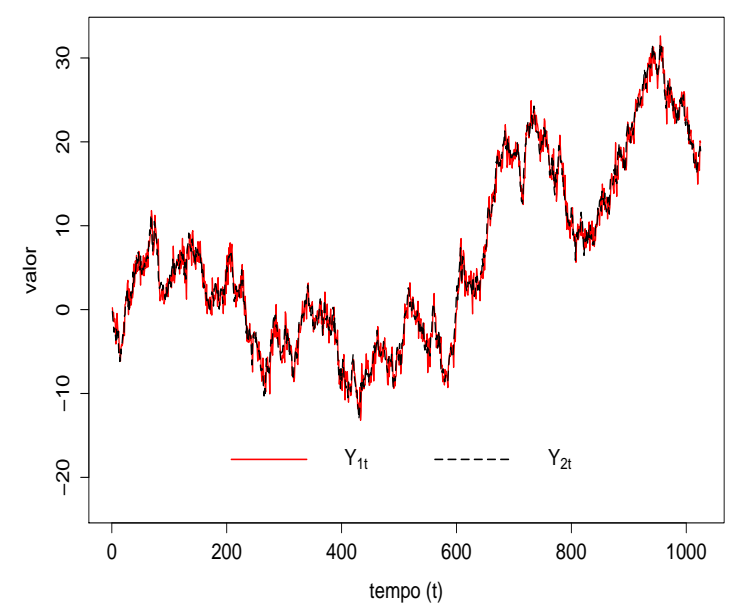

(b)

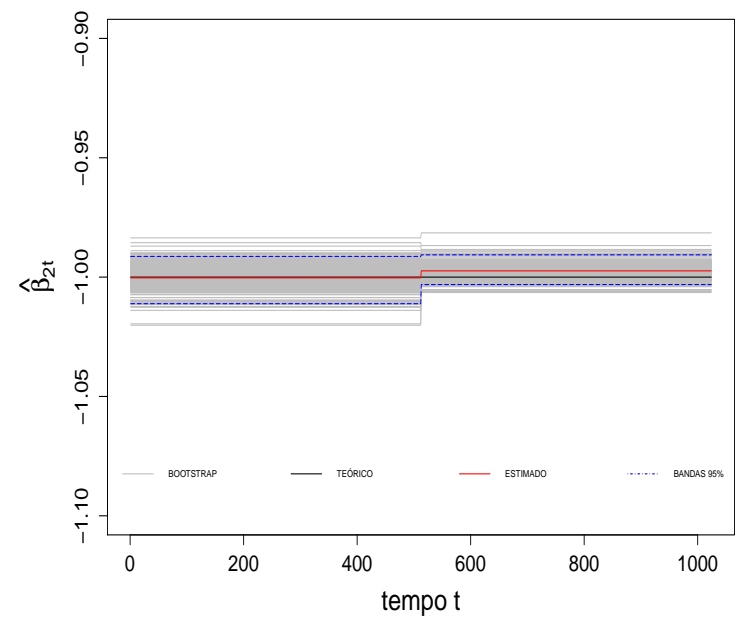

(c)

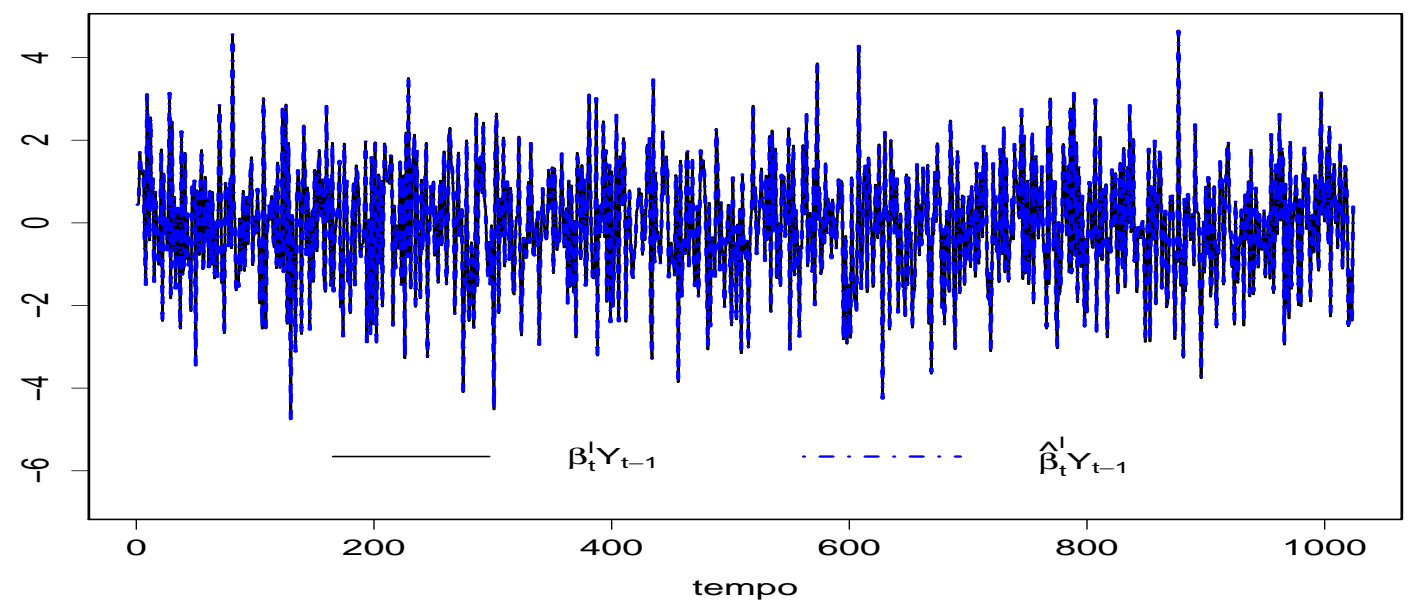

Figura 5.7: Segundo exemplo: (a) Séries cointegradas simuladas $Y_{1 t}$ e $Y_{2 t}$, com $T=1024$, (b) Componente teórico $\beta_{2 t}^{*}$ de $\boldsymbol{\beta}_{t}^{*}=\left[\beta_{1 t}^{*} \beta_{2 t}^{*}\right]^{\prime}$ e estimado $\hat{\beta}_{2 t}^{*}$ de $\hat{\boldsymbol{\beta}}_{t}^{*}=\left[\hat{\beta}_{1 t}^{*} \hat{\beta}_{2 t}^{*}\right]^{\prime}$ e (c) Resíduos de cointegração teóricos $\left(\beta_{t}^{*^{\prime}} Y_{t-1}\right)$ e estimados $\left(\hat{\beta}_{t}^{*^{\prime}} Y_{t-1}\right)$.

Apresentamos na Figura 5.8 os resíduos $\hat{u}_{1 t}$ e $\hat{u}_{2 t}$ do modelo. Ao aplicarmos o teste de Shapiro-Wilk individualmente a $\hat{u}_{1 t}$ e $\hat{u}_{2 t}$, verificamos que eles parecem ter uma distribuição normal (valores-p iguais a 0,8597 e 0,5514 respectivamente). Conforme esperado, validamos a suposição de normalidade do modelo, visto que o modelo foi simulado sob esta condição. Tal fato é confirmado pelos QQ-plots apresentados em 5.8. Além disso, os resíduos parecem ser não-correlacionados, conforme sugerem os correlogramas apresentados na mesma figura. 

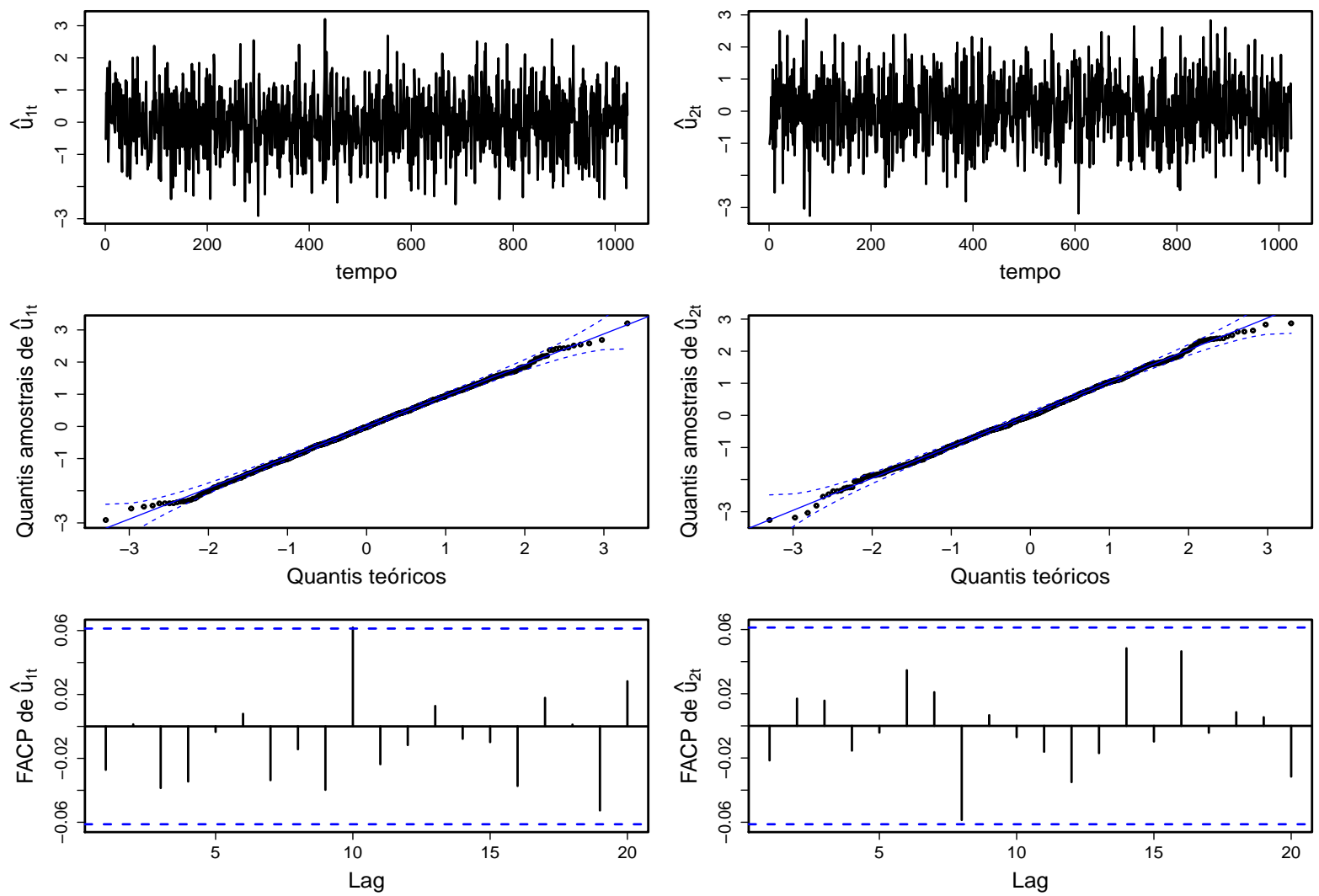

Figura 5.8: Segundo exemplo: Resíduos estimados pelo modelo, quantis amostrais e função de autocorrelação parcial (FACP) de $\hat{u}_{t}=\left[\hat{u}_{1 t}, \hat{u}_{2 t}\right]^{\prime}$.

Por meio do valor- $p$ bootstrap apresentado na Tabela 5.8, existem evidências que nos leva a não rejeitar a hipótese de cointegração constante ao longo do tempo.

Tabela 5.8: Ex.02: Estimativa e medidas bootstrap* sob H0 (vetor de cointegração constante).

\begin{tabular}{c|ccccc}
\hline Parâmetro & Estimativa & Intervalo de confiança ${ }^{\star}$ & Média $^{*}$ & Erro Padrão $^{\star}$ & valor- $p^{*}$ \\
\hline$R V_{C V T}^{(J)}(\mathrm{H} 0)$ & 1,3706 & {$[0,0363 ; 6,7297]$} & 1,9245 & 1,8664 & 0,5100 \\
\hline
\end{tabular}

A Tabela 5.9 apresenta as estimativas dos parâmetros, bem como medidas bootstrap de interesse. Podemos notar que as estimativas e as médias bootstrap estão próximas dos valores teóricos dos parâmetros.

Tabela 5.9: Ex.02: Estimativas e medidas bootstrap ${ }^{\star}$ sob H0 (vetor de cointegração constante).

\begin{tabular}{c|ccccc}
\hline Parâmetro & Teórico & Estimativa & Intervalo de confiança & Média $^{\star}$ & Erro Padrão $^{\star}$ \\
\hline$\alpha_{11}^{*}$ & $-1,000$ & $-1,0147$ & {$[-1,0458 ;-0,9579]$} & $-1,0012$ & 0,0220 \\
$\alpha_{21}^{*}$ & 0,0000 & 0,0226 & {$[-0,0395 ; 0,0394]$} & 0,0020 & 0,0218 \\
$\sigma_{11}^{*}$ & 1,0000 & 0,9361 & {$[0,8581 ; 1,0086]$} & 0,9345 & 0,0400 \\
$\sigma_{12}^{*}=\sigma_{21}^{*}$ & 0,0000 & $-0,0187$ & {$[-0,0615 ; 0,0577]$} & $-0,0025$ & 0,0300 \\
$\sigma_{22}^{*}$ & 1,0000 & 0,9841 & {$[0,9004 ; 1,0584]$} & 0,9790 & 0,0409 \\
$\hat{c}_{0,0}^{*(2,1)}$ & $-1,0000$ & $-0,9988$ & {$[-1,0072 ;-0,9939]$} & $-0,9992$ & 0,0029 \\
$\hat{d}_{0,0}^{*(2,1)}$ & 0,0000 & $-0,0015$ & {$[-0,0072 ; 0,0055]$} & $-0,0012$ & 0,0033 \\
\hline
\end{tabular}


Apresentamos nas Figuras 5.9, 5.10, 5.11 e 5.12 os histogramas bootstrap dos parâmetros do modelo. Podemos notar em todas as figuras que os valores teóricos dos parâmetros estão dentro dos intervalos de confiança bootstrap de 95\%. Pelas figuras ainda é possível comparar a estimativa de cada parâmetro do modelo com sua respectiva média boostrap.

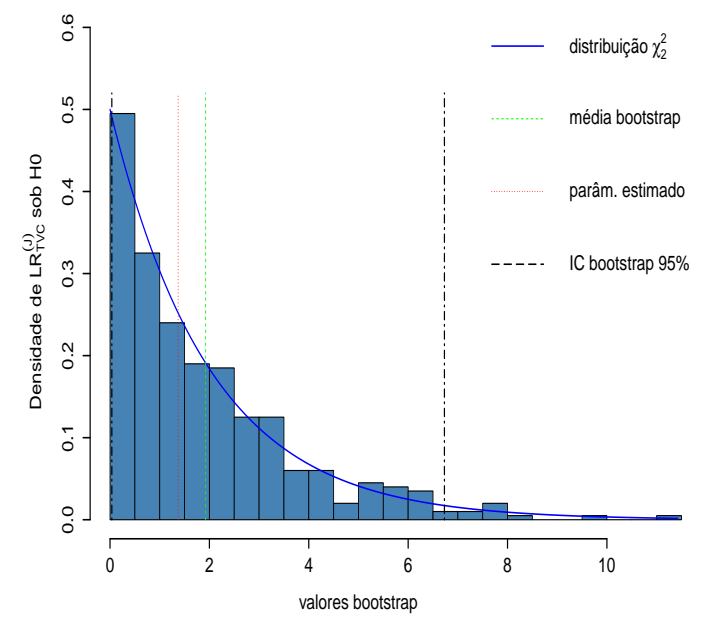

Figura 5.9: Segundo exemplo: Histograma, densidade e quantis bootstrap 2,5\% e 97,5\% para $R V_{C V T}^{(J)}$ sob H0 (vetor de cointegração constante).

(a)

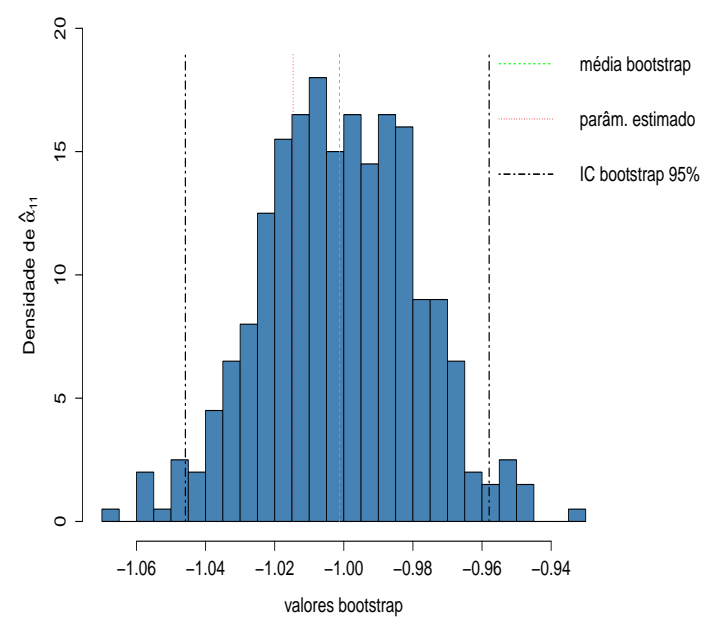

(b)

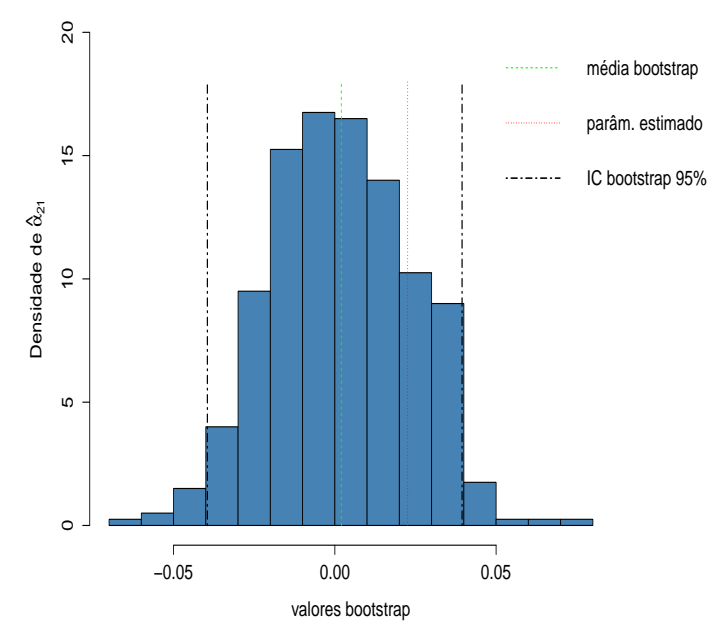

Figura 5.10: Segundo exemplo: Histograma, densidade e quantis bootstrap 2, 5\% e $97,5 \%$ sob $\mathrm{H} 0$ (vetor de cointegração constante) para as componentes $\hat{\alpha}_{11}^{*}(\mathbf{a})$ e $\hat{\alpha}_{21}^{*}(\mathbf{b})$ de $\hat{\boldsymbol{\alpha}}^{*}=\left[\hat{\alpha}_{11}^{*} \hat{\alpha}_{21}^{*}\right]^{\prime}$. 
(a)

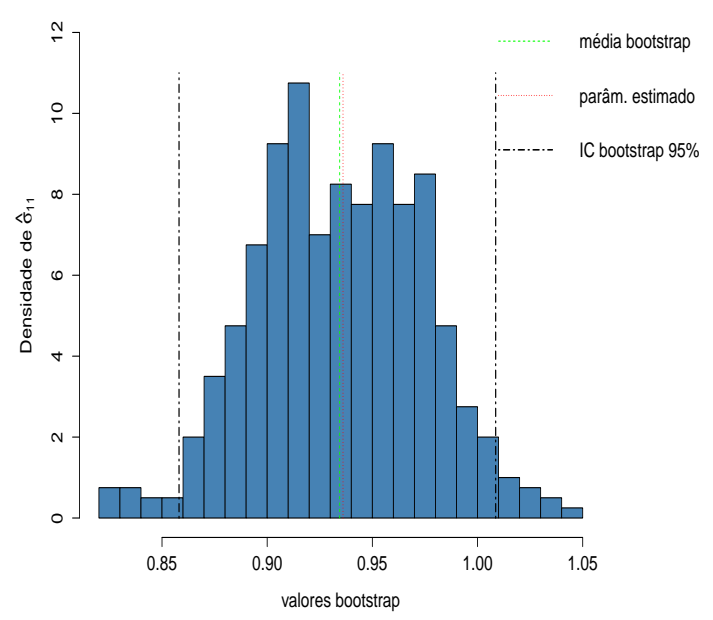

(b)

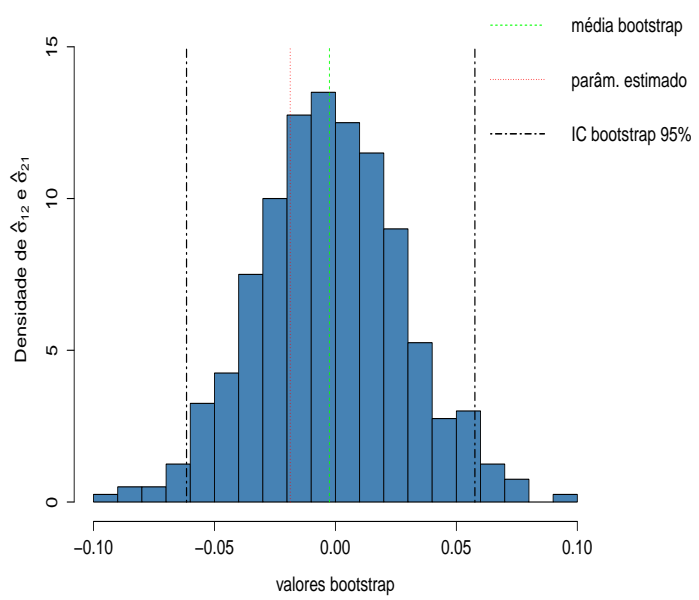

(c)

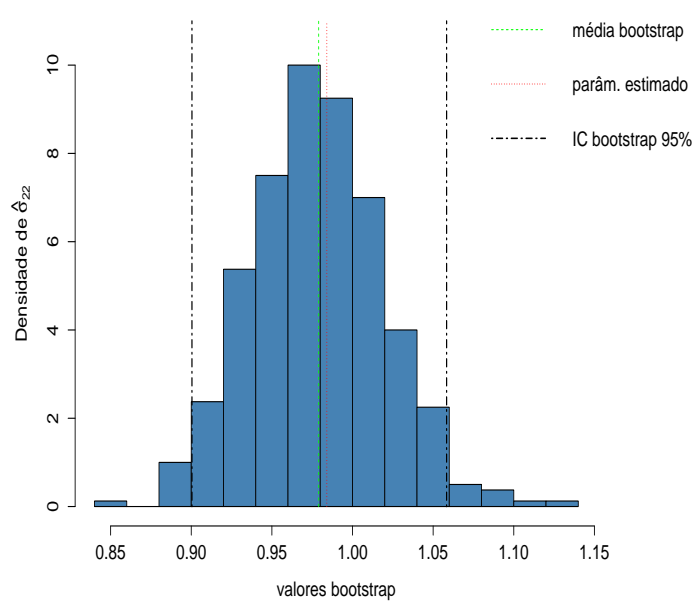

Figura 5.11: Segundo exemplo: Histograma, densidade e quantis bootstrap 2,5\% e 97,5\% sob H0 (vetor de cointegração constante) para a componente $\hat{\sigma}_{11}^{*}(\mathbf{a})$, componentes $\hat{\sigma}_{12}^{*}$ e $\hat{\sigma}_{21}^{*}$ (b) e componente $\hat{\sigma}_{22}^{*}(\mathbf{c})$ de $\hat{\boldsymbol{\Sigma}}$.

(a)

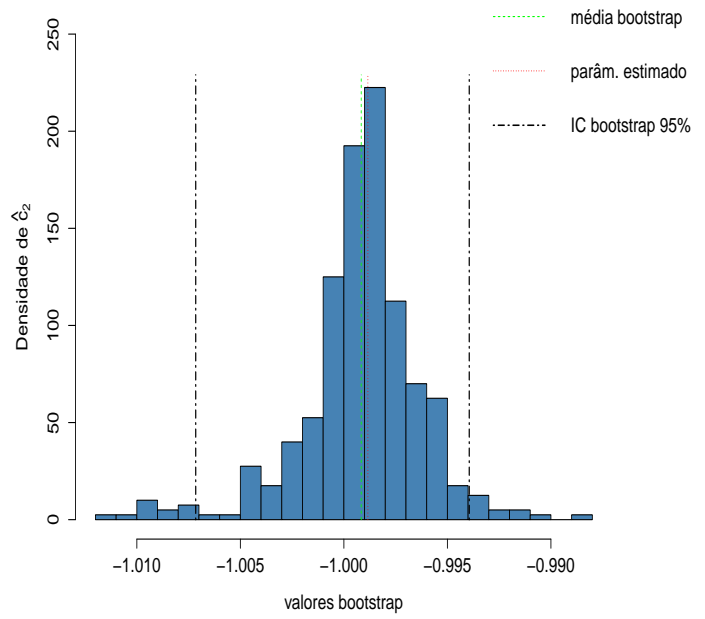

(b)

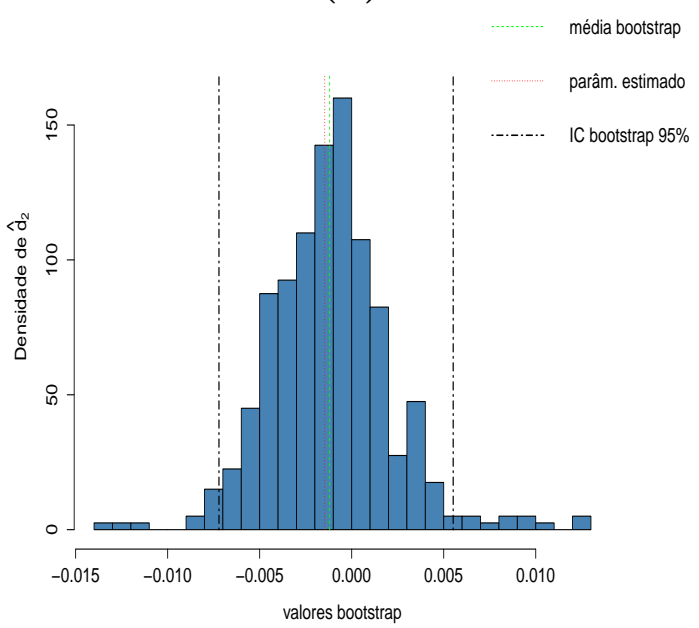

Figura 5.12: Segundo exemplo: Histograma, densidade e quantis bootstrap 2,5\% e 97,5\% sob H0 (vetor de cointegração constante) para as componentes $\hat{c}_{0,0}^{*(2,1)}$ (a) e $\hat{d}_{0,0}^{*(2,1)}$ (b) de $\hat{\boldsymbol{W}}_{J}=$

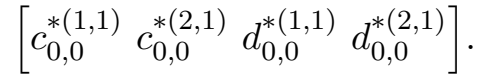


Precisamos considerar a situação mais geral, em que temos um vetor (ou matriz) de cointegração para cada instante de tempo $t$. Para este caso, precisamos de outras ordens para o modelo VEC com cointegração variando com o tempo, além da ordem $J=0$ apresentada nos exemplos anteriores. Desta forma, apresentamos a seguir alguns modelos cointegrados simulados onde necessitamos de ordens mais altas para o parâmetro $J$. Com isso, exploramos a versatilidade do modelo, permitindo estimar outras estruturas para o vetor de cointegração variando com o tempo.

\subsubsection{Terceiro exemplo: Três escalas ao longo do tempo com Haar}

Neste exemplo, permitimos ao vetor de cointegração $\boldsymbol{\beta}_{t}^{*}$ assumir três regimes ao longo do tempo, com $T=1024$. Consideramos o vetor de cointegração teórico:

$$
\boldsymbol{\beta}_{t}=\left[\begin{array}{l}
\beta_{1 t} \\
\beta_{2 t}
\end{array}\right]=\left\{\begin{array}{l}
{\left[\begin{array}{ll}
1 & -1
\end{array}\right]^{\prime}, \text { se } 1 \leq t \leq T / 4} \\
{\left[\begin{array}{ll}
1 & -2
\end{array}\right]^{\prime}, \text { se } \quad T / 4+1 \leq t \leq T / 2} \\
{\left[\begin{array}{ll}
1 & -4
\end{array}\right]^{\prime}, \text { se } \quad T / 2+1 \leq t \leq T}
\end{array}\right.
$$

Utilizando a expressão 4.1 e $J=1$, podemos escrever o vetor $\boldsymbol{\beta}_{t}^{*}$ por meio da seguinte expressão:

$$
\boldsymbol{\beta}_{t}^{*}=\phi(t) \mathbf{c}^{*}+\psi_{0,0}(t) \mathbf{d}_{0,0}^{*}+\psi_{1,0}(t) \mathbf{d}_{1,0}^{*}+\psi_{1,1}(t) \mathbf{d}_{1,1}^{*}
$$

Por 4.2 temos que $\mathbf{c}^{*}=\left[\begin{array}{lll}c_{0,0}^{*(1,1)} & c_{0,0}^{*(2,1)}\end{array}\right]^{\prime}, \mathbf{d}_{0,0}^{*}=\left[\begin{array}{ll}d_{0,0}^{*(1,1)} & d_{0,0}^{*(2,1)}\end{array}\right]^{\prime}, \mathbf{d}_{1,0}^{*}=\left[\begin{array}{ll}d_{1,0}^{*(1,1)} & d_{1,0}^{*(2,1)}\end{array}\right]^{\prime} \mathrm{e}$ $\mathbf{d}_{1,1}^{*}=\left[\begin{array}{ll}d_{1,1}^{*(1,1)} & d_{1,1}^{*(2,1)}\end{array}\right]^{\prime}$, cujos elementos são estimados por $\hat{\boldsymbol{W}}_{J}^{*}$ de 4.67 . Além disso, $\phi(t)$ e $\psi_{0,0}(t)$ são funções da base de ondaletas Haar.

Apresentamos na Figura 5.13 (a) os gráficos das séries cointegradas simuladas $Y_{1 t}$ e $Y_{2 t}$. Para estas séries, apresentamos o segundo componente dos vetores de cointegração teórico $\left(\beta_{2 t}^{*}\right)$ e estimado $\left(\hat{\beta}_{2 t}^{*}\right)$ : com $J=0$ em 5.13 (b) e com $J=1$ em 5.13 (d). Para $J=1$, obtivemos uma melhor estimativa, onde é possível notar que $\hat{\beta}_{2 t}^{*}$ está próximo de $\beta_{2 t}^{*}$, com ambos dentro das bandas de confiança de 95\%. Além disso, apresentamos as estimativas dos parâmetros do modelo, juntamente com os valores teóricos em parênteses, para $J=1$ :

$$
\begin{aligned}
& \hat{\boldsymbol{\beta}}_{t}^{*}=\left[\begin{array}{ccccccccc}
1 & \ldots & 1 & 1 & \ldots & 1 & 1 & \ldots & 1 \\
-0,9918 & \ldots & -0,9918 & -2,0051 & \ldots & -2,0051 & -4,0016 & \ldots & -4,0016 \\
(-1,0000) & & (-1,0000) & (-2,0000) & & (-2,0000) & (-4,0000) & & (-4,0000)
\end{array}\right], \\
& \hat{\boldsymbol{\alpha}}^{*}=\left[\begin{array}{c}
-0,9908 \\
(-1,0000) \\
0,0057 \\
(0,0000)
\end{array}\right] \text { e } \hat{\Sigma}_{u}^{*}=\left[\begin{array}{cc}
0,9276 & -0,0177 \\
(1,0000) & (0,0000) \\
-0,0177 & 0,9774 \\
(0,0000) & (1,0000)
\end{array}\right]
\end{aligned}
$$


Apresentamos na Figura 5.13 (c) os resíduos de cointegração teóricos $\left(\boldsymbol{\beta}_{t}^{*^{\prime}} Y_{t-1}\right)$ e estimados $\left(\hat{\boldsymbol{\beta}}_{t}^{*^{\prime}} Y_{t-1}\right)$. Verificamos que $\hat{\boldsymbol{\beta}}_{t}^{\prime *} Y_{t-1}$ correspondem a um processo estacionário (valor- $p<0,01$ para o teste DFA - Dickey-Fuller Aumentado). No entanto, verificamos que apesar de o teste apontar para a estacionariedade da série $\hat{\boldsymbol{\beta}}_{t}^{\prime *} Y_{t-1}$, com três regimes para o vetor de cointegração $\boldsymbol{\beta}_{t}$, é possível notar que exitem variâncias diferentes para cada regime, conforme pode ser obsevardo em 5.13 (c).

(a)

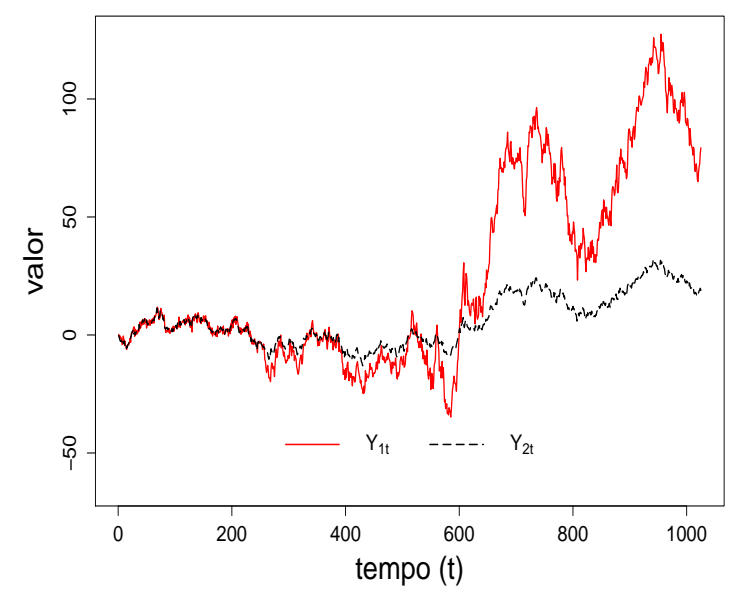

(c)

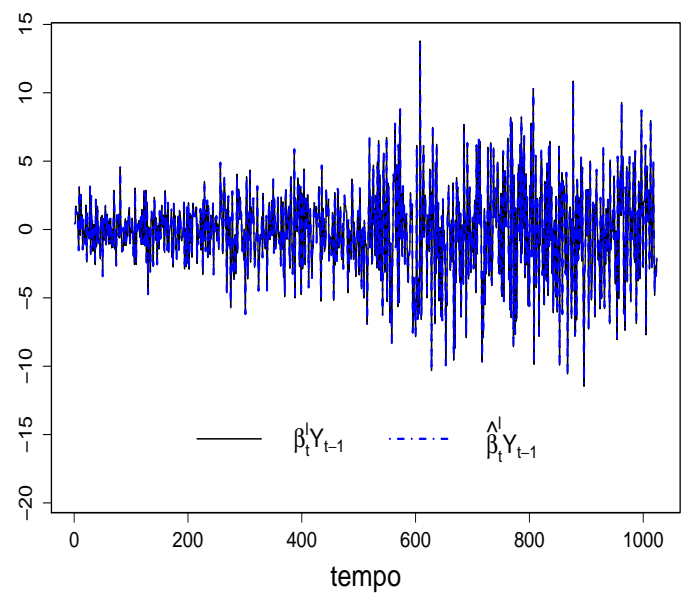

(b)

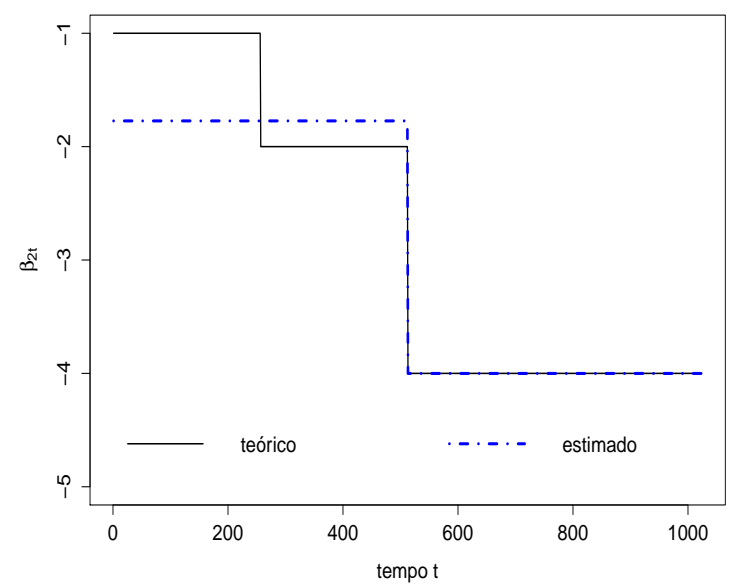

(d)

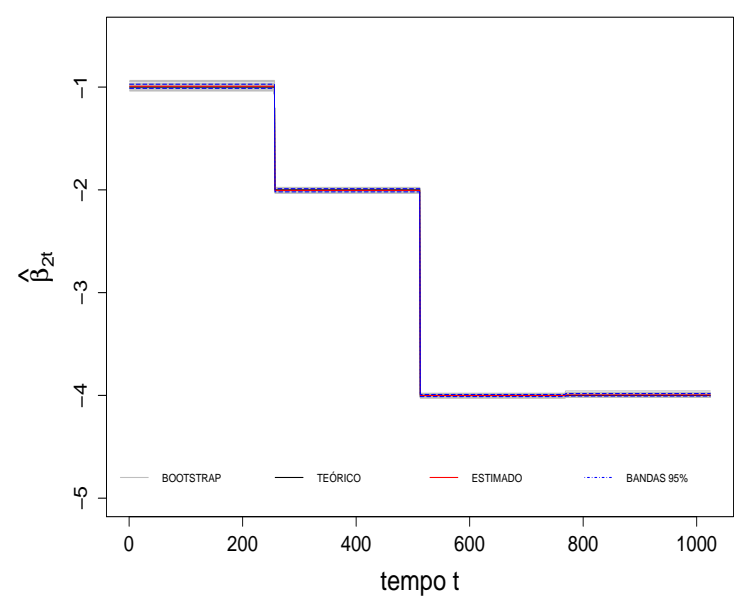

Figura 5.13: Terceiro exemplo: (a) Séries cointegradas simuladas $Y_{1 t}$ e $Y_{2 t}$, com $T=1024$, (b) componente teórico $\beta_{2 t}^{*}$ de $\boldsymbol{\beta}_{t}^{*}=\left[\beta_{1 t}^{*} \beta_{2 t}^{*}\right]^{\prime}$ e estimado $\hat{\beta}_{2 t}^{*}$ de $\hat{\boldsymbol{\beta}}_{t}^{*}=\left[\hat{\beta}_{1 t}^{*} \hat{\beta}_{2 t}^{*}\right]^{\prime}$, para $J=0$, (c) resíduos de cointegração teóricos $\left(\boldsymbol{\beta}_{t}^{\prime *} Y_{t-1}\right)$ e estimados $\left(\hat{\boldsymbol{\beta}}_{t}^{\prime *} Y_{t-1}\right)$ para $J=1$ e (d) componente teórico $\beta_{2 t}^{*}$ de $\boldsymbol{\beta}_{t}^{*}=\left[\beta_{1 t}^{*} \beta_{2 t}^{*}\right]^{\prime}$ e estimado $\hat{\beta}_{2 t}^{*}$ de $\hat{\boldsymbol{\beta}}_{t}^{*}=\left[\hat{\beta}_{1 t}^{*} \hat{\beta}_{2 t}^{*}\right]^{\prime}$, para $J=1$.

Apresentamos na Figura 5.14 os resíduos $\hat{u}_{1 t}$ e $\hat{u}_{2 t}$ do modelo. Ao aplicarmos o teste de Shapiro-Wilk individualmente a $\hat{u}_{1 t}$ e $\hat{u}_{2 t}$, verificamos que eles parecem ter uma distribuição normal (valores- $p$ iguais a 0,8669 e 0,5392 respectivamente). Conforme esperado, validamos a suposição de normalidade do modelo, visto que o modelo foi simulado sob esta condição. Tal fato é confirmado pelos QQ-plots apresentados em 5.14. Além disso, os resíduos parecem ser não-correlacionados, conforme sugerem os correlogramas apresenta- 
dos na mesma figura.
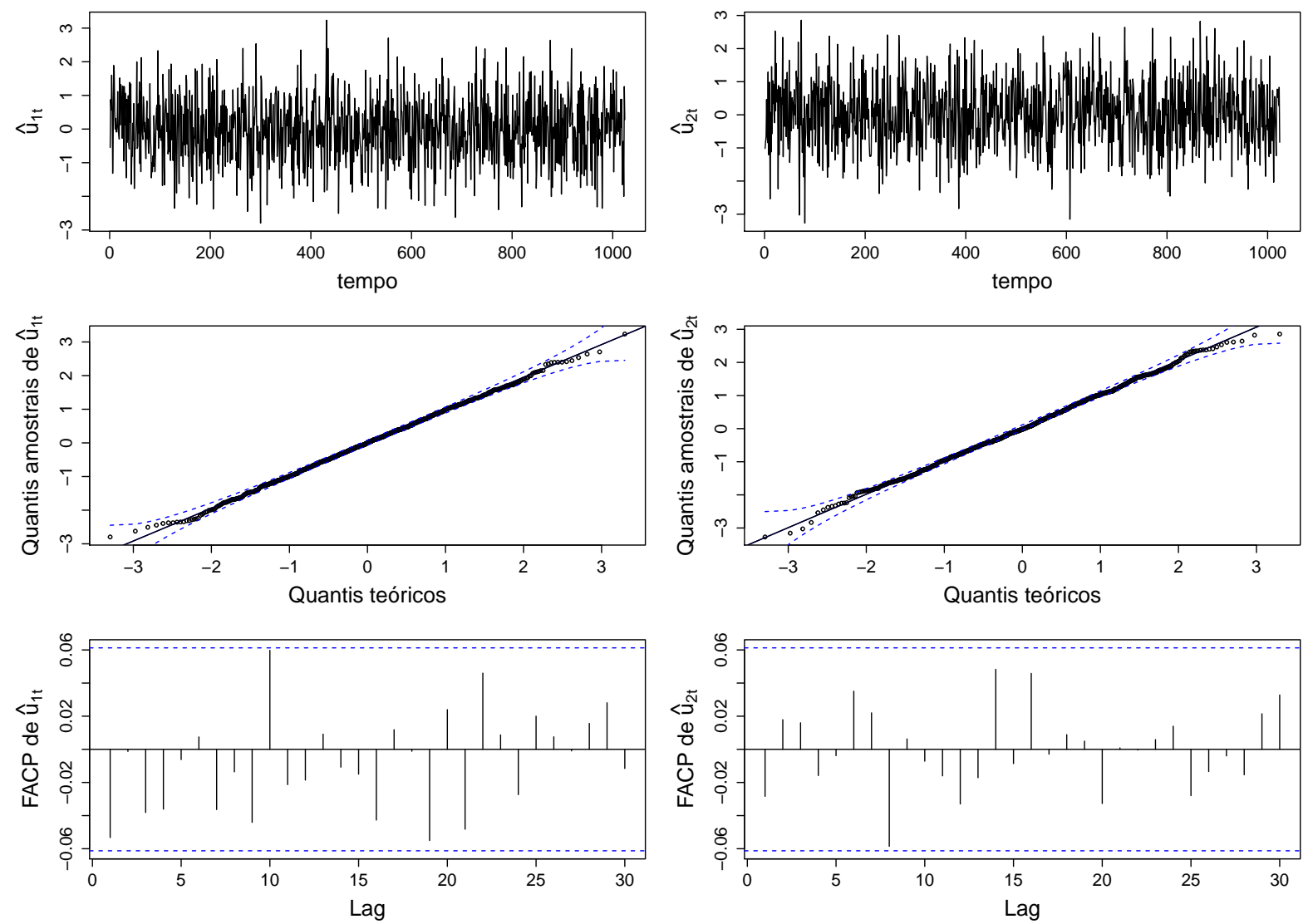

Figura 5.14: Terceiro exemplo: Resíduos estimados pelo modelo, quantis amostrais e função de autocorrelação parcial (FACP) de $\hat{u}_{t}=\left[\hat{u}_{1 t}, \hat{u}_{2 t}\right]^{\prime}$.

Por meio do valor- $p$ bootstrap apresentado na Tabela 5.10, existem evidências que nos levam a rejeitar a hipótese de cointegração constante ao longo do tempo.

Tabela 5.10: Ex.03: Estimativa e medidas bootstrap ${ }^{\star}$ sob H0 (vetor de cointegração constante).

\begin{tabular}{c|ccccc}
\hline Parâmetro & Estimativa & Intervalo de confiança & Média $^{\star}$ & Erro Padrão $^{\star}$ & valor- $p^{\star}$ \\
\hline$R V_{C V T}^{(J)}(\mathrm{H} 0)$ & 2379,43 & {$[1,2061 ; 15,4062]$} & 5,9302 & 3,6098 & 0,0000 \\
\hline
\end{tabular}

A Tabela 5.11 apresenta as estimativas dos parâmetros, bem como medidas bootstrap de interesse. Podemos notar que as estimativas e as médias bootstrap estão próximas dos valores teóricos dos parâmetros.

Apresentamos nas Figuras 5.15, 5.16, 5.17 e 5.18 os histogramas bootstrap dos parâmetros do modelo. Podemos notar em todas as figuras que os valores teóricos dos parâmetros estão dentro dos intervalos de confiança bootstrap de 95\%. Pelas figuras ainda é possível comparar a estimativa de cada parâmetro do modelo com sua respectiva média boostrap. 
Tabela 5.11: Ex.03: Estimativas e medidas bootstrap ${ }^{\star}$ sob HA (três escalas ao longo do tempo).

\begin{tabular}{c|ccccc}
\hline Parâmetro & Teórico & Estimativa & Intervalo de confiança & Média $^{\star}$ & Erro Padrão $^{\star}$ \\
\hline$R V_{C V T}^{(J)}(\mathrm{HA})$ & - & 2379,43 & {$[2272,01 ; 3235,92]$} & 2627,27 & 237,89 \\
$\alpha_{11}^{*}$ & $-1,000$ & $-0,9908$ & {$[-1,0183 ;-0,9822]$} & $-0,9999$ & 0,0095 \\
$\alpha_{21}^{*}$ & 0,0000 & 0,0057 & {$[-0,0171 ; 0,0189]$} & 0,0010 & 0,0093 \\
$\sigma_{11}^{*}$ & 1,0000 & 0,9276 & {$[0,7616 ; 1,0799]$} & 0,9275 & 0,0800 \\
$\sigma_{12}^{*}=\sigma_{21}^{*}$ & 0,0000 & $-0,0177$ & {$[-0,0582 ; 0,0501]$} & $-0,0008$ & 0,0289 \\
$\sigma_{22}^{*}$ & 1,0000 & 0,9774 & {$[0,8139 ; 1,1885]$} & 0,9795 & 0,0891 \\
$\hat{c}_{0,0}^{*(2,1)}$ & $-2,7500$ & $-2,7486$ & {$[-2,7549 ;-2,7410]$} & $-2,7484$ & 0,0034 \\
$\hat{d}_{0,0}^{*(2,1)}$ & 1,2500 & 1,2501 & {$[1,2430 ; 1,2576]$} & 1,2503 & 0,0033 \\
$\hat{d}_{1,0}^{*(2,1)}$ & 0,3535 & 0,3582 & {$[0,3489 ; 0,3658]$} & 0,3581 & 0,0043 \\
$\hat{d}_{1,1}^{*(2,1)}$ & 0,0000 & $-0,0021$ & {$[-0,0084 ; 0,0044]$} & $-0,0020$ & 0,0029 \\
\hline
\end{tabular}

(a)

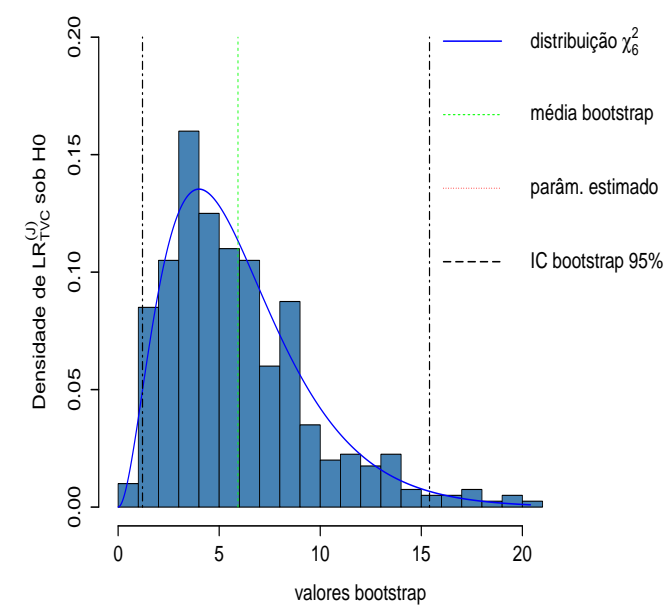

(b)

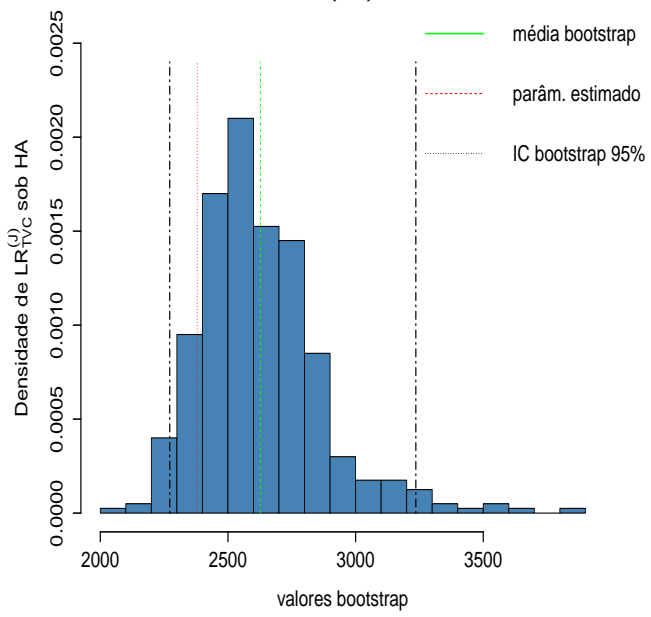

Figura 5.15: Terceiro exemplo: Histograma, densidade e quantis bootstrap 2,5\% e 97,5\% para $R V_{C V T}^{(J)}$ sob H0 (vetor de cointegração constante) (a) e sob HA (três escalas ao longo do tempo) (b).

(a)

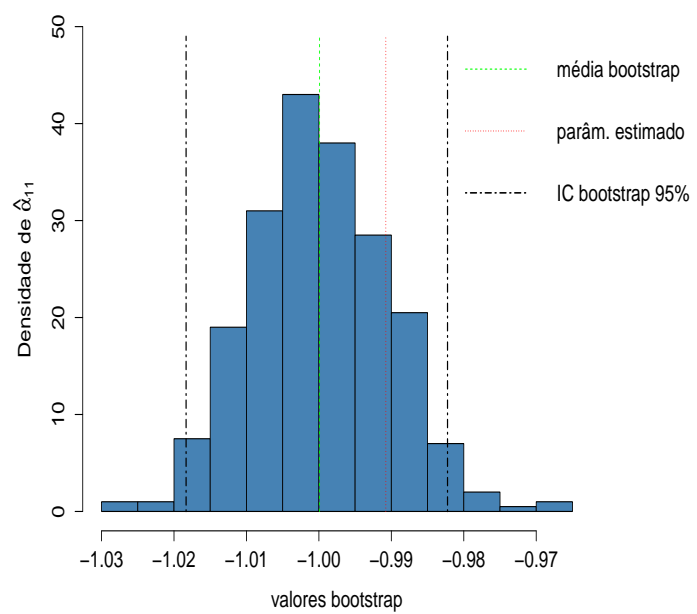

(b)

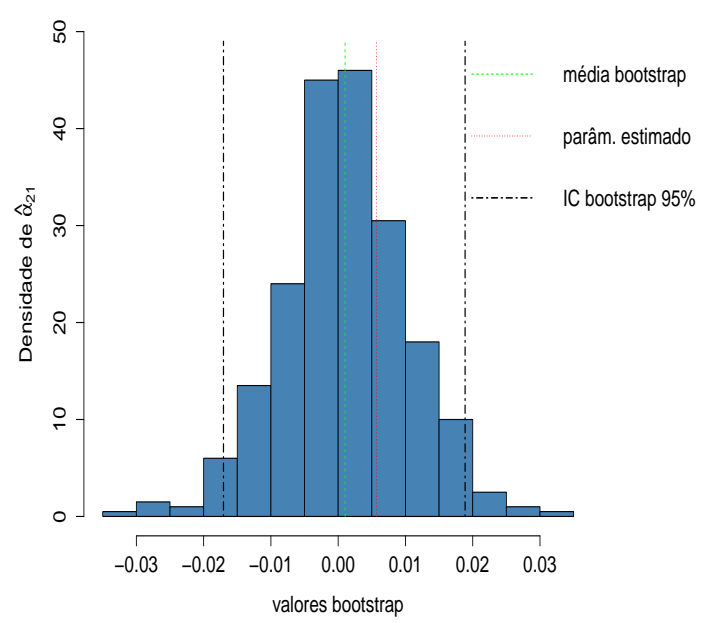

Figura 5.16: Terceiro exemplo: Histograma, densidade e quantis bootstrap 2,5\% e 97,5\% sob

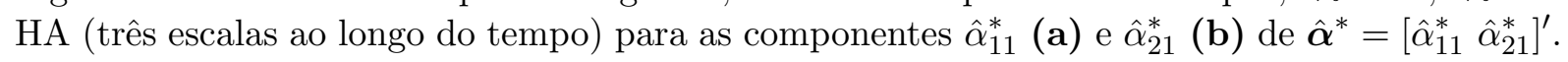


(a)

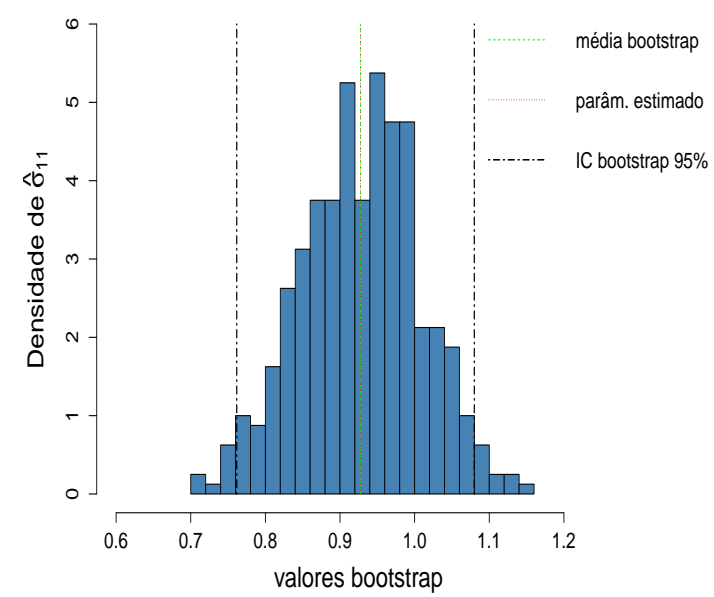

(b)

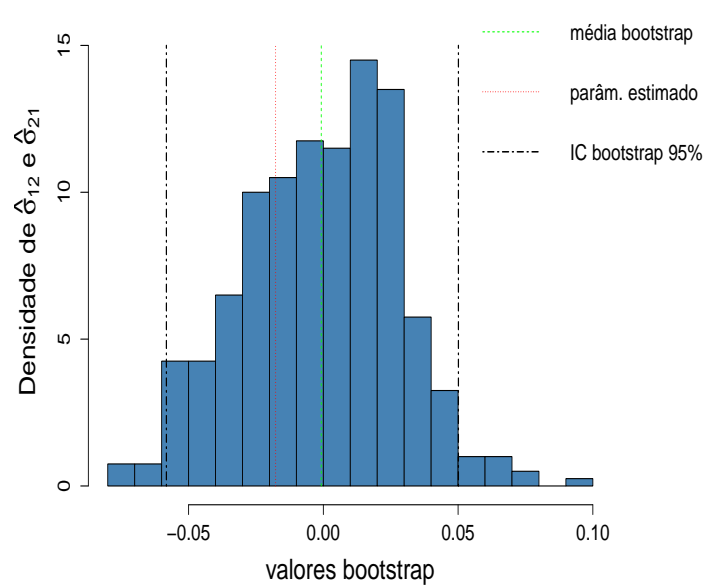

(c)

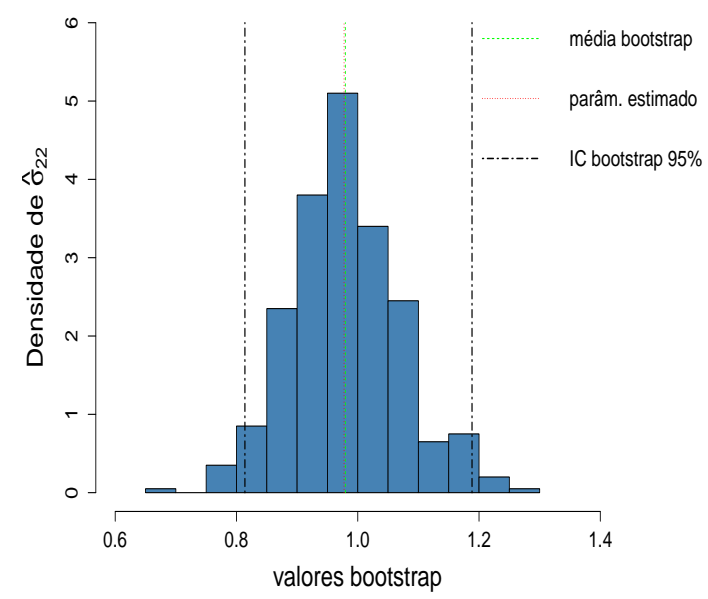

Figura 5.17: Terceiro exemplo: Histograma, densidade e quantis bootstrap 2,5\% e $97,5 \%$ sob HA (três escalas ao longo do tempo) para a componente $\hat{\sigma}_{11}^{*}$ (a), componentes $\hat{\sigma}_{12}^{*}$ e $\hat{\sigma}_{21}^{*}$ (b) e componente $\hat{\sigma}_{22}^{*}$ (c) de $\hat{\mathbf{\Sigma}}^{*}$.

(a)

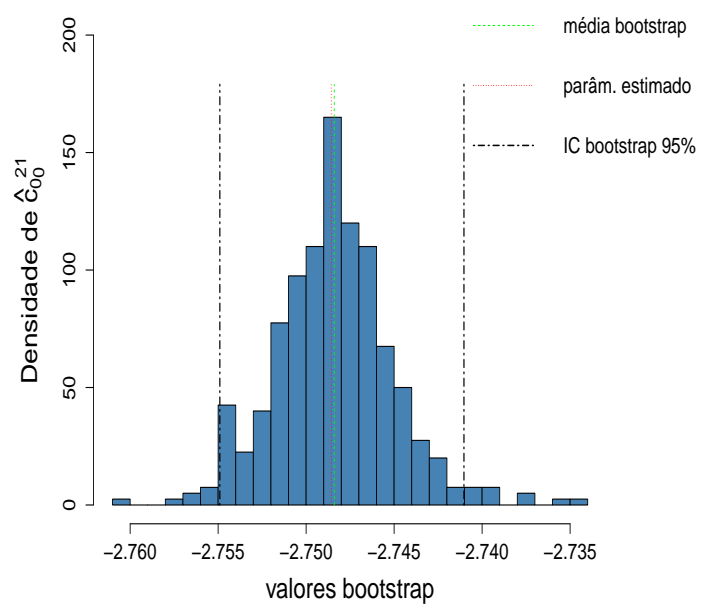

(b)

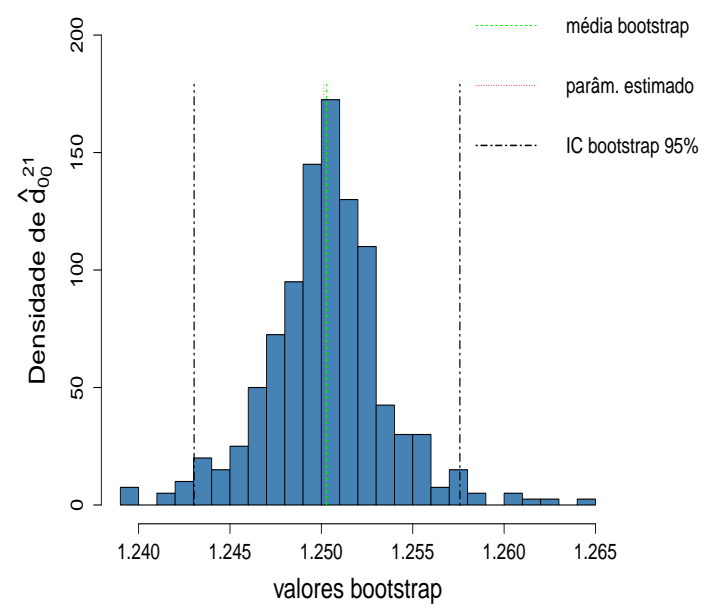

Figura 5.18: Terceiro exemplo: Histograma, densidade e quantis bootstrap 2,5\% e 97,5\% sob HA (três escalas ao longo do tempo) para as componentes $\hat{c}_{0,0}^{*(2,1)}$ (a) e $\hat{d}_{0,0}^{*(2,1)}$ (b) de $\hat{\boldsymbol{W}}_{J}=$

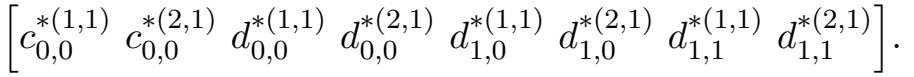


(c)

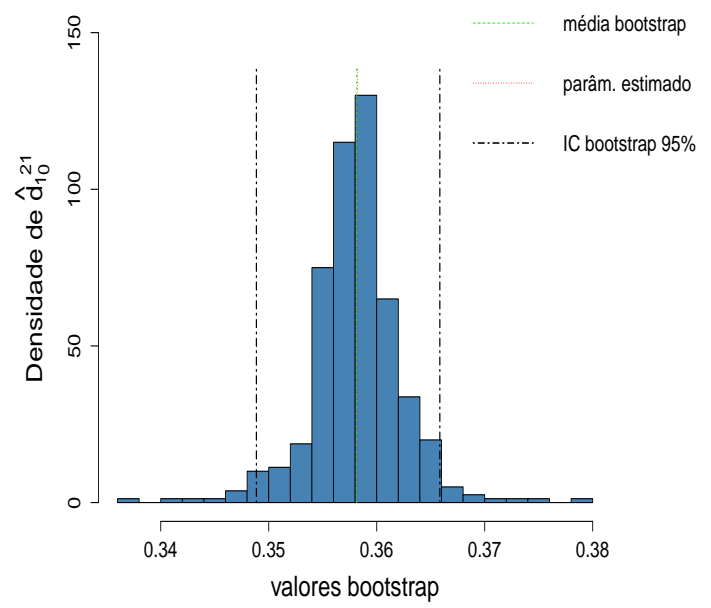

(d)

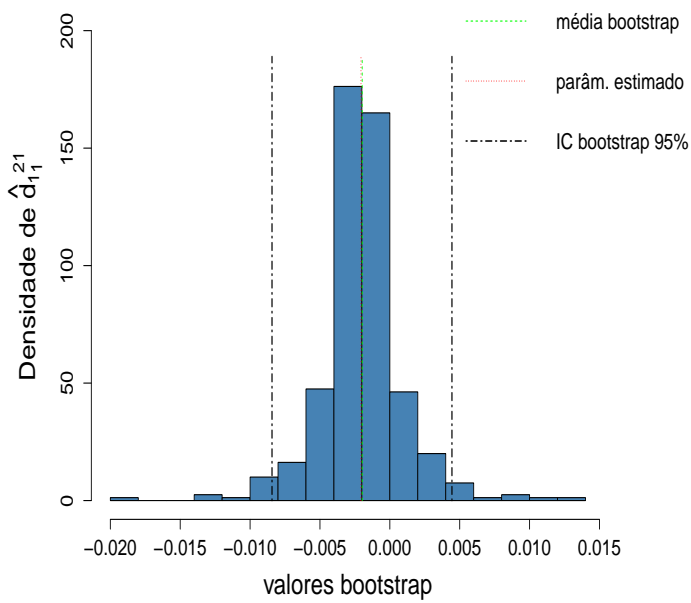

Figura 5.18: Terceiro exemplo (continuação): Histograma, densidade e quantis bootstrap 2,5\% e $97,5 \%$ sob HA (três escalas ao longo do tempo) para as componentes $\hat{d}_{1,0}^{*(2,1)}$ (c) e $\hat{d}_{1,1}^{*(2,1)}$ (d)

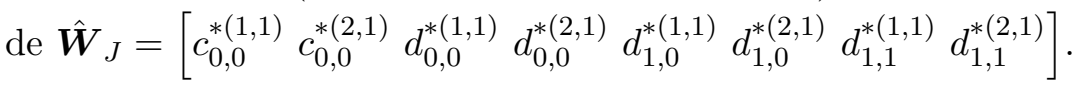

\subsubsection{Quarto exemplo: Decrescimento linear com Daubechies D2}

Nesta simulação, consideramos a componente teórica $\beta_{2 t}^{*}$ de $\boldsymbol{\beta}_{t}^{*}=\left[\beta_{1 t}^{*} \beta_{2 t}^{*}\right]^{\prime}$ descrevendo um decrescimento linear ao longo tempo, com $t=1, \ldots, T$ e $T=1024$. Para isso, consideramos o vetor de cointegração teórico:

$$
\boldsymbol{\beta}_{t}^{*}=\left[\begin{array}{l}
\beta_{1 t}^{*} \\
\beta_{2 t}^{*}
\end{array}\right]=\left[\begin{array}{c}
1 \\
-\frac{t}{T}
\end{array}\right],
$$

Utilizando a expressão 4.1 e $J=5$, podemos escrever o vetor $\boldsymbol{\beta}_{t}^{*}$ por meio da seguinte expressão:

$$
\boldsymbol{\beta}_{t}^{*}=\phi(t) \mathbf{c}^{*}+\psi_{0,0}(t) \mathbf{d}_{0,0}^{*}+\psi_{1,0}(t) \mathbf{d}_{1,0}^{*}+\psi_{1,1}(t) \mathbf{d}_{1,1}^{*}+\ldots+\psi_{5,31}(t) \mathbf{d}_{5,31}^{*}
$$

Por 4.2 temos que $\mathbf{c}^{*}=\left[\begin{array}{lll}c_{0,0}^{*(1,1)} & c_{0,0}^{*(2,1)}\end{array}\right]^{\prime}$ e $\mathbf{d}_{J, 2^{J}-1}^{*}=\left[\begin{array}{ll}\hat{d}_{J, 2^{J}-1}^{*(1,1)} & \hat{d}_{J, 2^{J}-1}^{*(2,1)}\end{array}\right]^{\prime}$, cujos elementos são estimados por $\hat{\boldsymbol{W}}_{J}^{*}$ de 4.68 . Além disso, $\phi(t)$ e $\psi_{0,0}(t)$ são funções da base de ondaletas Daubechies D2.

Apresentamos na Figura 5.19 (a) os gráficos das séries cointegradas simuladas $Y_{1 t}$ e $Y_{2 t}$. Para estas séries, apresentamos em 5.19 (b) o segundo componente dos vetores de cointegração teórico $\left(\beta_{2 t}^{*}\right)$ e estimado $\left(\hat{\beta}_{2 t}^{*}\right)$. Pela figura, é possível notar que $\hat{\beta}_{2 t}^{*}$ está próximo de $\beta_{2 t}^{*}$, sendo que ambos estão dentro das bandas de confiança de $95 \%$. Além disso, apresentamos as estimativas dos parâmetros do modelo, juntamente com os valores teóricos em parênteses: 


$$
\hat{\boldsymbol{\alpha}}^{*}=\left[\begin{array}{c}
-1,0181 \\
(-1,0000) \\
-0.0008 \\
(0,0000)
\end{array}\right] \text { e } \hat{\boldsymbol{\Sigma}}_{u}^{*}=\left[\begin{array}{cc}
0,8551 & -0,0212 \\
(1,0000) & (0,0000) \\
-0,0212 & 0,8655 \\
(0,0000) & (1,0000)
\end{array}\right]
$$

Apresentamos na Figura 5.19 (c) os resíduos de cointegração teóricos $\left(\boldsymbol{\beta}_{t}^{*^{\prime}} Y_{t-1}\right)$ e estimados $\left(\hat{\boldsymbol{\beta}}_{t}^{*^{\prime}} Y_{t-1}\right)$. Verificamos que $\hat{\boldsymbol{\beta}}_{t}^{*} Y_{t-1}$ correspondem a um processo estacionário (valor$p<0,01$ para o teste DFA - Dickey-Fuller Aumentado).

(a)

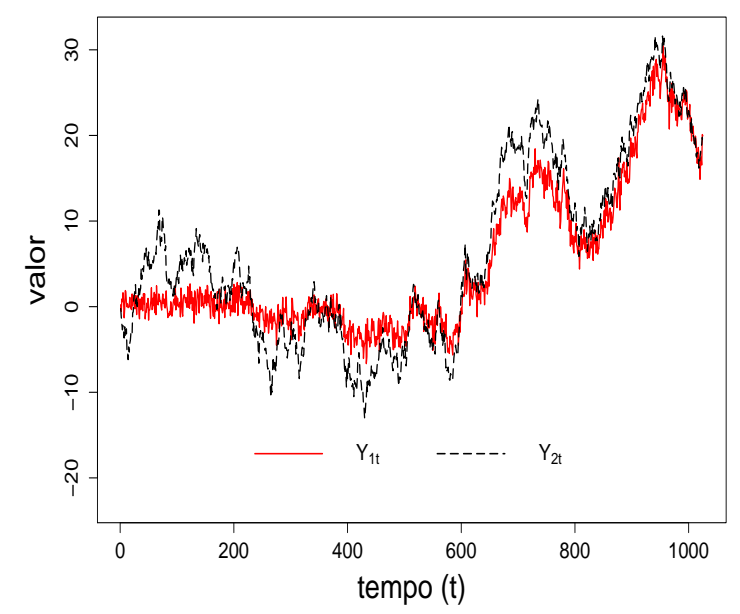

(b)

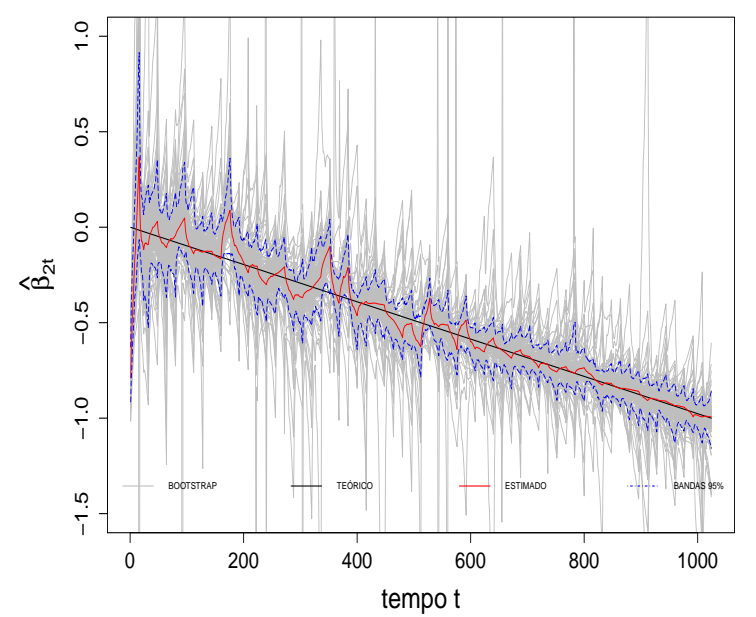

(c)

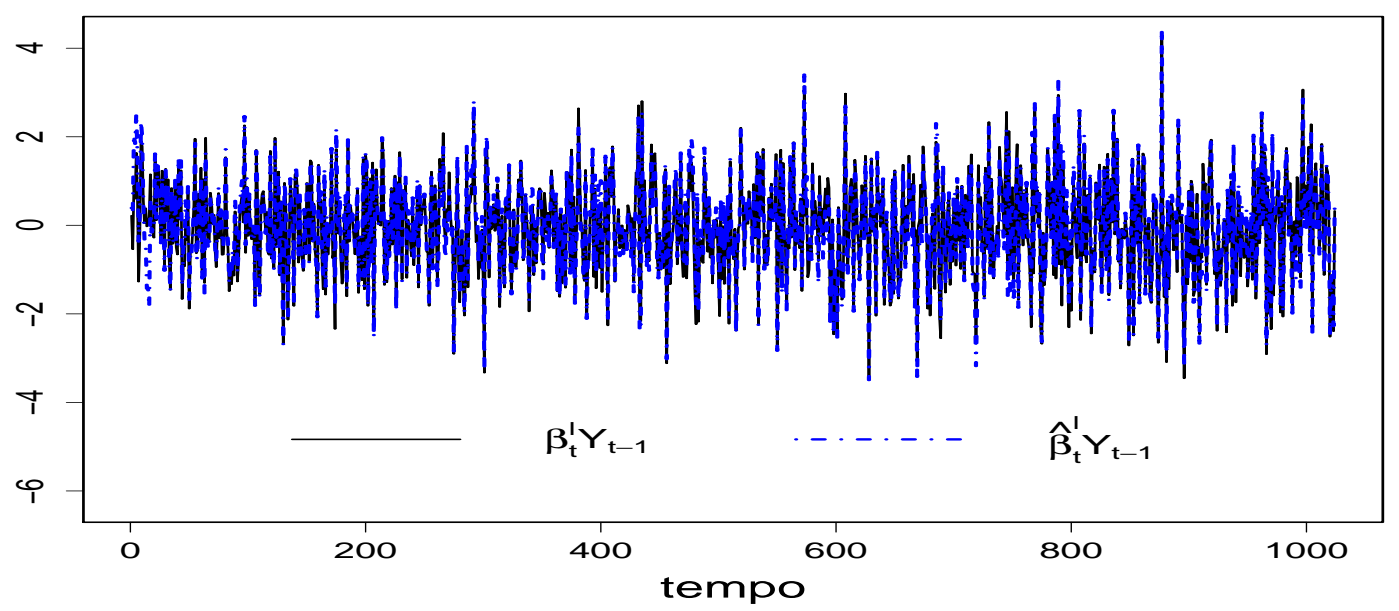

Figura 5.19: Quarto exemplo: (a) Séries cointegradas simuladas $Y_{1 t}$ e $Y_{2 t}$, com $T=1024$, (b)

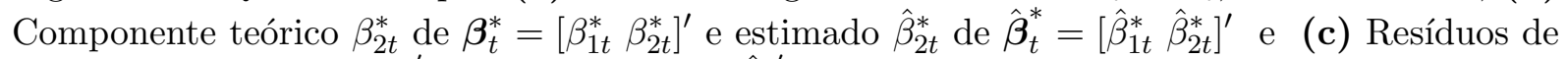
cointegração teóricos $\left(\beta_{t}^{*^{\prime}} Y_{t-1}\right)$ e estimados $\left(\hat{\beta}_{t}^{*^{\prime}} Y_{t-1}\right)$.

Apresentamos na Figura 5.20 os resíduos $\hat{u}_{1 t}$ e $\hat{u}_{2 t}$ do modelo. Ao aplicarmos o teste de Shapiro-Wilk individualmente a $\hat{u}_{1 t}$ e $\hat{u}_{2 t}$, verificamos que eles parecem ter uma distribuição normal (valores-p iguais a 0,4456 e 0,5232 respectivamente). Conforme esperado, validamos a suposição de normalidade do modelo, visto que o modelo foi simulado sob esta condição. Tal fato é confirmado pelos QQ-plots apresentados em 5.20. Além disso, os 
resíduos parecem ser não-correlacionados, conforme sugerem os correlogramas apresentados na mesma figura.
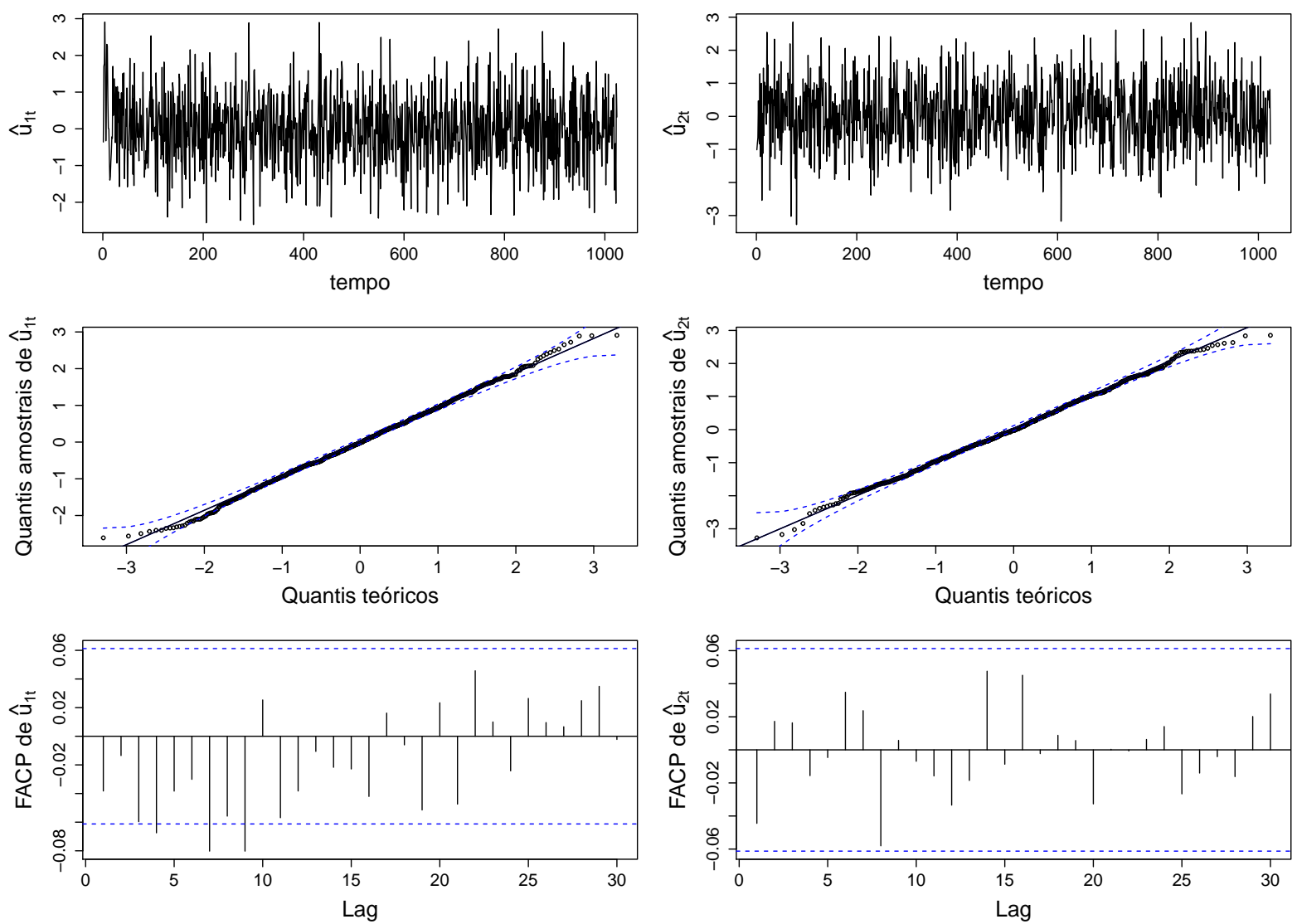

Figura 5.20: Quarto exemplo: Resíduos estimados pelo modelo, quantis amostrais e função de autocorrelação parcial (FACP) de $\hat{u}_{t}=\left[\hat{u}_{1 t}, \hat{u}_{2 t}\right]^{\prime}$.

Por meio do valor- $p$ bootstrap apresentado na Tabela 5.12, existem evidências que nos levam a rejeitar a hipótese de cointegração constante ao longo do tempo.

Tabela 5.12: Ex.04: Estimativa e medidas bootstrap ${ }^{\star}$ sob H0 (vetor de cointegração constante).

\begin{tabular}{c|ccccc}
\hline Parâmetro & Estimativa & Intervalo de confiança $^{\star}$ & Média $^{*}$ & Erro Padrão $^{*}$ & valor- $p^{*}$ \\
\hline$R V_{C V T}^{(J)}(\mathrm{H} 0)$ & 855,37 & {$[91,7051 ; 161,6428]$} & 123,7970 & 17,3768 & 0,0000 \\
\hline
\end{tabular}

Apresentamos nas Figuras 5.21, 5.22 e 5.23 os histogramas bootstrap dos parâmetros do modelo. Podemos notar em todas as figuras que os valores teóricos dos parâmetros estão dentro dos intervalos de confiança bootstrap de 95\%. Pelas figuras ainda é possível comparar a estimativa de cada parâmetro do modelo com sua respectiva média boostrap. Apesar de estarem dentro dos intervalos de confiança bootstrap, percebemos um pequeno desvio entre a estimativa e a média bootstrap para o parâmetro $R V_{C V T}^{(J)}$ sob HA. 
(a)

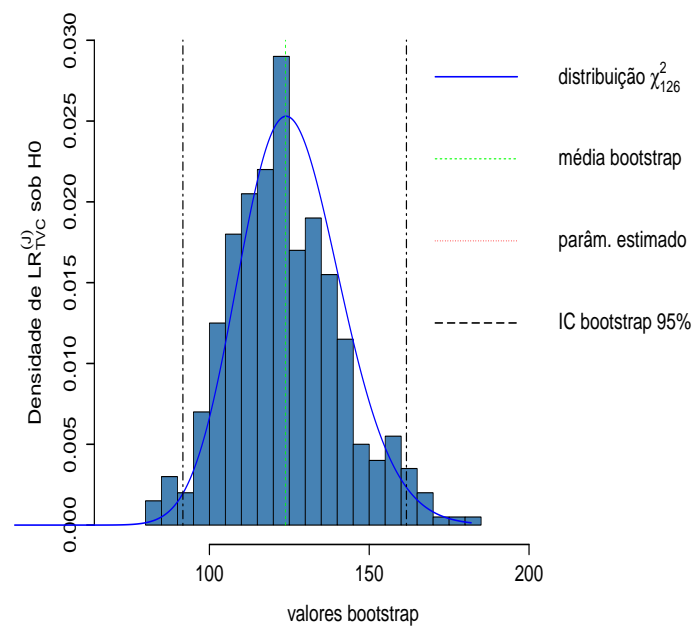

(b)

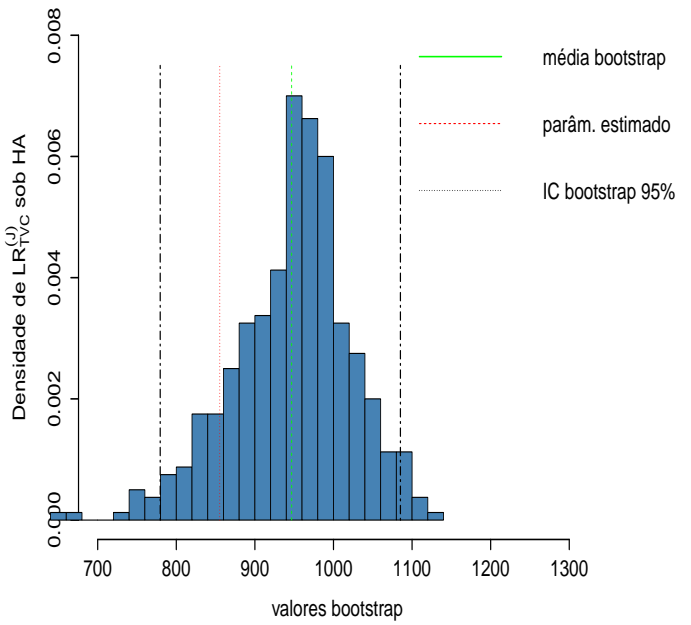

Figura 5.21: Quarto exemplo: Histograma, densidade e quantis bootstrap 2,5\% e 97,5\% para $R V_{C V T}^{(J)}$ sob H0 (vetor de cointegração constante) (a) e sob HA (decrescimento linear) (b).

(a)

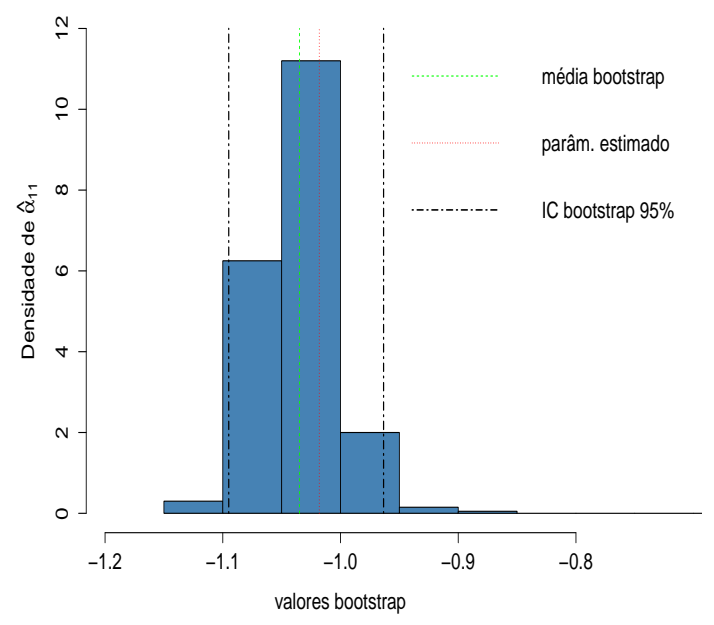

(b)

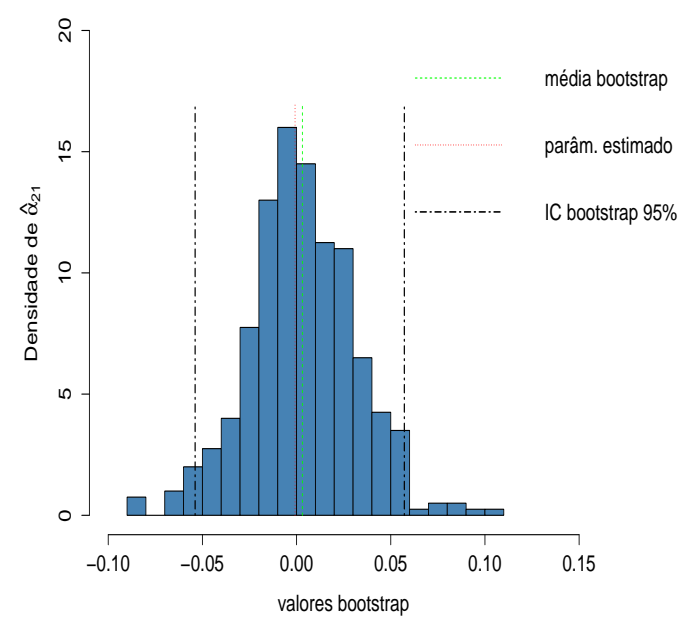

Figura 5.22: Quarto exemplo: Histograma, densidade e quantis bootstrap 2, 5\% e 97, 5\% sob HA (decrescimento linear) para as componentes $\hat{\alpha}_{11}^{*}(\mathbf{a})$ e $\hat{\alpha}_{21}^{*}$ (b) de $\hat{\boldsymbol{\alpha}}^{*}=\left[\begin{array}{ll}\hat{\alpha}_{11}^{*} & \hat{\alpha}_{21}^{*}\end{array}\right]^{\prime}$. 
(a)

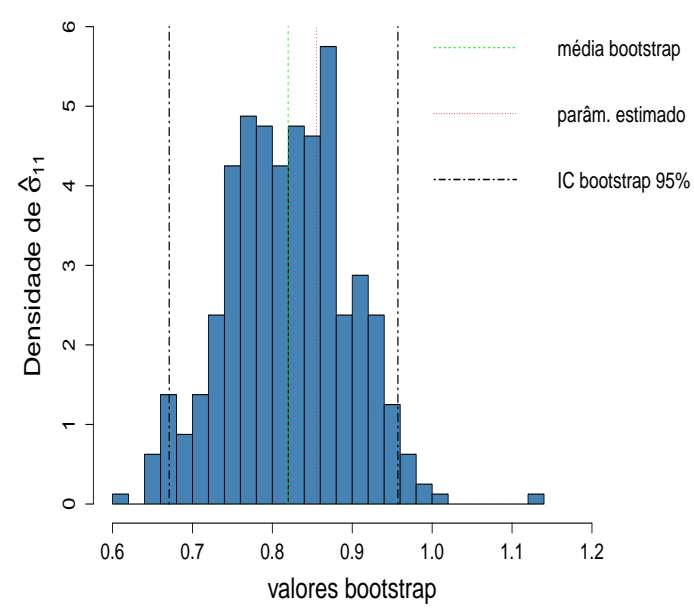

(b)

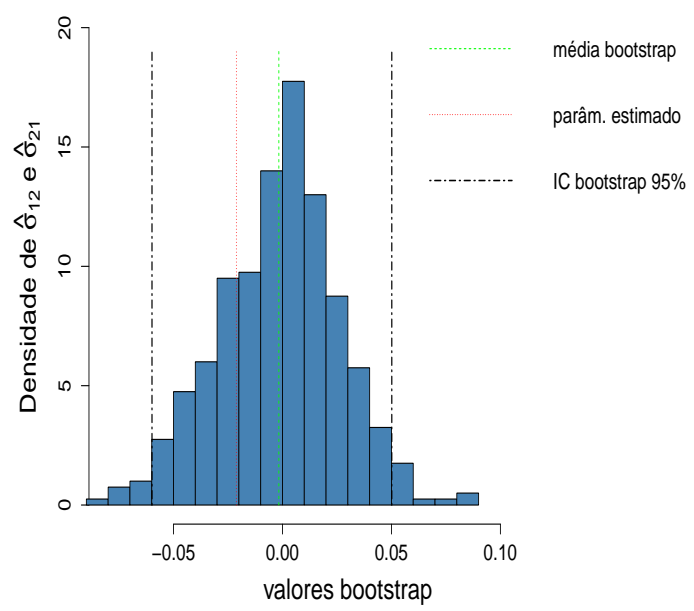

(c)

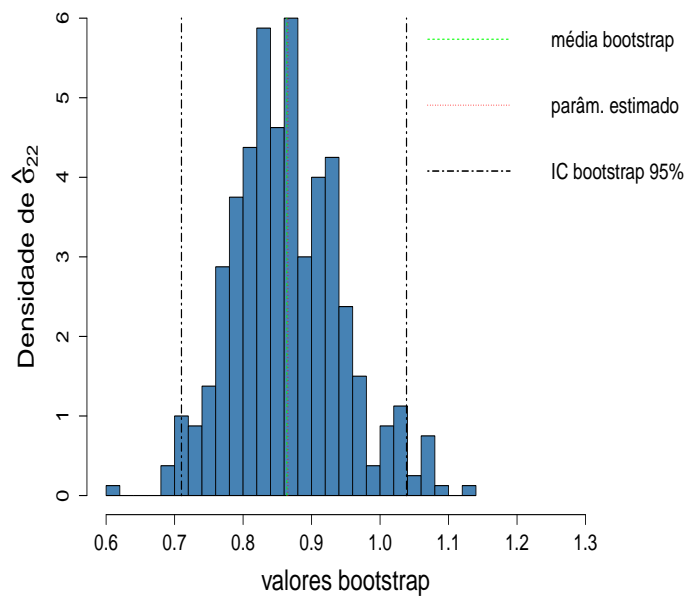

Figura 5.23: Quarto exemplo: Histograma, densidade e quantis bootstrap 2, 5\% e 97, 5\% sob HA (decrescimento linear) para a componente $\hat{\sigma}_{11}^{*}(\mathbf{a})$, componentes $\hat{\sigma}_{12}^{*}$ e $\hat{\sigma}_{21}^{*}(\mathbf{b})$ e componente $\hat{\sigma}_{22}^{*}$ (c) de $\hat{\boldsymbol{\Sigma}}^{*}$.

Apresentamos na Tabela 5.13 estimativas dos parâmetros considerados no modelo, bem como medidas bootstrap de interesse. A Tabela B.1 da Seção B.2 anexa apresenta os demais parâmetros estimados. Podemos notar que a maioria das estimativas e as médias bootstrap estão próximas dos valores teóricos dos parâmetros, que por sua vez estão dentro dos intervalos de confiança bootstrap. As excessões são os parâmetros $\sigma_{11}^{*}, d_{31}^{*(2,2)}$ e $d_{32}^{*(2,1)}$.

Tabela 5.13: Ex.04: Estimativas e medidas bootstrap* sob HA (decrescimento linear)

\begin{tabular}{c|ccccc}
\hline Parâmetro & Teórico & Estimativa & Intervalo de confiança & Média* $^{*}$ & Erro Padrão* \\
\hline$R V_{C V T}^{(J)}(\mathrm{HA})$ & - & 855,37 & {$[779,53 ; 1085,17]$} & 946,80 & 75,45 \\
\hline$\alpha_{11}^{*}$ & $-1,0000$ & $-1,0181$ & {$[-1,0949 ;-0,9634]$} & $-1,0349$ & 0,0592 \\
$\alpha_{21}^{*}$ & 0,0000 & $-0,0008$ & {$[-0,0539 ; 0,0572]$} & 0,0031 & 0,0285 \\
\hline$\sigma_{11}^{*}$ & 1,0000 & 0,8551 & {$[0,6709 ; 0,9571]$} & 0,8197 & 0,0750 \\
$\sigma_{12}^{*}=\sigma_{21}^{*}$ & 0,0000 & $-0,0212$ & {$[-0,0599 ; 0,0502]$} & $-0,0017$ & 0,0275 \\
\hline
\end{tabular}

(Continua na página seguinte) 
Tabela 5.13 - Continuação da página anterior

\begin{tabular}{|c|c|c|c|c|c|}
\hline Parâmetro & Teórico & Estimativa & Intervalo de confiança* & Média* & Erro Padrão* \\
\hline$\sigma_{22}^{*}$ & 1,0000 & 0,8655 & {$[0,7100 ; 1,0386$} & 0,8639 & 0,0802 \\
\hline$c_{00}^{*(1,1)}$ & 1,0000 & 1,0000 & {$[1,0000 ; 1,0000$} & 1,0000 & 0,0000 \\
\hline$c_{00}^{*(2,1)}$ & $-0,5005$ & $-0,4954$ & {$[-0,5144 ;-0,4788]$} & $-0,4950$ & 0,0090 \\
\hline$d_{00}^{*(1,1)}$ & 0,0000 & 0,0000 & {$[0,0000 ; 0,0000$} & 0,0000 & 0,0000 \\
\hline$d_{00}^{*(2,1)}$ & 0,1678 & 0,1717 & {$[0,1542 ; 0,1891$} & 0,1713 & 0,0081 \\
\hline$d_{10}^{*(1,1)}$ & 0,0000 & 0,0000 & {$[0,0000 ; 0,0000$} & 0,0000 & 0,0000 \\
\hline$d_{10}^{*(2,1)}$ & 0,1618 & 0,1701 & {$[0,1529 ; 0,1897$} & 0,1700 & 0,0095 \\
\hline$d_{11}^{*(1,1)}$ & 0,0000 & 0,0000 & {$[0,0000 ; 0,0000$} & 0,0000 & 0,0000 \\
\hline$d_{11}^{*(2,1)}$ & $-0,0419$ & $-0,0397$ & {$[-0,0559 ;-0,0223]$} & $-0,0398$ & 0,0076 \\
\hline$d_{20}^{*(1,1)}$ & 0,0000 & 0,0000 & {$[0,0000 ; 0,0000$} & 0,0000 & 0,0000 \\
\hline$d_{20}^{*(2,1)}$ & 0,1156 & 0,1197 & {$[0,1009 ; 0,1418$} & 0,1200 & 0,0141 \\
\hline$d_{21}^{*(1,1)}$ & 0,0000 & 0,0000 & {$[0,0000 ; 0,0000$} & 0,0000 & 0,0000 \\
\hline$d_{21}^{*(2,1)}$ & $-0,0002$ & 0,0138 & {$[-0,0057 ; 0,0317]$} & 0,0136 & 0,0085 \\
\hline$d_{22}^{*(1,1)}$ & 0,0000 & 0,0000 & {$[0,0000 ; 0,0000$} & 0,0000 & 0,0000 \\
\hline$d_{22}^{*(2,1)}$ & 0,0000 & 0,0018 & {$[-0,0193 ; 0,0120$} & 0,0010 & 0,0074 \\
\hline$d_{23}^{*(1,1)}$ & 0,0000 & 0,0000 & {$[0,0000 ; 0,0000]$} & 0,0000 & 0,0000 \\
\hline$d_{23}^{*(2,1)}$ & $-0,0282$ & $-0,0222$ & {$[-0,0390 ;-0,0080]$} & $-0,0223$ & 0,0070 \\
\hline$d_{30}^{*(1,1)}$ & 0,0000 & 0,0000 & {$[0,0000 ; 0,0000$} & 0,0000 & 0,0000 \\
\hline$d_{30}^{*(2,1)}$ & 0,0839 & 0,0797 & {$[0,0608 ; 0,1021$} & 0,0804 & 0,0113 \\
\hline$d_{31}^{*(1,1)}$ & 0,0000 & 0,0000 & {$[0,0000 ; 0,0000$} & 0,0000 & 0,0000 \\
\hline$d_{31}^{*(2,1)}$ & $-0,0004$ & 0,0170 & {$[0,0020 ; 0,0343$} & 0,0173 & 0,0086 \\
\hline$d_{32}^{*(1,1)}$ & 0,0000 & 0,0000 & {$[0,0000 ; 0,0000$} & 0,0000 & 0,0000 \\
\hline$d_{32}^{*(2,1)}$ & 0,0000 & $-0,0166$ & {$[-0,0371 ;-0,0012]$} & $-0,0174$ & 0,0085 \\
\hline$d_{33}^{*(1,1)}$ & 0,0000 & 0,0000 & {$[0,0000 ; 0,0000]$} & 0,0000 & 0,0000 \\
\hline$d_{33}^{*(2,1)}$ & 0,0000 & 0,0045 & {$[-0,0153 ; 0,0209$} & 0,0039 & 0,0096 \\
\hline$d_{34}^{*(1,1)}$ & 0,0000 & 0,0000 & {$[0,0000 ; 0,0000$} & 0,0000 & 0,0000 \\
\hline$d_{34}^{*(2,1)}$ & 0,0000 & 0,0059 & {$[-0,0119 ; 0,0193]$} & 0,0056 & 0,0078 \\
\hline$d_{35}^{*(1,1)}$ & 0,0000 & 0,0000 & {$[0,0000 ; 0,0000$} & 0,0000 & 0,0000 \\
\hline$d_{35}^{*(2,1)}$ & 0,0000 & $-0,0002$ & {$[-0,0130 ; 0,0153$} & $-0,0003$ & 0,0056 \\
\hline$d_{36}^{*(1,1)}$ & 0,0000 & 0,0000 & {$[0,0000 ; 0,0000$} & 0,0000 & 0,0000 \\
\hline$d_{36}^{*(2,1)}$ & 0,0000 & 0,0017 & {$[-0,0091 ; 0,0209$} & 0,0019 & 0,0063 \\
\hline$d_{37}^{*(1,1)}$ & 0,0000 & 0,0000 & {$[0,0000 ; 0,0000$} & 0,0000 & 0,0000 \\
\hline$d_{37}^{*(2,1)}$ & $-0,0174$ & $-0,0132$ & {$[-0,0306 ;-0,0006]$} & $-0,0136$ & 0,0071 \\
\hline$\vdots$ & $\vdots$ & $\vdots$ & $\vdots$ & $\vdots$ & $\vdots$ \\
\hline$d_{531}^{(1,1)}$ & 0,0000 & 0,0000 & {$[0,0000 ; 0,0000$} & 0,0000 & 0,0000 \\
\hline$d_{531}^{(2,1)}$ & 0,0000 & $-0,0012$ & {$[-0,0147 ; 0,0110$} & $-0,0014$ & 0,0082 \\
\hline
\end{tabular}




\subsubsection{Quinto exemplo: Transição suave entre duas escalas con- siderando limiarização e ondaleta Haar}

Consideramos novamente dois regimes ao longo do tempo para a componente $\beta_{2 t}$ de $\boldsymbol{\beta}_{t}=\left[\beta_{1 t} \beta_{2 t}\right]^{\prime}$, com $t=1, \ldots, T$ e $T=1024$. Na simulação, consideramos $\beta_{2 t}$ semelhante ao utilizado no Primeiro exemplo. No entanto, a transição entre os dois regimes ocorre de maneira suave. Para isso, consideramos o vetor de cointegração teórico:

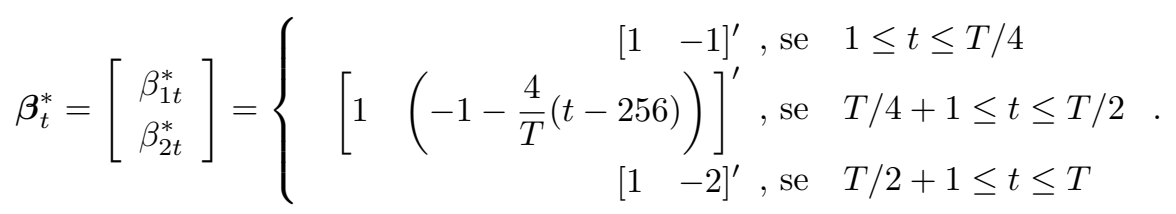

Utilizando a expressão 4.1 e $J=3$, podemos escrever o vetor $\boldsymbol{\beta}_{t}^{*}$ por meio da seguinte expressão:

$$
\boldsymbol{\beta}_{t}^{*}=\phi(t) \mathbf{c}^{*}+\psi_{0,0}(t) \mathbf{d}_{0,0}^{*}+\psi_{1,0}(t) \mathbf{d}_{1,0}^{*}+\psi_{1,1}(t) \mathbf{d}_{1,1}^{*}+\ldots+\psi_{3,7}(t) \mathbf{d}_{3,7}^{*} .
$$

Por 4.2 temos que $\mathbf{c}^{*}=\left[\begin{array}{lll}c_{0,0}^{*(1,1)} & c_{0,0}^{*(2,1)}\end{array}\right]^{\prime}$ e $\mathbf{d}_{J, 2^{J}-1}^{*}=\left[\begin{array}{ll}\hat{d}_{J, 2^{J}-1}^{*(1,1)} & \hat{d}_{J, 2^{J}-1}^{*(2,1)}\end{array}\right]^{\prime}$, cujos elementos são estimados por $\hat{\boldsymbol{W}}_{J}^{*}$ de 4.68. Além disso, $\phi(t)$ e $\psi_{0,0}(t)$ são funções da base de ondaletas Haar.

Apresentamos na Figura 5.24 (a) os gráficos das séries cointegradas simuladas $Y_{1 t}$ e $Y_{2 t}$. Para estas séries, apresentamos em 5.24 (b) o segundo componente dos vetores de cointegração teórico $\left(\beta_{2 t}^{*}\right)$ e estimado $\left(\hat{\beta}_{2 t}^{*}\right)$. Pela figura, é possível notar que $\hat{\beta}_{2 t}^{*}$ está próximo de $\beta_{2 t}^{*}$, sendo que ambos estão dentro das bandas de confiança de $95 \%$ entre $1 \leq t \leq T / 4$ e $T / 2+1 \leq t \leq T$. Percebemos que os valores estimados (e bandas de confiança) se desviam dos valores teóricos entre no intervalo $T / 4+1 \leq t \leq T / 2$. Em 5.24 (c), apresentamos os resíduos de cointegração teóricos $\left(\boldsymbol{\beta}_{t}^{*^{\prime}} Y_{t-1}\right)$ e estimados $\left(\hat{\boldsymbol{\beta}}_{t}^{*^{\prime}} Y_{t-1}\right)$. Verificamos que $\hat{\boldsymbol{\beta}}_{t}^{\prime *} Y_{t-1}$ correspondem a um processo estacionário (valor- $p<0,01$ para o teste DFA - Dickey-Fuller Aumentado).

(a)

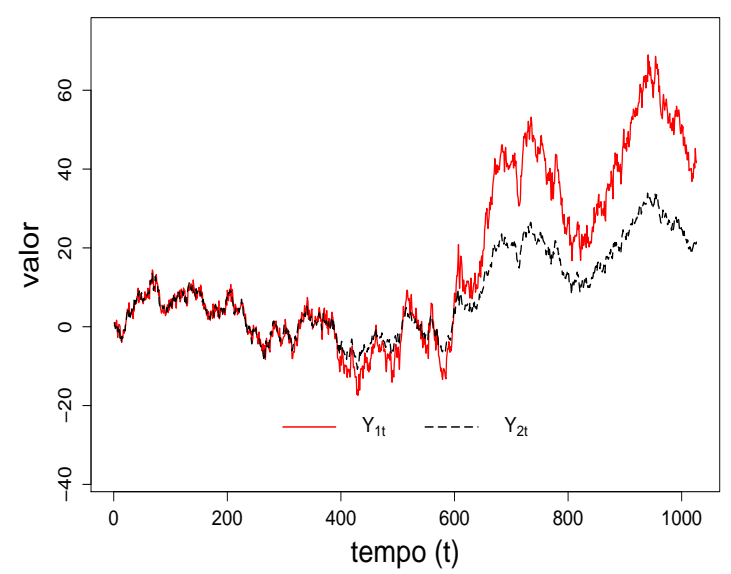

(b)

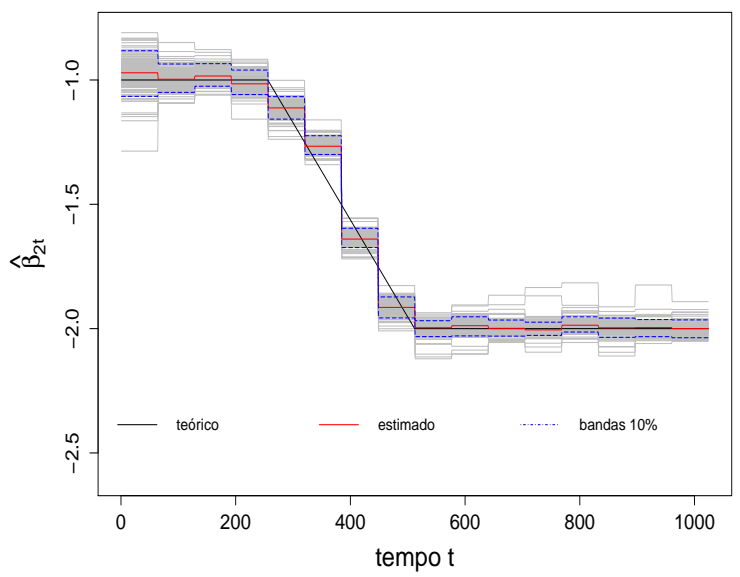

Figura 5.24: Quinto exemplo: (a) Séries cointegradas simuladas $Y_{1 t}$ e $Y_{2 t}$, com $T=1024$, (b) componente teórico $\beta_{2 t}$ de $\boldsymbol{\beta}_{t}=\left[\beta_{1 t} \text { e } \beta_{2 t}\right]^{\prime}$ e estimado $\hat{\beta}_{2 t}$ de $\hat{\boldsymbol{\beta}}_{t}=\left[\hat{\beta}_{1 t} \hat{\beta}_{2 t}\right]^{\prime}$. 
(c)

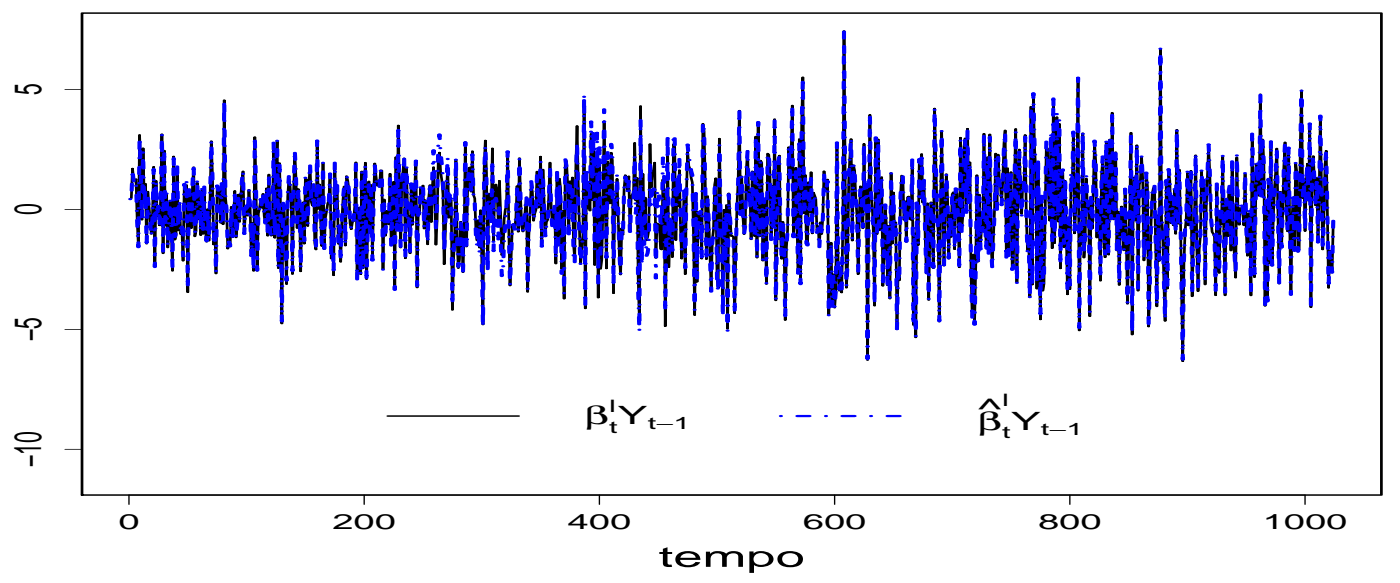

Figura 5.24: Quinto exemplo (continuação): (c) Resíduos de cointegração teóricos $\left(\boldsymbol{\beta}_{t}^{\prime} Y_{t-1}\right)$ e estimados $\left(\hat{\boldsymbol{\beta}}_{t}^{\prime} Y_{t-1}\right)$.

Apresentamos na Figura 5.25 os resíduos $\hat{u}_{1 t}$ e $\hat{u}_{2 t}$ do modelo. O teste de Shapiro-Wilk indica que $\hat{u}_{1 t}$ e $\hat{u}_{2 t}$ parecem ter (individualmente) uma distribuição normal (valores- $p$ iguais a 0,6607 e 0,5273 respectivamente). Conforme esperado, validamos a suposição de normalidade do modelo, visto que o modelo foi simulado sob esta condição. Tal fato é confirmado pelos QQ-plots apresentados em 5.25. Além disso, os resíduos parecem ser não-correlacionados, conforme sugerem os correlogramas apresentados na mesma figura.
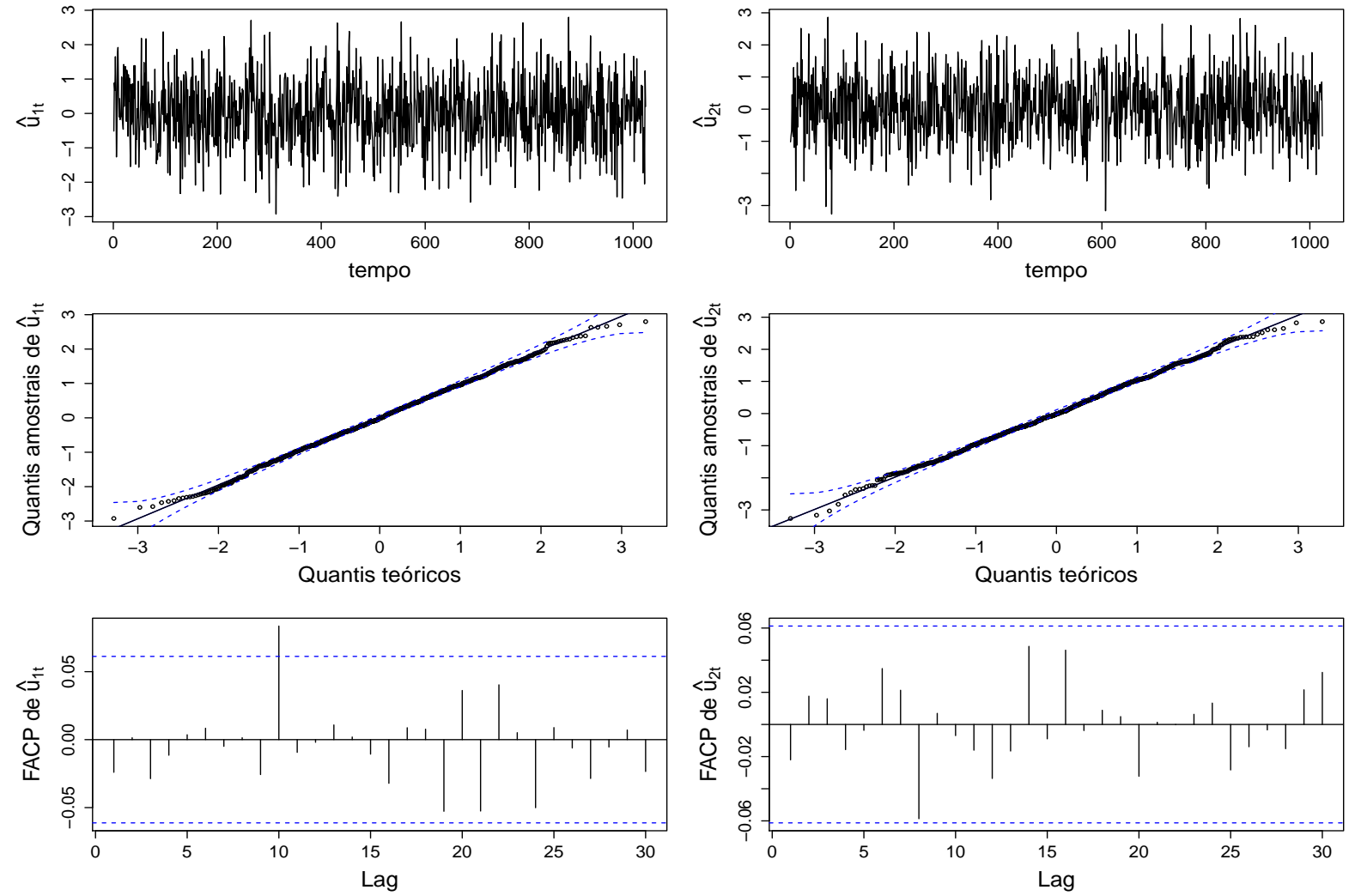

Figura 5.25: Quinto exemplo (completo): Resíduos estimados pelo modelo, quantis amostrais e função de autocorrelação parcial (FACP) de $\hat{u}_{t}=\left[\hat{u}_{1 t}, \hat{u}_{2 t}\right]^{\prime}$. 
Por meio do valor- $p$ bootstrap apresentado na Tabela 5.14, existem evidências que nos levam a rejeitar a hipótese de cointegração constante ao longo do tempo.

Tabela 5.14: Ex.05: Estimativa e medidas bootstrap ${ }^{\star}$ sob H0 (vetor de cointegração constante) para o modelo completo.

\begin{tabular}{c|ccccc}
\hline Parâmetro & Estimativa & Intervalo de confiança $^{\star}$ & Média $^{\star}$ & Erro Padrão $^{\star}$ & valor- $p^{\star}$ \\
\hline$R V_{C V T}^{(J)}(\mathrm{H} 0)$ & 1287,554 & {$[15,7393 ; 50,93115]$} & 30,23741 & 8,4141 & 0,0000 \\
\hline
\end{tabular}

Apresentamos na Tabela 5.15 estimativas dos parâmetros considerados no modelo, bem como medidas bootstrap de interesse. Podemos notar que a maioria das estimativas e as médias bootstrap estão próximas dos valores teóricos dos parâmetros. As excessões são os parâmetros $d_{10}^{*(1,1)}, d_{23}^{*(1,1)}, d_{32}^{*(1,1)}$ e $d_{32}^{*(2,1)}$.

Tabela 5.15: Ex.05: Estimativas e medidas bootstrap* sob HA (transição suave entre duas escalas) para o modelo completo.

\begin{tabular}{c|cccc}
\hline Parâmetro & Estimativa & Intervalo de confiança $^{\star}$ & Média $^{\star}$ & Erro Padrão $^{\star}$ \\
\hline$R V_{C V T}^{(J)}(\mathrm{HA})$ & 1287,55 & {$[1181,31 ; 1802,49]$} & 1535,35 & 158,35 \\
\hline$\alpha_{11}$ & $-1,9299$ & {$[-2,1387 ;-1,7515]$} & $-1,8962$ & 0,4968 \\
$\alpha_{21}$ & 0,0264 & {$[-0,0613 ; 0,0666]$} & 0,0042 & 0,0310 \\
\hline$\sigma_{11}$ & 0,9397 & {$[0,7572 ; 1,0658]$} & 0,9114 & 0,0806 \\
$\sigma_{12}=\sigma_{21}$ & $-0,0191$ & {$[-0,0568 ; 0,0499]$} & $-0,0008$ & 0,0281 \\
$\sigma_{22}$ & 0,9470 & {$[0,7889 ; 1,1584]$} & 0,9556 & 0,0870 \\
\hline$c_{00}^{(1,1)}$ & 0,5194 & {$[0,4637 ; 0,5662]$} & 0,4993 & 0,1248 \\
$c_{00}^{(2,1)}$ & $-0,8327$ & {$[-0,9095 ;-0,7526]$} & $-0,8065$ & 0,2014 \\
\hline$d_{00}^{(1,1)}$ & 0,0134 & {$[-0,0154 ; 0,0170]$} & 0,0007 & 0,0083 \\
$d_{00}^{(2,1)}$ & 0,1772 & {$[0,1600 ; 0,2211]$} & 0,1884 & 0,0483 \\
\hline$d_{10}^{(1,1)}$ & 0,0228 & {$[-0,0186 ; 0,0193]$} & 0,0005 & 0,0097 \\
$d_{10}^{(2,1)}$ & 0,0668 & {$[0,0648 ; 0,1120]$} & 0,0862 & 0,0247 \\
$d_{11}^{(1,1)}$ & 0,0070 & {$[-0,0134 ; 0,0151]$} & 0,0002 & 0,0075 \\
$d_{11}^{(2,1)}$ & $-0,0145$ & {$[-0,0311 ; 0,0263]$} & $-0,0010$ & 0,0149 \\
\hline$d_{20}^{(1,1)}$ & $-0,0086$ & {$[-0,0210 ; 0,0212]$} & 0,0004 & 0,0110 \\
$d_{20}^{(2,1)}$ & 0,0109 & {$[-0,0206 ; 0,0217]$} & 0,0017 & 0,0112 \\
$d_{21}^{(1,1)}$ & $-0,0084$ & {$[-0,0164 ; 0,0188]$} & 0,0006 & 0,0090 \\
$d_{21}^{(2,1)}$ & 0,0847 & {$[0,0431 ; 0,0996]$} & 0,0725 & 0,0221 \\
$d_{22}^{(1,1)}$ & 0,0049 & {$[-0,0164 ; 0,0134]$} & 0,0002 & 0,0074 \\
$d_{22}^{(2,1)}$ & $-0,0086$ & {$[-0,0258 ; 0,0335]$} & 0,0007 & 0,0148 \\
$d_{23}^{(1,1)}$ & 0,0014 & {$[-0,0126 ; 0,0130]$} & $-0,0001$ & 0,0069 \\
$d_{23}^{(2,1)}$ & $-0,0020$ & {$[-0,0261 ; 0,0280]$} & 0,0011 & 0,0139 \\
\hline$d_{30}^{(1,1)}$ & 0,0044 & {$[-0,0255 ; 0,0212]$} & 0,0004 & 0,0116 \\
\hline & & {$[$ Continua na página $38 g u i n t e)$} & \\
\hline
\end{tabular}


Tabela 5.15 - Continuação da página anterior

\begin{tabular}{c|cccc}
\hline Parâmetro & Estimativa & Intervalo de confiança & Média $^{\star}$ & Erro Padrão $^{\star}$ \\
\hline$d_{30}^{(2,1)}$ & $-0,0018$ & {$[-0,0206 ; 0,0279]$} & 0,0019 & 0,0118 \\
$d_{31}^{(1,1)}$ & $-0,0140$ & {$[-0,0242 ; 0,0220]$} & $-0,0004$ & 0,0114 \\
$d_{31}^{(2,1)}$ & 0,0172 & {$[-0,0191 ; 0,0275]$} & 0,0031 & 0,0117 \\
$d_{32}^{(1,1)}$ & $-0,0237$ & {$[-0,0193 ; 0,0236]$} & 0,0005 & 0,0104 \\
$d_{32}^{(2,1)}$ & 0,0414 & {$[-0,0163 ; 0,0378]$} & 0,0128 & 0,0129 \\
$d_{33}^{(1,1)}$ & 0,0002 & {$[-0,0171 ; 0,0151]$} & $-0,0003$ & 0,0079 \\
$d_{33}^{(2,1)}$ & 0,0247 & {$[-0,0098 ; 0,0557]$} & 0,0248 & 0,0154 \\
$d_{34}^{(1,1)}$ & $-0,0013$ & {$[-0,0147 ; 0,0142]$} & 0,0002 & 0,0075 \\
$d_{34}^{(2,1)}$ & 0,0019 & {$[-0,0312 ; 0,0267]$} & $-0,0013$ & 0,0147 \\
$d_{35}^{(1,1)}$ & $-0,0047$ & {$[-0,0134 ; 0,0148]$} & 0,0001 & 0,0074 \\
$d_{35}^{(2,1)}$ & 0,0099 & {$[-0,0314 ; 0,0271]$} & 0,0003 & 0,0149 \\
$d_{36}^{(1,1)}$ & 0,0119 & {$[-0,0153 ; 0,0136]$} & 0,0000 & 0,0072 \\
$d_{36}^{(2,1)}$ & $-0,0228$ & {$[-0,0277 ; 0,0303]$} & 0,0009 & 0,0143 \\
$d_{37}^{(1,1)}$ & $-0,0033$ & {$[-0,0140 ; 0,0154]$} & 0,0003 & 0,0075 \\
$d_{37}^{(2,1)}$ & 0,0070 & {$[-0,0313 ; 0,0274]$} & $-0,0003$ & 0,0149 \\
\hline
\end{tabular}

Apresentamos nas Figuras 5.26, 5.27 e 5.28 os histogramas bootstrap dos parâmetros do modelo. Podemos notar em todas as figuras que os valores teóricos dos parâmetros estão dentro dos intervalos de confiança bootstrap de 95\%. Pelas figuras ainda é possível comparar a estimativa de cada parâmetro do modelo com sua respectiva média boostrap. Apesar de estarem dentro dos intervalos de confiança bootstrap, percebemos um pequeno desvio entre as estimativas e médias bootstrap para o parâmetro $R V_{C V T}^{(J)}$ sob HA.

(a)

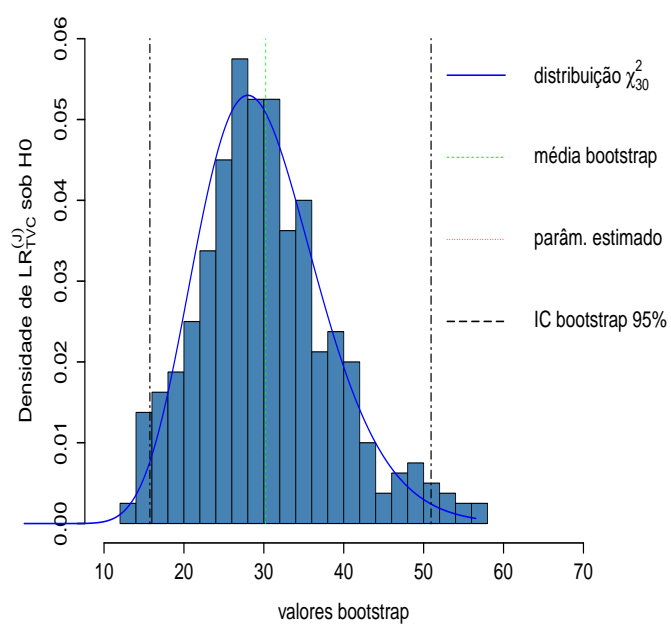

(b)

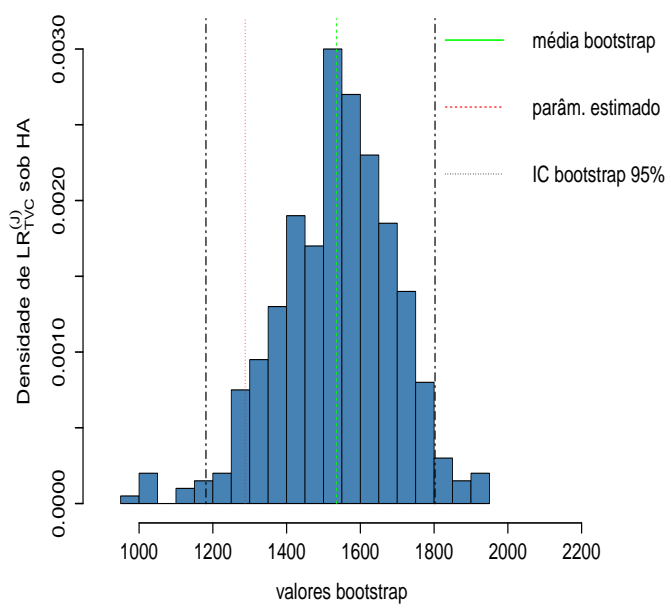

Figura 5.26: Quinto exemplo: Histograma, densidade e quantis bootstrap 2,5\% e 97,5\% para $R V_{C V T}^{(J)}$ sob H0 (transição suave entre duas escalas) (a) e sob HA (transição suave entre dois regimes) (b). 
(a)

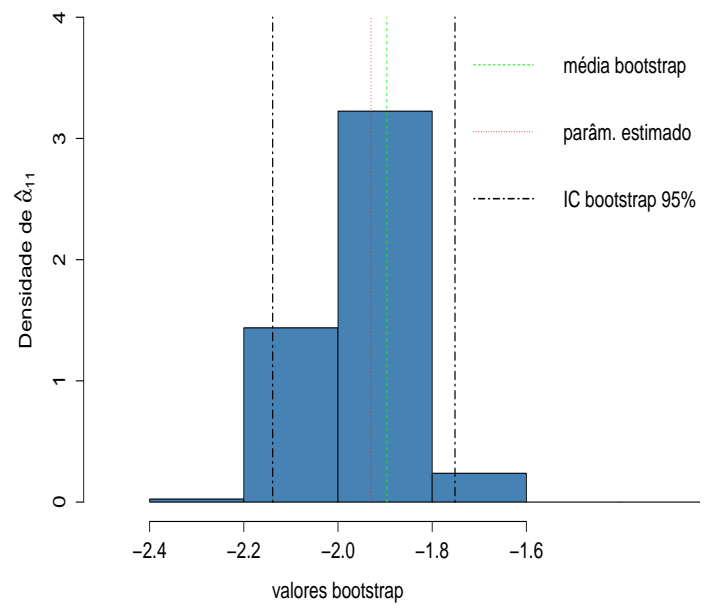

(b)

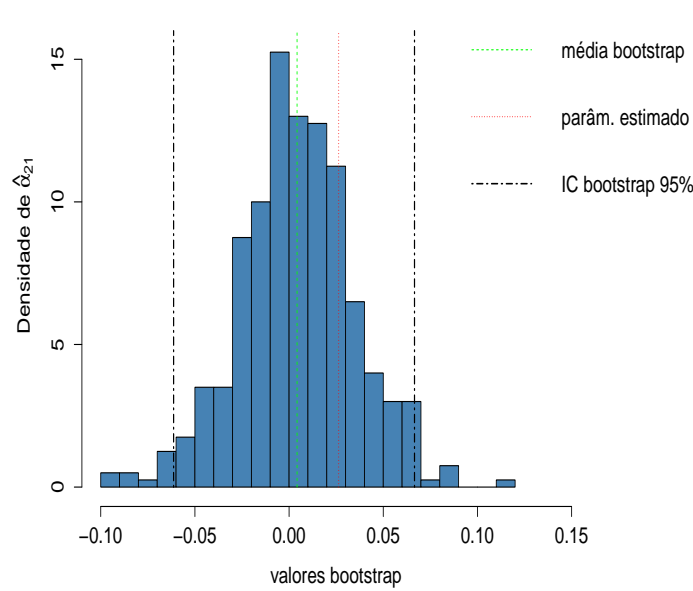

Figura 5.27: Quinto exemplo: Histograma, densidade e quantis bootstrap 2, 5\% e 97, 5\% sob HA

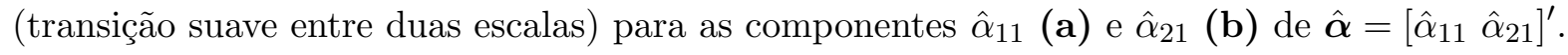

(a)

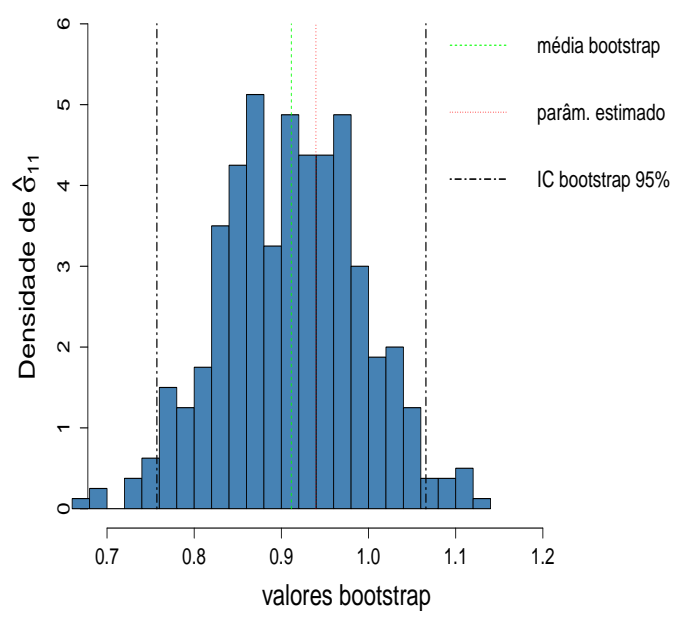

(b)

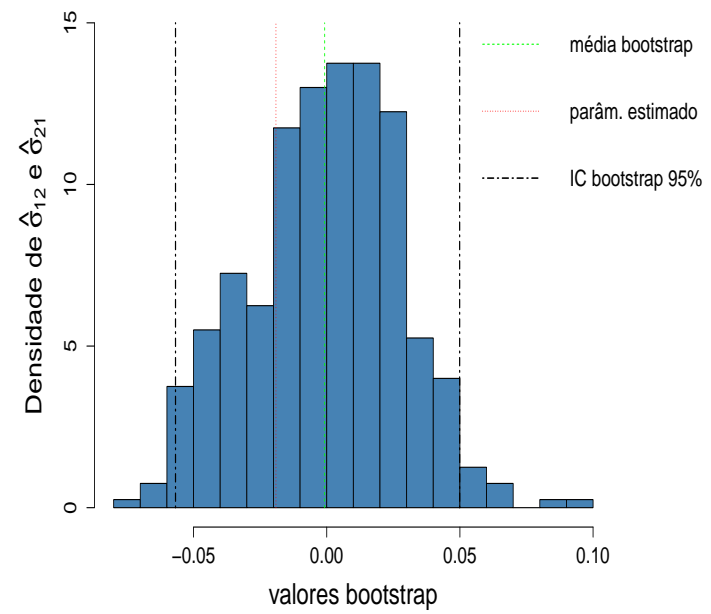

(c)

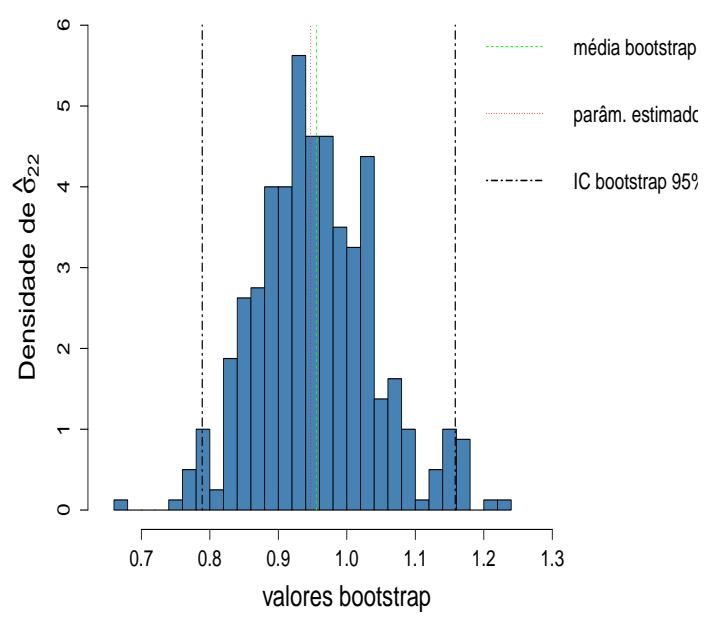

Figura 5.28: Quinto exemplo: Histograma, densidade e quantis bootstrap 2, 5\% e 97, 5\% sob HA (transição suave entre duas escalas) para a componente $\hat{\sigma}_{11}$ (a), componentes $\hat{\sigma}_{12}$ e $\hat{\sigma}_{21}$ (b) e componente $\hat{\sigma}_{22}$ (c) de $\hat{\boldsymbol{\Sigma}}$. 
Vamos considerar a seguir ajustes do modelo para as séries simuladas utilizando as técnicas de limiarização apresentadas na Seção 3.4. A Figura 5.29 apresenta a medida $\theta_{j, k}$ da Equação 3.18, para cada $D_{j k}$. Valores abaixo do limiar (dado pela Equação 3.22) devem ser considerados como candidatos a serem excluídos do modelo. Estamos interessados na característica de que um sinal pode ser suavizado ao ser descrito por um número relativamente pequeno de coeficientes de ondaletas (Morettin, 2014).

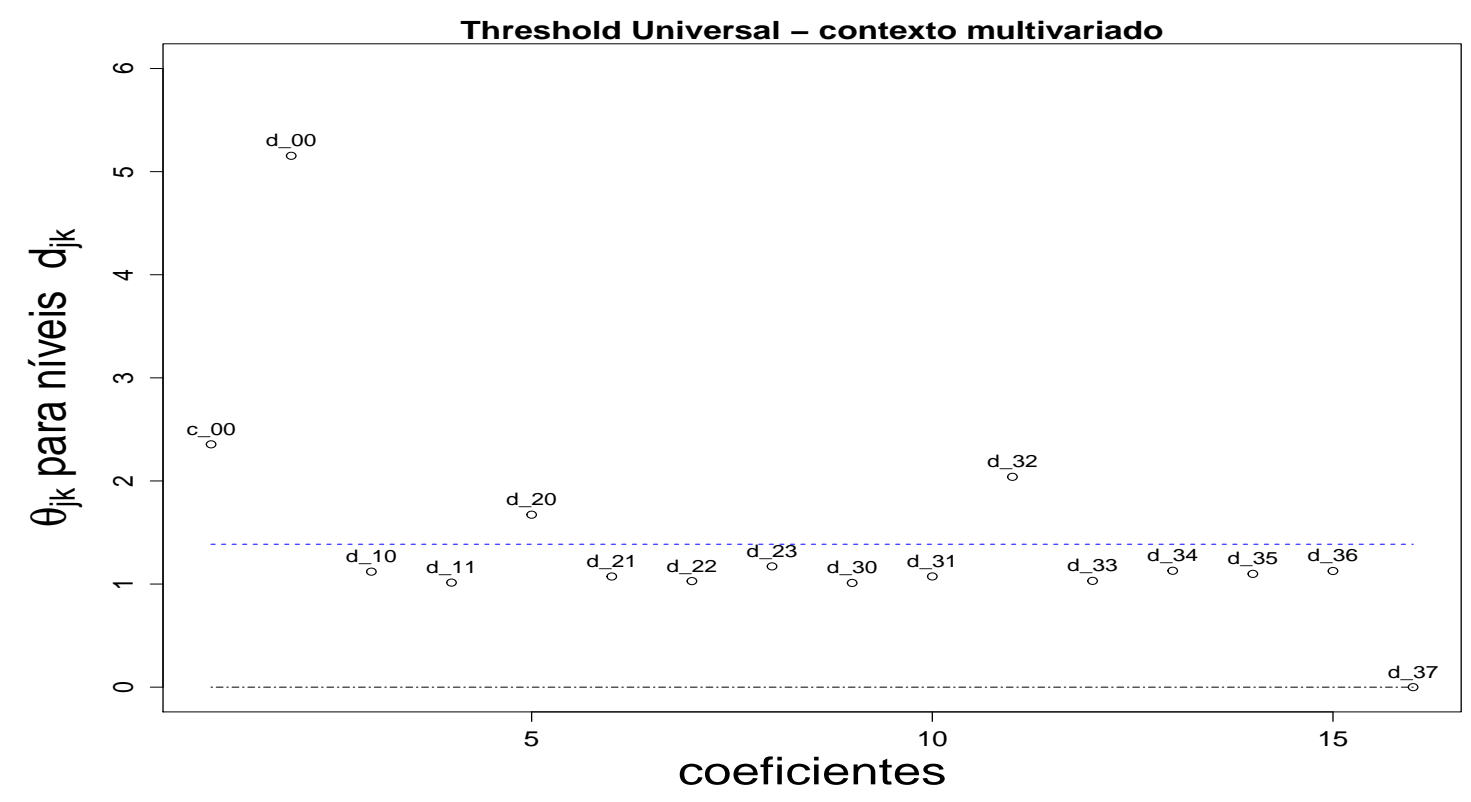

Figura 5.29: Threshold para o modelo completo, considerando $J=3$.

Com base na Figura 5.29, foram considerados modelos em que excluímos, de maneira sequencial, coeficientes de ondaletas do modelo. Para cada modelo considerado, verificamos também o valor atingido para o máximo da log-verossimilhança max $\mathscr{L}\left(\boldsymbol{\theta}_{J}\right)$, definido em 4.32. Estes resultados são descritos na Tabela 5.16. Priorizamos modelos em que ao excluir $\mathbf{d}_{i j}$ 's, não haja grandes variações em $\max \mathscr{L}\left(\boldsymbol{\theta}_{J}\right)$. Pela tabela, notamos que o Modelo 03 é parcimonioso no sentido de permitir a exclusão dos parâmtros sugeridos na Figura 5.29 e não alterar de maneira substancial a $\max \mathscr{L}\left(\boldsymbol{\theta}_{J}\right)$. Note que para os modelos 04 e 05 a variação é maior.

Tabela 5.16: Modelos candidatos: Exclusão sequencial de parâmetros matriciais $\mathbf{d}_{j k}$

\begin{tabular}{cccc}
\hline Modelos & Parâmetros excluídos & máx $\left(\log R V_{C V T}^{(J)}\right)$ & Variação \\
\hline Modelo 01 & nenhum (modelo completo) & $-2868,21$ & $0,00 \%$ \\
Modelo 02 & $\mathbf{d}_{11}, \mathbf{d}_{23}, \mathbf{d}_{30}, \mathbf{d}_{31}, \mathbf{d}_{34}, \mathbf{d}_{35}, \mathbf{d}_{36}, \mathbf{d}_{37}$ & $-2873,28$ & $-0,18 \%$ \\
Modelo 03 & $\mathbf{d}_{11}, \mathbf{d}_{22}, \mathbf{d}_{23}, \mathbf{d}_{30}, \mathbf{d}_{31}, \mathbf{d}_{34}, \mathbf{d}_{35}, \mathbf{d}_{36}, \mathbf{d}_{37}$ & $-2874,24$ & $-0,21 \%$ \\
Modelo 04 & $\mathbf{d}_{11}, \mathbf{d}_{21}, \mathbf{d}_{22}, \mathbf{d}_{23}, \mathbf{d}_{30}, \mathbf{d}_{31}, \mathbf{d}_{34}, \mathbf{d}_{35}, \mathbf{d}_{36}, \mathbf{d}_{37}$ & $-2958,37$ & $-3,14 \%$ \\
Modelo 05 & $\mathbf{d}_{10}, \mathbf{d}_{11}, \mathbf{d}_{21}, \mathbf{d}_{22}, \mathbf{d}_{23}, \mathbf{d}_{30}, \mathbf{d}_{31}, \mathbf{d}_{33}, \mathbf{d}_{34}, \mathbf{d}_{35}, \mathbf{d}_{36}, \mathbf{d}_{37}$ & $-3176,94$ & $-10,76 \%$ \\
\hline
\end{tabular}

Além disso, podemos notar pela Figura 5.30 que os Modelos 02 e 03 apresentam vetores de cointegração estimados semelhantes ao modelo completo. O mesmo não acontece para os modelos 04 e 05 . 
(a) Modelo 01 (completo)

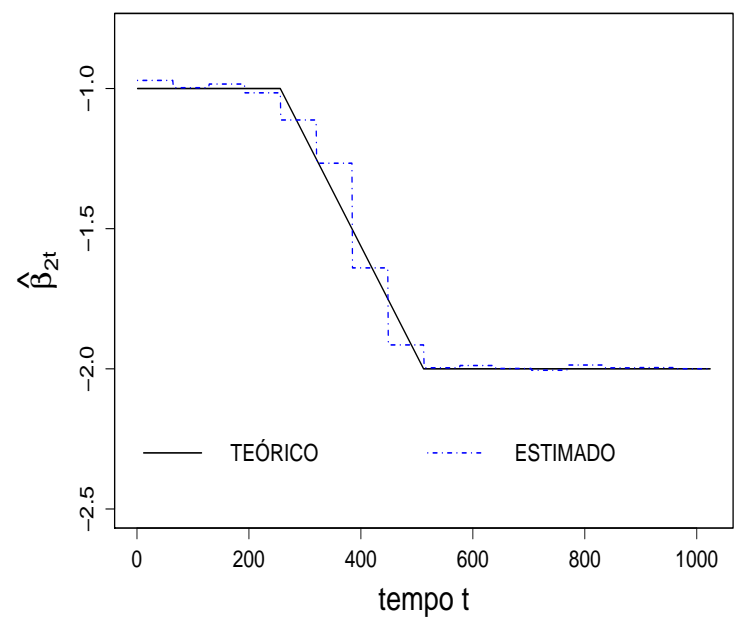

(c) Modelo 03

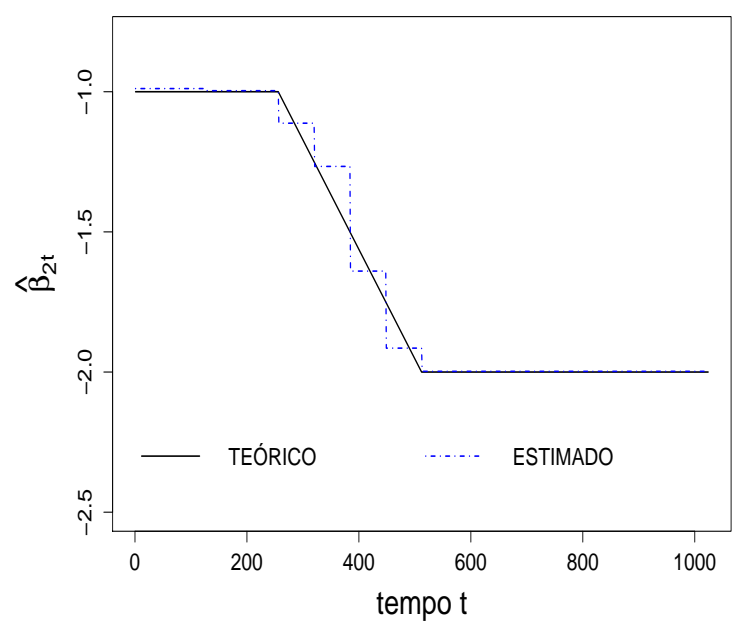

(e) Modelo 05

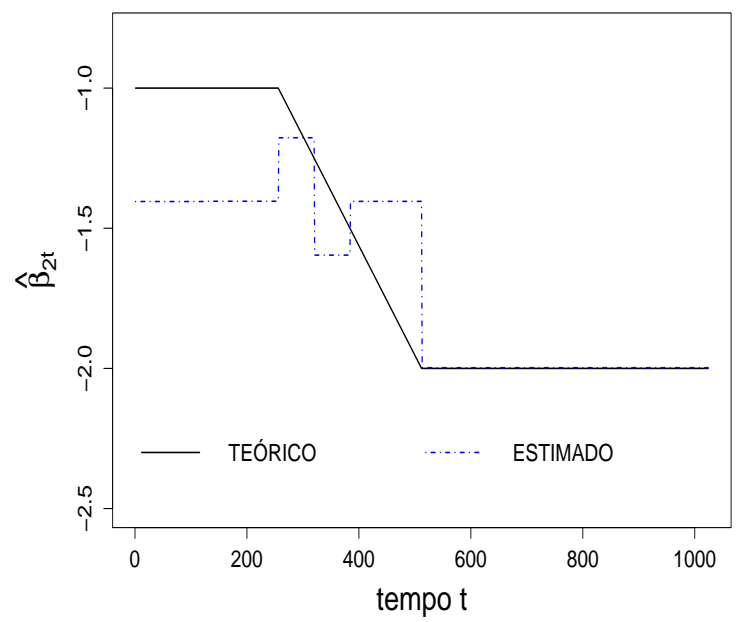

(b) Modelo 02

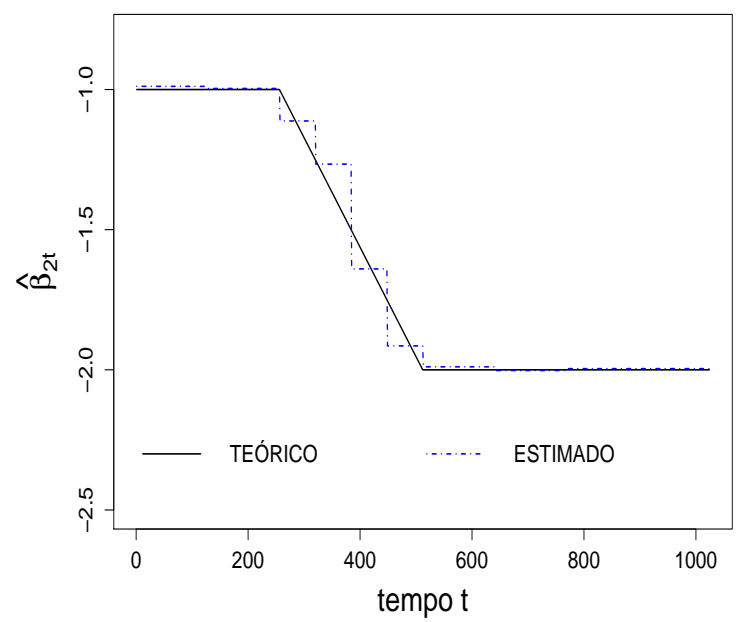

(d) Modelo 04

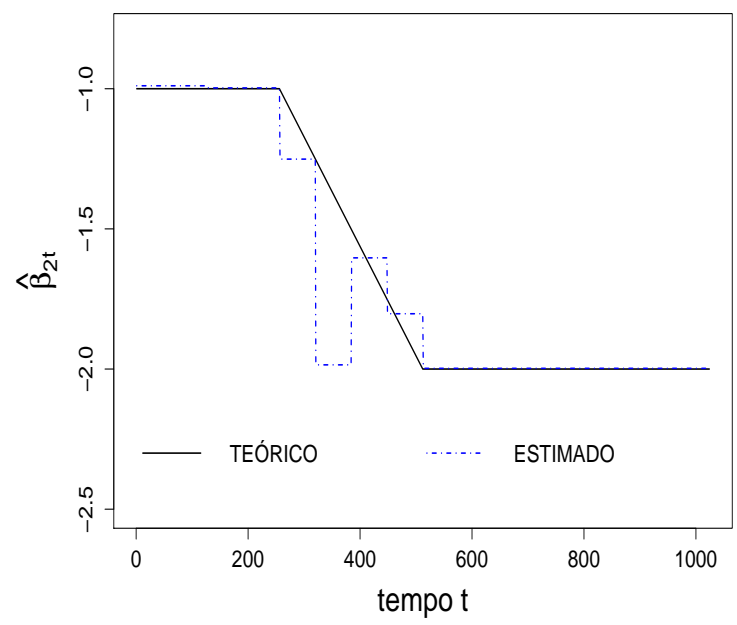

Figura 5.30: Componente $\hat{\beta}_{2 t}$ de $\hat{\beta}_{t}=\left(\hat{\beta}_{1 t}, \hat{\beta}_{2 t}\right)$ e teórico $\beta_{2 t}$ para referência: Modelos 01, 02, 03, 04 e 05 considerados. 
Com base na discussão subjacente, para o Modelo 03 (limiarizado), apresentamos a Figura 5.31 com $\beta_{2 t}$ teórico, juntamente com sua estimativa e bandas de confiança bootstrap de $95 \%$ (a). Em (b), apresentamos os resíduos de cointegração teóricos e estimados.

(a)

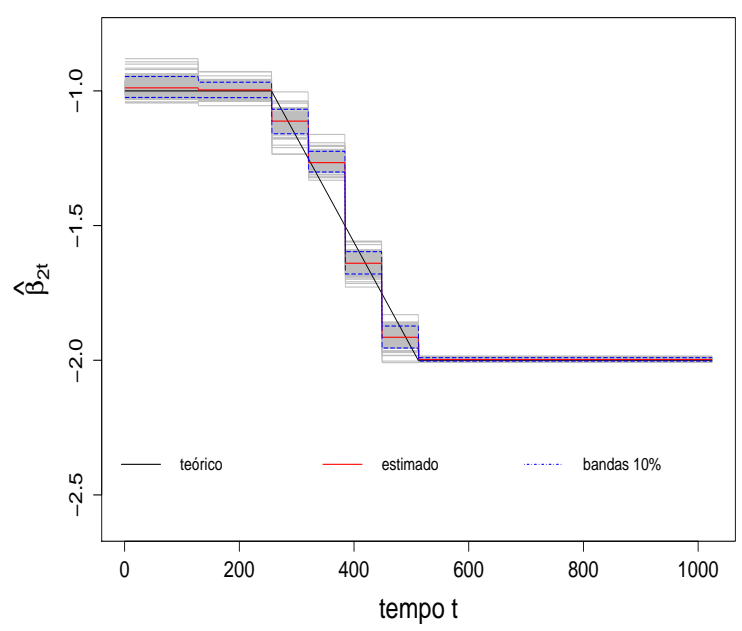

(b)

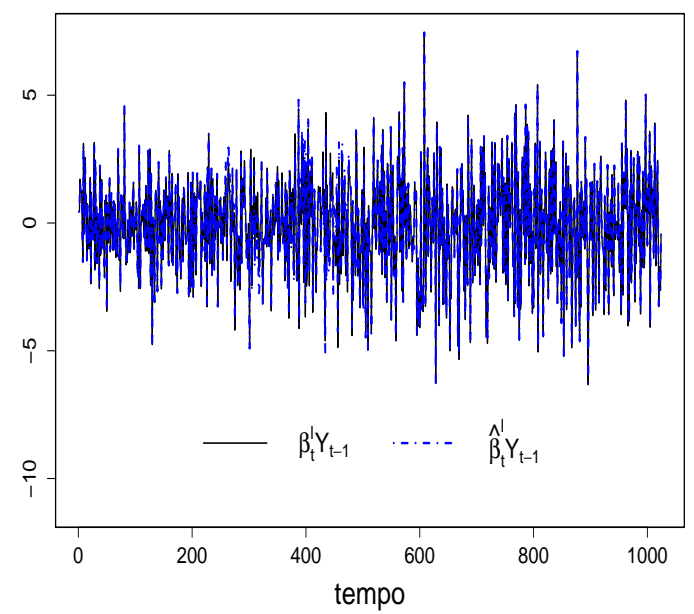

Figura 5.31: (a) Componente $\hat{\beta}_{2 t}$ de $\hat{\beta}_{t}=\left(\hat{\beta}_{1 t}, \hat{\beta}_{2 t}\right)$ e teórico $\beta_{2 t}$ para referência $(J=3)$, (b) Resíduos de cointegração teóricos $\left(\beta_{t}^{\prime} Y_{t-1}\right)$ e estimados $\left(\hat{\beta}_{t}^{\prime} Y_{t-1}\right)$.

Verificamos que os resíduos de cointegração estimados $\hat{\boldsymbol{\beta}}_{t}^{\prime} Y_{t-1}$, apresentados na Figura 5.31 (c), correspondem a um processo estacionário (valor- $p<0,01$ para o teste $D F A$ ).

Ao aplicarmos o teste de Shapiro-Wilk individualmente aos resíduos $u_{1 t}$ e $u_{2 t}$, apresentados na Figura 5.32, verificamos que eles parecem ter uma distribuição normal (valores- $p$ iguais a 0,5317 e 0,5484 respectivamente). Conforme esperado, validamos a suposição de normalidade do modelo, visto que o modelo foi simulado sob esta condição. Tal fato é confirmado pelos QQ-plots apresentados em 5.32. Além disso, os resíduos $u_{1 t}$ e $u_{2 t}$ parecem ser não-correlacionados, conforme sugerem os correlogramas também apresentados em 5.32. 

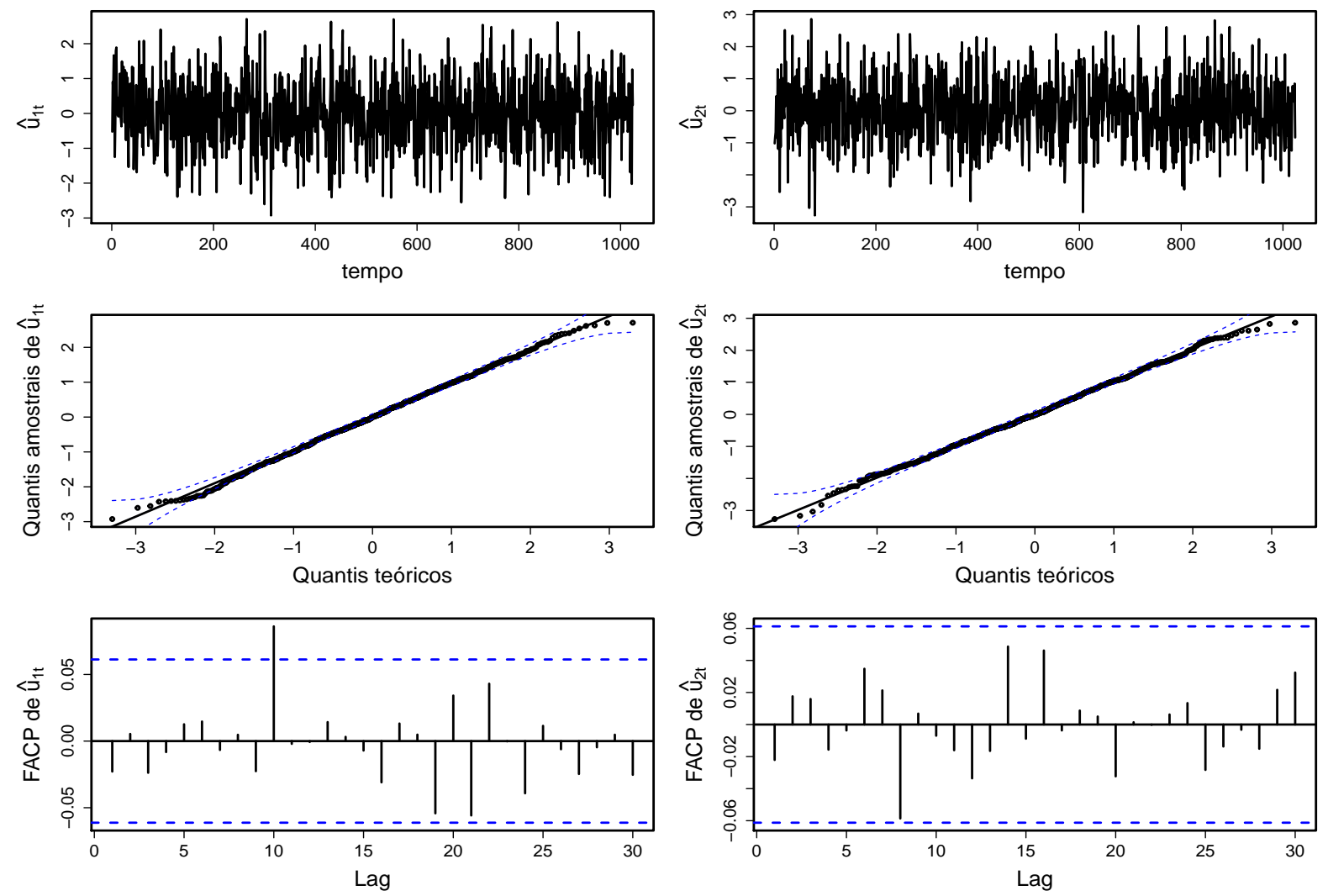

Figura 5.32: Quinto exemplo (limiarizado): Resíduos estimados pelo modelo, quantis amostrais e função de autocorrelação parcial (FACP) de $\hat{u}_{t}=\left[\hat{u}_{1 t}, \hat{u}_{2 t}\right]^{\prime}$.

Por meio do valor- $p$ bootstrap apresentado na Tabela 5.17, existem evidências que nos levam a rejeitar a hipótese de cointegração constante ao longo do tempo. Consequentemente, aplicar a limiarização não alterou a decisão já tomada para o modelo completo.

Tabela 5.17: Ex.05: Resultados bootstrap sob H0 (vetor de cointegração constante) para o modelo limiarizado.

\begin{tabular}{c|ccccc}
\hline Parâmetro & Estimado & Intervalo de confiança & Média & Erro Padrão & valor- $p$ \\
\hline$R V_{C V T}^{(J)}(\mathrm{H} 0)$ & 1275,49 & {$[4,5427 ; 23,2584]$} & 11,9564 & 5,0359 & 0,0000 \\
\hline
\end{tabular}

A Tabela 5.18 por sua vez apresenta as estimativas dos parâmetros, bem como medidas bootstrap de interesse. Note que os parâmetros que não foram excluídos do modelo foram reestimados considerando a ausência dos demais.

Tabela 5.18: Ex.05: Estimativas e medidas bootstrap* sob HA (transição suave entre duas escalas) para o modelo limiarizado.

\begin{tabular}{c|cccc}
\hline Parâmetro & Estimado & Intervalo de confiança* & Média* $^{*}$ & Erro Padrão* $^{*}$ \\
\hline$R V_{C V T}^{(J)}(\mathrm{HA})$ & 1275,49 & {$[1157,23 ; 1778,75]$} & 1510,58 & 158,29 \\
\hline$\alpha_{11}$ & $-1,9270$ & {$[-2,1430 ;-1,7782]$} & $-1,9572$ & 0,0929 \\
$\alpha_{21}$ & 0,0263 & {$[-0,0589 ; 0,0642]$} & 0,0038 & 0,0308 \\
\hline \multicolumn{5}{c}{ (Continua na página seguinte) }
\end{tabular}


Tabela 5.18 - Continuação da página anterior

\begin{tabular}{c|cccc}
\hline Parâmetro & Estimado & Intervalo de confiança* & Média $^{*}$ & Erro Padrão* $^{*}$ \\
\hline$\sigma_{11}$ & 0,9508 & {$[0,7806 ; 1,1003]$} & 0,9389 & 0,0821 \\
$\sigma_{12}=\sigma_{21}$ & $-0,0194$ & {$[-0,0609 ; 0,0497]$} & $-0,0007$ & 0,0287 \\
$\sigma_{22}$ & 0,9649 & {$[0,8100 ; 1,1753]$} & 0,9731 & 0,0882 \\
\hline$c_{00}^{(1,1)}$ & 0,5206 & {$[0,4670 ; 0,5633]$} & 0,5131 & 0,0234 \\
$c_{00}^{(2,1)}$ & $-0,8351$ & {$[-0,9090 ;-0,7566]$} & $-0,8293$ & 0,0368 \\
\hline$d_{00}^{(1,1)}$ & 0,0130 & {$[-0,0151 ; 0,0175]$} & 0,0012 & 0,0085 \\
$d_{00}^{(2,1)}$ & 0,1787 & {$[0,1674 ; 0,2196]$} & 0,1931 & 0,0143 \\
\hline$d_{10}^{(1,1)}$ & 0,0228 & {$[-0,0185 ; 0,0177]$} & 0,0000 & 0,0096 \\
$d_{10}^{(2,1)}$ & 0,0670 & {$[0,0661 ; 0,1114]$} & 0,0892 & 0,0118 \\
\hline$d_{20}^{(1,1)}$ & $-0,0103$ & {$[-0,0218 ; 0,0202]$} & 0,0005 & 0,0111 \\
$d_{20}^{(2,1)}$ & 0,0113 & {$[-0,0216 ; 0,0221]$} & 0,0006 & 0,0112 \\
$d_{21}^{(1,1)}$ & $-0,0084$ & {$[-0,0175 ; 0,0183]$} & 0,0005 & 0,0091 \\
$d_{21}^{(2,1)}$ & 0,0848 & {$[0,0469 ; 0,1010]$} & 0,0748 & 0,0132 \\
\hline$d_{32}^{(1,1)}$ & $-0,0237$ & {$[-0,0198 ; 0,0231]$} & 0,0005 & 0,0102 \\
$d_{32}^{(2,1)}$ & 0,0414 & {$[-0,0158 ; 0,0379]$} & 0,0132 & 0,0124 \\
$d_{33}^{(1,1)}$ & 0,0002 & {$[-0,0164 ; 0,0146]$} & $-0,0003$ & 0,0079 \\
$d_{33}^{(2,1)}$ & 0,0247 & {$[-0,0007 ; 0,0546]$} & 0,0255 & 0,0142 \\
\hline
\end{tabular}

Apresentamos nas Figuras 5.33, 5.34 e 5.35 os histogramas bootstrap dos parâmetros do modelo. Mais uma vez, podemos notar em todas as figuras que os valores teóricos dos parâmetros estão dentro dos intervalos de confiança bootstrap de $95 \%$.

(a)

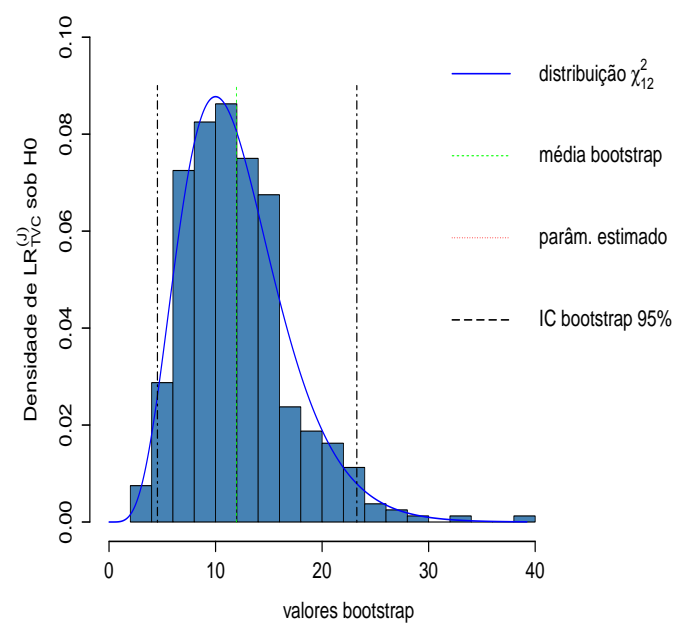

(b)

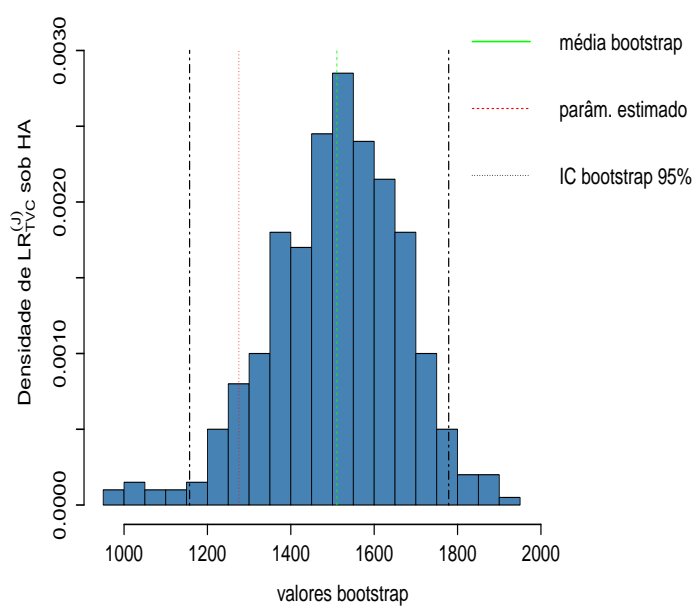

Figura 5.33: Quinto exemplo (limiarizado): Histograma, densidade e quantis bootstrap 2, 5\% e $97,5 \%$ para $R V_{C V T}^{(J)}$ sob H0 (vetor de cointegração constante) (a) e sob HA (transição suave entre dois regimes) (b). 
(a)

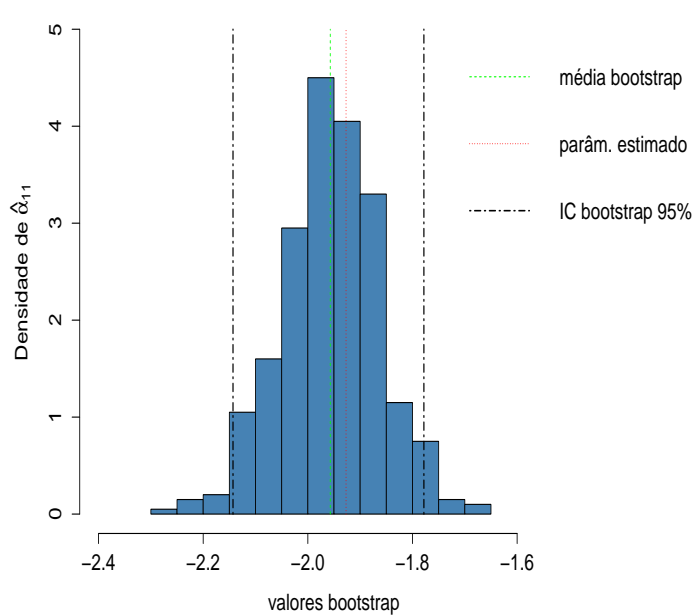

(b)

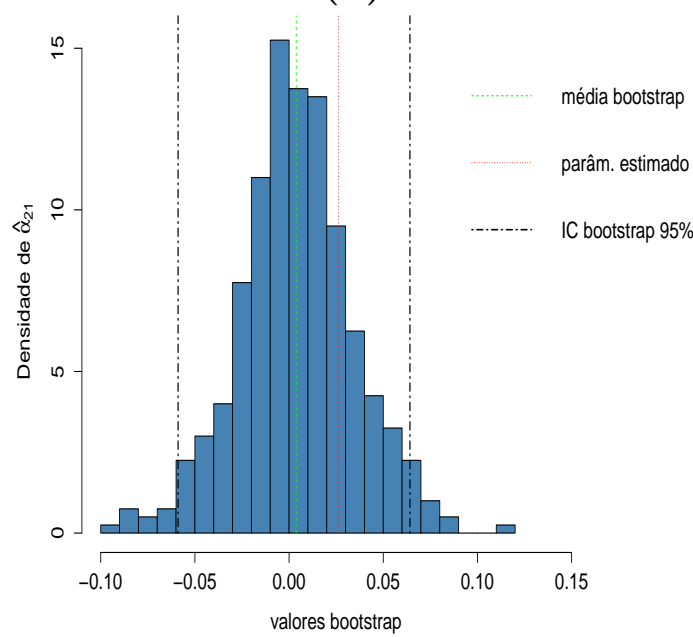

Figura 5.34: Quinto exemplo (limiarizado): Histograma, densidade e quantis bootstrap 2,5\% e $97,5 \%$ para as componentes $\hat{\alpha}_{11}$ (a) e $\hat{\alpha}_{21}$ (b) de $\hat{\boldsymbol{\alpha}}=\left[\begin{array}{ll}\hat{\alpha}_{11} & \hat{\alpha}_{21}\end{array}\right]^{\prime}$.

(a)

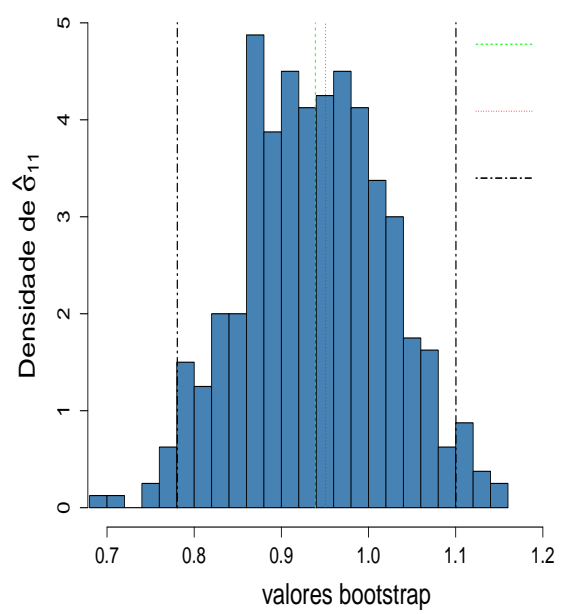

(b)

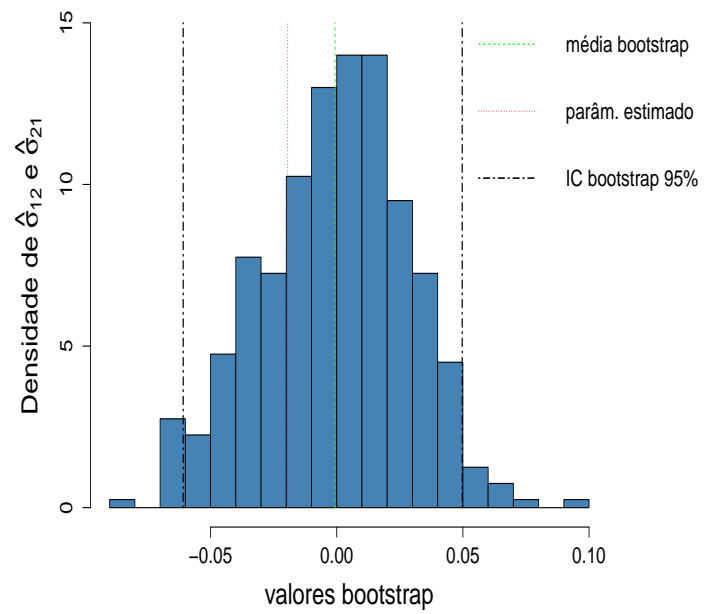

(c)

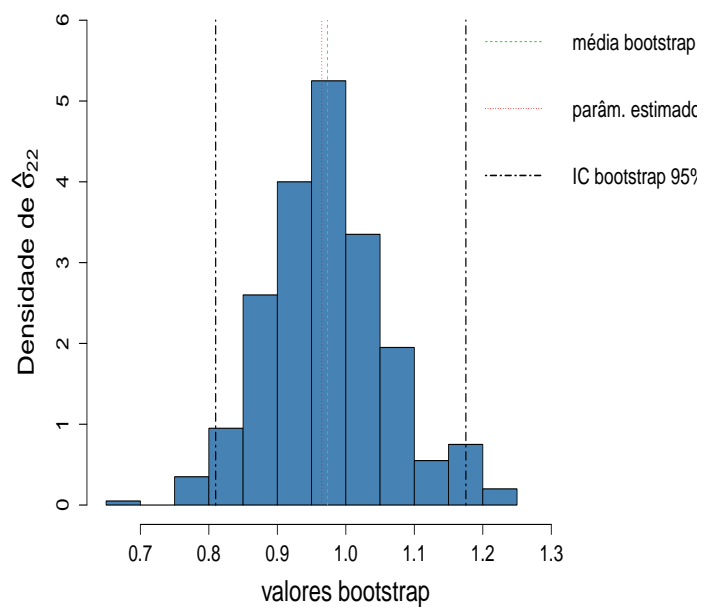

Figura 5.35: Quinto exemplo (limiarizado): Histograma, densidade e quantis bootstrap 2,5\% e $97,5 \%$ sob HA (transição suave entre duas escalas) para a componente $\hat{\sigma}_{11}$ (a), componentes $\hat{\sigma}_{12}$ e $\hat{\sigma}_{21}(\mathbf{b})$ e componente $\hat{\sigma}_{22}$ (c) de $\hat{\boldsymbol{\Sigma}}$. 


\subsection{Exemplo com defasagem}

\subsubsection{Sexto exemplo: Decrescimento logarítmico com Symmlet $S 8$ (considerando defasagem do modelo)}

Nesta simulação, consideramos a componente teórica $\beta_{2 t}^{*}$ de $\boldsymbol{\beta}_{t}^{*}=\left[\begin{array}{ll}\beta_{1 t}^{*} & \beta_{2 t}^{*}\end{array}\right]^{\prime}$ descrevendo um decrescimento logarítmico ao longo tempo, com $t=1, \ldots, T$ e $T=1024$. Para isso, consideramos o vetor de cointegração teórico:

$$
\boldsymbol{\beta}_{t}^{*}=\left[\begin{array}{c}
\beta_{1 t}^{*} \\
\beta_{2 t}^{*}
\end{array}\right]=\left[\begin{array}{c}
1 \\
-\ln \left(\frac{t}{16}+32\right)
\end{array}\right],
$$

Utilizando a expressão 4.1 e $J=5$, podemos escrever o vetor $\boldsymbol{\beta}_{t}^{*}$ por meio da seguinte expressão:

$$
\boldsymbol{\beta}_{t}^{*}=\phi(t) \mathbf{c}^{*}+\psi_{0,0}(t) \mathbf{d}_{0,0}^{*}+\psi_{1,0}(t) \mathbf{d}_{1,0}^{*}+\psi_{1,1}(t) \mathbf{d}_{1,1}^{*}+\ldots+\psi_{5,31}(t) \mathbf{d}_{5,31}^{*}
$$

Por 4.2 temos que $\mathbf{c}^{*}=\left[\begin{array}{lll}c_{0,0}^{*(1,1)} & c_{0,0}^{*(2,1)}\end{array}\right]^{\prime}$ e $\mathbf{d}_{J, 2^{J}-1}^{*}=\left[\begin{array}{ll}\hat{d}_{J, 2^{J}-1}^{*(1,1)} & \hat{d}_{J, 2^{J}-1}^{*(2,1)}\end{array}\right]^{\prime}$, cujos elementos são estimados por $\hat{\boldsymbol{W}}_{J}^{*}$ de 4.68 . Além disso, $\phi(t)$ e $\psi_{0,0}(t)$ são funções da base de ondaletas Symmlet $S 8$.

Apresentamos na Figura 5.36 (a) os gráficos das séries cointegradas simuladas $Y_{1 t}$ e $Y_{2 t}$. Para estas séries, apresentamos em 5.36 (b) o segundo componente dos vetores de cointegração teórico $\left(\beta_{2 t}^{*}\right)$ e estimado $\left(\hat{\beta}_{2 t}^{*}\right)$. Pela figura, é possível notar que $\hat{\beta}_{2 t}^{*}$ está próximo de $\beta_{2 t}^{*}$, sendo que ambos estão dentro das bandas de confiança de $95 \%$, com desvio apenas no início de $\beta_{2 t}^{*}$. Além disso, apresentamos as estimativas dos parâmetros do modelo, juntamente com os valores teóricos em parênteses:

$$
\hat{\boldsymbol{\alpha}}^{*}=\left[\begin{array}{c}
-1,0024 \\
(-1,0000) \\
0,0003 \\
(0,0000)
\end{array}\right], \hat{\boldsymbol{\Sigma}}_{u}^{*}=\left[\begin{array}{cc}
1,0310 & -0,0073 \\
(1,0000) & (0,0000) \\
-0,0073 & \underset{1}{0,9860} \\
(0,0000) & (1,0000)
\end{array}\right] \text { e } \boldsymbol{\Gamma}_{1}=\left[\begin{array}{cc}
0,4971 & 0,4432 \\
(0,5000) & (0,5000) \\
0,0052 & 0,4633 \\
(0,5000) & (0,5000)
\end{array}\right]
$$

Apresentamos na Figura 5.36 (c) os resíduos de cointegração teóricos $\left(\boldsymbol{\beta}_{t}^{*^{\prime}} Y_{t-1}\right)$ e estimados $\left(\hat{\boldsymbol{\beta}}_{t}^{*^{\prime}} Y_{t-1}\right)$. Verificamos que $\hat{\boldsymbol{\beta}}_{t}^{*} Y_{t-1}$ correspondem a um processo estacionário (valor$p<0,01$ para o teste DFA - Dickey-Fuller Aumentado). 
(a)

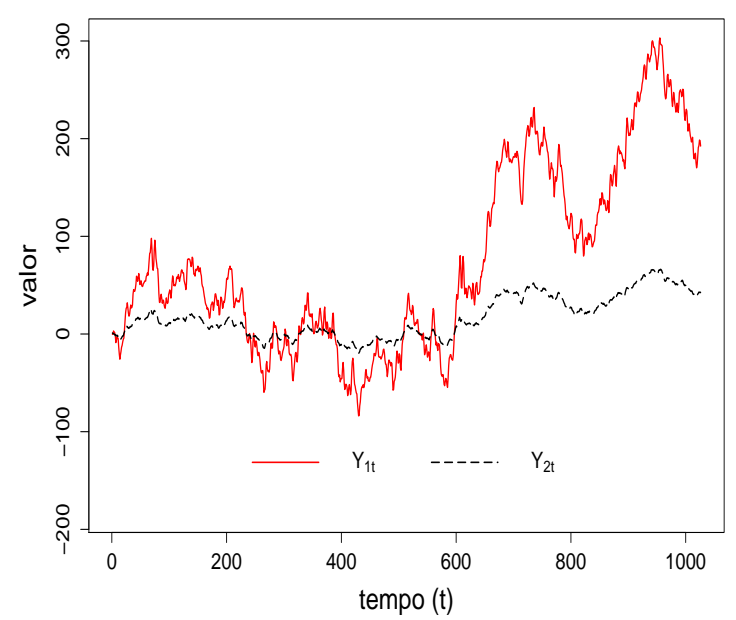

(b)

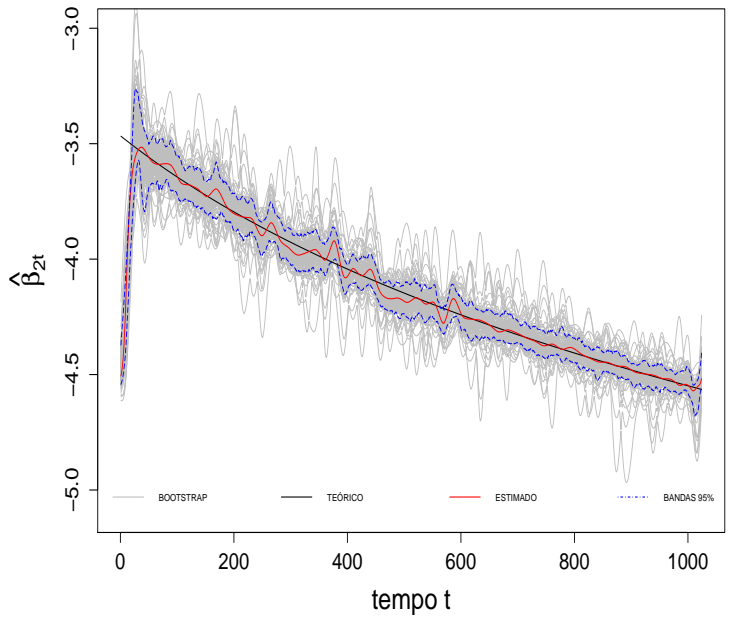

(c)

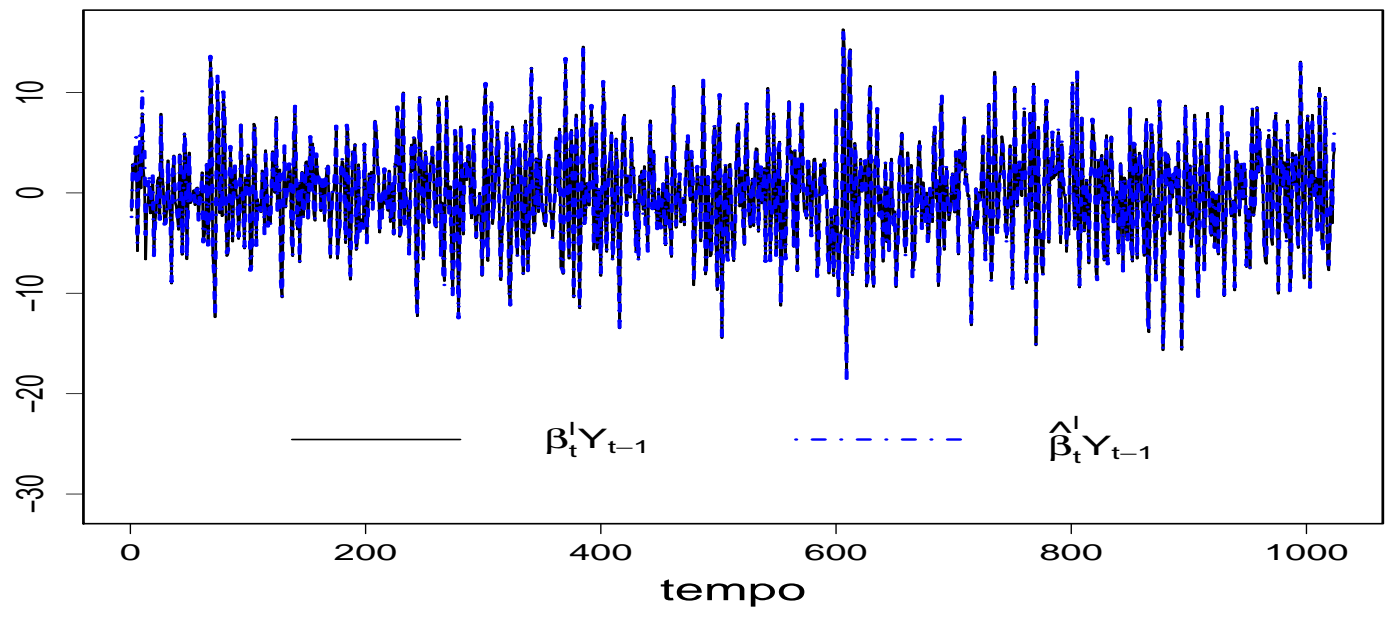

Figura 5.36: Sexto exemplo: (a) Séries cointegradas simuladas $Y_{1 t}$ e $Y_{2 t}$, com $T=1024$, (b)

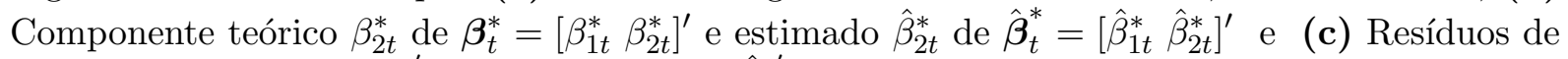
cointegração teóricos $\left(\beta_{t}^{*^{\prime}} Y_{t-1}\right)$ e estimados $\left(\hat{\beta}_{t}^{*^{\prime}} Y_{t-1}\right)$.

Apresentamos na Figura 5.37 os resíduos $\hat{u}_{1 t}$ e $\hat{u}_{2 t}$ do modelo. Ao aplicarmos o teste de Shapiro-Wilk individualmente a $\hat{u}_{1 t}$ e $\hat{u}_{2 t}$, verificamos que eles parecem ter uma distribuição normal (valores- $p$ iguais a 0,1840 e 0,5808 respectivamente). Conforme esperado, validamos a suposição de normalidade do modelo, visto que o modelo foi simulado sob esta condição. Tal fato é confirmado pelos QQ-plots apresentados em 5.37. Além disso, os resíduos parecem ser não-correlacionados, conforme sugerem os correlogramas apresentados na mesma figura. 

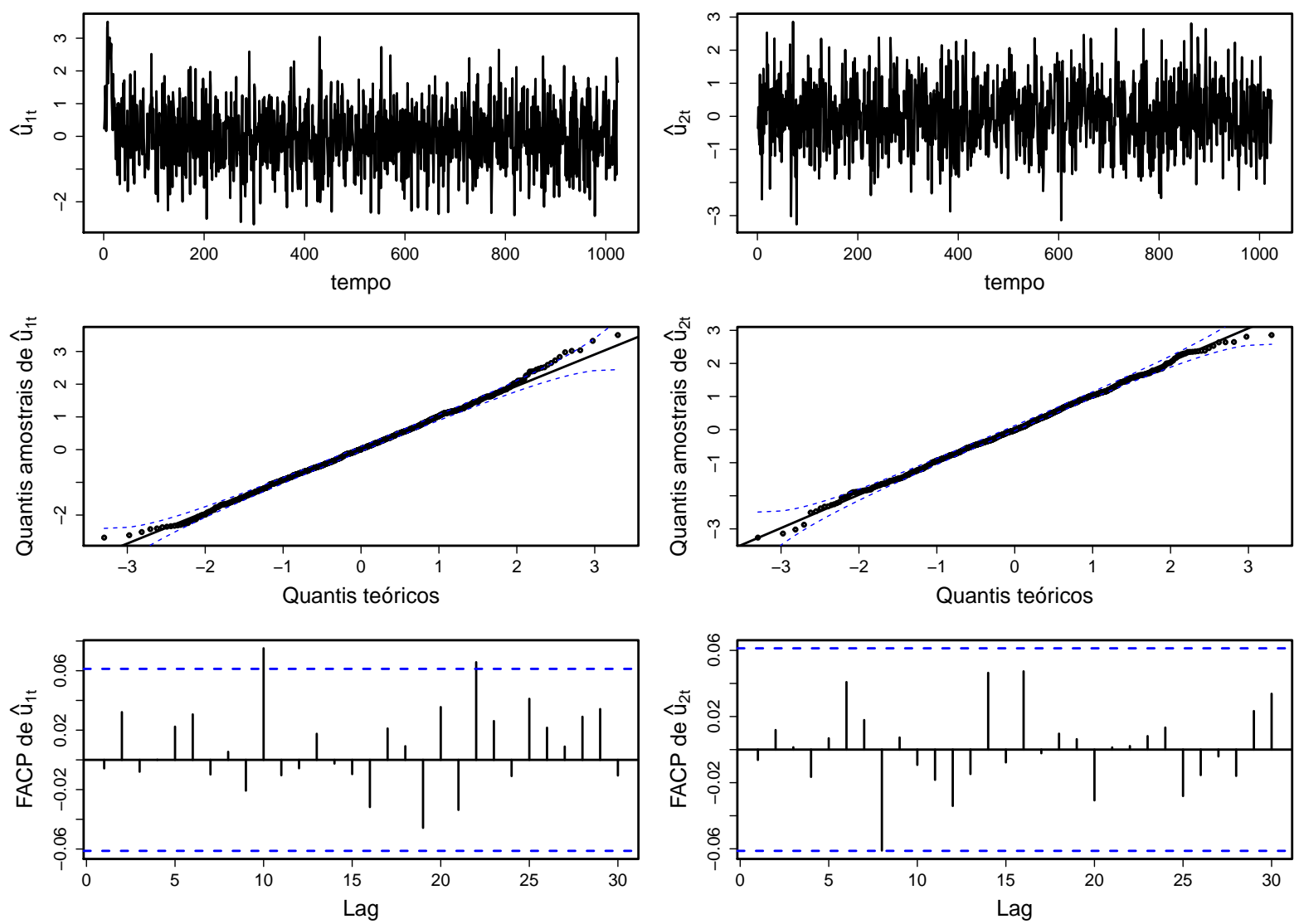

Figura 5.37: Quarto exemplo: Resíduos estimados pelo modelo, quantis amostrais e função de autocorrelação parcial (FACP) de $\hat{u}_{t}=\left[\hat{u}_{1 t}, \hat{u}_{2 t}\right]^{\prime}$.

Por meio do valor- $p$ bootstrap apresentado na Tabela 5.19, existem evidências que nos levam a rejeitar a hipótese de cointegração constante ao longo do tempo.

Tabela 5.19: Ex.06: Estimativa e medidas bootstrap ${ }^{\star}$ sob H0 (vetor de cointegração constante).

\begin{tabular}{c|ccccc}
\hline Parâmetro & Estimativa & Intervalo de confiança & Média $^{*}$ & Erro Padrão $^{*}$ & valor- $p^{*}$ \\
\hline$R V_{C V T}^{(J)}(\mathrm{H} 0)$ & 2210,08 & {$[89,0614 ; 147,5431]$} & 117,6828 & 15,6767 & 0,0000 \\
\hline
\end{tabular}

Apresentamos nas Figuras 5.38, 5.39, 5.40 e 5.41 os histogramas bootstrap dos parâmetros do modelo. Podemos notar em todas as figuras que os valores teóricos dos parâmetros estão dentro dos intervalos de confiança bootstrap de 95\%. Pelas figuras ainda é possível comparar a estimativa de cada parâmetro do modelo com sua respectiva média boostrap. 
(a)

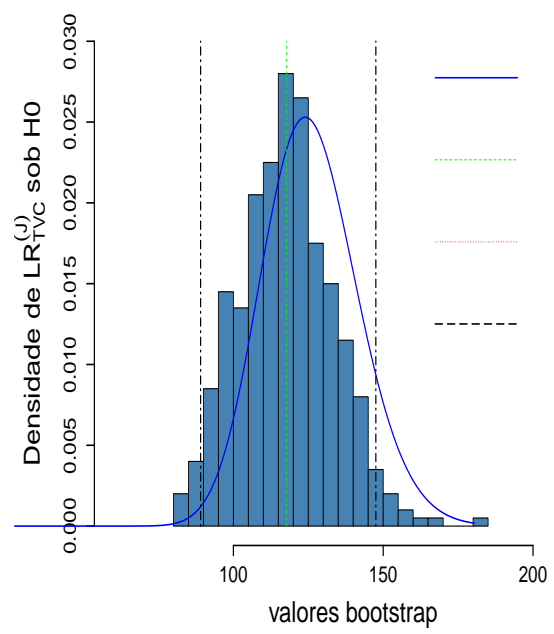

(b)

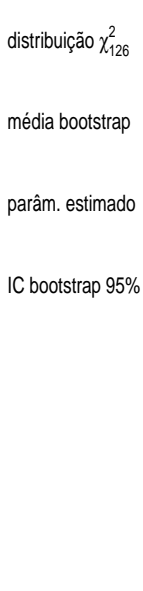

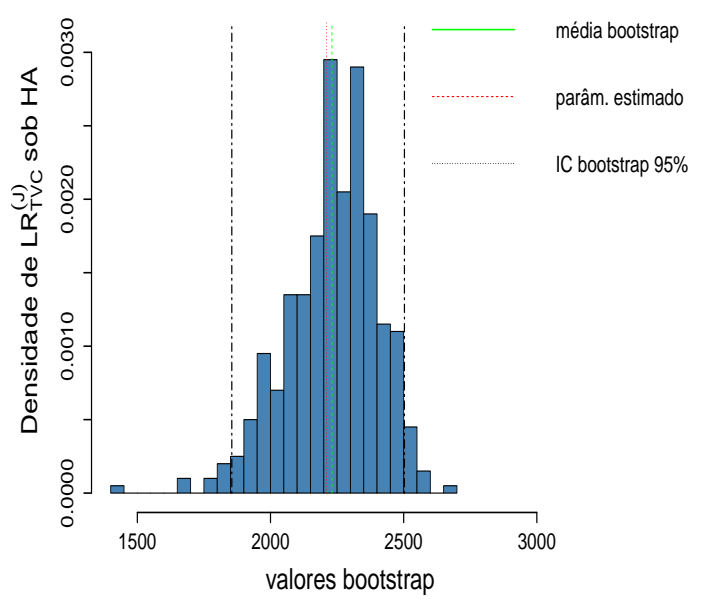

Figura 5.38: Sexto exemplo: Histograma, densidade e quantis bootstrap 2,5\% e 97,5\% para $R V_{C V T}^{(J)}$ sob H0 (vetor de cointegração constante) (a) e sob HA (decrescimento logarítmico) (b).

(a)

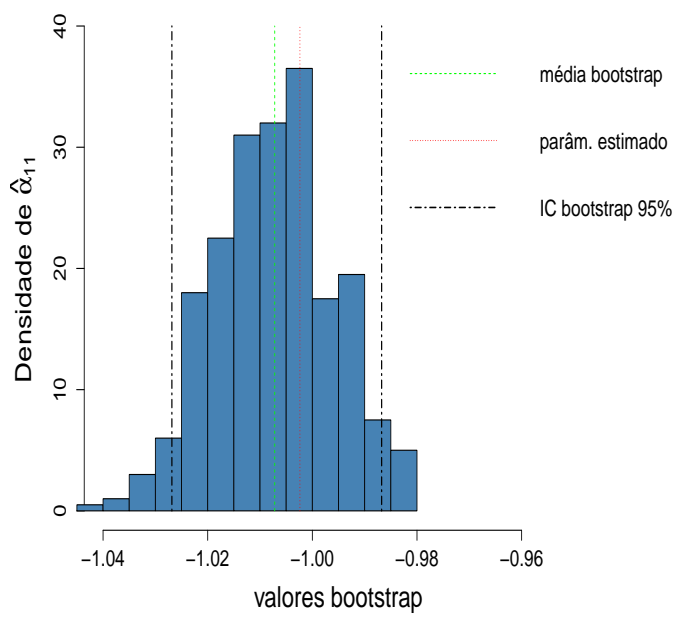

(b)

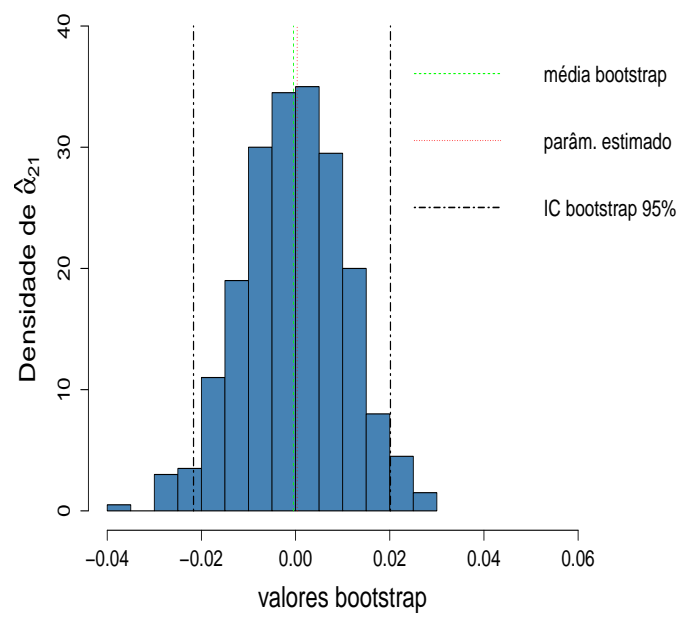

Figura 5.39: Sexto exemplo: Histograma, densidade e quantis bootstrap 2,5\% e 97,5\% sob HA (decrescimento logarítmico) para as componentes $\hat{\alpha}_{11}^{*}(\mathbf{a})$ e $\hat{\alpha}_{21}^{*}(\mathbf{b}) \operatorname{de} \hat{\boldsymbol{\alpha}}^{*}=\left[\begin{array}{ll}\hat{\alpha}_{11}^{*} & \hat{\alpha}_{21}^{*}\end{array}\right]^{\prime}$. 
(a)

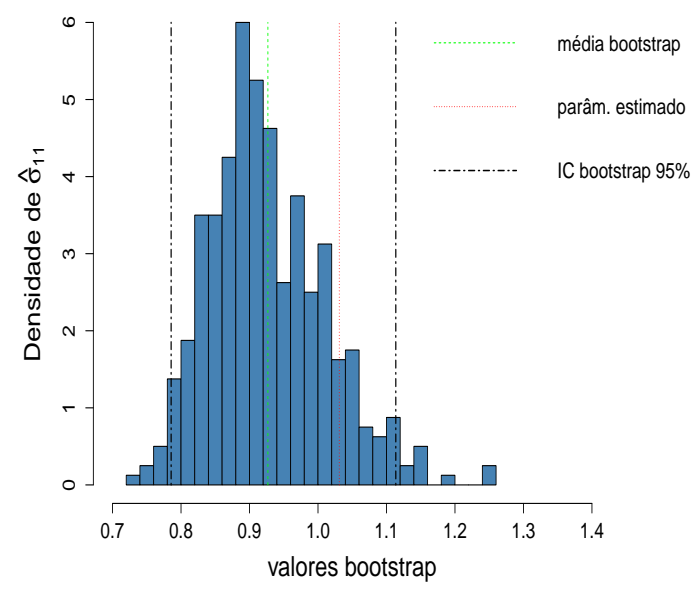

(b)

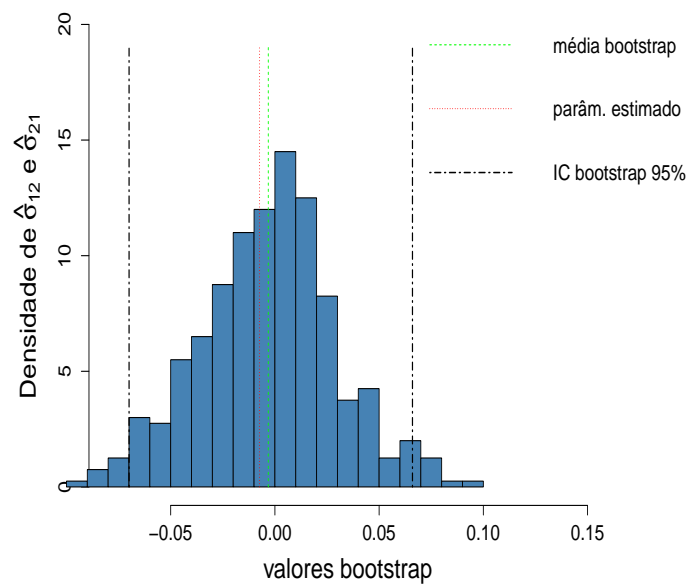

(c)

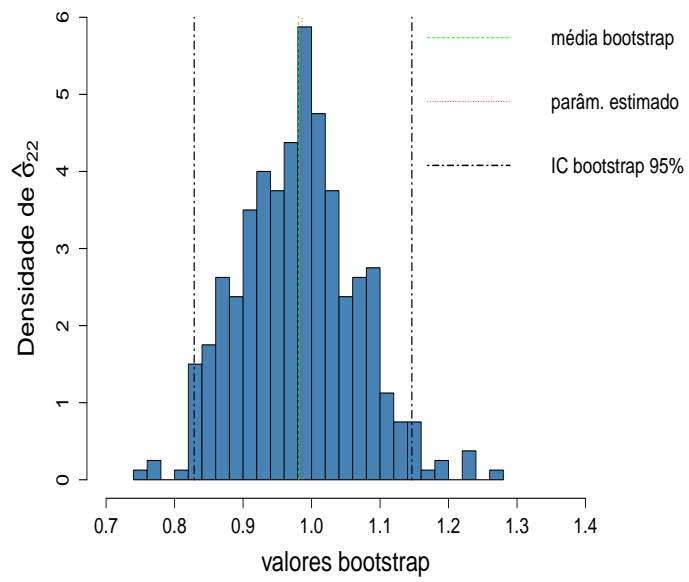

Figura 5.40: Sexto exemplo: Histograma, densidade e quantis bootstrap 2,5\% e 97,5\% sob HA (decrescimento logarítmico) para a componente $\hat{\sigma}_{11}^{*}$ (a), componentes $\hat{\sigma}_{12}^{*}$ e $\hat{\sigma}_{21}^{*}$ (b) e componente $\hat{\sigma}_{22}^{*}(\mathbf{c}) \operatorname{de} \hat{\boldsymbol{\Sigma}}^{*}$.

(a)

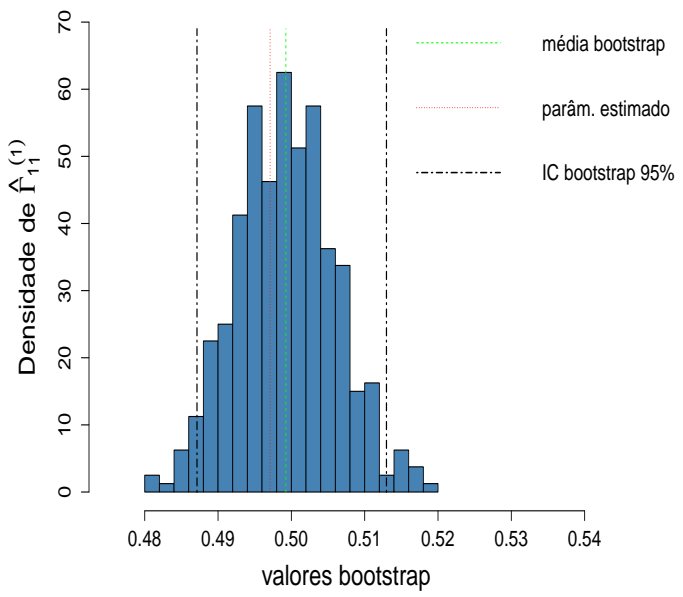

(b)

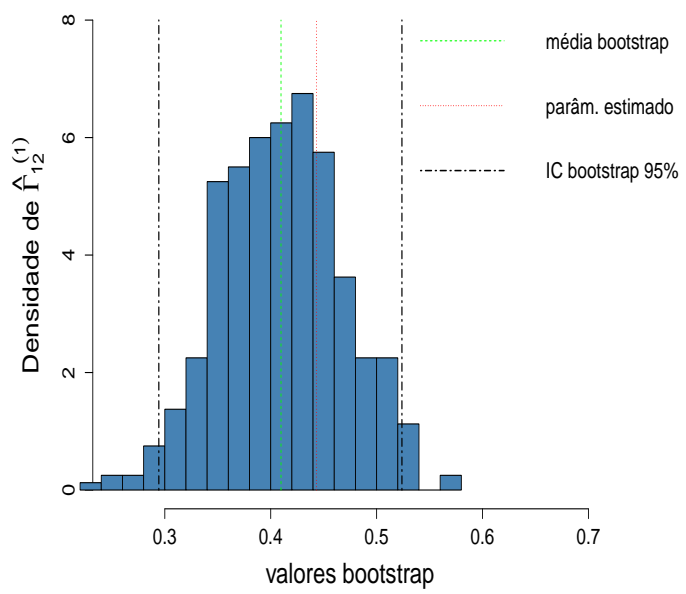

Figura 5.41: Sexto exemplo: Histograma, densidade e quantis bootstrap 2,5\% e 97,5\% sob HA (decrescimento logarítmico)para as componentes $\hat{\Gamma}_{11}^{*(1)}$ (a) e $\hat{\Gamma}_{12}^{*(1)}$ (b) de $\hat{\Gamma}_{\mathbf{1}}^{*}$. 
(c)

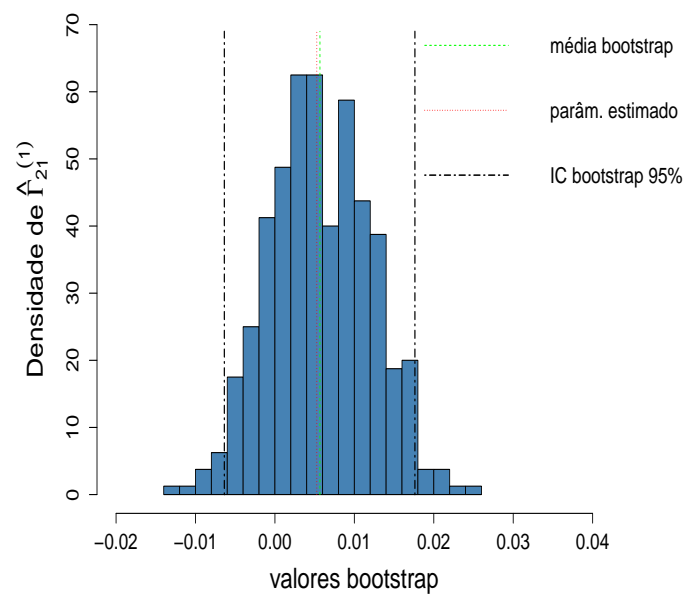

(d)

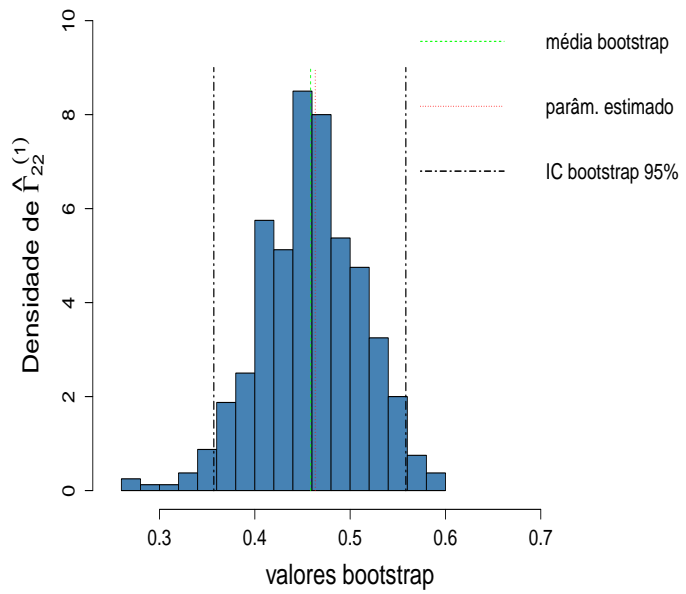

Figura 5.41: Sexto exemplo (continuação): Histograma, densidade e quantis bootstrap 2,5\% e $97,5 \%$ sob HA (decrescimento logarítmico)para as componentes $\hat{\Gamma}_{21}^{*(1)}$ (c) e $\hat{\Gamma}_{22}^{*(1)}$ (d) de $\hat{\Gamma}_{\mathbf{1}}^{*}$.

Apresentamos na Tabela 5.20 estimativas dos parâmetros considerados no modelo, bem como medidas bootstrap de interesse. A Tabela B.2 da Seção B.2 anexa apresenta os demais parâmetros estimados. Podemos notar que a maioria das estimativas e as médias bootstrap estão próximas dos valores teóricos dos parâmetros, que por sua vez estão dentro dos intervalos de confiança bootstrap. As excessões são os parâmetros $\Gamma_{21}^{*(1)}, c_{00}^{*(1,1)}, d_{00}^{*(1,1)}$, $d_{10}^{*(2,1)}, d_{11}^{*(2,1)}, d_{20}^{*(2,1)}, d_{23}^{*(2,1)}, d_{30}^{*(2,1)}, d_{37}^{*(2,1)}, d_{40}^{*(2,1)}, d_{415}^{*(2,1)}, d_{50}^{*(2,1)}$ e $d_{531}^{*(2,1)}$.

Tabela 5.20: Ex.06: Estimativas e medidas bootstrap* sob HA (decrescimento linear)

\begin{tabular}{c|ccccc}
\hline Parâmetro & Teórico & Estimativa & Intervalo de confiança & Média $^{*}$ & Erro Padrão $^{*}$ \\
\hline$R V_{C V T}^{(J)}(\mathrm{HA})$ & - & 2210,08 & {$[1854,71 ; 2502,79]$} & 2230,55 & 171,45 \\
\hline$\alpha_{11}^{*}$ & $-1,0000$ & $-1,0024$ & {$[-1,0268 ;-0,9868]$} & $-1,0072$ & 0,0112 \\
$\alpha_{21}^{*}$ & 0,0000 & 0,0003 & {$[-0,0217 ; 0,0201]$} & $-0,0004$ & 0,0108 \\
\hline$\sigma_{11}^{*}$ & 1,0000 & 1,0310 & {$[0,7856 ; 1,1136]$} & 0,9268 & 0,0855 \\
$\sigma_{12}^{*}=\sigma_{21}^{*}$ & 0,0000 & $-0,0073$ & {$[-0,0700 ; 0,0660]$} & $-0,0032$ & 0,0326 \\
$\sigma_{22}^{*}$ & 1,0000 & 0,9860 & {$[0,8286 ; 1,1464]$} & 0,9808 & 0,0839 \\
\hline$\Gamma_{11}^{*(1)}$ & 0,5000 & 0,4971 & {$[0,4871 ; 0,5130]$} & 0,4993 & 0,0067 \\
$\Gamma_{12}^{*(1)}$ & 0,5000 & 0,0053 & {$[0,2944 ; 0,5239]$} & 0,4097 & 0,0581 \\
$\Gamma_{21}^{* 1}$ & 0,5000 & 0,4432 & {$[-0,0064 ; 0,0176]$} & 0,0057 & 0,0064 \\
$\Gamma_{22}^{*(1)}$ & 0,5000 & 0,4633 & {$[0,3570 ; 0,5584]$} & 0,4586 & 0,0533 \\
\hline$c_{00}^{*(1,1)}$ & 1,0000 & 1,0000 & {$[1,0000 ; 1,0000]$} & 1,0000 & 0,0000 \\
$c_{00}^{*(2,1)}$ & $-4,1142$ & $-4,1265$ & {$[-4,1381 ;-4,1181]$} & $-4,1274$ & 0,0048 \\
\hline$d_{00}^{*(2,1)}$ & 0,0000 & 0,0000 & {$[0,0000 ; 0,0000]$} & 0,0000 & 0,0000 \\
$d_{00}^{*(2,2)}$ & 0,0576 & 0,0435 & {$[0,0321 ; 0,0518]$} & 0,0420 & 0,0047 \\
\hline$d_{10}^{*(2,1)}$ & 0,0000 & 0,0000 & {$[0,0000 ; 0,0000]$} & 0,0000 & 0,0000 \\
\hline
\end{tabular}

(Continua na página seguinte) 
Tabela 5.20 - Continuação da página anterior

\begin{tabular}{|c|c|c|c|c|c|}
\hline Parâmetro & Teórico & Estimativa & Intervalo de confiança* & Média* & Erro Padrão* \\
\hline$d_{10}^{*(2,2)}$ & $-0,1505$ & $-0,1652$ & {$[-0,1766 ;-0,1567]$} & $-0,1663$ & 0,0051 \\
\hline$d_{11}^{*(2,1)}$ & 0,0000 & 0,0000 & {$[0,0000 ; 0,0000]$} & 0,0000 & 0,0000 \\
\hline$d_{11}^{*(2,1)}$ & 0,1716 & 0,1577 & {$[0,1478 ; 0,1651]$} & 0,1567 & 0,0041 \\
\hline$d_{20}^{*(1,1)}$ & 0,0000 & 0,0000 & {$[0,0000 ; 0,0000]$} & 0,0000 & 0,0000 \\
\hline$d_{20}^{*(2,1)}$ & $-0,1025$ & $-0,1214$ & {$[-0,1325 ;-0,1118]$} & $-0,1224$ & 0,0050 \\
\hline$d_{21}^{*(1,1)}$ & 0,0000 & 0,0000 & {$[0,0000 ; 0,0000]$} & 0,0000 & 0,0000 \\
\hline$d_{21}^{*(2,1)}$ & 0,0285 & 0,0270 & {$[0,0161 ; 0,0375]$} & 0,0273 & 0,0050 \\
\hline$d_{22}^{*(1,1)}$ & 0,0000 & 0,0000 & {$[0,0000 ; 0,0000]$} & 0,0000 & 0,0000 \\
\hline$d_{22}^{*(2,1)}$ & $-0,0191$ & $-0,0227$ & {$[-0,0323 ;-0,0129]$} & $-0,0228$ & 0,0040 \\
\hline$d_{23}^{*(1,1)}$ & 0,0000 & 0,0000 & {$[0,0000 ; 0,0000]$} & 0,0000 & 0,0000 \\
\hline$d_{23}^{*(2,1)}$ & 0,0910 & 0,0702 & {$[0,0596 ; 0,0805$} & 0,0690 & 0,0052 \\
\hline$d_{30}^{*(1,1)}$ & 0,0000 & 0,0000 & {$[0,0000 ; 0,0000]$} & 0,0000 & 0,0000 \\
\hline$d_{30}^{*(2,1)}$ & $-0,0831$ & $-0,0952$ & {$[-0,1069 ;-0,0836]$} & $-0,0956$ & 0,0054 \\
\hline$d_{31}^{*(1,1)}$ & 0,0000 & 0,0000 & {$[0,0000 ; 0,0000]$} & 0,0000 & 0,0000 \\
\hline$d_{31}^{*(2,1)}$ & 0,0179 & 0,0201 & {$[0,0075 ; 0,0288]$} & 0,0199 & 0,0049 \\
\hline$d_{32}^{*(1,1)}$ & 0,0000 & 0,0000 & {$[0,0000 ; 0,0000]$} & 0,0000 & 0,0000 \\
\hline$d_{32}^{*(2,1)}$ & $-0,0046$ & $-0,0001$ & {$[-0,0124 ; 0,0080]$} & $-0,0004$ & 0,0048 \\
\hline$d_{33}^{*(1,1)}$ & 0,0000 & 0,0000 & {$[0,0000 ; 0,0000]$} & 0,0000 & 0,0000 \\
\hline$d_{33}^{*(2,1)}$ & 0,0005 & 0,0022 & {$[-0,0093 ; 0,0107]$} & 0,0020 & 0,0045 \\
\hline$d_{34}^{*(1,1)}$ & 0,0000 & 0,0000 & {$[0,0000 ; 0,0000]$} & 0,0000 & 0,0000 \\
\hline$d_{34}^{*(2,1)}$ & $-0,0008$ & $-0,0045$ & {$[-0,0133 ; 0,0046]$} & $-0,0045$ & 0,0039 \\
\hline$d_{35}^{*(1,1)}$ & 0,0000 & 0,0000 & {$[0,0000 ; 0,0000]$} & 0,0000 & 0,0000 \\
\hline$d_{35}^{*(2,1)}$ & 0,0049 & 0,0061 & {$[-0,0051 ; 0,0130]$} & 0,0057 & 0,0038 \\
\hline$d_{36}^{*(1,1)}$ & 0,0000 & 0,0000 & {$[0,0000 ; 0,0000]$} & 0,0000 & 0,0000 \\
\hline$d_{36}^{*(2,1)}$ & $-0,0093$ & $-0,0090$ & {$[-0,0171 ; 0,0009]$} & $-0,0089$ & 0,0041 \\
\hline$d_{37}^{*(1,1)}$ & 0,0000 & 0,0000 & {$[0,0000 ; 0,0000]$} & 0,0000 & 0,0000 \\
\hline$d_{37}^{*(2,1)}$ & 0,0541 & 0,0241 & {$[0,0130 ; 0,0335]$} & 0,0221 & 0,0050 \\
\hline$\vdots$ & $\vdots$ & $\vdots$ & $\vdots$ & $\vdots$ & $\vdots$ \\
\hline$d_{531}^{(1,1)}$ & 0,0000 & 0,0000 & {$\left[\begin{array}{lll}0,0000 & 0,0000\end{array}\right]$} & 0,0000 & 0,0000 \\
\hline$d_{531}^{(2,1)}$ & $-0,0025$ & $-0,0074$ & {$[-0,0145-0,0036$} & $-0,0082$ & 0,0027 \\
\hline
\end{tabular}




\section{Capítulo 6}

\section{Aplicação a dados reais}

Temos o interesse em investigar a hipótese da Paridade de Poder de Compra (PPC) entre diferentes países. De acordo com Falk e Wang (2003), esta é uma das hipóteses mais detalhadamente examinadas no contexto de finanças internacionais ou até mesmo na própria Economia. Conforme salienta Johansen (1995), é possível encontrar relações de cointegração ao formular hipóteses econômicas simples em termos de parâmetros.

Falk e Wang (2003) investigaram a validade da hipótese de PPC entre índices de preços dos Estados Unidos (tomado como país local) e outros doze países (tomados como países estrangeiros), a saber: Bélgica, Canadá, Dinamarca, França, Alemanha, Itália, Japão, Holanda, Noruega, Espanha, Suécia e Reino Unido. No trabalho, os autores fizeram a suposição de que os índices de preços seguem uma distribuição estável com variância infinita. Em suas análises, foi utilizado um conjunto de dados extraído do International Financial Statistics - IFS (IMF, 2016), que também pode ser acessado na página da revista em que o artigo foi publicado. Este conjunto de dados possui frequência mensal e foi amostrado de janeiro de 1973 a dezembro de 1999, resultando em 324 observações.

Uma condição necessária para que haja cointegração é que as séries componentes sejam integradas de ordem um, I(1). Falk e Wang (2003) fizeram testes de raiz unitária de Phillips-Perron (Phillips e Perron, 1988) e concluiram que não é possível rejeitar a hipótese de raiz unitária para os índices de preços e para as taxas de câmbio destes países. Além disso, os autores rejeitaram a hipótese de raiz unitária para a primeira diferença das séries. Estes resultados foram obtidos tanto com a suposição de distribuições estáveis quanto a suposição de distribuições normais para os índices de preços e taxas de câmbio. Foram encontradas ainda evidências de cointegração usual (ou invariante com o tempo) entre Estados Unidos e os demais países, exceto para Canadá e Alemanha.

Conforme apontam Bierens e Martins (2010), a presença de fatores, como o custo de transação por exemplo, são razões pelas quais os modelos VEC lineares podem ser incapazes de detectar PPC de longo prazo. Segundo os autores, a presença de tais fatores leva a processos com ajuste não linear na relação de PPC.

Com base nestas idéias, Martins (2005) e Bierens e Martins (2010) consideraram a 
PPC sob uma nova perspectiva. Motivados pelo trabalho de Park e Hahn (1999), os autores testaram a hipótese nula de cointegração usual, confome proposto por Engle e Granger (1987) e Johansen (1995), contra a hipótese de coitegração variando com o tempo. Para isso, eles modelaram o vetor de cointegração temporalmente por meio de polinômios de Chebyshev. Em suas análises, eles utilizaram o mesmo conjunto de dados empregado em Falk e Wang (2003). Conforme justificam Bierens e Martins (2010), trabalhar com um conjunto de dados previamente utilizado em outros contextos garante a relevância empírica das observações. Os autores encontraram evidências de cointegração variando com o tempo entre Estados Unidos e os demais doze países. Os resultados encontrados refutam os achados de Falk e Wang (2003) para Canadá e Alemanha, que afirmam não haver relação de PPC com os Estados Unidos.

Em um trabalho recente, Martins (2016) apresentou um estudo via simulações de Monte Carlo e Bootstrap para o modelo de cointegração variando com o tempo apresentado em Bierens e Martins (2010). O autor aborda novamente o caso da PPC entre Estados Unidos e os doze países citados anteriormente. Refutando os próprios achados em Bierens e Martins (2010), Martins (2016) indica que existem evidências de que a PPC entre Estados Unidos e o Reino Unido parece ser invariante ao longo do tempo. O artigo é interessante no sentido de trazer melhores resultados para casos com pequenas amostras, que apresentam distorção quanto ao tamanho do teste no caso assintótico (Bierens e Martins, 2010).

Neste trabalho, deseja-se utilizar o mesmo conjunto de dados empregado em Falk e Wang (2003), Bierens e Martins (2010) e em Martins (2016) para testar a hipótese de PPC variando com o tempo. O vetor de cointegração no entanto, será modelado temporalmente por meio de ondaletas, conforme conforme o modelo definido no Capítulo 4 . O intuito foi testar a hipótes de PPC entre Estados Unidos e os demais doze países sob esta nova perspectiva de modelagem para o vetor de cointegração. Em contrapartida, foram comparados os resultados obtidos com os resultados apresentados em Bierens e Martins (2010) e Martins (2016). De acordo com com estes dois trabalhos, temos o interesse em fazer oposição à versão padrão da PPC utilizada em Falk e Wang (2003).

Na Figura 6.1 são apresentados os índices de preços dos países citados anteriomente, juntamente com a taxa de câmbio em dólares americanos. Em todos os casos, os Estados Unidos foram considerados o país local e os demais países considerados o país estrangeiro. As séries são apresentadas em base logarítmica (natural). 

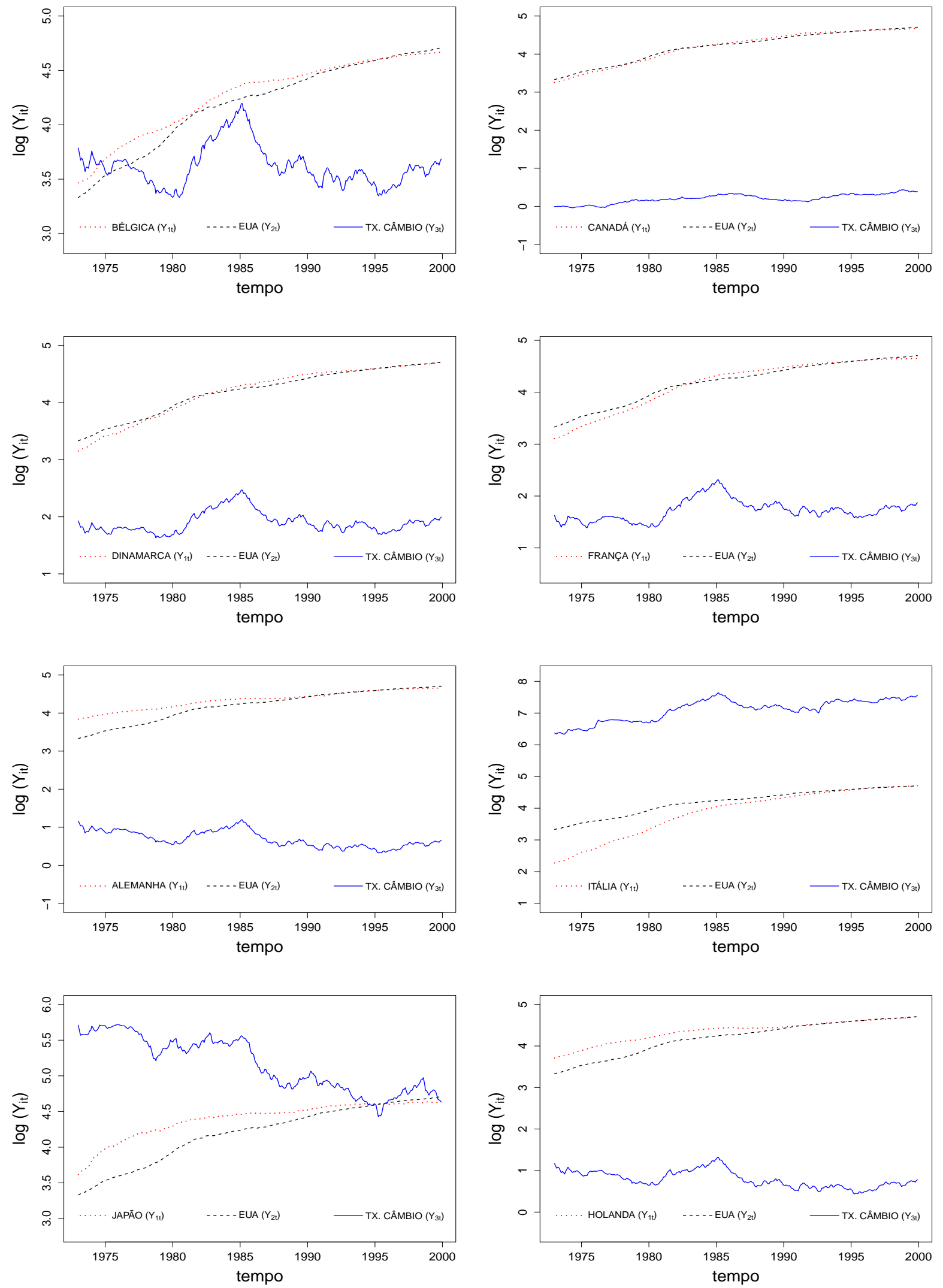

Figura 6.1: Gráficos com índices de preços dos EUA (país local) e dos países estrangeiros Bélgica, Canadá, Dinamarca, França, Alemanha, Itália, Japão e Holanda, juntamente com as respectivas taxas de câmbio. 

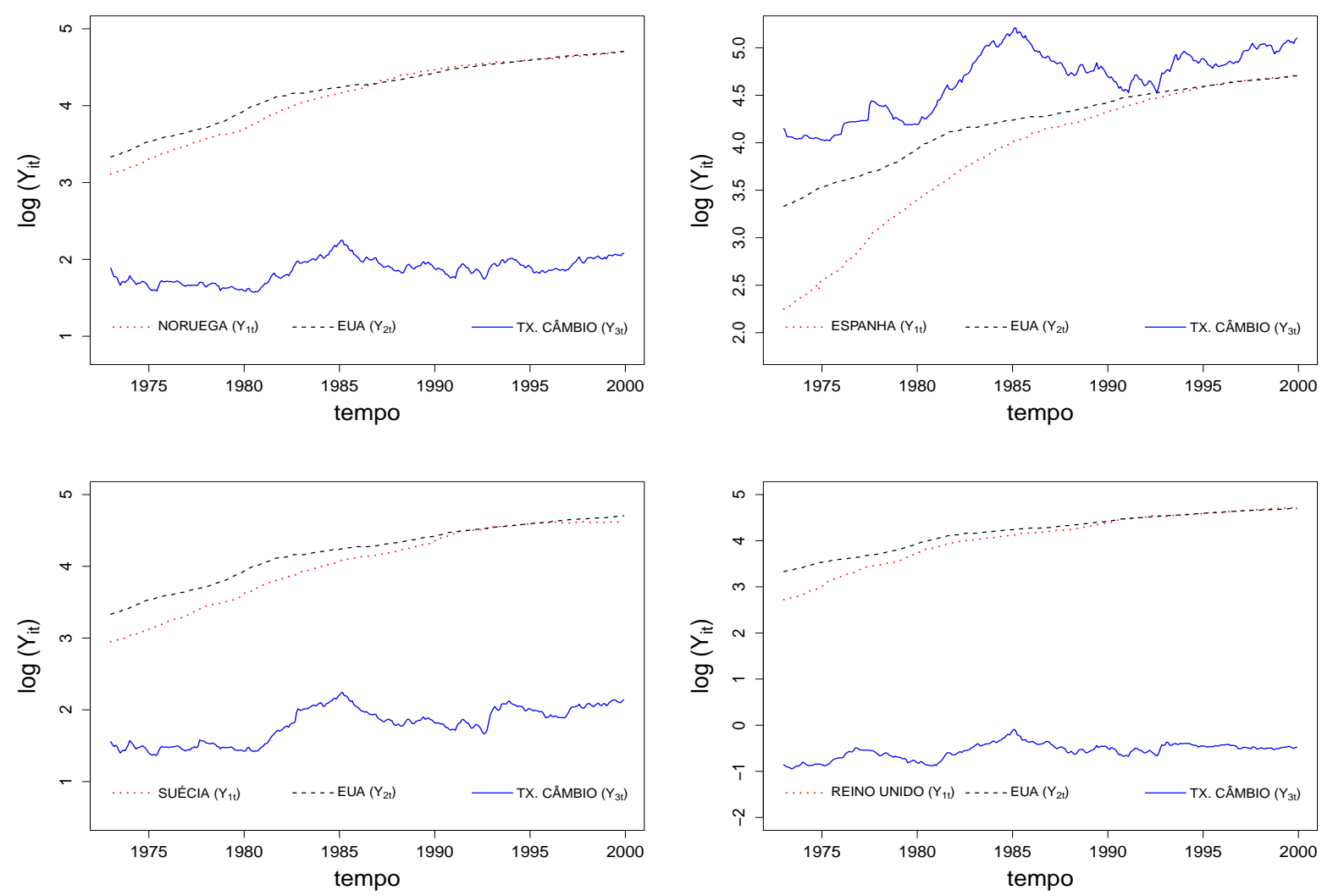

Figura 6.1: (continuação) Gráficos com índices de preços dos EUA (país local) e dos países estrangeiros Noruega, Espanha, Suécia e Reino Unido, juntamente com as respectivas taxas de câmbio.

\subsection{Paridade de Poder de Compra com coeficientes variando com o tempo - caso sem intercepto}

Seguindo a notação de Falk e Wang (2003), Bierens e Martins (2010) e Martins (2016), temos que

$$
Y_{t}=\left[\begin{array}{l}
Y_{1 t} \\
Y_{2 t} \\
Y_{3 t}
\end{array}\right]=\left[\begin{array}{c}
\ln S_{t} \\
\ln P_{t} \\
\ln P_{t}^{*}
\end{array}\right]
$$

onde $S_{t}$ representa a taxa de câmbio (expresso em moeda do país local por unidade do país estrangeiro), $P_{t}$ representa o índice de preços do país local (Estados Unidos) e $P_{t}^{*}$ representa o índice de preços do país estrangeiro (demais países considerados). Além disso, $\ln ($.$) representa o logarítmo natural.$

Assim como apresentado em Bierens e Martins (2010) e Martins (2016), foi testada a hipótese de cointegração variando com o tempo versus cointegração usual, entre Estados Unidos e os demais países. Para isso, conforme apresentado no Capítulo 4, foi empregado o modelo cointegrado na forma de correção de erros e vetor de cointegração variando com o tempo, decomposto temporalmente por meio de ondaletas, definido a seguir: 


$$
\left[\begin{array}{l}
\Delta Y_{1 t} \\
\Delta Y_{2 t} \\
\Delta Y_{3 t}
\end{array}\right]=\left[\begin{array}{l}
\alpha_{1} \\
\alpha_{2} \\
\alpha_{3}
\end{array}\right]\left[\begin{array}{lll}
\beta_{1 t} & \beta_{2 t} & \beta_{3 t}
\end{array}\right]\left[\begin{array}{l}
Y_{1, t-1} \\
Y_{2, t-1} \\
Y_{3, t-1}
\end{array}\right]+\boldsymbol{\Gamma} \Delta X+\left[\begin{array}{l}
u_{1 t} \\
u_{2 t} \\
u_{3 t}
\end{array}\right]
$$

onde $\Gamma$ e $\Delta X$ são definidos na forma matricial em 4.10 e 4.11 . Note que se não forem incluídas defasagens no modelo, o termo $\Gamma \Delta X$ é retirado de 6.2 .

Em suas análises, Bierens e Martins (2010) e Martins (2016) consideraram apenas as defasagens $p=1,6,10$ e 18 como possíveis escolhas para lidar com a autocorrelação presente nas séries de resíduos para os modelos ajustados. Martins (2016) utiliza o critério HQ (Hannan e Quinn, 1979) para escolher entre as defasagens, tendo decidido por $p=1$.

Neste trabalho, utilizando ondaletas para a decomposição do vetor de cointegração, foi possível notar a necessidade de incluir defasagens maiores que $p=1$ para excluir a autocorrelação presente nas séries de resíduos dos modelos ajustados. No entanto, para comparar os resultados obtidos com aqueles apresentados em Bierens e Martins (2010) e Martins (2016), foi utilizada inicialmente a defasagem $p=1$. Ajustes com defasagens maiores serão considerados posteriormente.

A Tabela 6.1 contém as estimativas do modelo e medidas bootstrap $\left(^{\star}\right)$ obtidas para a estatística $R V_{C V T}^{(J)}$ de razão de verossimilhanças, dada em 4.81, para testar a hipótese nula de cointegração constante ao longo do tempo versus cointegração variando com tempo. Foi considerada a família de ondaletas Daubechies D2, com $p=1$ e $J=0,1,2,3$ e 4 . Cada linha da tabela foi gerada com base em $B=399$ simulações bootstrap (método wild, Seção 4.6). Esta é a mesma quantidade de simulações utilizada em Martins (2016).

Tabela 6.1: Estatística $R V_{C V T}^{(J)}$ e medidas bootstrap $\left(^{\star}\right)$ para testar a PPC constante ao longo do tempo versus $\mathrm{PPC}$ variando com o tempo.

\begin{tabular}{c|c|c|c|c|c|c|c|c}
\hline País estrangeiro & $\mathrm{p}$ & $\mathrm{J}$ & N.C.O. $^{\dagger}$ & $R V_{C V T}^{(J)}$ & I.C. $^{\star}$ & Média $^{\star}$ & Erro Padrão $^{\star}$ & ${\text { Valor- } p^{\star}}^{\text {Bélgica }}$ \\
& 1 & 0 & 2 & 89,50 & {$[0,15 ; 9,83]$} & 3,28 & 2,62 & 0,0000 \\
& 1 & 1 & 4 & 142,27 & {$[2,64 ; 20,52]$} & 9,67 & 4,64 & 0,0000 \\
& 1 & 2 & 8 & 195,88 & {$[12,15 ; 40,76]$} & 24,40 & 7,31 & 0,0000 \\
& 1 & 3 & 16 & 279,29 & {$[34,11 ; 89,33]$} & 56,29 & 13,36 & 0,0000 \\
& 1 & 4 & 32 & 403,58 & {$[92,75 ; 181,79]$} & 134,50 & 23,14 & 0,0000 \\
\hline Canadá & 1 & 0 & 2 & 72,35 & {$[0,29 ; 10,78]$} & 3,30 & 2,62 & 0,0000 \\
& 1 & 1 & 4 & 84,04 & {$[2,50 ; 20,61]$} & 9,97 & 4,52 & 0,0000 \\
& 1 & 2 & 8 & 137,80 & {$[12,27 ; 45,53]$} & 25,66 & 8,74 & 0,0000 \\
& 1 & 3 & 16 & 176,54 & {$[36,95 ; 93,73]$} & 60,48 & 14,31 & 0,0000 \\
& 1 & 4 & 32 & 284,96 & {$[101,86 ; 192,96]$} & 144,14 & 23,60 & 0,0000 \\
\hline Dinamarca & 1 & 0 & 2 & 136,92 & {$[0,22 ; 9,09]$} & 3,09 & 2,44 & 0,0000 \\
& 1 & 1 & 4 & 171,36 & {$[2,58 ; 22,69]$} & 9,91 & 5,18 & 0,0000 \\
& 1 & 2 & 8 & 193,27 & {$[12,59 ; 41,60]$} & 24,47 & 7,36 & 0,0000 \\
& 1 & 3 & 16 & 252,79 & {$[33,73 ; 89,22]$} & 58,42 & 13,68 & 0,0000 \\
& 1 & 4 & 32 & 353,21 & {$[96,08 ; 191,44]$} & 139,93 & 22,74 & 0,0000 \\
\hline França & 1 & 0 & 2 & 171,30 & {$[0,23 ; 9,54]$} & 3,24 & 2,65 & 0,0000 \\
\hline
\end{tabular}

(Continua na página seguinte) 
Tabela 6.1 - Continuação da página anterior

\begin{tabular}{|c|c|c|c|c|c|c|c|c|}
\hline País estrangeiro & $\mathrm{p}$ & $\mathrm{J}$ & N.C.O. ${ }^{\dagger}$ & $R V_{C V T}^{(J)}$ & I.C. ${ }^{*}$ & Média* & Erro Padrão* & Valor- $p^{*}$ \\
\hline & 1 & 1 & 4 & 201,29 & {$[2,46 ; 18,88]$} & 9,33 & 4,40 & 0,0000 \\
\hline & 1 & 2 & 8 & 226,94 & {$[10,97 ; 40,19]$} & 23,87 & 7,68 & 0,0000 \\
\hline & 1 & 3 & 16 & 289,59 & {$[35,07 ; 81,89]$} & 56,98 & 11,91 & 0,0000 \\
\hline & 1 & 4 & 32 & 399,15 & [ 101,10;188,96 ] & 140,39 & 21,82 & 0,0000 \\
\hline \multirow[t]{5}{*}{ Alemanha } & 1 & 0 & 2 & 13,26 & {$[0,24 ; 11,12]$} & 3,41 & 2,85 & 0,0125 \\
\hline & 1 & 1 & 4 & 49,54 & {$[2,90 ; 21,72]$} & 10,08 & 4,83 & 0,0000 \\
\hline & 1 & 2 & 8 & 83,37 & {$[12,14 ; 44,71]$} & 25,39 & 8,37 & 0,0000 \\
\hline & 1 & 3 & 16 & 133,42 & {$[34,63 ; 90,97]$} & 59,69 & 14,56 & 0,0000 \\
\hline & 1 & 4 & 32 & 254,08 & {$[100,23 ; 195,22]$} & 142,05 & 25,26 & 0,0025 \\
\hline \multirow[t]{5}{*}{ Itália } & 1 & 0 & 2 & 13,19 & {$[0,20 ; 9,69]$} & 2,98 & 2,40 & 0,0025 \\
\hline & 1 & 1 & 4 & 26,61 & {$[2,44 ; 19,72]$} & 9,25 & 4,67 & 0,0025 \\
\hline & 1 & 2 & 8 & 48,19 & {$[11,07 ; 41,13]$} & 23,73 & 7,45 & 0,0025 \\
\hline & 1 & 3 & 16 & 74,31 & {$[35,93 ; 91,65]$} & 59,95 & 13,52 & 0,1328 \\
\hline & 1 & 4 & 32 & 209,34 & {$[102,31 ; 196,70$ ] } & 146,36 & 24,19 & 0,0125 \\
\hline \multirow[t]{5}{*}{ Japão } & 1 & 0 & 2 & 45,13 & {$[0,18 ; 8,95]$} & 3,08 & 2,38 & 0,0000 \\
\hline & 1 & 1 & 4 & 88,77 & {$[3,05 ; 18,66]$} & 9,48 & 4,69 & 0,0000 \\
\hline & 1 & 2 & 8 & 115,39 & {$[11,44 ; 45,04]$} & 24,53 & 8,48 & 0,0000 \\
\hline & 1 & 3 & 16 & 156,57 & {$[33,98 ; 85,00]$} & 56,61 & 12,95 & 0,0000 \\
\hline & 1 & 4 & 32 & 283,47 & {$[93,93 ; 184,79]$} & 132,88 & 24,39 & 0,0000 \\
\hline \multirow[t]{5}{*}{ Holanda } & 1 & 0 & 2 & 10,81 & {$[0,22 ; 9,83]$} & 3,10 & 2,56 & 0,0150 \\
\hline & 1 & 1 & 4 & 58,86 & {$[3,02 ; 20,87]$} & 9,54 & 4,50 & 0,0000 \\
\hline & 1 & 2 & 8 & 87,78 & {$[11,07 ; 40,50]$} & 23,87 & 7,42 & 0,0000 \\
\hline & 1 & 3 & 16 & 134,42 & {$[34,68 ; 91,13]$} & 57,84 & 14,08 & 0,0000 \\
\hline & 1 & 4 & 32 & 249,86 & {$[95,20 ; 191,40]$} & 140,60 & 23,95 & 0,0000 \\
\hline \multirow[t]{5}{*}{ Noruega } & 1 & 0 & 2 & 75,09 & {$[0,12 ; 10,12]$} & 3,16 & 2,55 & 0,0000 \\
\hline & 1 & 1 & 4 & 81,30 & {$[2,94 ; 22,03]$} & 9,73 & 4,72 & 0,0000 \\
\hline & 1 & 2 & 8 & 117,99 & {$[11,12 ; 44,89]$} & 24,39 & 8,39 & 0,0000 \\
\hline & 1 & 3 & 16 & 172,37 & {$[36,87 ; 81,53]$} & 56,54 & 12,33 & 0,0000 \\
\hline & 1 & 4 & 32 & 265,02 & {$[93,95 ; 192,49$ ] } & 139,39 & 23,83 & 0,0000 \\
\hline \multirow[t]{5}{*}{ Espanha } & 1 & 0 & 2 & 2,04 & {$[0,16 ; 10,52]$} & 3,04 & 2,65 & 0,5439 \\
\hline & 1 & 1 & 4 & 25,24 & {$[2,97 ; 20,78]$} & 9,51 & 4,51 & 0,0075 \\
\hline & 1 & 2 & 8 & 37,08 & {$[11,53 ; 41,12]$} & 23,63 & 7,75 & 0,0627 \\
\hline & 1 & 3 & 16 & 91,41 & {$[34,41 ; 81,72]$} & 55,15 & 12,24 & 0,0000 \\
\hline & 1 & 4 & 32 & 190,48 & {$[96,48 ; 189,92]$} & 136,71 & 22,03 & 0,0175 \\
\hline \multirow[t]{5}{*}{ Suécia } & 1 & 0 & 2 & 9,34 & {$[0,29 ; 9,29]$} & 3,13 & 2,39 & 0,0276 \\
\hline & 1 & 1 & 4 & 35,68 & {$[2,67 ; 20,97]$} & 9,69 & 4,64 & 0,0000 \\
\hline & 1 & 2 & 8 & 58,86 & {$[10,12 ; 42,07]$} & 23,56 & 8,29 & 0,0025 \\
\hline & 1 & 3 & 16 & 122,03 & {$[35,13 ; 80,63]$} & 55,53 & 12,10 & 0,0000 \\
\hline & 1 & 4 & 32 & 237,83 & {$[90,74 ; 177,51]$} & 129,54 & 20,77 & 0,0000 \\
\hline \multirow[t]{5}{*}{ Reino Unido } & 1 & 0 & 2 & 8,75 & {$[0,12 ; 8,49]$} & 2,86 & 2,29 & 0,0175 \\
\hline & 1 & 1 & 4 & 47,84 & {$[2,32 ; 21,59]$} & 9,26 & 4,66 & 0,0000 \\
\hline & 1 & 2 & 8 & 96,01 & {$[10,52 ; 41,42]$} & 23,93 & 8,30 & 0,0000 \\
\hline & 1 & 3 & 16 & 176,01 & {$[33,54 ; 85,13]$} & 57,50 & 12,54 & 0,0000 \\
\hline & 1 & 4 & 32 & 287,03 & {$[96,20 ; 187,27]$} & 138,14 & 21,80 & 0,0000 \\
\hline
\end{tabular}

( Número de coeficientes de ondaletas: N.C.O. $=2^{J+1}$.) 
Com base nos resultados obtidos na Tabela 6.1 e tomando um nível de significância de $5 \%$, é possível afirmar que existem fortes evidências em favor de cointegração variando com o tempo entre Estados Unidos e os países: Bélgica, Canadá, Dinamarca, França, Alemanha, Japão, Holanda, Noruega, Suécia e Reino Unido. Note que em todos estes casos, foram obtidos valores-p ${ }^{\star}$ muito próximos de zero, para todos os valores de $J$ considerados. Este achados estão em concordância com os obtidos em Bierens e Martins (2010) e Martins (2016), que também encontraram evidência de cointegração variando com o tempo para estes países. A única resssalva é sobre os resultados obtidos para a relação de cointegração entre Estados Unidos e Reino Unido. Conforme citado anteriormente, Martins (2016) encontrou evidências de cointegração constante entre os dois países, refutando os próprios resultados em Bierens e Martins (2010). Os resultados obtidos na Tabela 6.1 para a relação de cointegração entre Estados Unidos e Reino Unido estão em concordância com os resultados obtidos em Bierens e Martins (2010), que também encontraram evidências de cointegração variando com o tempo para os dois países.

É possível notar ainda pela Tabela 6.1, que existem evidências que nos levam a não rejeitar a hipótese nula de cointegração variando com tempo entre Estados Unidos e os países Itália e Espanha para alguns valores de $J$. Dependendo da aproximação proposta para $\boldsymbol{\beta}_{t}$, parece haver uma relação de cointegração constante entre estes países. Estes achados diferem daqueles apresentados em Bierens e Martins (2010) e Martins (2016).

Uma vez que os modelos foram estimados, conforme resultados apresentados na Tabela 6.1, temos condições de construir os resíduos de cointegração estimados, dados por $\hat{e}_{t}=\hat{\boldsymbol{\beta}}_{t}^{\prime} Y_{t}$, com $\boldsymbol{\beta}_{t}=\left[\begin{array}{lll}\hat{\beta}_{1 t} & \hat{\beta}_{2 t} & \hat{\beta}_{3 t}\end{array}\right]^{\prime}$. Seguindo os critérios apresentados em Cheung e Lai (1993), Martins (2016) utiliza as seguintes regras para que haja cointegração entre Estados Unidos e os demais países: $\hat{e}_{t}$ deve ser estacionário, além de $\beta_{2 t}<0$ e $\beta_{3 t}>0$. Apesar de apresentar os critérios para que a cointegração seja verificada, Martins (2016) e Bierens e Martins (2010), limitam-se a comentar sobre os gráficos de $\boldsymbol{\beta}_{t}$, que seguem as regras descritas em Cheung e Lai (1993) para a maior parte dos valores de $t$. No entanto, os trabalhos não fazem qualquer teste para testar a estacionariedade de $\hat{e}_{t}$.

Para testar a estacionariedade dos resíduos de cointegração estimados $\hat{e}_{t}$, deste ponto em diante chamados de RCE, são apresentados na Tabela 6.2 os valores- $p$ dos testes Dickey-Fuller aumentado (ADF) (Said e Dickey, 1984) e de raiz unitária de PhillipsPerron (PP) (Phillips e Perron, 1988) para os RCE's dos modelos estimados na Tabela 6.1. Nos dois testes, a hipótese alternativa $H_{A}$ é de que as séries são estacionárias. Adicionalmente, foi testada também a suposição de normalidade dos resíduos $\hat{u}_{t}$ dos modelos ajustados. Para isso foi empregado o teste de normalidade de Shapiro Wilk (Shapiro e Wilk, 1965), onde os valores- $p$ referem-se à hipótese nula $H_{0}$ de que a série testada segue uma distribuição normal. 
Tabela 6.2: Resultados dos testes de raiz unitária $(H 0)$ e de normalidade $(H 0)$ para os resíduos de cointegração estimados $\hat{e}_{t}=\hat{\boldsymbol{\beta}}_{t}^{\prime} Y_{t}$.

\begin{tabular}{|c|c|c|c|c|c|c|c|c|}
\hline \multicolumn{4}{|c|}{ País local: Estados Unidos } & \multicolumn{2}{|c|}{ Estacionariedade de RCE } & \multicolumn{3}{|c|}{ Teste de Shapiro-Wilk } \\
\hline País estrangeiro & $\mathrm{p}$ & $\mathrm{J}$ & N.C.O. ${ }^{\dagger}$ & Teste ADF & Teste PP & $u_{1 t}$ & $u_{2 t}$ & $u_{3 t}$ \\
\hline \multirow{5}{*}{ Bélgica } & 1 & 0 & 2 & 0,01 & 0,01 & 0,4666 & 0,0005 & 0,0558 \\
\hline & 1 & 1 & 4 & 0,01 & 0,01 & 0,4717 & 0,0015 & 0,0045 \\
\hline & 1 & 2 & 8 & 0,01 & 0,01 & 0,4660 & 0,0043 & 0,0000 \\
\hline & 1 & 3 & 16 & 0,01 & 0,01 & 0,4675 & 0,0003 & 0,0033 \\
\hline & 1 & 4 & 32 & 0,01 & 0,01 & 0,4477 & 0,0007 & 0,0009 \\
\hline \multirow{5}{*}{ Canadá } & 1 & 0 & 2 & 0,13 & 0,44 & 0,7329 & 0,0000 & 0,0014 \\
\hline & 1 & 1 & 4 & 0,01 & 0,01 & 0,7279 & 0,0000 & 0,0002 \\
\hline & 1 & 2 & 8 & 0,01 & 0,01 & 0,7353 & 0,0000 & 0,0003 \\
\hline & 1 & 3 & 16 & 0,01 & 0,01 & 0,7517 & 0,0000 & 0,0004 \\
\hline & 1 & 4 & 32 & 0,01 & 0,01 & 0,8060 & 0,0000 & 0,0066 \\
\hline \multirow[t]{5}{*}{ Dinamarca } & 1 & 0 & 2 & 0,01 & 0,01 & 0,1421 & 0,0000 & 0,0001 \\
\hline & 1 & 1 & 4 & 0,01 & 0,01 & 0,1400 & 0,0000 & 0,0000 \\
\hline & 1 & 2 & 8 & 0,01 & 0,01 & 0,1489 & 0,0000 & 0,0000 \\
\hline & 1 & 3 & 16 & 0,01 & 0,01 & 0,1489 & 0,0000 & 0,0000 \\
\hline & 1 & 4 & 32 & 0,01 & 0,01 & 0,1956 & 0,0000 & 0,0024 \\
\hline \multirow[t]{5}{*}{ França } & 1 & 0 & 2 & 0,01 & 0,01 & 0,2481 & 0,0109 & 0,0000 \\
\hline & 1 & 1 & 4 & 0,01 & 0,01 & 0,2217 & 0,0020 & 0,0000 \\
\hline & 1 & 2 & 8 & 0,01 & 0,01 & 0,2333 & 0,0016 & 0,0001 \\
\hline & 1 & 3 & 16 & 0,01 & 0,01 & 0,2431 & 0,0924 & 0,0000 \\
\hline & 1 & 4 & 32 & 0,01 & 0,01 & 0,2662 & 0,0103 & 0,0000 \\
\hline \multirow[t]{5}{*}{ Alemanha } & 1 & 0 & 2 & 0,01 & 0,01 & 0,2977 & 0,0000 & 0,0003 \\
\hline & 1 & 1 & 4 & 0,01 & 0,01 & 0,3002 & 0,0000 & 0,0001 \\
\hline & 1 & 2 & 8 & 0,01 & 0,01 & 0,2957 & 0,0000 & 0,0000 \\
\hline & 1 & 3 & 16 & 0,01 & 0,01 & 0,2992 & 0,0000 & 0,0000 \\
\hline & 1 & 4 & 32 & 0,01 & 0,01 & 0,2647 & 0,0000 & 0,0000 \\
\hline \multirow[t]{5}{*}{ Itália } & 1 & 0 & 2 & 0,75 & 0,98 & 0,1702 & 0,0000 & 0,0000 \\
\hline & 1 & 1 & 4 & 0,01 & 0,01 & 0,1717 & 0,0000 & 0,0000 \\
\hline & 1 & 2 & 8 & 0,01 & 0,01 & 0,1626 & 0,0000 & 0,0000 \\
\hline & 1 & 3 & 16 & 0,01 & 0,01 & 0,1690 & 0,0001 & 0,0002 \\
\hline & 1 & 4 & 32 & 0,01 & 0,01 & 0,2107 & 0,0002 & 0,0002 \\
\hline \multirow[t]{5}{*}{ Japão } & 1 & 0 & 2 & 0,01 & 0,01 & 0,0005 & 0,0042 & 0,0012 \\
\hline & 1 & 1 & 4 & 0,01 & 0,01 & 0,0004 & 0,0039 & 0,0001 \\
\hline & 1 & 2 & 8 & 0,01 & 0,01 & 0,0004 & 0,0042 & 0,0004 \\
\hline & 1 & 3 & 16 & 0,01 & 0,01 & 0,0004 & 0,0026 & 0,0007 \\
\hline & 1 & 4 & 32 & 0,01 & 0,01 & 0,0004 & 0,0035 & 0,0007 \\
\hline \multirow[t]{5}{*}{ Holanda } & 1 & 0 & 2 & 0,01 & 0,01 & 0,2556 & 0,0002 & 0,0003 \\
\hline & 1 & 1 & 4 & 0,36 & 0,12 & 0,2555 & 0,0008 & 0,0004 \\
\hline & 1 & 2 & 8 & 0,01 & 0,01 & 0,2474 & 0,0004 & 0,0001 \\
\hline & 1 & 3 & 16 & 0,01 & 0,01 & 0,2535 & 0,0001 & 0,0021 \\
\hline & 1 & 4 & 32 & 0,01 & 0,01 & 0,2524 & 0,0002 & 0,0001 \\
\hline Noruega & 1 & 0 & 2 & 0,01 & 0,01 & 0,7112 & 0,0000 & 0,0000 \\
\hline
\end{tabular}

(Continua na página seguinte) 
Tabela 6.2 - Continuação da página anterior

\begin{tabular}{c|c|c|c|c|c|c|c|c}
\hline País estrangeiro & $\mathrm{p}$ & $\mathrm{J}$ & N.C.O. & Teste ADF & Teste P.P. & $u_{1 t}$ & $u_{2 t}$ & $u_{3 t}$ \\
\hline & 1 & 1 & 4 & 0,01 & 0,01 & 0,7128 & 0,0000 & 0,0000 \\
& 1 & 2 & 8 & 0,01 & 0,01 & 0,7154 & 0,0000 & 0,0007 \\
& 1 & 3 & 16 & 0,01 & 0,01 & 0,7157 & 0,0000 & 0,0011 \\
& 1 & 4 & 32 & 0,01 & 0,01 & 0,7029 & 0,0000 & 0,0365 \\
\hline Espanha & 1 & 0 & 2 & 0,19 & 0,39 & 0,7776 & 0,0000 & 0,0001 \\
& 1 & 1 & 4 & 0,01 & 0,01 & 0,7711 & 0,0000 & 0,0003 \\
& 1 & 2 & 8 & 0,01 & 0,01 & 0,7730 & 0,0000 & 0,0001 \\
& 1 & 3 & 16 & 0,01 & 0,01 & 0,7713 & 0,0000 & 0,0027 \\
& 1 & 4 & 32 & 0,01 & 0,01 & 0,7995 & 0,0000 & 0,0014 \\
\hline Suécia & 1 & 0 & 2 & 0,69 & 0,85 & 0,0000 & 0,0000 & 0,0033 \\
& 1 & 1 & 4 & 0,01 & 0,01 & 0,0000 & 0,0000 & 0,0008 \\
& 1 & 2 & 8 & 0,01 & 0,01 & 0,0000 & 0,0000 & 0,0023 \\
& 1 & 3 & 16 & 0,01 & 0,01 & 0,0000 & 0,0000 & 0,0020 \\
& 1 & 4 & 32 & 0,01 & 0,01 & 0,0000 & 0,0000 & 0,5016 \\
\hline Reino Unido & 1 & 0 & 2 & 0,53 & 0,70 & 0,0065 & 0,0000 & 0,0002 \\
& 1 & 1 & 4 & 0,01 & 0,01 & 0,0063 & 0,0000 & 0,0002 \\
& 1 & 2 & 8 & 0,04 & 0,01 & 0,0065 & 0,0000 & 0,0181 \\
& 1 & 3 & 16 & 0,01 & 0,01 & 0,0058 & 0,0000 & 0,0031 \\
& 1 & 4 & 32 & 0,01 & 0,01 & 0,0069 & 0,0000 & 0,0165 \\
\hline
\end{tabular}

( ${ }^{\dagger}$ Número de coeficientes de ondaletas: N.C.O. $=2^{J+1}$.)

É possível notar pela Tabela 6.2 que a escolha de parâmetros utilizados leva a resíduos de cointegração (normalizados) que aparentemente são estacionários, conforme apontam os testes ADF e PP, tomando nível de significância de 5\%. A estacionariedade dos resíduos não é verificada apenas para Canadá, Itália, Espanha, Suécia e Reino Unido, quando $J=0$. O mesmo ocorre para a Holanda quando $J=1$. É possível notar ainda que para o Japão e Suécia, em todos os valores de $J$ considerados, não foi possível verificar a normalidade dos resíduos. Em todos os casos onde a hipótese $H 0$ de normalidade no teste de Shapiro-Wilk foi rejeitada, os resíduos pareciam apresentar caudas mais pesadas que a distribuição normal.

A seguir, são apresentados os gráficos de $\hat{\boldsymbol{\beta}}_{t}=\left[\hat{\beta}_{1 t} \hat{\beta}_{2 t} \hat{\beta}_{3 t}\right]^{\prime}$, assim como em Bierens e Martins (2010). Para apresentar os resultados, cada série foi escalonada, sendo multiplicada por $1 / 100$. Note que esta transformação não altera as relações de cointegração, que se mantém com uma multiplicação por uma constante. 
Para o modelo de PPC entre Estados Unidos e Bélgica, tomando $J=0$, apresentamos na Figura 6.2 os componentes do vetor de cointegração variando com o tempo $\hat{\boldsymbol{\beta}}_{t}=$ $\left[\hat{\beta}_{1 t} \hat{\beta}_{2 t} \hat{\beta}_{3 t}\right]^{\prime}$.

(a)

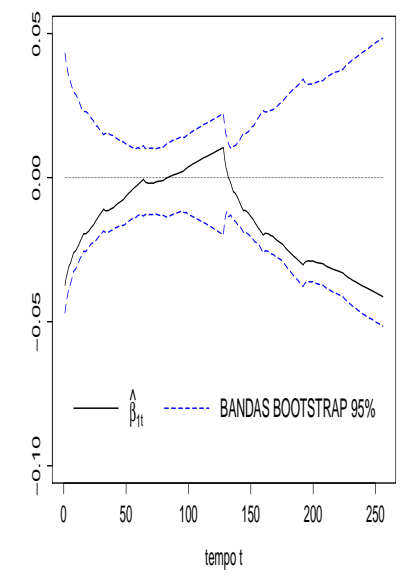

(b)

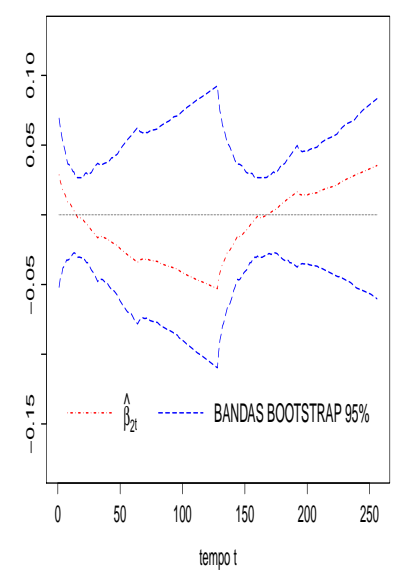

(c)

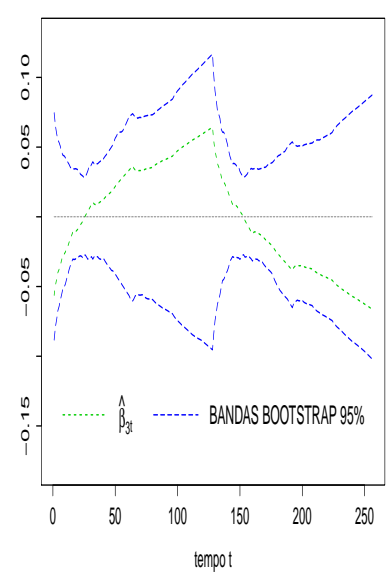

(d)

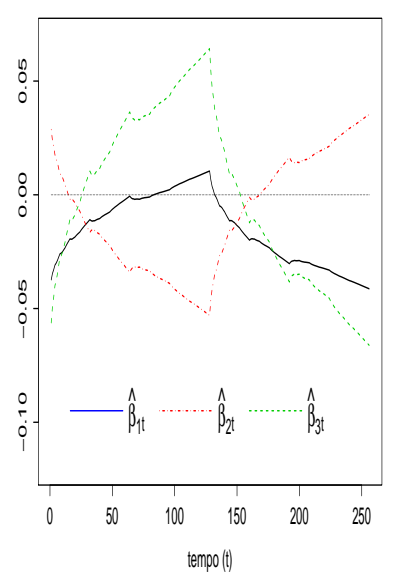

Figura 6.2: PPC entre Estados Unidos e Bélgica, tomando $J=0$ : Componentes $\hat{\beta}_{1 t}$ (a), $\hat{\beta}_{2 t}$ (b) e $\hat{\beta}_{3 t}(\mathrm{c})$ de $\hat{\boldsymbol{\beta}}_{t}=\left[\begin{array}{lll}\hat{\beta}_{1 t} & \hat{\beta}_{2 t} & \hat{\beta}_{3 t}\end{array}\right]^{\prime}$ juntamente com bandas bootstrap de 95\%. Para efeito comparativo, os três componentes são apresentadas no mesmo gráfico $(\mathrm{d})$.

Para o modelo de PPC entre Estados Unidos e Japão, tomando $J=1$, apresentamos na Figura 6.3 os componentes do vetor de cointegração variando com o tempo $\hat{\boldsymbol{\beta}}_{t}=$ $\left[\hat{\beta}_{1 t} \hat{\beta}_{2 t} \hat{\beta}_{3 t}\right]^{\prime}$.

(a)

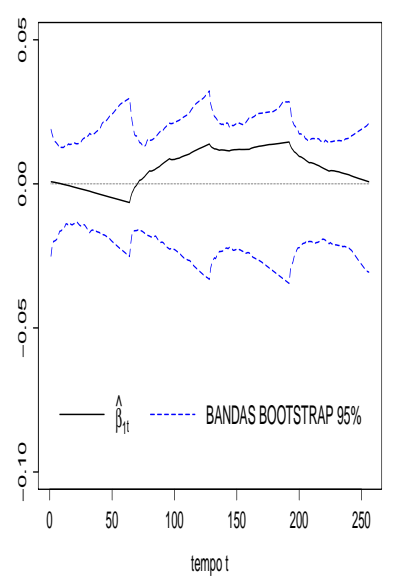

(b)

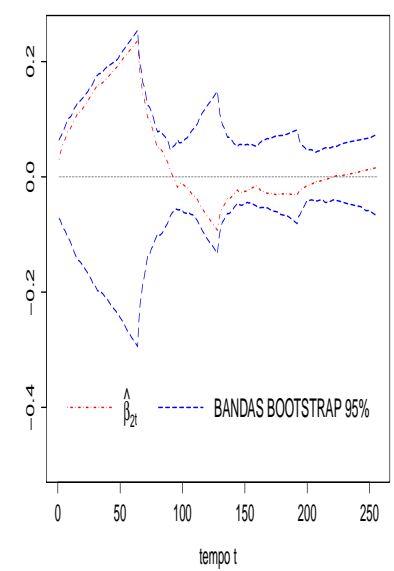

(c)

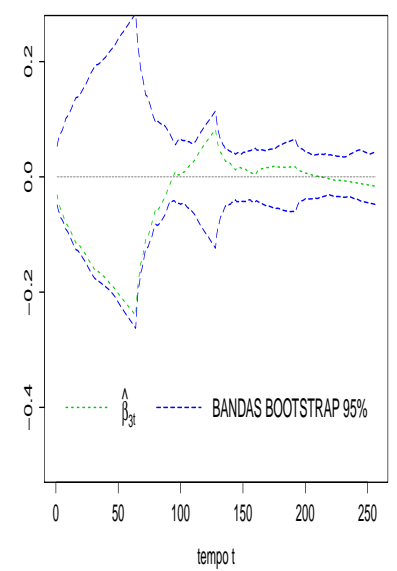

(d)

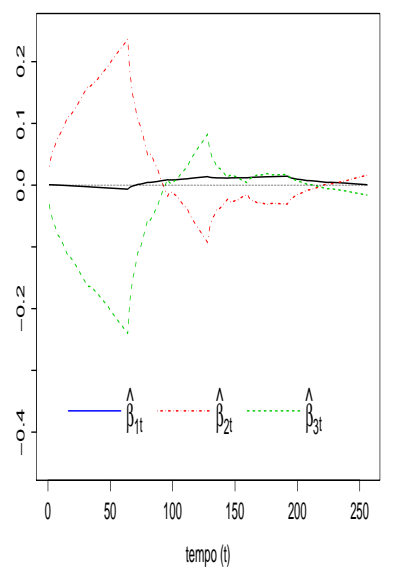

Figura 6.3: PPC entre Estados Unidos e Japão, tomando $J=1$ : Componentes $\hat{\beta}_{1 t}(\mathrm{a}), \hat{\beta}_{2 t}$ (b) e $\hat{\beta}_{3 t}(\mathrm{c})$ de $\hat{\boldsymbol{\beta}}_{t}=\left[\begin{array}{lll}\hat{\beta}_{1 t} & \hat{\beta}_{2 t} & \hat{\beta}_{3 t}\end{array}\right]^{\prime}$ juntamente com bandas bootstrap de $95 \%$. Para efeito comparativo, os três componentes são apresentadas no mesmo gráfico $(\mathrm{d})$. 
Para o modelo de PPC entre Estados Unidos e Noruega, tomando $J=2$, apresentamos na Figura 6.4 os componentes do vetor de cointegração variando com o tempo $\hat{\boldsymbol{\beta}}_{t}=$ $\left[\hat{\beta}_{1 t} \hat{\beta}_{2 t} \hat{\beta}_{3 t}\right]^{\prime}$.

(a)

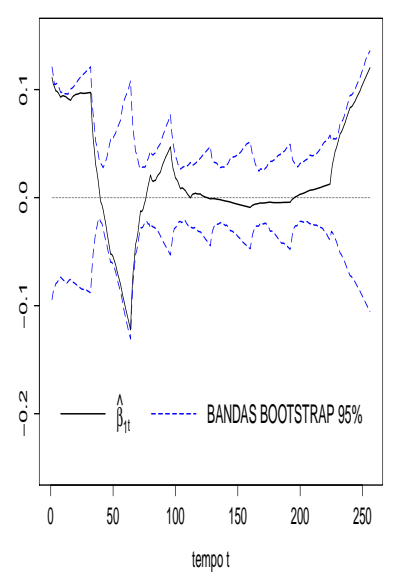

(b)

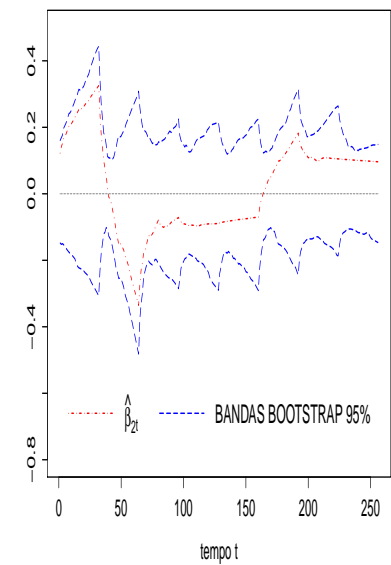

(c)

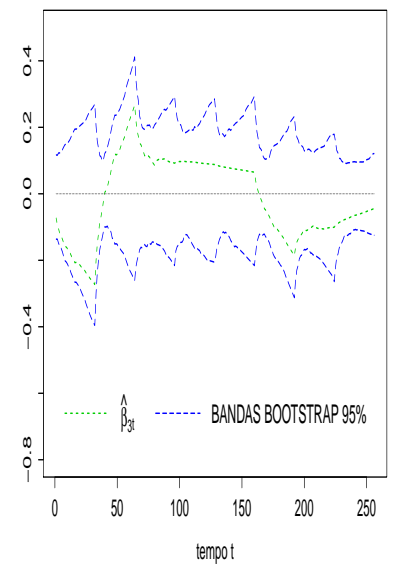

(d)

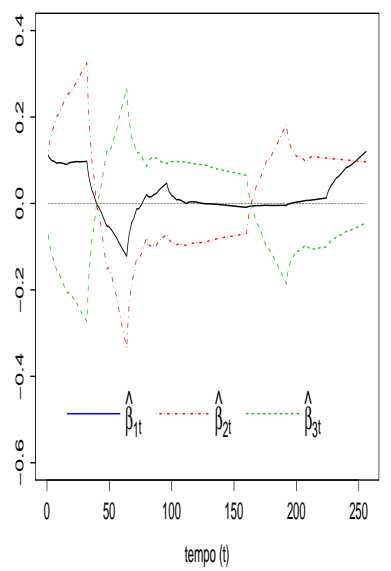

Figura 6.4: PPC entre Estados Unidos e Noruega, tomando $J=2$ : Componentes $\hat{\beta}_{1 t}$ (a), $\hat{\beta}_{2 t}$ (b) e $\hat{\beta}_{3 t}$ (c) de $\hat{\boldsymbol{\beta}}_{t}=\left[\hat{\beta}_{1 t} \hat{\beta}_{2 t} \hat{\beta}_{3 t}\right]^{\prime}$ juntamente com bandas bootstrap de $95 \%$. Para efeito comparativo, os três componentes são apresentadas no mesmo gráfico (d).

Para o modelo de PPC entre Estados Unidos e Alemanha, tomando $J=3$, apresentamos na Figura 6.5 os componentes do vetor de cointegração variando com o tempo $\hat{\boldsymbol{\beta}}_{t}=\left[\begin{array}{lll}\hat{\beta}_{1 t} & \hat{\beta}_{2 t} & \hat{\beta}_{3 t}\end{array}\right]^{\prime}$.

(a)

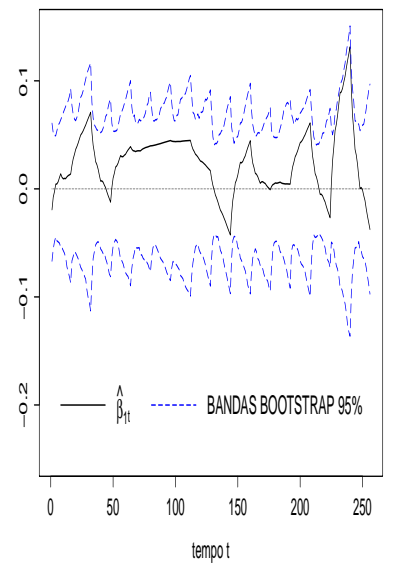

(b)

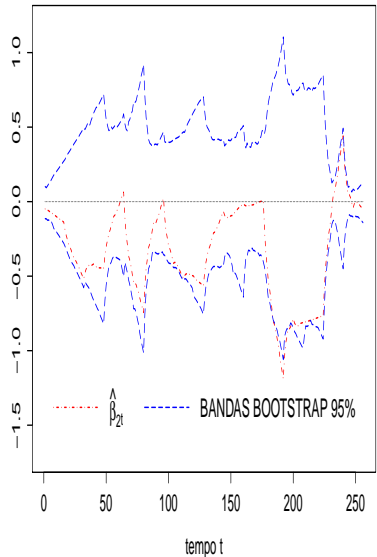

(c)

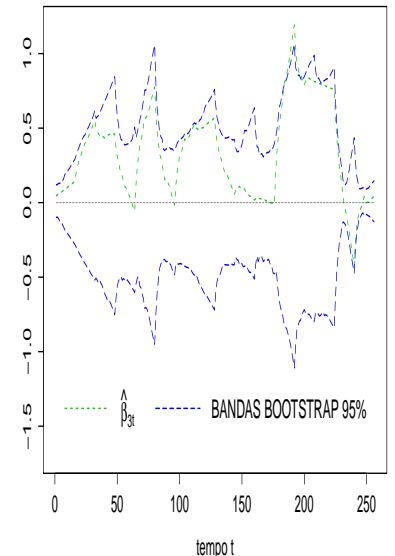

(d)

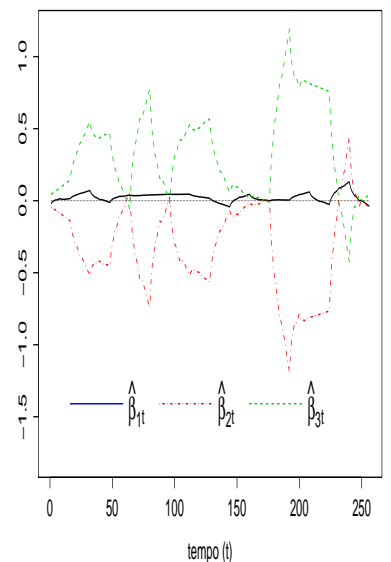

Figura 6.5: PPC entre Estados Unidos e Alemanha, tomando $J=3$ : Componentes $\hat{\beta}_{1 t}$ (a), $\hat{\beta}_{2 t}$ (b) e $\hat{\beta}_{3 t}$ (c) de $\hat{\boldsymbol{\beta}}_{t}=\left[\hat{\beta}_{1 t} \hat{\beta}_{2 t} \hat{\beta}_{3 t}\right]^{\prime}$ juntamente com bandas bootstrap de $95 \%$. Para efeito comparativo, os três componentes são apresentadas no mesmo gráfico (d). 
A Tabela 6.3 apresenta as estimativas da matriz de cargas $\boldsymbol{\alpha}^{*}=\left[\begin{array}{lll}\alpha_{11}^{*} & \alpha_{21}^{*} & \alpha_{31}^{*}\end{array}\right]$ para os modelos ajustados na Tabela 6.1 , com $J=4$ (N.C.O.=32). Pela tabela é possível verificar que em todos os casos, as componentes de $\boldsymbol{\alpha}^{*}$ são significativamente iguais a zero. É possível ainda concluir que a escolha da defasagem $p=1$, como em Martins (2016), além de não eliminar a autocorrelação presente na série de resíduos, não parece trazer um bom ajuste para os modelos.

Tabela 6.3: Estimativa e medidas bootstrap $\left(^{\star}\right)$ para a matriz de cargas $\boldsymbol{\alpha}$.

\begin{tabular}{|c|c|c|c|c|c|c|}
\hline País estrangeiro & $\mathrm{p}$ & $\mathrm{J}$ & $\hat{\boldsymbol{\alpha}}^{*}$ & I.C. ${ }^{\star}$ & Média* & Erro Padrão \\
\hline \multirow[t]{3}{*}{ Bélgica } & 1 & 4 & 0,0006 & {$[-0,0047 ; 0,0000]$} & 0,0002 & 0,0015 \\
\hline & 1 & 4 & $-0,0001$ & {$[-0,0034 ; 0,0066]$} & 0,0005 & 0,0023 \\
\hline & 1 & 4 & $-0,0001$ & {$[-0,0053 ; 0,0101]$} & 0,0008 & 0,0036 \\
\hline \multirow[t]{3}{*}{ Canadá } & 1 & 4 & 0,0002 & {$[-0,0059 ; 0,0000]$} & $-0,0005$ & 0,0017 \\
\hline & 1 & 4 & $-0,0002$ & {$[-0,0237 ; 0,0102]$} & $-0,0027$ & 0,0075 \\
\hline & 1 & 4 & $-0,0003$ & {$[-0,0247 ; 0,0107]$} & $-0,0029$ & 0,0078 \\
\hline \multirow[t]{3}{*}{ Dinamarca } & 1 & 4 & $-0,00004$ & {$[-0,0045 ; 0,0000]$} & 0,00000 & 0,0016 \\
\hline & 1 & 4 & $-0,00001$ & {$[-0,0058 ; 0,0053]$} & 0,00004 & 0,0026 \\
\hline & 1 & 4 & $-0,00001$ & {$[-0,0057 ; 0,0053]$} & 0,00004 & 0,0025 \\
\hline \multirow[t]{3}{*}{ França } & 1 & 4 & 0,0002 & {$[-0,0073 ; 0,0000]$} & 0,0007 & 0,0026 \\
\hline & 1 & 4 & $-0,0001$ & {$[-0,0078 ; 0,0133]$} & 0,0015 & 0,0049 \\
\hline & 1 & 4 & 0,0000 & {$[-0,0069 ; 0,0117]$} & 0,0013 & 0,0043 \\
\hline \multirow[t]{3}{*}{ Alemanha } & 1 & 4 & 0,0003 & {$[-0,0097 ; 0,0000]$} & $-0,0001$ & 0,0032 \\
\hline & 1 & 4 & 0,0000 & {$[-0,0107 ; 0,0064]$} & $-0,0001$ & 0,0037 \\
\hline & 1 & 4 & 0,0000 & {$[-0,0206 ; 0,0126]$} & $-0,0002$ & 0,0072 \\
\hline \multirow[t]{3}{*}{ Itália } & 1 & 4 & 0,0017 & {$[-0,0044 ; 0,0000]$} & 0,0000 & 0,0016 \\
\hline & 1 & 4 & 0,0000 & {$[-0,0045 ; 0,0070]$} & 0,0002 & 0,0027 \\
\hline & 1 & 4 & 0,0000 & {$[-0,0026 ; 0,0038]$} & 0,0001 & 0,0015 \\
\hline \multirow[t]{3}{*}{ Japão } & 1 & 4 & $-0,0011$ & {$[-0,0043 ; 0,0000]$} & $-0,0004$ & 0,0014 \\
\hline & 1 & 4 & 0,0009 & {$[-0,0018 ; 0,0079]$} & 0,0012 & 0,0024 \\
\hline & 1 & 4 & 0,0021 & {$[-0,0040 ; 0,0177]$} & 0,0027 & 0,0053 \\
\hline \multirow[t]{3}{*}{ Holanda } & 1 & 4 & $-0,0002$ & {$[-0,0031 ; 0,0000]$} & 0,0006 & 0,0009 \\
\hline & 1 & 4 & $-0,0001$ & {$[-0,0035 ; 0,0194]$} & 0,0039 & 0,0058 \\
\hline & 1 & 4 & $-0,0003$ & {$[-0,0057 ; 0,0319]$} & 0,0065 & 0,0096 \\
\hline \multirow[t]{3}{*}{ Noruega } & 1 & 4 & 0,0000 & {$[-0,0050 ; 0,0000]$} & $-0,0001$ & 0,0015 \\
\hline & 1 & 4 & 0,0000 & {$[-0,0110 ; 0,0110]$} & 0,0002 & 0,0053 \\
\hline & 1 & 4 & 0,0000 & {$[-0,0100 ; 0,0101]$} & 0,0002 & 0,0047 \\
\hline \multirow[t]{3}{*}{ Espanha } & 1 & 4 & 0,0000 & {$[-0,0050 ; 0,0000]$} & $-0,0001$ & 0,0015 \\
\hline & 1 & 4 & 0,0000 & {$[-0,0110 ; 0,0110]$} & 0,0002 & 0,0053 \\
\hline & 1 & 4 & 0,0000 & {$[-0,0100 ; 0,0101]$} & 0,0002 & 0,0047 \\
\hline \multirow[t]{3}{*}{ Suécia } & 1 & 4 & $-0,0005$ & {$[-0,0058 ; 0,0000]$} & $-0,0005$ & 0,0020 \\
\hline & 1 & 4 & $-0,0011$ & {$[-0,0104 ; 0,0063]$} & $-0,0009$ & 0,0038 \\
\hline & 1 & 4 & $-0,0007$ & {$[-0,0105 ; 0,0061]$} & $-0,0009$ & 0,0036 \\
\hline \multirow[t]{3}{*}{ Reino Unido } & 1 & 4 & $-0,0005$ & {$[-0,0038 ; 0,0000]$} & $-0,0001$ & 0,0013 \\
\hline & 1 & 4 & $-0,0010$ & {$[-0,0100 ; 0,0112]$} & $-0,0002$ & 0,0044 \\
\hline & 1 & 4 & $-0,0012$ & {$[-0,0073 ; 0,0080]$} & $-0,0002$ & 0,0032 \\
\hline
\end{tabular}




\subsubsection{Ajuste com defasagem $p=13$ e ondaletas Daubechies D10}

Conforme comentado anteriormente, Bierens e Martins (2010) e Martins (2016) utilizam apenas a defasagem $p=1$ em suas análises. No entanto, a escolha de tal defasagem deixa considerável correlação nos resíduos dos modelos ajustados. Tal fato pode ser verificado na Figura 6.6, para o modelo de cointegração entre Estados Unidos e Canadá, tomando $J=2, p=1$ e base de ondaletas Daubechies D10.
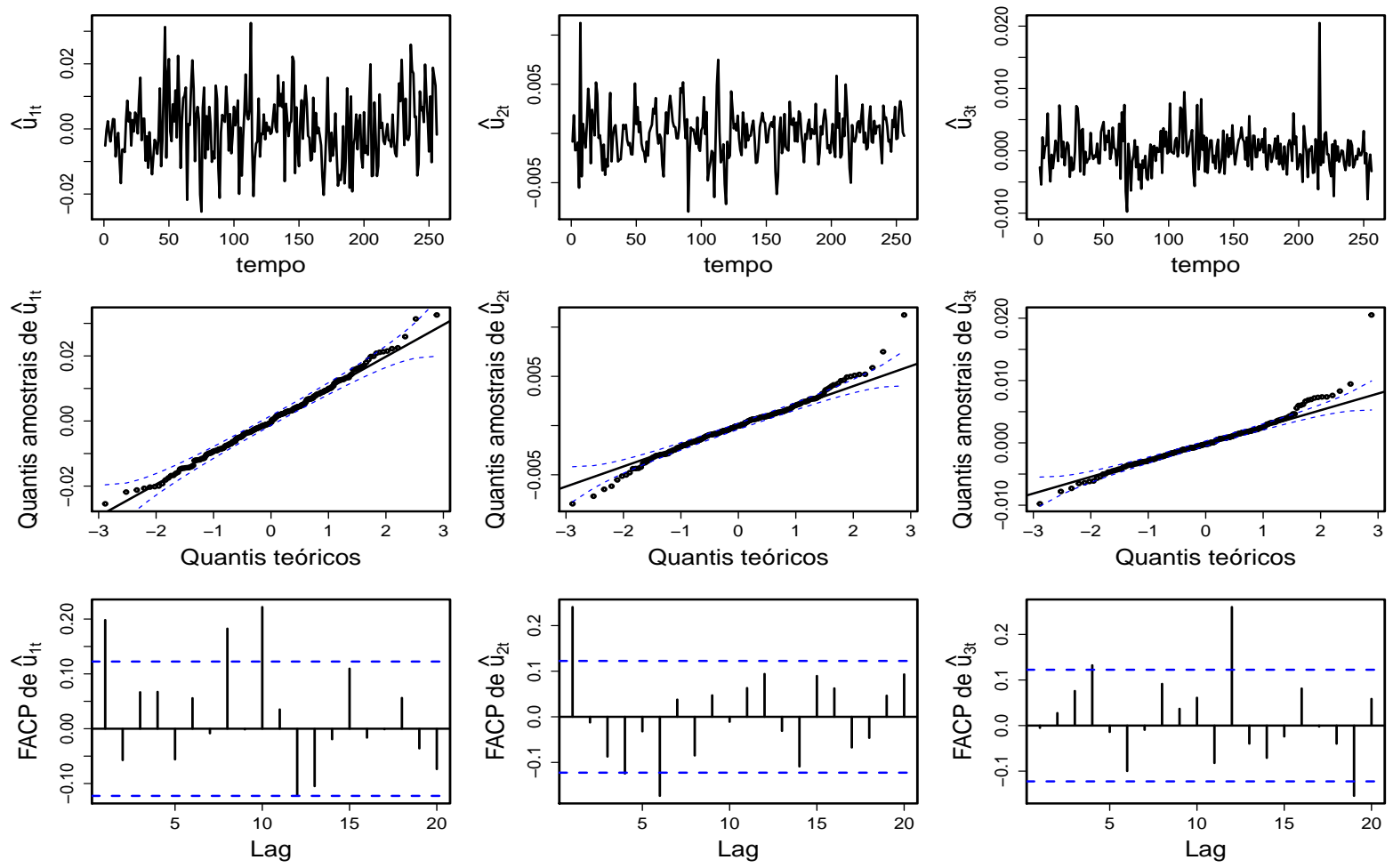

Figura 6.6: PPC entre Estados Unidos e Canadá: Resíduos estimados pelo modelo, quantis amostrais e função de autocorrelação parcial (FACP) de $\hat{u}_{t}=\left[\begin{array}{lll}\hat{u}_{1 t} & \hat{u}_{2 t} & \hat{u}_{3 t}\end{array}\right]^{\prime}$.

Em todos os modelos considerados na Tabela 6.1, a escolha do parâmetro $J$ não influenciou significativamente a correlação presente na série de resíduos. Além disso, Bierens e Martins (2010) e Martins (2016) não deixam claro se modelar o vetor de cointegração utilizando polinômios de Chebyschev é suficiente para eliminar a correlação presente nos resíduos dos modelos ajustados.

Para o modelo de PPC entre Estados Unidos e Canadá, são apresentados na Figura 6.7 os resíduos $\hat{u}_{1 t}, \hat{u}_{2 t}$ e $\hat{u}_{3 t}$ de um novo modelo ajustado, considerando $p=13$ e $J=2$. O teste de Shapiro-Wilk foi aplicado individualmente a $\hat{u}_{1 t}, \hat{u}_{2 t}$ e $\hat{u}_{3 t}$, fornecendo os respectivos valores- $p$ : 0,9910, 0,0017 e 0,0000. Desta forma, é possível validar a suposição de normalidade apenas para a componente $\hat{u}_{1 t}$. Tal fato é confirmado pelos QQ-plots apresentados em 6.7. Note também que o desvio da normalidade é bastante perceptível nas caudas de $\hat{u}_{2 t}$ e $\hat{u}_{3 t}$. Os desvios de normalidade dos resíduos apresentados na Tabela 6.1 são similares aos apresentados por $\hat{u}_{2 t}$ e $\hat{u}_{3 t}$ em 6.7 . 

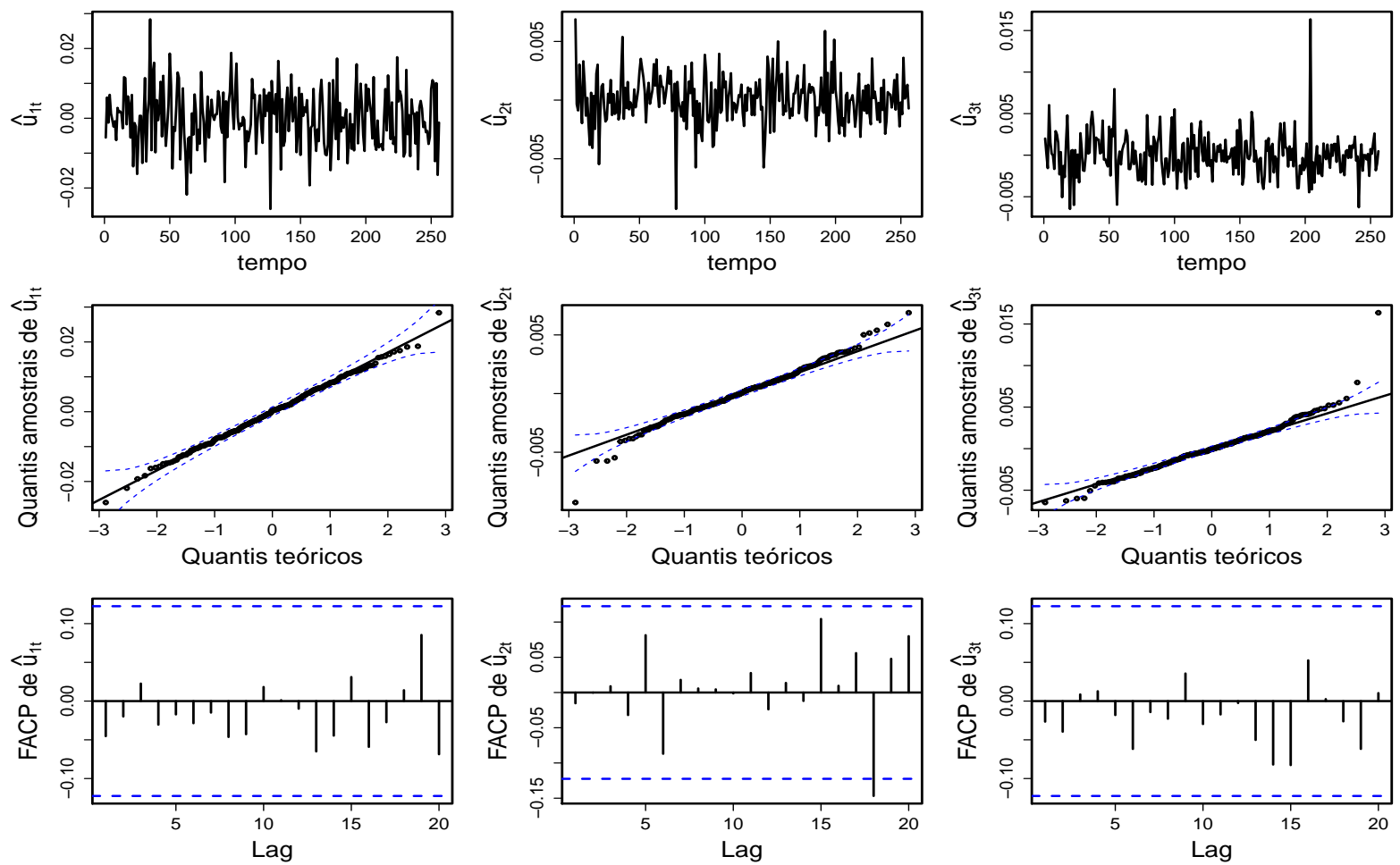

Figura 6.7: PPC entre Estados Unidos e Canadá: Resíduos estimados pelo modelo, quantis amostrais e função de autocorrelação parcial (FACP) de $\hat{u}_{t}=\left[\begin{array}{lll}\hat{u}_{1 t} & \hat{u}_{2 t} & \hat{u}_{3 t}\end{array}\right]^{\prime}$.

Na Figura 6.8 (a), são apresentados os resíduos de cointegração estimados $\hat{e}_{t}=\hat{\boldsymbol{\beta}}_{t}^{\prime} Y_{t}$, $\operatorname{com} \hat{\boldsymbol{\beta}}_{t}=\left[\begin{array}{lll}\hat{\beta}_{1 t} & \hat{\beta}_{2 t} & \hat{\beta}_{3 t}\end{array}\right]^{\prime}$. Para esta série foram obtidos os respectivos valores- $p$ dos testes ADF e PP: 0.2766 e 0.5568 . Na Figura 6.8 (b) são apresentados os resíduos normalizados $\hat{e}_{t}^{*}=\hat{\boldsymbol{\beta}}_{t}^{*^{\prime}} Y_{t}$, com $\boldsymbol{\beta}_{t}=\left[\begin{array}{ccc}\hat{\beta}_{1 t}^{*} & \hat{\beta}_{2 t}^{*} & \hat{\beta}_{3 t}^{*}\end{array}\right]^{\prime}$, com $\hat{\beta}_{1 t}^{*}=1, \forall t$. Para esta série foram obtidos os respectivos valores- $p$ dos testes ADF e PP: 0,01 e 0.01. Tomando um nível de significância de $5 \%$, foi possível então concluir que $\hat{e}_{t}^{*}$ é estacionário.

(a)

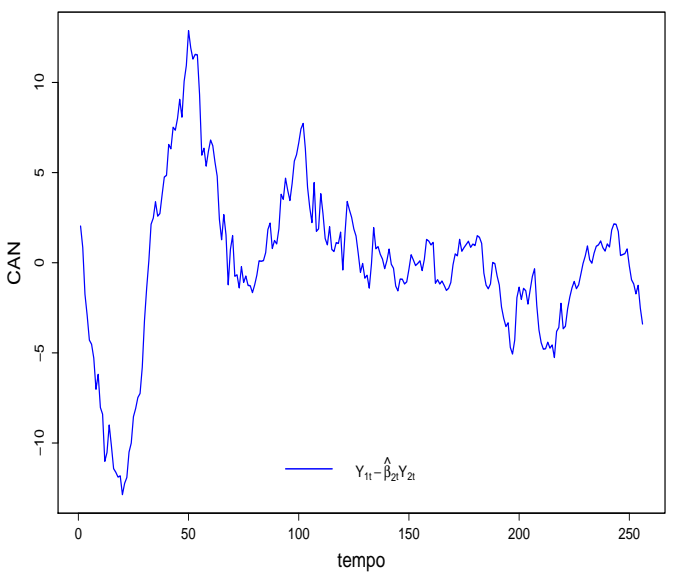

(b)

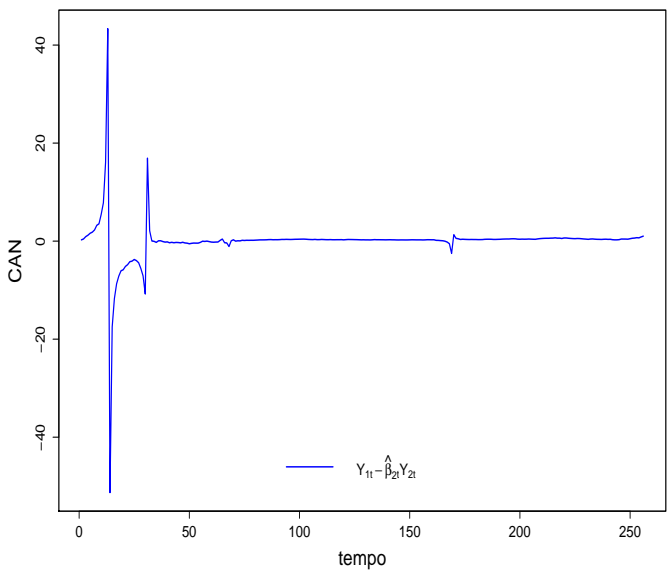

Figura 6.8: PPC entre Estados Unidos e Canadá: Série de resíduos de cointegração não normalizados $\hat{e}_{t}(\mathbf{a})$ e normalizados $\hat{e}_{t}^{*}(\mathbf{b})$. 
Na Figura 6.9 são apresentadas as estimativas para os componentes $\hat{\beta}_{1 t}$ (a), $\hat{\beta}_{2 t}$ (b) e $\hat{\beta}_{2 t}$ (c) do vetor de cointegração variando com o tempo $\hat{\boldsymbol{\beta}}_{t}=\left[\hat{\beta}_{1 t} \hat{\beta}_{2 t} \hat{\beta}_{3 t}\right]^{\prime}$, juntamente com as bandas de confiança geradas a partir de simulações bootstrap. Cada gráfico contém as bandas de confiança bootstrap de $95 \%$. Além disso, em 6.9 (d) apresentamos $\hat{\beta}_{1 t}, \hat{\beta}_{2 t}$ e $\hat{\beta}_{3 t}$ conjuntamente, com o objetivo de comparar os componentes ao longo do tempo.

(a) $\hat{\beta}_{1 t}$

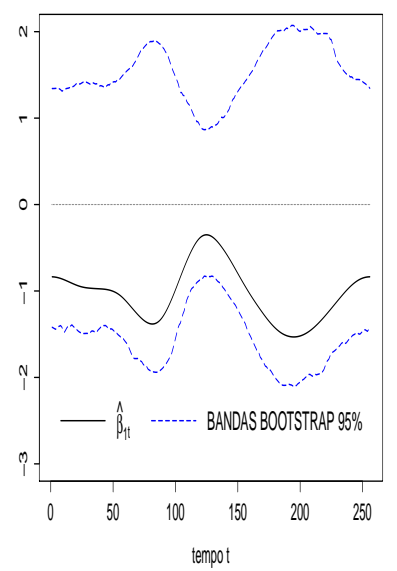

(b) $\hat{\beta}_{2 t}$

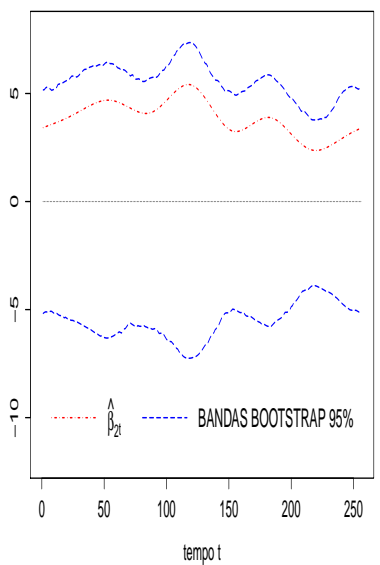

(c) $\hat{\beta}_{3 t}$

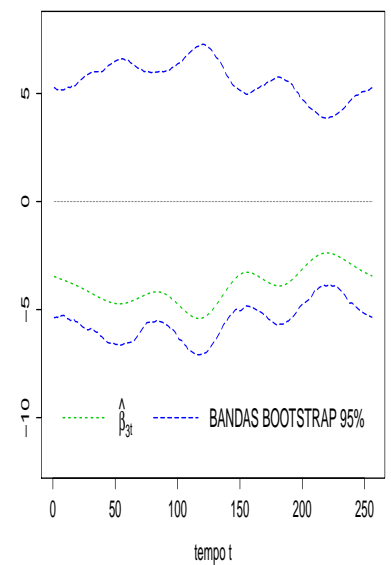

(d) $\hat{\beta}_{1 t}, \hat{\beta}_{2 t}$ e $\hat{\beta}_{3 t}$

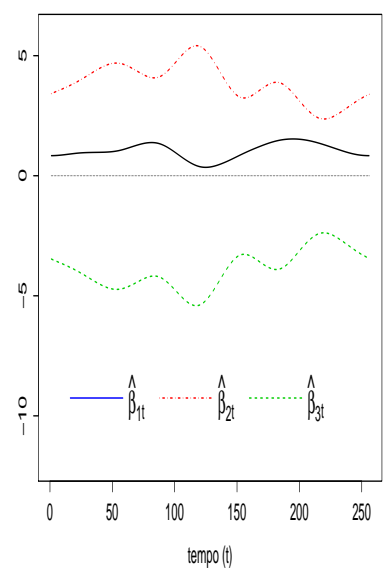

Figura 6.9: PPC entre Estados Unidos e Canadá: Componentes $\hat{\beta}_{1 t}$ (a), $\hat{\beta}_{2 t}$ (b) e $\hat{\beta}_{3 t}$ (c) de $\hat{\boldsymbol{\beta}}_{t}=\left[\begin{array}{lll}\hat{\beta}_{1 t} & \hat{\beta}_{2 t} & \hat{\beta}_{3 t}\end{array}\right]^{\prime}$ juntamente com bandas bootstrap de $95 \%$. Para efeito comparativo, os três componentes são apresentadas no mesmo gráfico $(\mathrm{d})$.

Na Figura 6.10 são apresentadas as estimativas para os componentes $\hat{\beta}_{1 t}^{*}(\mathrm{a}), \hat{\beta}_{2 t}^{*}$ (b) e $\hat{\beta}_{3 t}^{*}$ (c) do vetor de cointegração normalizado $\hat{\boldsymbol{\beta}}_{t}^{*}=\left[\hat{\beta}_{1 t}^{*} \hat{\beta}_{2 t}^{*} \hat{\beta}_{3 t}^{*}\right]^{\prime}$, juntamente com as bandas de confiança geradas a partir de simulações bootstrap.

(a) $\hat{\beta}_{1 t}$

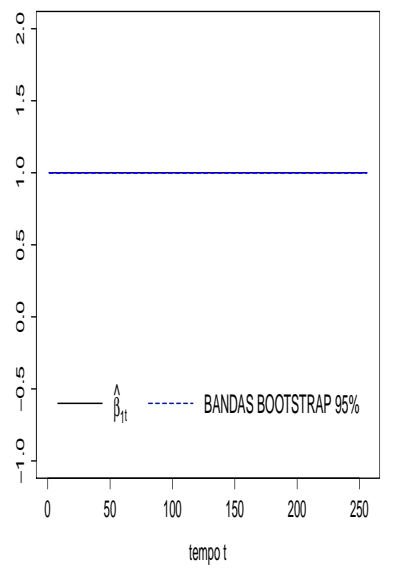

(b) $\hat{\beta}_{2 t}$

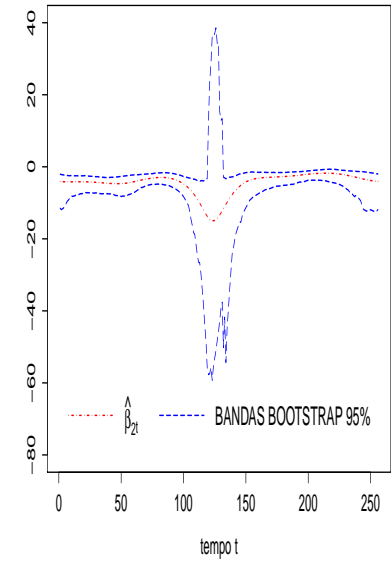

(c) $\hat{\beta}_{3 t}$

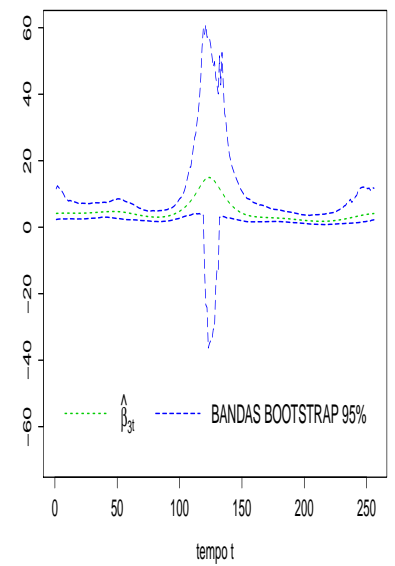

(d) $\hat{\beta}_{1 t}, \hat{\beta}_{2 t}$ e $\hat{\beta}_{3 t}$

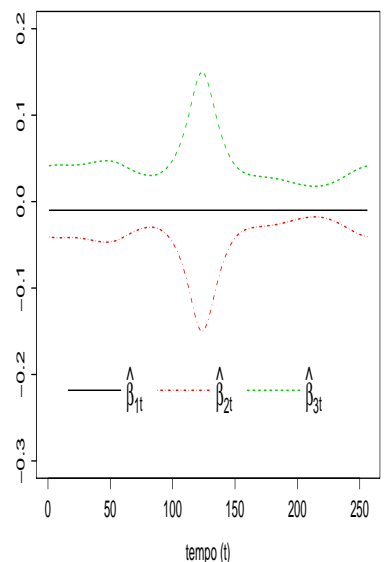

Figura 6.10: PPC entre Estados Unidos e Canadá: Componentes $\hat{\beta}_{1 t}$ (a), $\hat{\beta}_{2 t}$ (b) e $\hat{\beta}_{3 t}$ (c) de $\hat{\boldsymbol{\beta}}_{t}=\left[\begin{array}{lll}\hat{\beta}_{1 t} & \hat{\beta}_{2 t} & \hat{\beta}_{3 t}\end{array}\right]^{\prime}$ juntamente com bandas bootstrap de 95\%. Para efeito comparativo, os três componentes são apresentadas no mesmo gráfico (d). 
Na Figura 6.11, apresentamos a estatística $R V_{C V T}^{(J)}$ calculada sob $H 0$ (a) e sob $H A$ (b). Rejeitamos a hipótese de coitegração constante ao longo do tempo, com valor- $p^{\star}=0,0050$ (obtido via bootstrap).

(a)

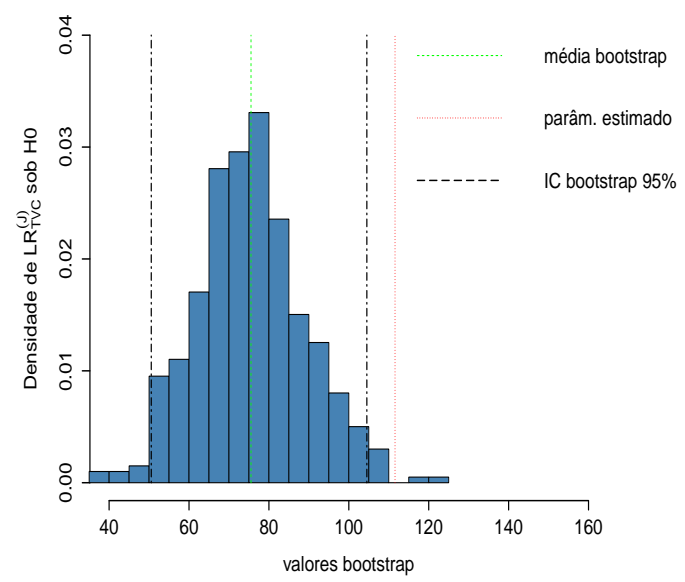

(b)

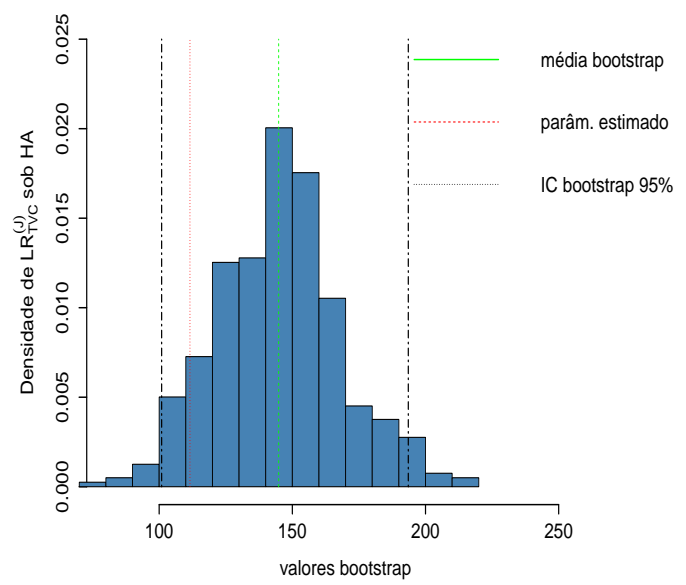

Figura 6.11: PPC entre Estados Unidos e Canadá: Histograma, densidade e quantis bootstrap $2,5 \%$ e $97,5 \%$ para $R V_{C V T}^{(J)}$ sob H0 (vetor de cointegração constante) (a) e sob HA (b).

A Figura 6.12 contém os histogramas bootstrap dos componentes do parâmetro (matricial) $\hat{\boldsymbol{\alpha}}^{*}=\left[\begin{array}{lll}\hat{\alpha}_{11}^{*} & \hat{\alpha}_{21}^{*} & \hat{\alpha}_{31}^{*}\end{array}\right]^{\prime}$ do modelo. Podemos notar em todas as figuras que os valores teóricos dos parâmetros estão dentro dos intervalos de confiança bootstrap de $95 \%$. Pelas figuras ainda é possível comparar a estimativa de cada parâmetro do modelo com sua respectiva média boostrap.

(a)

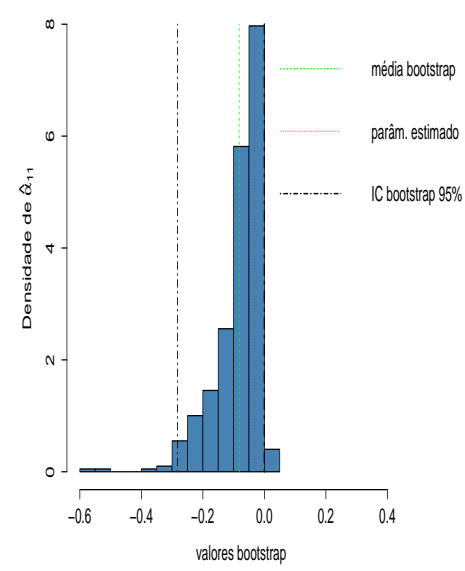

(b)

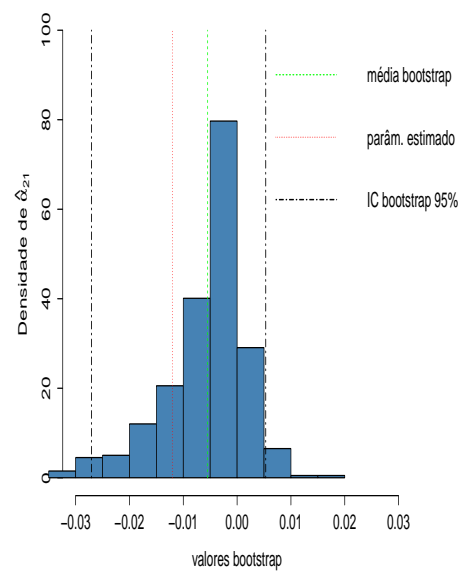

(c)

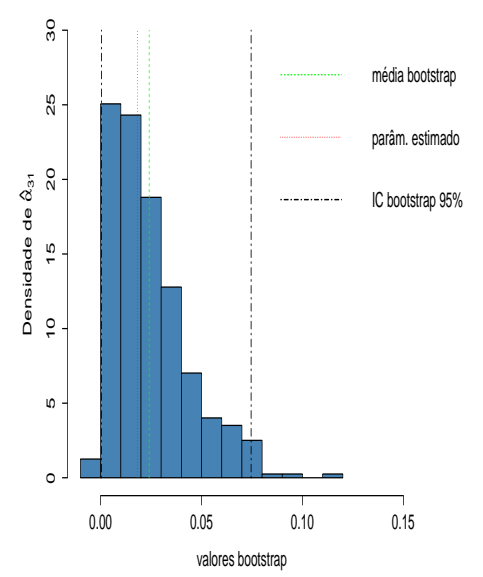

Figura 6.12: PPC entre Estados Unidos e Canadá: Histograma, densidade e quantis bootstrap $2,5 \%$ e $97,5 \%$ para as componentes $\hat{\alpha}_{11}^{*}(\mathbf{a}), \hat{\alpha}_{21}^{*}(\mathbf{b})$ e $\hat{\alpha}_{31}^{*}(\mathbf{c}) \operatorname{de} \hat{\boldsymbol{\alpha}}^{*}=\left[\begin{array}{lll}\hat{\alpha}_{11}^{*} & \hat{\alpha}_{21}^{*} & \hat{\alpha}_{31}^{*}\end{array}\right]^{\prime}$. 
A Tabela 6.4 apresenta as estimativas da estatística $R V_{C V T}^{(J)}$, os intervalos de confiança e o valor-p bootstrap. Note que a tabela complementa as informações da Figura 6.11.

Tabela 6.4: Estimativa e medidas bootstrap ${ }^{\star}$ sob H0 (vetor de cointegração constante).

\begin{tabular}{c|ccccc}
\hline Parâmetro & Estimativa & Intervalo de confiança & Média $^{\star}$ & Erro Padrão $^{\star}$ & valor- $p^{\star}$ \\
\hline$R V_{C V T}^{(J)}(\mathrm{H} 0)$ & 111,5649 & {$[50,55002 ; 104,5119]$} & 75,51765 & 13,4707 & 0,0050 \\
\hline
\end{tabular}

A Tabela 6.5 apresenta as estimativas dos parâmetros, bem como medidas bootstrap de interesse. Note que a tabela complementa as informações das Figuras 6.11 e 6.12.

Tabela 6.5: Estimativas e medidas bootstrap ${ }^{\star}$ sob HA (três escalas ao longo do tempo).

\begin{tabular}{c|cccc}
\hline Parâmetro & Estimativa & Intervalo de confiança & Média $^{\star}$ & Erro Padrão $^{\star}$ \\
\hline$R V_{C V T}^{(J)}(\mathrm{HA})$ & 111,5649 & {$[100,9266 ; 193,5375]$} & 144,8722 & 23,35871 \\
\hline$\alpha_{11}^{*}$ & $-0,0309$ & {$[-0,2825 ;-0,0003]$} & $-0,0818$ & 0,0777 \\
$\alpha_{21}^{*}$ & $-0,0120$ & {$[-0,0270 ; 0,0053]$} & $-0,0055$ & 0,0076 \\
$\alpha_{31}^{*}$ & 0,0182 & {$[0,0004 ; 0,0746]$} & 0,0240 & 0,0194 \\
\hline$\sigma_{11}^{*}=\left(\times 10^{4}\right)$ & 0,8039 & {$[0,4814 ; 0,9576]$} & 0,6939 & 0,1213 \\
$\sigma_{12}^{*}=\sigma_{21}^{*}\left(\times 10^{4}\right)$ & $-0,0052$ & {$[-0,0129 ; 0,0334]$} & 0,0093 & 0,0119 \\
$\sigma_{13}^{*}=\sigma_{31}^{*}\left(\times 10^{4}\right)$ & $-0,0150$ & {$[-0,0787 ;-0,0044]$} & $-0,0394$ & 0,0192 \\
$\sigma_{22}^{*}=\left(\times 10^{4}\right)$ & 0,0416 & {$[0,0232 ; 0,0547]$} & 0,0369 & 0,0085 \\
$\sigma_{23}^{*}=\sigma_{32}^{*}\left(\times 10^{4}\right)$ & 0,0114 & {$[-0,0102 ; 0,0030]$} & $-0,0032$ & 0,0033 \\
$\sigma_{33}^{*}=\left(\times 10^{4}\right)$ & 0,0757 & {$[0,0422 ; 0,1110]$} & 0,0677 & 0,0184 \\
\hline
\end{tabular}

É possível notar pela Tabela 6.5 que, diferente de $\hat{\alpha}_{21}^{*}$, os componentes $\hat{\alpha}_{11}^{*}$ e $\hat{\alpha}_{31}^{*}$ são significativamente diferentes de zero, considerando um nível de confiança de $5 \%$ para a construção do intervalo de confiança bootstrap.

No entanto, vale ressaltar que em diversos modelos ajustados e apresentados na Tabela 6.1 , foram encontrados $\hat{\alpha}_{11}^{*}, \hat{\alpha}_{21}^{*}$ e $\hat{\alpha}_{31}^{*}$ significativamente iguais a zero, ou seja, dentro dos intervalos de confiança obtidos nas simulações bootstrap. Bierens e Martins (2010) e Martins (2016) sequer apresentam seus resultados para as estimativas destes parâmetros. Logo, não é possível concluir se resultados similares foram obtidos ou se os autores realmente não se preocuparam com as estimativas deste parâmetro.

É possível ainda notar pela Figura 6.10 que as condições empregadas em Martins (2016) para que haja cointegração foram satisfeitas, ou seja: pudemos concluir que $\hat{e}_{t}^{*}$ é estacionária e $\beta_{2 t}<0$ e $\beta_{3 t}>0$ para os valores de $t$ considerados. 


\subsection{Paridade de Poder de Compra com coeficientes variando com o tempo - caso com intercepto}

São apresentados a seguir os ajustes de modelos considerando o intercepto para as séries de Canadá e França do conjunto de dados. Em cada caso, foram escolhidos valores de $p$, parâmetro de ondaleta $J$ e base de ondaletas convenientes, de forma a encontrar séries de resíduos sem correlação serial. A ausência de correlação serial visa a obtenção de melhores resultados para as bandas bootstrap. Em cada caso, desejamos verificar se o intercepto varia com o tempo, de acordo com as interpretações para a estimativa de $e^{\eta_{t}}$, apresentadas na Seção 4.7.

Apresentamos nas Figuras 6.13 e 6.14 as estimativas para o termo $e^{\eta_{t}}$ do modelo de PPC cointegrado para os interceptos dos modelos aplicados às séries de Canadá e França. Na Tabela 6.6 por sua vez, verificamos a estacionariedade dos resíduos de cointegração e a suposição de normalidade do modelo. A escolha das defasagens $p$ em cada modelo refere-se a uma das defasagens utilizada em Martins (2016) em seus ajustes.

Intercepto para modelo de contegração: CAN vs. USA

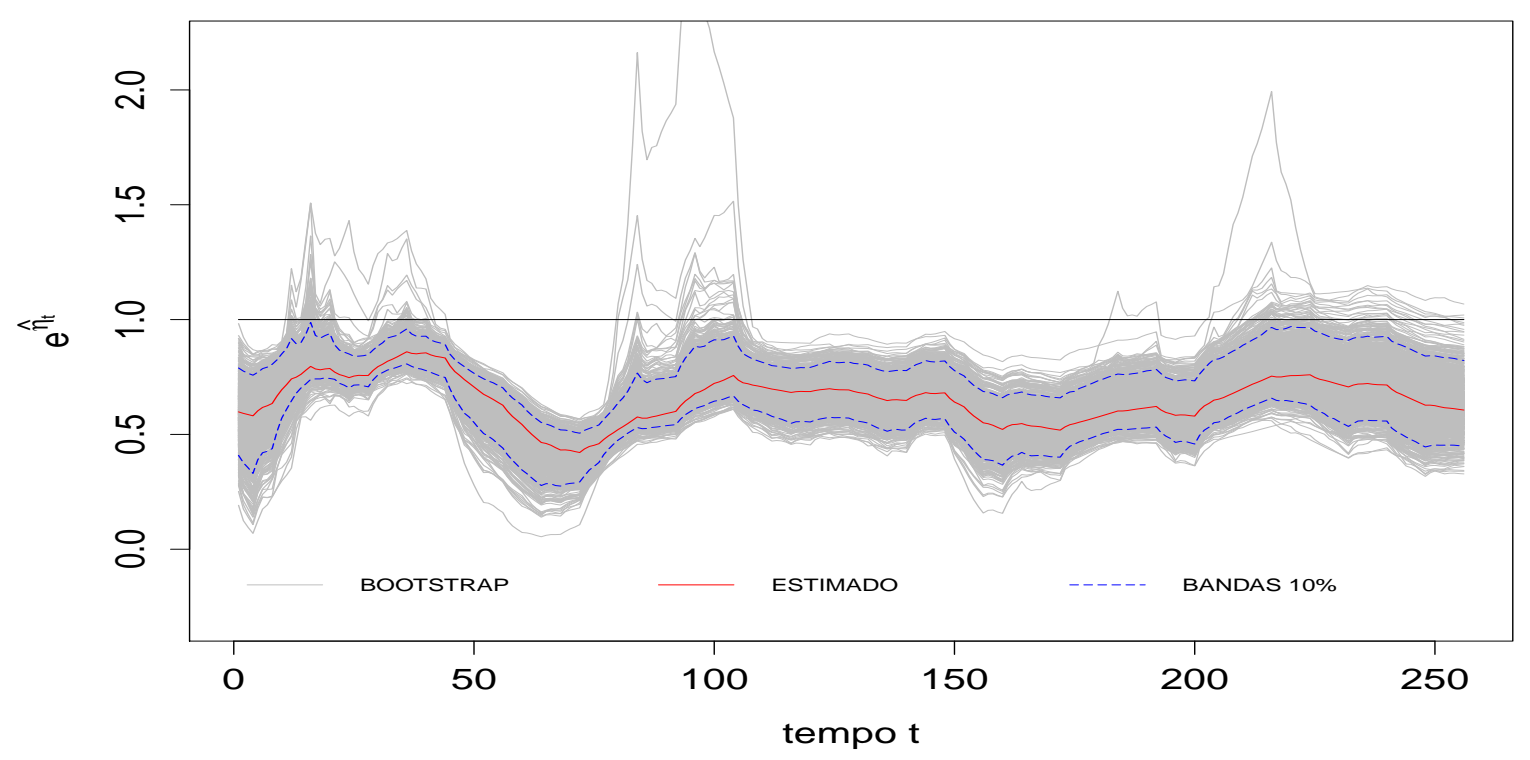

Figura 6.13: Gráficos com quantidade $e^{\eta_{t}}$ estimada, juntamente com bandas de confiança, para os países estrangeiros Bélgica, Canadá, Dinamarca, França. 
Intercepto para modelo de contegração: FRA vs. USA

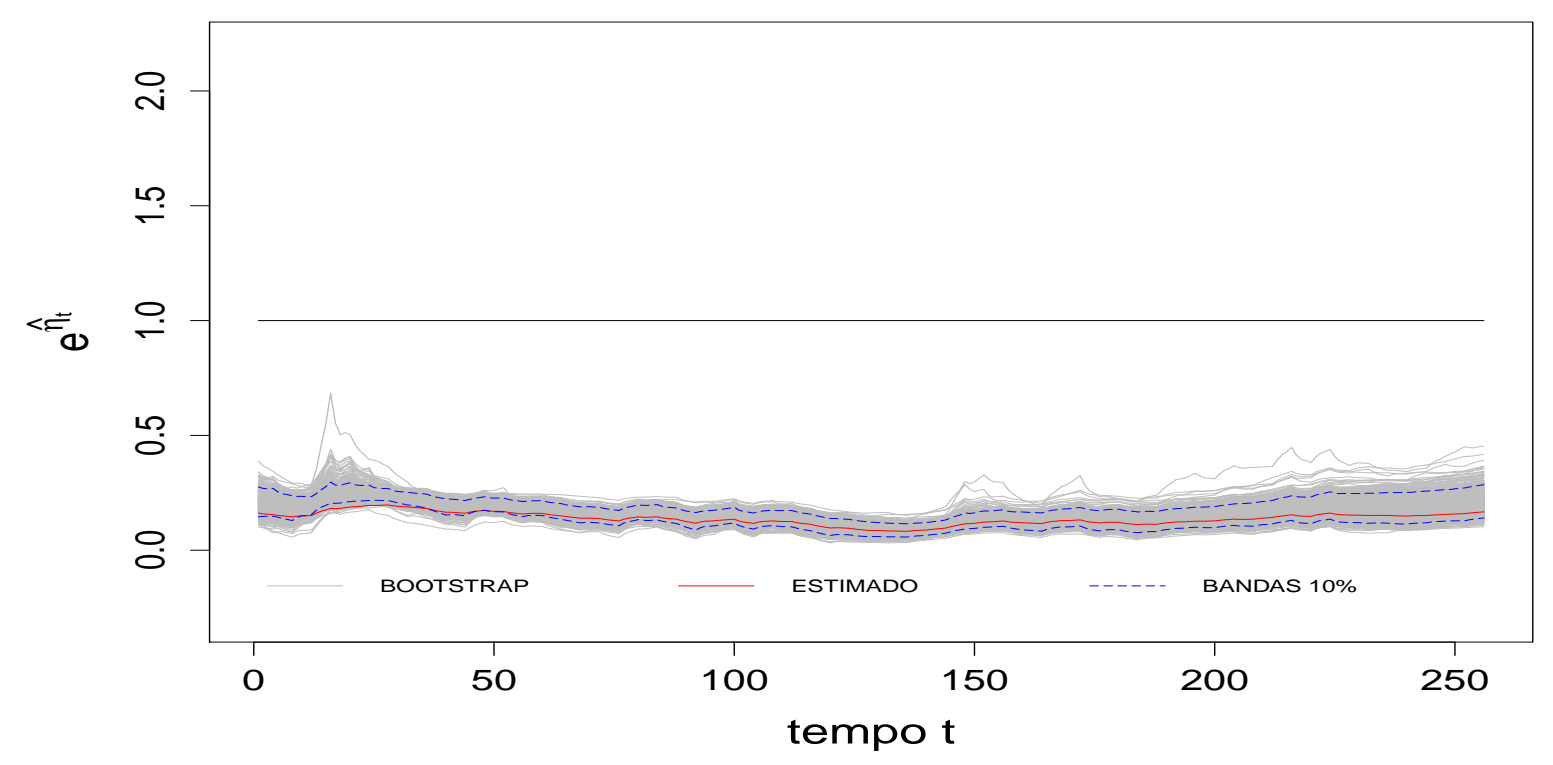

Figura 6.14: Gráficos com quantidade $e^{\eta_{t}}$ estimada, juntamente com bandas de confiança, para os países estrangeiros Bélgica, Canadá, Dinamarca, França.

A Tabela 6.6 contém a combinação de parâmetros utilizada em cada ajuste. Em todos os casos, foi utilizada a família de ondaletas Daubechies. Para testarmos a suposição de normalidade dos resíduos $u_{t}$ dos modelos, apresentamos os valores- $p$ para o teste de normalidade de Shapiro Wilk, cuja hipótese nula $H_{0}$ é de que a série segue uma distribuição normal. Apresentamos também os valores da estatística de razão de verossimilhanças $R V_{C V T}^{(J)}$, para testar a hipótese de intercepto variando com o tempo. Conforme podemos notar pela tabela, existem indícios que nos levam a rejeitar $H_{0}$ em todos os casos.

Tabela 6.6: Modelos de PPC: combinação de parâmetros do modelo, valores- $p$ para o teste de normalidade de Shapiro-Wilk para resíduos do modelo $u_{t}=\left(u_{1 t}, u_{2 t}, u_{3 t}\right)^{\prime}$ e estatística de razão de verossimilhanças $R V_{C V T}^{(J)}$.

\begin{tabular}{|c|c|c|c|c|c|}
\hline & Fam = DaubExPhase & Teste & e Shapir & -Wilk & Teste de $\mathrm{F}$ \\
\hline País est & Combinação de parâmetros & $u_{1 t}$ & $u_{2 t}$ & $u_{3 t}$ & $R V_{C V T}^{(J)}$ \\
\hline & Tiltw & 0.3366 & 0.3932 & 0,0000 & 272.83 \\
\hline França & $\mathrm{J}=5, \mathrm{p}=13$, Filtro $=2$ & 0.4998 & 0.1063 & 0.0015 & 275.86 \\
\hline
\end{tabular}

Com base na interpretação apresentada na Seção 4.7 da quantidade $e^{\eta_{t}}$ podemos considerar que os casos CAN vs. USA e FRA vs. USA devem modelados considerando o intercepto variando com tempo, com $e^{\eta_{t}}$ oscilando entre 0 e 1 . 


\section{Capítulo 7}

\section{Conclusão}

\subsection{Considerações finais}

Nas últimas décadas, o interesse na literatura sobre o tema cointegração aumentou de maneira expressiva. A literatura pertinente a este assunto foi abordada sob inúmeros aspectos e pontos de vista. Trabalhos de revisão como Taylor e Taylor (2004) e suas referências dão conta de como a literatura sobre este tópico é vasta e complexa. O assunto é intensamente debatido e aplicado em livros texto de grande importância na área de séries temporais, como em Hamilton (1994), Baltagi (2008), Tsay (2010), Morettin (2011) e Tsay (2013).

Os modelos tradicionais que tratam deste tópico supõem que o vetor de cointegração não varia ao longo do tempo (Granger, 1986; Johansen, 1988). Entretanto, conforme apontam os trabalhos de Park e Hahn (1999), Bierens e Martins (2010) e Martins (2016), existem evidências de que esta suposição pode ser considerada muito restritiva. Bierens e Martins (2010), por exemplo, utilizaram polinômios de Chebyshev para modelar o vetor de cointegração $\boldsymbol{\beta}_{t}$ variando com o tempo, com a suposição de que $\boldsymbol{\beta}_{t}$ é uma função discreta suave do tempo.

Com base nestas idéias, propomos neste trabalho um modelo de correção de erros vetorial em que também é permitido ao vetor de cointegração variar ao longo do tempo. No entanto, modelamos $\boldsymbol{\beta}_{t}$ por meio de ondaletas. Dadas as características das ondaletas, que podem ser suaves (ou não) (Morettin, 2014), relaxamos a condição imposta em Bierens e Martins (2010) de que $\boldsymbol{\beta}_{t}$ deve ser uma função discreta suave do tempo.

O Capítulo 4 apresenta a construção do modelo de correção de erros vetorial, com o vetor de cointegração decomposto por meio de ondaletas. O capítulo também se dedica à teoria necessária para deduzir os estimadores dos parâmetros do modelo. As maiores contribuições são a apresentação e a construção do modelo, além da dedução dos estimadores não normalizados e normalizados de seus parâmetros. Com base nos resultados teóricos encontrados, foram implementadas rotinas computacionais para definir, estimar 
e apresentar os resultados do modelo. Estas rotinas podem ser visualizadas no Anexo C.

O Capítulo 5 foi dedicado a testar o modelo apresentado no Capítulo 4. Foram propostas diversas situações controladas, em que se verificou a capacidade do modelo de lidar satisfatoriamente com séries cointegradas simuladas, que apresentavam mudança de regime para o vetor de cointegração. Em cada exemplo, foram incluídas uma ou mais mudanças ao longo do tempo. Nos Exemplos 01 e 03, foram consideradas mudanças apenas no nível do vetor de cointegração ao longo do tempo (mudanças lineares abruptas). Nos Exemplos 4 e 5 foram considerados outros tipos de mudanças temporais, persistentes, seguindo decrescimentos linear e logarítmico respectivamente. Neste sentido, o trabalho traz contribuições na área de mudanças de regime, com a apresentação de um modelo capaz de estimar mudanças no vetor de cointegração ao longo do tempo. A metodologia ainda permite testar se as mudanças observadas são estatisticamente significativas.

A utilização de ondaletas agrega versatilidade ao modelo, permitindo o tratamento de mudanças abruptas na relação de cointegração (ao utilizar, por exemplo a base de ondaletas Haar) ou mudanças suaves (ao utilizar, por exemplo, a base de ondaletas Symmlet S8 ou S10). O Capítulo 5 ainda traz o Exemplo 3, em que não foram consideradas mudanças no vetor de cointegração ao longo do tempo. Claramente a intenção foi testar a capacidade do modelo em diferenciar situações com cointegração dinâmica de situações com cointegração usual. O modelo também se mostrou bastante eficiente para estimar os parâmetros simulados neste caso.

O Capítulo 6 foi dedicado a aplicar o modelo apresentado no Capítulo 4 e testado no Capítulo 5. Para isso, foi utilizado o conjunto de dados empregado em Falk e Wang (2003), Bierens e Martins (2010) e Martins (2016). Neste conjunto de dados, trabalhamos com o modelo de PPC variando com o tempo entre os EUA e doze outros países, considerados estrangeiros. Testamos dois casos específicos: modelo sem intercepto e com intercepto.

Para o primeiro caso, apresentado na Seção 6.1, estimamos $\beta_{1 t}$, $\beta_{2 t}$ e $\beta_{3 t}$ considerando a base de ondaletas Daubechies D2, $p=1$ e $J=0,1,2,3,4$ e 5. Os valores das estatísticas $R V_{C V T}^{(J)}$ (e valores-p bootstrap observados) indicam cointegração variando com o tempo entre Estados Unidos e dez dos doze países estrangeiros considerados. Estes resultados estão em concordância com os resultados apresentados em Bierens e Martins (2010). Apenas para Espanha e Itália a relação de PPC não ficou evidente. Foi possível verificar também que utilizar a defasagem $p=1$ empregada em Bierens e Martins (2010) deixa considerável correlação nas séries de resíduos dos modelos ajustados. Logo, foi apresentado um ajuste completo para a relação de cointegração entre Estados Unidos e Canadá, tomando $p=13$. Foram verificados ganhos consideráveis em análise, melhores bandas bootstrap para as componentes do vetor de cointegração e respeito às condições necessárias para que haja cointegração, conforme aponta Martins (2016).

Por fim, apresentamos na Seção 6.2 o caso de PPC considerando o intercepto no modelo. Neste caso, o foco foi estimar a quantidade $e^{\eta_{t}}$ variando com o tempo, já que 
consideramos o vetor $\boldsymbol{\beta}$ como sendo constante. Notamos mais uma vez a versatilidade do modelo em considerar situações ainda mais gerais.

\subsection{Pesquisas futuras}

Martins (2005) apresentou um modelo de correção de erros vetorial onde é permitido ao vetor de cointegração variar com tempo, sendo modelado por meio de Polinômios de Chebychev. Desde então, alguns trabalhos foram publicados pelo autor sobre o tema. Em Bierens e Martins (2010) por exemplo, foram deduzidos vários resultados assintóticos, inclusive para a distribuição da estatística de razão de verossimilhança sob a hipótese nula de cointegração usual. Os autores demonstraram que a estatística segue uma distribuição qui-quadrado com graus de liberdade que dependem de um parâmetro $m$ dos Polinômios de Chebychev. Martins e Gabriel (2013) derivam as condições sob as quais a cointegração variando com o tempo leva a espaços de cointegração que podem ser variantes ou invariantes com o tempo. Além disso, eles esclarecem o papel das restrições de identificação e normalização, demonstrando que as rupturas estruturais nos modelos de correção de erros podem realmente corresponder a relações econômicas estáveis a longo prazo, ao contrário de uma configuração com equação única, na qual uma restrição de identificação é imposta. Martins (2016) por sua vez, revisita o problema da PPC variando com o tempo utilizando simulações de Monte Carlo e Bootstrap, encontrando resultados mais refinados. São quase doze anos de pesquisa com artigos publicados em revistas importantes na área, demonstrando a relevância do assunto, mesmo sobre um tópico tão recorrente na literatura (Taylor e Taylor, 2004).

Seguindo o tabalho destes autores, procuramos mostrar as vantagens de se utilizar as ondaletas como uma alternativa aos Polinômios de Chebychev para modelar o vetor de cointegração variando com o tempo. Procuramos enaltecer a versatilidade do novo modelo e a capacidade de gerarmos conhecimento a partir do mesmo. Sentimo-nos confiantes de que, assim como nos trabalhos citados, a metodologia apresentada aqui tem potencial para gerar novas contribuições para a área de pesquisa.

Neste sentido, algumas questões para futuras pesquisas merecem ser destacadas. Dentre elas, citamos o estudo das propriedades dos estimadores de máxima verossimilhança apresentados no Capítulo 4. Apontamos a necessidade de apresentar as taxas de convergência destes estimadores. Além disso, desejamos aplicar o modelo em séries de dados mais longas, de preferência, englobando o período da crise financeira de 2008. A ideia será testar a capacidade de estimação do modelo frente a conjuntos de dados que apresentem maior volatilidade. Desejamos ainda verificar se a inclusão de variáveis dummy e termos determinísticos melhoram as estimativas dos parâmetros do modelo. 


\section{Apêndice A}

\section{Resultados matriciais}

Proposição A.1. Mínimo de $\left|T^{-1}(Y-B C X)(Y-B C X)^{\prime}\right|$

Sejam $Y$ e $X$ matrizes de ordem $(n \times T)$ de posto $n$ e sejam $A$ e $B$ matrizes com posto $r$ e dimensões $n \times r$ e $r \times n$ repectivamente. Além disso, sejam $\lambda_{1} \geq \lambda_{2} \geq \ldots \geq \lambda_{n}$ os autovalores de

$$
\left(X X^{\prime}\right)^{-\frac{1}{2}} X Y^{\prime}\left(Y Y^{\prime}\right) Y X^{\prime}\left(X X^{\prime}\right)^{\prime-\frac{1}{2}}
$$

com autovetores ortonormais correspondentes $v_{1}, v_{2}, \ldots, v_{n} e\left(X X^{\prime}\right)^{-\frac{1}{2}}$ tal que

$$
\left(X X^{\prime}\right)^{-\frac{1}{2}} X X^{\prime}\left(X X^{\prime}\right)^{\prime-\frac{1}{2}}=I_{n}
$$

Desta forma,

$$
\min _{A, B}\left|T^{-1}(Y-B C X)(Y-B C X)^{\prime}\right|=\left|T^{-1} Y Y^{\prime}\right| \prod_{i=1}^{r}\left(1-\lambda_{i}\right),
$$

com o mínimo sendo atingido para

$$
B=\hat{B}=\left[\begin{array}{llll}
v_{1} & v_{2} & \ldots & v_{r}
\end{array}\right]^{\prime}\left(X X^{\prime}\right)^{-\frac{1}{2}}
$$

$e$

$$
A=\hat{A}=Y X^{\prime} \hat{B}^{\prime}\left(\hat{B} X X^{\prime} \hat{B}^{\prime}\right)^{-1}
$$

\section{Demonstração:}

Este resultado foi demonstrado em Tso (1981).

Conforme aponta Lutkepohl (2005), devemos notar que as matrizes de minimização $\hat{A}$ e $\hat{B}$ não são únicas. Qualquer matriz não singular $F$ de ordem $(r \times r)$ leva a outro conjunto de matrizes de minimização $F \hat{A}$ e $\hat{B} F^{-1}$. 


\section{Apêndice B}

\section{Demonstrações e passagens omissas no texto}

Neste capítulo serão apresentados alguns resultados que foram omitidos do texto, e que podem ser consultados para complementar a leitura do mesmo.

\section{B.1 Demonstrações do Capítulo 2 - Seção 2.3, Equação}

\subsection{7:}

Substituindo a Equação 2.25 na Equação 2.26 e tomando o logaritmo em seguida, temos que

$$
\begin{aligned}
\mathscr{L}(\boldsymbol{\theta}) & =\prod_{t=1}^{T}\left\{\frac{1}{(2 \pi)^{\frac{n}{2}}\left|\boldsymbol{\Sigma}_{u}\right|^{\frac{1}{2}}} \exp \left(-\frac{1}{2}\left(\Delta Y_{t}-\boldsymbol{\alpha} \boldsymbol{\beta}^{\prime} Y_{t-1}-\boldsymbol{\Gamma} \Delta X_{t}\right)^{\prime} \cdot \boldsymbol{\Sigma}_{u}^{-1} \cdot\left(\Delta Y_{t}-\boldsymbol{\alpha} \boldsymbol{\beta}^{\prime} Y_{t-1}-\boldsymbol{\Gamma} \Delta X_{t}\right)\right)\right\} \\
& =\frac{1}{(2 \pi)^{\frac{T n}{2}}\left|\boldsymbol{\Sigma}_{u}\right|^{\frac{T}{2}}} \exp \left\{-\frac{1}{2} \sum_{t=1}^{T}\left(\left(\Delta Y_{t}-\boldsymbol{\alpha} \boldsymbol{\beta}^{\prime} Y_{t-1}-\boldsymbol{\Gamma} \Delta X_{t}\right)^{\prime} \cdot \boldsymbol{\Sigma}_{u}^{-1} \cdot\left(\Delta Y_{t}-\boldsymbol{\alpha} \boldsymbol{\beta}^{\prime} Y_{t-1}-\boldsymbol{\Gamma} \Delta X_{t}\right)\right)\right\} \\
& =\frac{T n}{2} \log 2 \pi-\frac{T}{2} \log \left|\boldsymbol{\Sigma}_{u}\right|-\frac{1}{2} \underbrace{\sum_{t=1}^{T}\left(\left(\Delta Y_{t}-\boldsymbol{\alpha} \boldsymbol{\beta}^{\prime} Y_{t-1}-\boldsymbol{\Gamma} \Delta X_{t}\right)^{\prime} \cdot \boldsymbol{\Sigma}_{u}^{-1} \cdot\left(\Delta Y_{t}-\boldsymbol{\alpha} \boldsymbol{\beta}^{\prime} Y_{t-1}-\boldsymbol{\Gamma} \Delta X_{t}\right)\right)}_{\text {escalar }} .
\end{aligned}
$$

Como o somatório na expressão acima é escalar, o seu valor não muda ao aplicarmos o operador traço $(t r)$, logo

$\mathscr{L}(\boldsymbol{\theta})=\frac{T n}{2} \log 2 \pi-\frac{T}{2} \log \left|\boldsymbol{\Sigma}_{u}\right|-\frac{1}{2} \operatorname{tr}\left\{\sum_{t=1}^{T}\left(\left(\Delta Y_{t}-\boldsymbol{\alpha} \boldsymbol{\beta}^{\prime} Y_{t-1}-\boldsymbol{\Gamma} \Delta X_{t}\right)^{\prime} \cdot \boldsymbol{\Sigma}_{u}^{-1} \cdot\left(\Delta Y_{t}-\boldsymbol{\alpha} \boldsymbol{\beta}^{\prime} Y_{t-1}-\boldsymbol{\Gamma} \Delta X_{t}\right)\right)\right\}$.

Em notação matricial, temos que

$$
\mathscr{L}(\boldsymbol{\theta})=\frac{T n}{2} \log 2 \pi-\frac{T}{2} \log \left|\boldsymbol{\Sigma}_{u}\right|-\frac{1}{2} \operatorname{tr}\left(\left(\Delta Y-\boldsymbol{\alpha} \boldsymbol{\beta}^{\prime} Y_{-1}-\boldsymbol{\Gamma} \Delta X\right)^{\prime} \cdot \boldsymbol{\Sigma}_{u}^{-1} \cdot\left(\Delta Y-\boldsymbol{\alpha} \boldsymbol{\beta}^{\prime} Y_{-1}-\boldsymbol{\Gamma} \Delta X\right)\right) .
$$




\section{B.2 Tabelas longas do Capítulo 5}

\section{B.2.1 Quarto exemplo: Decrescimento linear com Daubechies D2}

Tabela B.1: Estimativas e medidas bootstrap ${ }^{\star}$ sob HA (decrescimento linear para $\beta_{2 t}$ ) continuação da Tabela 5.13.

\begin{tabular}{|c|c|c|c|c|c|}
\hline Parâmetro & Teórico & Estimativa & Intervalo de confiança* & Média* & Erro Padrão* \\
\hline$\vdots$ & $\vdots$ & $\vdots$ & $\vdots$ & $\vdots$ & \\
\hline$d_{40}^{(1,1)}$ & 0,0000 & 0,0000 & {$[0,0000 ; 0,0000]$} & 0,0000 & 0,0000 \\
\hline$d_{40}^{(2,1)}$ & 0,0631 & 0,0556 & {$[0,0275 ; 0,0872]$} & 0,0566 & 0,0149 \\
\hline$d_{41}^{(1,1)}$ & 0,0000 & 0,0000 & {$[0,0000 ; 0,0000]$} & 0,0000 & 0,0000 \\
\hline$d_{41}^{(2,1)}$ & $-0,0008$ & 0,0115 & {$[-0,0076 ; 0,0337]$} & 0,0102 & 0,0301 \\
\hline$d_{42}^{(1,1)}$ & 0,0000 & 0,0000 & {$[0,0000 ; 0,0000]$} & 0,0000 & 0,0000 \\
\hline$d_{42}^{(2,1)}$ & 0,0000 & $-0,0079$ & {$[-0,0233 ; 0,0100]$} & $-0,0075$ & 0,0090 \\
\hline$d_{43}^{(1,1)}$ & 0,0000 & 0,0000 & {$[0,0000 ; 0,0000]$} & 0,0000 & 0,0000 \\
\hline$d_{43}^{(2,1)}$ & 0,0000 & $-0,0044$ & {$[-0,0227 ; 0,0171]$} & $-0,0043$ & 0,0089 \\
\hline$d_{44}^{(1,1)}$ & 0,0000 & 0,0000 & {$[0,0000 ; 0,0000]$} & 0,0000 & 0,0000 \\
\hline$d_{44}^{(2,1)}$ & 0,0000 & $-0,0002$ & {$[-0,0191 ; 0,0195]$} & $-0,0001$ & 0,0089 \\
\hline$d_{45}^{(1,1)}$ & 0,0000 & 0,0000 & {$[0,0000 ; 0,0000]$} & 0,0000 & 0,0000 \\
\hline$d_{45}^{(2,1)}$ & 0,0000 & 0,0158 & {$[-0,0008 ; 0,0310]$} & 0,0150 & 0,0086 \\
\hline$d_{46}^{(1,1)}$ & 0,0000 & 0,0000 & {$[0,0000 ; 0,0000]$} & 0,0000 & 0,0000 \\
\hline$d_{46}^{(2,1)}$ & 0,0000 & $-0,0091$ & {$[-0,0239 ; 0,0053]$} & $-0,0091$ & 0,0085 \\
\hline$d_{47}^{(1,1)}$ & 0,0000 & 0,0000 & {$[0,0000 ; 0,0000]$} & 0,0000 & 0,0000 \\
\hline$d_{47}^{(2,1)}$ & 0,0000 & $-0,0026$ & {$[-0,0175 ; 0,0155]$} & $-0,0023$ & 0,0081 \\
\hline$d_{48}^{(1,1)}$ & 0,0000 & 0,0000 & {$[0,0000 ; 0,0000]$} & 0,0000 & 0,0000 \\
\hline$d_{48}^{(2,1)}$ & 0,0000 & 0,0094 & {$[-0,0035 ; 0,0241]$} & 0,0096 & 0,0058 \\
\hline$d_{49}^{(1,1)}$ & 0,0000 & 0,0000 & {$[0,0000 ; 0,0000]$} & 0,0000 & 0,0000 \\
\hline$d_{49}^{(2,1)}$ & 0,0000 & $-0,0007$ & {$[-0,0118 ; 0,0109$} & $-0,0007$ & 0,0077 \\
\hline$d_{410}^{(1,1)}$ & 0,0000 & 0,0000 & {$[0,0000 ; 0,0000]$} & 0,0000 & 0,0000 \\
\hline$d_{410}^{(2,1)}$ & 0,0000 & $-0,0023$ & {$[-0,0196 ; 0,0087]$} & $-0,0026$ & 0,0060 \\
\hline$d_{411}^{(1,1)}$ & 0,0000 & 0,0000 & {$[0,0000 ; 0,0000]$} & 0,0000 & 0,0000 \\
\hline$d_{411}^{(2,1)}$ & 0,0000 & $-0,0012$ & {$[-0,0118 ; 0,0109$} & $-0,0009$ & 0,0054 \\
\hline$d_{412}^{(1,1)}$ & 0,0000 & 0,0000 & {$[0,0000 ; 0,0000]$} & 0,0000 & 0,0000 \\
\hline$d_{412}^{(2,1)}$ & 0,0000 & 0,0041 & {$[-0,0076 ; 0,0168]$} & 0,0039 & 0,0053 \\
\hline$d_{413}^{(1,1)}$ & 0,0000 & 0,0000 & {$[0,0000 ; 0,0000]$} & 0,0000 & 0,0000 \\
\hline$d_{413}^{(2,1)}$ & 0,0000 & $-0,0013$ & {$[-0,0109 ; 0,0116]$} & $-0,0009$ & 0,0052 \\
\hline$d_{414}^{(1,1)}$ & 0,0000 & 0,0000 & {$[0,0000 ; 0,0000]$} & 0,0000 & 0,0000 \\
\hline
\end{tabular}

(Continua na página seguinte) 
Tabela B.1 - Continuação da página anterior

\begin{tabular}{|c|c|c|c|c|c|}
\hline Parâmetro & Teórico & Estimativa & Intervalo de confiança* & Média* & Erro Padrão* \\
\hline$d_{414}^{(2,1)}$ & 0,0000 & $-0,0013$ & {$[-0,0165 ; 0,0133]$} & $-0,0013$ & 0,0064 \\
\hline$d_{415}^{(1,1)}$ & 0,0000 & 0,0000 & {$[0,0000 ; 0,0000]$} & 0,0000 & 0,0000 \\
\hline$d_{415}^{(2,1)}$ & $-0,0082$ & $-0,0063$ & {$[-0,0191 ; 0,0060]$} & $-0,0064$ & 0,0053 \\
\hline$d_{50}^{(1,1)}$ & 0,0000 & 0,0000 & {$[0,0000 ; 0,0000$} & 0,0000 & 0,0000 \\
\hline$d_{50}^{(2,1)}$ & 0,0637 & 0,0445 & {$[0,0160 ; 0,0822$} & 0,0464 & 0,0182 \\
\hline$d_{51}^{(1,1)}$ & 0,0000 & 0,0000 & {$[0,0000 ; 0,0000$} & 0,0000 & 0,0000 \\
\hline$d_{51}^{(2,1)}$ & $-0,0001$ & 0,0087 & {$[-0,0207 ; 0,0328$} & 0,0080 & 0,0142 \\
\hline$d_{52}^{(1,1)}$ & 0,0000 & 0,0000 & {$[0,0000 ; 0,0000$} & 0,0000 & 0,0000 \\
\hline$d_{52}^{(2,1)}$ & 0,0000 & $-0,0052$ & {$[-0,0281 ; 0,0165$} & $-0,0050$ & 0,0220 \\
\hline$d_{53}^{(1,1)}$ & 0,0000 & 0,0000 & {$[0,0000 ; 0,0000]$} & 0,0000 & 0,0000 \\
\hline$d_{53}^{(2,1)}$ & 0,0000 & $-0,0032$ & {$[-0,0226 ; 0,0196$} & $-0,0017$ & 0,0182 \\
\hline$d_{54}^{(1,1)}$ & 0,0000 & 0,0000 & {$[0,0000 ; 0,0000$} & 0,0000 & 0,0000 \\
\hline$d_{54}^{(2,1)}$ & 0,0000 & 0,0015 & {$[-0,0171 ; 0,0176$} & 0,0010 & 0,0081 \\
\hline$d_{55}^{(1,1)}$ & 0,0000 & 0,0000 & {$[0,0000 ; 0,0000]$} & 0,0000 & 0,0000 \\
\hline$d_{55}^{(2,1)}$ & 0,0000 & 0,0166 & {$[-0,0013 ; 0,0352$} & 0,0161 & 0,0090 \\
\hline$d_{56}^{(1,1)}$ & 0,0000 & 0,0000 & {$[0,0000 ; 0,0000$} & 0,0000 & 0,0000 \\
\hline$d_{56}^{(2,1)}$ & 0,0000 & $-0,0065$ & {$[-0,0217 ; 0,0145]$} & $-0,0057$ & 0,0082 \\
\hline$d_{57}^{(1,1)}$ & 0,0000 & 0,0000 & {$[0,0000 ; 0,0000]$} & 0,0000 & 0,0000 \\
\hline$d_{57}^{(2,1)}$ & 0,0000 & $-0,0052$ & {$[-0,0208 ; 0,0175]$} & $-0,0044$ & 0,0086 \\
\hline$d_{58}^{(1,1)}$ & 0,0000 & 0,0000 & {$[0,0000 ; 0,0000$} & 0,0000 & 0,0000 \\
\hline$d_{58}^{(2,1)}$ & 0,0000 & 0,0089 & {$[-0,0075 ; 0,0236$} & 0,0090 & 0,0072 \\
\hline$d_{59}^{(1,1)}$ & 0,0000 & 0,0000 & {$[0,0000 ; 0,0000$} & 0,0000 & 0,0000 \\
\hline$d_{59}^{(2,1)}$ & 0,0000 & $-0,0047$ & {$[-0,0262 ; 0,0124]$} & $-0,0046$ & 0,0085 \\
\hline$d_{510}^{(1,1)}$ & 0,0000 & 0,0000 & {$[0,0000 ; 0,0000$} & 0,0000 & 0,0000 \\
\hline$d_{510}^{(2,1)}$ & 0,0000 & $-0,0061$ & {$[-0,0190 ; 0,0152$} & $-0,0050$ & 0,0094 \\
\hline$d_{511}^{(1,1)}$ & 0,0000 & 0,0000 & {$[0,0000 ; 0,0000$} & 0,0000 & 0,0000 \\
\hline$d_{511}^{(2,1)}$ & 0,0000 & $-0,0131$ & {$[-0,0260 ; 0,0034$} & $-0,0122$ & 0,0069 \\
\hline$d_{512}^{(1,1)}$ & 0,0000 & 0,0000 & {$[0,0000 ; 0,0000$} & 0,0000 & 0,0000 \\
\hline$d_{512}^{(2,1)}$ & 0,0000 & $-0,0072$ & {$[-0,0236 ; 0,0073$} & $-0,0070$ & 0,0080 \\
\hline$d_{513}^{(1,1)}$ & 0,0000 & 0,0000 & {$[0,0000 ; 0,0000$} & 0,0000 & 0,0000 \\
\hline$d_{513}^{(2,1)}$ & 0,0000 & 0,0008 & {$[-0,0157 ; 0,0190$} & 0,0013 & 0,0087 \\
\hline$d_{514}^{(1,1)}$ & 0,0000 & 0,0000 & {$[0,0000 ; 0,0000$} & 0,0000 & 0,0000 \\
\hline$d_{514}^{(2,1)}$ & 0,0000 & 0,0008 & {$[-0,0149 ; 0,0123$} & 0,0008 & 0,0065 \\
\hline$d_{515}^{(1,1)}$ & 0,0000 & 0,0000 & {$[0,0000 ; 0,0000$} & 0,0000 & 0,0000 \\
\hline$d_{515}^{(2,1)}$ & 0,0000 & 0,0057 & {$[-0,0091 ; 0,0201]$} & 0,0055 & 0,0085 \\
\hline$d_{516}^{(1,1)}$ & 0,0000 & 0,0000 & {$[0,0000 ; 0,0000]$} & 0,0000 & 0,0000 \\
\hline
\end{tabular}

(Continua na página seguinte) 
Tabela B.1 - Continuação da página anterior

\begin{tabular}{|c|c|c|c|c|c|}
\hline Parâmetro & Teórico & Estimativa & Intervalo de confiança* & Média* & Erro Padrão* \\
\hline$d_{516}^{(2,1)}$ & 0,0000 & 0,0087 & {$[-0,0041 ; 0,0184$} & 0,0088 & 0,0053 \\
\hline$d_{517}^{(1,1)}$ & 0,0000 & 0,0000 & {$[0,0000 ; 0,0000]$} & 0,0000 & 0,0000 \\
\hline$d_{517}^{(2,1)}$ & 0,0000 & 0,0035 & {$[-0,0153 ; 0,0164]$} & 0,0038 & 0,0069 \\
\hline$d_{518}^{(1,1)}$ & 0,0000 & 0,0000 & {$[0,0000 ; 0,0000]$} & 0,0000 & 0,0000 \\
\hline$d_{518}^{(2,1)}$ & 0,0000 & 0,0080 & {$[-0,0054 ; 0,0176]$} & 0,0076 & 0,0059 \\
\hline$d_{519}^{(1,1)}$ & 0,0000 & 0,0000 & {$[0,0000 ; 0,0000]$} & 0,0000 & 0,0000 \\
\hline$d_{519}^{(2,1)}$ & 0,0000 & $-0,0060$ & {$[-0,0162 ; 0,0105]$} & $-0,0055$ & 0,0080 \\
\hline$d_{520}^{(1,1)}$ & 0,0000 & 0,0000 & {$[0,0000 ; 0,0000]$} & 0,0000 & 0,0000 \\
\hline$d_{520}^{(2,1)}$ & 0,0000 & 0,0014 & {$[-0,0147 ; 0,0137$} & 0,0015 & 0,0061 \\
\hline$d_{521}^{(1,1)}$ & 0,0000 & 0,0000 & {$[0,0000 ; 0,0000]$} & 0,0000 & 0,0000 \\
\hline$d_{521}^{(2,1)}$ & 0,0000 & 0,0017 & {$[-0,0107 ; 0,0134]$} & 0,0014 & 0,0064 \\
\hline$d_{522}^{(1,1)}$ & 0,0000 & 0,0000 & {$[0,0000 ; 0,0000]$} & 0,0000 & 0,0000 \\
\hline$d_{522}^{(2,1)}$ & 0,0000 & $-0,0017$ & {$[-0,0110 ; 0,0084]$} & $-0,0018$ & 0,0047 \\
\hline$d_{523}^{(1,1)}$ & 0,0000 & 0,0000 & {$[0,0000 ; 0,0000]$} & 0,0000 & 0,0000 \\
\hline$d_{523}^{(2,1)}$ & 0,0000 & $-0,0025$ & {$[-0,0150 ; 0,0096$} & $-0,0026$ & 0,0058 \\
\hline$d_{524}^{(1,1)}$ & 0,0000 & 0,0000 & {$[0,0000 ; 0,0000$} & 0,0000 & 0,0000 \\
\hline$d_{524}^{(2,1)}$ & 0,0000 & $-0,0018$ & {$[-0,0123 ; 0,0149$} & $-0,0010$ & 0,0076 \\
\hline$d_{525}^{(1,1)}$ & 0,0000 & 0,0000 & {$[0,0000 ; 0,0000$} & 0,0000 & 0,0000 \\
\hline$d_{525}^{(2,1)}$ & 0,0000 & 0,0019 & {$[-0,0071 ; 0,0125$} & 0,0020 & 0,0063 \\
\hline$d_{526}^{(1,1)}$ & 0,0000 & 0,0000 & {$[0,0000 ; 0,0000$} & 0,0000 & 0,0000 \\
\hline$d_{526}^{(2,1)}$ & 0,0000 & 0,0006 & {$[-0,0126 ; 0,0080$} & 0,0001 & 0,0049 \\
\hline$d_{527}^{(1,1)}$ & 0,0000 & 0,0000 & {$[0,0000 ; 0,0000$} & 0,0000 & 0,0000 \\
\hline$d_{527}^{(2,1)}$ & 0,0000 & $-0,0001$ & {$[-0,0145 ; 0,0100$} & 0,0001 & 0,0055 \\
\hline$d_{528}^{(1,1)}$ & 0,0000 & 0,0000 & {$[0,0000 ; 0,0000$} & 0,0000 & 0,0000 \\
\hline$d_{528}^{(2,1)}$ & 0,0000 & $-0,0016$ & {$[-0,0142 ; 0,0113$} & $-0,0013$ & 0,0110 \\
\hline$d_{529}^{(1,1)}$ & 0,0000 & 0,0000 & {$[0,0000 ; 0,0000$} & 0,0000 & 0,0000 \\
\hline$d_{529}^{(2,1)}$ & 0,0000 & 0,0005 & {$[-0,0092 ; 0,0149$} & 0,0012 & 0,0060 \\
\hline$d_{530}^{(1,1)}$ & 0,0000 & 0,0000 & {$[0,0000 ; 0,0000$} & 0,0000 & 0,0000 \\
\hline$d_{530}^{(2,1)}$ & 0,0000 & 0,0010 & {$[-0,0121 ; 0,0108$} & 0,0009 & 0,0050 \\
\hline$d_{531}^{(1,1)}$ & 0,0000 & 0,0000 & {$[0,0000 ; 0,0000$} & 0,0000 & 0,0000 \\
\hline$d_{531}^{(2,1)}$ & 0,0000 & $-0,0012$ & {$[-0,0147 ; 0,0110$} & $-0,0014$ & 0,0082 \\
\hline
\end{tabular}




\section{B.2.2 Sexto exemplo: Decrescimento logarítmico com Symmlet $S 8$ (considerando defasagem do modelo)}

Tabela B.2: Estimativas e medidas bootstrap ${ }^{\star}$ sob HA (decrescimento logarítmico para $\beta_{2 t}$ ) - continuação da Tabela 5.20.

\begin{tabular}{|c|c|c|c|c|c|}
\hline Parâmetro & Teórico & Estimativa & Intervalo de confiança* & Média* & Erro Padrão* \\
\hline$\vdots$ & $\vdots$ & $\vdots$ & $\vdots$ & $\vdots$ & \\
\hline$d_{40}^{(1,1)}$ & 0,0000 & 0,0000 & {$[0,0000 ; 0,0000]$} & 0,0000 & 0,0000 \\
\hline$d_{40}^{(2,1)}$ & $-0,0735$ & $-0,0553$ & {$[-0,0708 ;-0,0381]$} & $-0,0543$ & 0,0082 \\
\hline$d_{41}^{(1,1)}$ & 0,0000 & 0,0000 & {$[0,0000 ; 0,0000]$} & 0,0000 & 0,0000 \\
\hline$d_{41}^{(2,1)}$ & 0,0153 & 0,0176 & {$[0,0056 ; 0,0311]$} & 0,0179 & 0,0058 \\
\hline$d_{42}^{(1,1)}$ & 0,0000 & 0,0000 & {$[0,0000 ; 0,0000]$} & 0,0000 & 0,0000 \\
\hline$d_{42}^{(2,1)}$ & $-0,0039$ & $-0,0071$ & {$[-0,0152 ; 0,0039]$} & $-0,0067$ & 0,0048 \\
\hline$d_{43}^{(1,1)}$ & 0,0000 & 0,0000 & {$[0,0000 ; 0,0000]$} & 0,0000 & 0,0000 \\
\hline$d_{43}^{(2,1)}$ & 0,0004 & 0,0029 & {$[-0,0076 ; 0,0119]$} & 0,0027 & 0,0049 \\
\hline$d_{44}^{(1,1)}$ & 0,0000 & 0,0000 & {$[0,0000 ; 0,0000]$} & 0,0000 & 0,0000 \\
\hline$d_{44}^{(2,1)}$ & 0,0000 & $-0,0031$ & {$[-0,0131 ; 0,0041]$} & $-0,0034$ & 0,0040 \\
\hline$d_{45}^{(1,1)}$ & 0,0000 & 0,0000 & {$[0,0000 ; 0,0000]$} & 0,0000 & 0,0000 \\
\hline$d_{45}^{(2,1)}$ & 0,0000 & $-0,0010$ & {$[-0,0120 ; 0,0114]$} & $-0,0007$ & 0,0051 \\
\hline$d_{46}^{(1,1)}$ & 0,0000 & 0,0000 & {$[0,0000 ; 0,0000]$} & 0,0000 & 0,0000 \\
\hline$d_{46}^{(2,1)}$ & 0,0000 & 0,0061 & {$[-0,0020 ; 0,0166]$} & 0,0061 & 0,0038 \\
\hline$d_{47}^{(1,1)}$ & 0,0000 & 0,0000 & {$[0,0000 ; 0,0000]$} & 0,0000 & 0,0000 \\
\hline$d_{47}^{(2,1)}$ & 0,0000 & 0,0052 & {$[-0,0041 ; 0,0165]$} & 0,0053 & 0,0041 \\
\hline$d_{48}^{(1,1)}$ & 0,0000 & 0,0000 & {$[0,0000 ; 0,0000]$} & 0,0000 & 0,0000 \\
\hline$d_{48}^{(2,1)}$ & 0,0000 & $-0,0031$ & {$[-0,0137 ; 0,0052]$} & $-0,0031$ & 0,0042 \\
\hline$d_{49}^{(1,1)}$ & 0,0000 & 0,0000 & {$[0,0000 ; 0,0000]$} & 0,0000 & 0,0000 \\
\hline$d_{49}^{(2,1)}$ & 0,0000 & $-0,0028$ & {$[-0,0121 ; 0,0053]$} & $-0,0028$ & 0,0037 \\
\hline$d_{410}^{(1,1)}$ & 0,0000 & 0,0000 & {$[0,0000 ; 0,0000]$} & 0,0000 & 0,0000 \\
\hline$d_{410}^{(2,1)}$ & 0,0000 & 0,0016 & {$[-0,0068 ; 0,0065]$} & 0,0012 & 0,0034 \\
\hline$d_{411}^{(1,1)}$ & 0,0000 & 0,0000 & {$[0,0000 ; 0,0000]$} & 0,0000 & 0,0000 \\
\hline$d_{411}^{(2,1)}$ & 0,0000 & 0,0012 & {$[-0,0063 ; 0,0092]$} & 0,0012 & 0,0032 \\
\hline$d_{412}^{(1,1)}$ & 0,0000 & 0,0000 & {$[0,0000 ; 0,0000]$} & 0,0000 & 0,0000 \\
\hline$d_{412}^{(2,1)}$ & $-0,0005$ & $-0,0024$ & {$[-0,0102 ; 0,0054]$} & $-0,0023$ & 0,0036 \\
\hline$d_{413}^{(1,1)}$ & 0,0000 & 0,0000 & {$[0,0000 ; 0,0000]$} & 0,0000 & 0,0000 \\
\hline$d_{413}^{(2,1)}$ & 0,0032 & 0,0022 & {$[-0,0077 ; 0,0081]$} & 0,0018 & 0,0034 \\
\hline$d_{414}^{(1,1)}$ & 0,0000 & 0,0000 & {$[0,0000 ; 0,0000]$} & 0,0000 & 0,0000 \\
\hline$d_{414}^{(2,1)}$ & $-0,0065$ & $-0,0032$ & {$[-0,0103 ; 0,0044]$} & $-0,0030$ & 0,0034 \\
\hline$d_{415}^{(1,1)}$ & 0,0000 & 0,0000 & {$[0,0000 ; 0,0000]$} & 0,0000 & 0,0000 \\
\hline
\end{tabular}

(Continua na página seguinte) 
Tabela B.2 - Continuação da página anterior

\begin{tabular}{|c|c|c|c|c|c|}
\hline Parâmetro & Teórico & Estimativa & Intervalo de confiança* & Média* & Erro Padrão* \\
\hline$d_{415}^{(2,1)}$ & 0,0232 & $-0,0049$ & {$[-0,0154 ; 0,0012]$} & $-0,0070$ & 0,0039 \\
\hline$d_{50}^{(1,1)}$ & 0,0000 & 0,0000 & {$[0,0000 ; 0,0000]$} & 0,0000 & 0,0000 \\
\hline$d_{50}^{(2,1)}$ & $-0,0649$ & 0,0114 & {$[-0,0058 ; 0,0313]$} & 0,0162 & 0,0093 \\
\hline$d_{51}^{(1,1)}$ & 0,0000 & 0,0000 & {$[0,0000 ; 0,0000]$} & 0,0000 & 0,0000 \\
\hline$d_{51}^{(2,1)}$ & 0,0169 & 0,0041 & {$[-0,0105 ; 0,0268]$} & 0,0045 & 0,0085 \\
\hline$d_{52}^{(1,1)}$ & 0,0000 & 0,0000 & {$[0,0000 ; 0,0000]$} & 0,0000 & 0,0000 \\
\hline$d_{52}^{(2,1)}$ & $-0,0041$ & $-0,0013$ & {$[-0,0134 ; 0,0097]$} & $-0,0012$ & 0,0050 \\
\hline$d_{53}^{(1,1)}$ & 0,0000 & 0,0000 & {$[0,0000 ; 0,0000]$} & 0,0000 & 0,0000 \\
\hline$d_{53}^{(2,1)}$ & 0,0004 & 0,0024 & {$[-0,0069 ; 0,0119]$} & 0,0024 & 0,0043 \\
\hline$d_{54}^{(1,1)}$ & 0,0000 & 0,0000 & {$[0,0000 ; 0,0000]$} & 0,0000 & 0,0000 \\
\hline$d_{54}^{(2,1)}$ & 0,0000 & $-0,0006$ & {$[-0,0108 ; 0,0062]$} & $-0,0011$ & 0,0045 \\
\hline$d_{55}^{(1,1)}$ & 0,0000 & 0,0000 & {$[0,0000 ; 0,0000]$} & 0,0000 & 0,0000 \\
\hline$d_{55}^{(2,1)}$ & 0,0000 & $-0,0040$ & {$[-0,0144 ; 0,0072]$} & $-0,0035$ & 0,0052 \\
\hline$d_{56}^{(1,1)}$ & 0,0000 & 0,0000 & {$[0,0000 ; 0,0000]$} & 0,0000 & 0,0000 \\
\hline$d_{56}^{(2,1)}$ & 0,0000 & 0,0021 & {$[-0,0088 ; 0,0104]$} & 0,0019 & 0,0050 \\
\hline$d_{57}^{(1,1)}$ & 0,0000 & 0,0000 & {$[0,0000 ; 0,0000]$} & 0,0000 & 0,0000 \\
\hline$d_{57}^{(2,1)}$ & 0,0000 & $-0,0021$ & {$[-0,0119 ; 0,0063]$} & $-0,0023$ & 0,0042 \\
\hline$d_{58}^{(1,1)}$ & 0,0000 & 0,0000 & {$[0,0000 ; 0,0000]$} & 0,0000 & 0,0000 \\
\hline$d_{58}^{(2,1)}$ & 0,0000 & $-0,0051$ & {$[-0,0146 ; 0,0023$} & $-0,0052$ & 0,0037 \\
\hline$d_{59}^{(1,1)}$ & 0,0000 & 0,0000 & {$[0,0000 ; 0,0000]$} & 0,0000 & 0,0000 \\
\hline$d_{59}^{(2,1)}$ & 0,0000 & 0,0013 & {$[-0,0082 ; 0,0087]$} & 0,0010 & 0,0040 \\
\hline$d_{510}^{(1,1)}$ & 0,0000 & 0,0000 & {$[0,0000 ; 0,0000]$} & 0,0000 & 0,0000 \\
\hline$d_{510}^{(2,1)}$ & 0,0000 & $-0,0012$ & {$[-0,0104 ; 0,0093$} & $-0,0009$ & 0,0042 \\
\hline$d_{511}^{(1,1)}$ & 0,0000 & 0,0000 & {$[0,0000 ; 0,0000]$} & 0,0000 & 0,0000 \\
\hline$d_{511}^{(2,1)}$ & 0,0000 & 0,0050 & {$[-0,0046 ; 0,0117]$} & 0,0049 & 0,0049 \\
\hline$d_{512}^{(1,1)}$ & 0,0000 & 0,0000 & {$[0,0000 ; 0,0000]$} & 0,0000 & 0,0000 \\
\hline$d_{512}^{(2,1)}$ & 0,0000 & 0,0067 & {$[-0,0015 ; 0,0123]$} & 0,0066 & 0,0037 \\
\hline$d_{513}^{(1,1)}$ & 0,0000 & 0,0000 & {$[0,0000 ; 0,0000]$} & 0,0000 & 0,0000 \\
\hline$d_{513}^{(2,1)}$ & 0,0000 & 0,0004 & {$[-0,0078 ; 0,0075]$} & 0,0004 & 0,0041 \\
\hline$d_{514}^{(1,1)}$ & 0,0000 & 0,0000 & {$[0,0000 ; 0,0000]$} & 0,0000 & 0,0000 \\
\hline$d_{514}^{(2,1)}$ & 0,0000 & 0,0029 & {$[-0,0030 ; 0,0110]$} & 0,0029 & 0,0036 \\
\hline$d_{515}^{(1,1)}$ & 0,0000 & 0,0000 & {$[0,0000 ; 0,0000]$} & 0,0000 & 0,0000 \\
\hline$d_{515}^{(2,1)}$ & 0,0000 & $-0,0020$ & {$[-0,0096 ; 0,0086]$} & $-0,0017$ & 0,0037 \\
\hline$d_{516}^{(1,1)}$ & 0,0000 & 0,0000 & {$[0,0000 ; 0,0000]$} & 0,0000 & 0,0000 \\
\hline$d_{516}^{(2,1)}$ & 0,0000 & $-0,0011$ & {$[-0,0087 ; 0,0066]$} & $-0,0011$ & 0,0044 \\
\hline$d_{517}^{(1,1)}$ & 0,0000 & 0,0000 & {$[0,0000 ; 0,0000]$} & 0,0000 & 0,0000 \\
\hline
\end{tabular}

(Continua na página seguinte) 
Tabela B.2 - Continuação da página anterior

\begin{tabular}{|c|c|c|c|c|c|}
\hline Parâmetro & Teórico & Estimativa & Intervalo de confiança* & Média* & Erro Padrão* \\
\hline$d_{517}^{(2,1)}$ & 0,0000 & $-0,0025$ & {$[-0,0104 ; 0,0052$} & $-0,0025$ & 0,0039 \\
\hline$d_{518}^{(1,1)}$ & 0,0000 & 0,0000 & {$[0,0000 ; 0,0000$} & 0,0000 & 0,0000 \\
\hline$d_{518}^{(2,1)}$ & 0,0000 & $-0,0071$ & {$[-0,0133 ; 0,0000$} & $-0,0070$ & 0,0032 \\
\hline$d_{519}^{(1,1)}$ & 0,0000 & 0,0000 & {$[0,0000 ; 0,0000$} & 0,0000 & 0,0000 \\
\hline$d_{519}^{(2,1)}$ & 0,0000 & 0,0025 & {$[-0,0047 ; 0,0105$} & 0,0023 & 0,0044 \\
\hline$d_{520}^{(1,1)}$ & 0,0000 & 0,0000 & {$[0,0000 ; 0,0000$} & 0,0000 & 0,0000 \\
\hline$d_{520}^{(2,1)}$ & 0,0000 & $-0,0009$ & {$[-0,0089 ; 0,0069$} & $-0,0010$ & 0,0037 \\
\hline$d_{521}^{(1,1)}$ & 0,0000 & 0,0000 & {$[0,0000 ; 0,0000$} & 0,0000 & 0,0000 \\
\hline$d_{521}^{(2,1)}$ & 0,0000 & $-0,0007$ & {$[-0,0065 ; 0,0059$} & $-0,0005$ & 0,0037 \\
\hline$d_{522}^{(1,1)}$ & 0,0000 & 0,0000 & {$[0,0000 ; 0,0000$} & 0,0000 & 0,0000 \\
\hline$d_{522}^{(2,1)}$ & 0,0000 & 0,0004 & {$[-0,0071 ; 0,0079$} & 0,0004 & 0,0030 \\
\hline$d_{523}^{(1,1)}$ & 0,0000 & 0,0000 & {$[0,0000 ; 0,0000$} & 0,0000 & 0,0000 \\
\hline$d_{523}^{(2,1)}$ & 0,0000 & 0,0007 & {$[-0,0062 ; 0,0069$} & 0,0007 & 0,0037 \\
\hline$d_{524}^{(1,1)}$ & 0,0000 & 0,0000 & {$[0,0000 ; 0,0000$} & 0,0000 & 0,0000 \\
\hline$d_{524}^{(2,1)}$ & 0,0000 & 0,0012 & {$[-0,00780,0065]$} & 0,0009 & 0,0035 \\
\hline$d_{525}^{(1,1)}$ & 0,0000 & 0,0000 & {$[0,00000,0000$} & 0,0000 & 0,0000 \\
\hline$d_{525}^{(2,1)}$ & 0,0000 & $-0,0001$ & {$[-0,00640,0069]$} & 0,0000 & 0,0028 \\
\hline$d_{526}^{(1,1)}$ & 0,0000 & 0,0000 & {$[0,00000,0000]$} & 0,0000 & 0,0000 \\
\hline$d_{526}^{(2,1)}$ & 0,0000 & 0,0004 & {$[-0,00570,0078]$} & 0,0006 & 0,0029 \\
\hline$d_{527}^{(1,1)}$ & 0,0000 & 0,0000 & {$[0,00000,0000$} & 0,0000 & 0,0000 \\
\hline$d_{527}^{(2,1)}$ & 0,0000 & 0,0004 & {$[-0,00660,0054]$} & 0,0003 & 0,0035 \\
\hline$d_{528}^{(1,1)}$ & 0,0000 & 0,0000 & {$[0,00000,0000]$} & 0,0000 & 0,0000 \\
\hline$d_{528}^{(2,1)}$ & $-0,0003$ & 0,0004 & {$[-0,00460,0055]$} & 0,0005 & 0,0027 \\
\hline$d_{529}^{(1,1)}$ & 0,0000 & 0,0000 & {$[0,00000,0000$} & 0,0000 & 0,0000 \\
\hline$d_{529}^{(2,1)}$ & 0,0017 & $-0,0002$ & {$[-0,00520,0058]$} & $-0,0001$ & 0,0036 \\
\hline$d_{530}^{(1,1)}$ & 0,0000 & 0,0000 & {$[0,00000,0000$} & 0,0000 & 0,0000 \\
\hline$d_{530}^{(2,1)}$ & $-0,0032$ & 0,0000 & {$[-0,00430,0056]$} & 0,0004 & 0,0028 \\
\hline$d_{531}^{(1,1)}$ & 0,0000 & 0,0000 & {$[0,00000,0000]$} & 0,0000 & 0,0000 \\
\hline$d_{531}^{(2,1)}$ & $-0,0025$ & $-0,0074$ & {$[-0,0145-0,0036]$} & $-0,0082$ & 0,0027 \\
\hline
\end{tabular}




\section{Apêndice C}

\section{Elementos computacionais utilizados no trabalho}

\section{C.1 O software $R$ e o pacote wavethresh}

O software R é simultaneamente linguagem e ambiente para computação estatística e gráfica de alta performance. O software fornece uma ampla variedade de técnicas estatísticas (modelagem linear e não-linear, testes estatísticos clássicos, análise de séries temporais, classificação, análise de cluster, etc). Existem diversos softwares pagos que geralmente são usados como veículos para a disseminação de métodos de pesquisa em Estatística. No entanto, a linguagem $\mathrm{R}$ aparece como uma alternativa Open Source (gratuita) para o mesmo propósito. (R-project, 2016).

Como o software $\mathrm{R}$ foi construído em torno de uma linguagem computacional, ele permite aos usuários adicionar funcionalidades por meio da construção de novas funções e pacotes. Existem inúmeros pacotes especializados em lidar com diversas áreas de pesquisa em Estatística. Estes pacotes podem ser acrescentados via linhas de comando digitadas diretamente no programa. Dependendo do interesse, o pesquisador pode buscar os pacotes com funções de interesse previamente implementadas por outros pesquisadores.

No presente trabalho, utilizamos pacotes com funções para o cálculo matricial (geigen, car) e para o tratamento de séries temporais (tseries). Além dos pacotes citados, utilizamos o pacote wavethresh, cujas funções são descritas no Livro Nason (2008). Utilizamos o pacote para obter bases de ondaletas para testar o modelo definido na Seção 4.

\section{C.2 Rotina para simular modelos com cointegração variando com o tempo}

Apresentamos a seguir a rotina utilizada para gerar os modelos simulados apresentados na Seção 5. Por meio da rotina, podemos simular todos os exemplos apresentados na Seção, 
bem como estimar os parâmetros de cada modelo e gerar os gráficos apresentados.

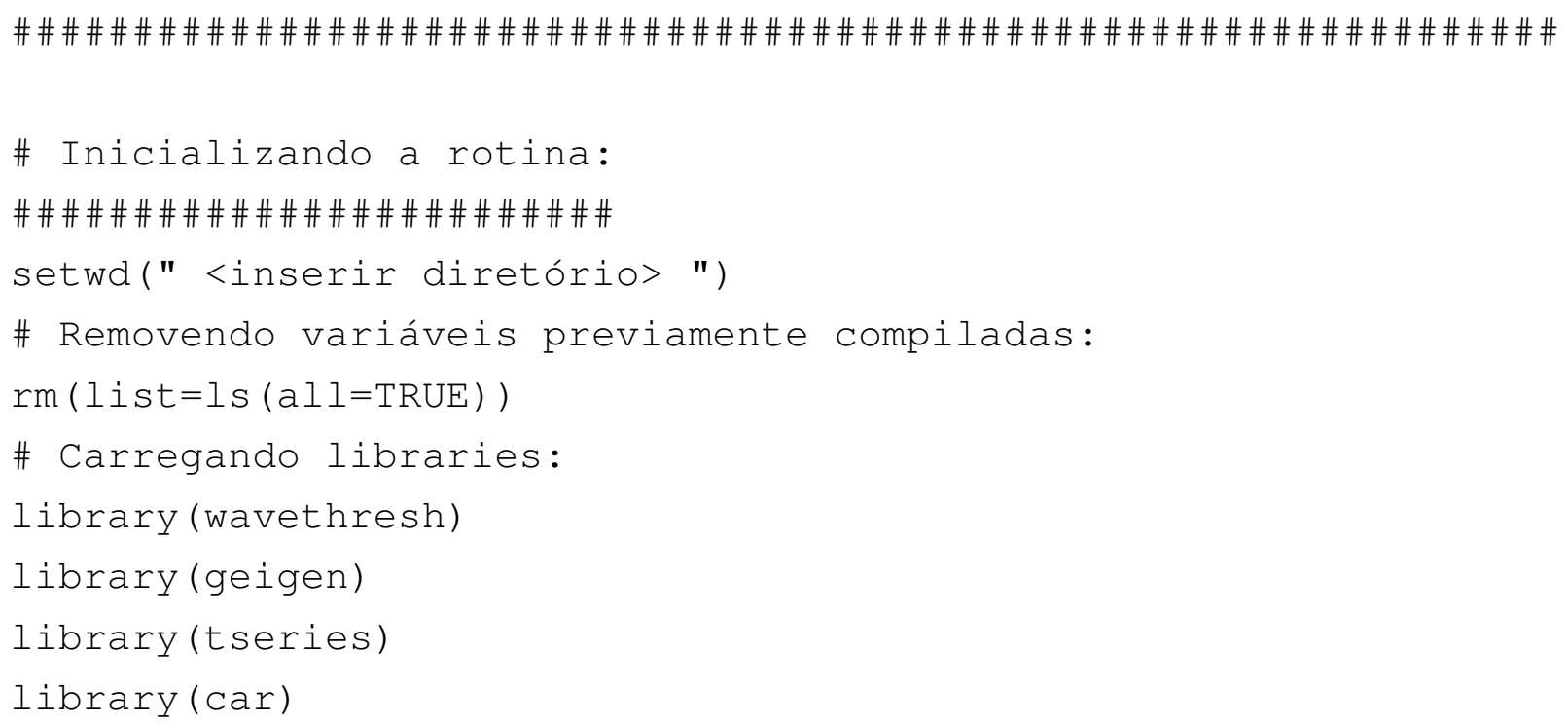




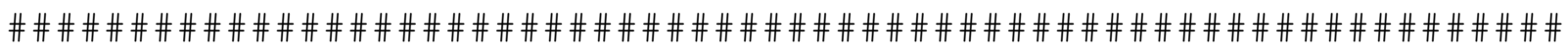

\# estruturas para O VEtor de cointegraÇÃo:

\# ESTRUTURA 01: Vetor de cointegração: beta_t = (beta_1, beta_2) $\rightarrow(\mathrm{kxr})$,

\# com beta_2 = (beta_21t,beta_22t), ou seja, uma mudança de regime ao longo do tempo

beta_21 $=-1$;

beta_22 $=-2$;

\#beta_23 $=-4$;

\#beta_24 = -2;

beta_2t $=c(r e p(\operatorname{beta} 21,(\mathrm{~T} / 2)), \operatorname{rep}(\operatorname{beta} 222,(\mathrm{~T} / 2)))$

$\#=c(r e p(\operatorname{beta} 21,(\mathrm{~T} / 4)), \operatorname{rep}(\operatorname{beta} 222,(\mathrm{~T} / 4)), \operatorname{rep}(\operatorname{beta} 23,(\mathrm{~T} / 2)))$ \#beta_2t $=c(\operatorname{rep}($ beta_21, $(\mathrm{T} / 4))$, rep $($ beta_22, $(\mathrm{T} / 4))$, rep (beta_23

$\left.,(\mathrm{T} / 4)), \operatorname{rep}\left(\operatorname{beta}{ }_{24},(\mathrm{~T} / 4)\right)\right)$

\# ESTRUTURA 02: decrescimento linear de beta_2t em $(0,1]$

\#beta_2t $=-((1 / \mathrm{T}) \star \mathrm{c}(1: \mathrm{T}))$

\# EStRUtURA 03: decrescimento logarítimico de beta_2t em $(0,1]$ \#beta_2t $=-\log ((1 / \mathrm{T}) \star \mathrm{c}(1: \mathrm{T}))$

\# EStRUtuRA 04: comportamento beta_2t em $(0,1]$ : função seno \#beta_2t $=-\sin ((40 / T) * C(1: T))$

\#plot $(1: T$, beta_2t, type="l")

\# estrutura 05: comportamento beta_2t em $(0,1]$ : mix de funções \#beta_2t $=c(r e p($ beta_21, $(\mathrm{T} / 4)),-1-((1 / \mathrm{T}) \star c(1:(\mathrm{T} / 4))), \operatorname{rep}($

beta_22,( $\mathrm{T} / 2))$ )

\#plot $(1:(T)$, beta_2t, type="l $)$

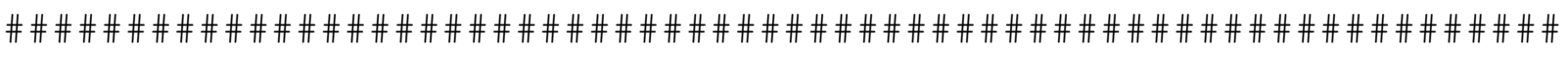

\# Simulando séries cointegradas:

\# Dim. da série simulada, consid. amostra inicial:

$\mathrm{TT}=\mathrm{T}+\mathrm{P}$ 
\# Vetor beta_2 variando com o tempo:

beta_2sim $=c(r e p($ beta_2t $[1], p)$, beta_2t $)$

\# Distribuição dos resíduos: $\mathrm{N}(0,1)$ independentes

$\mathrm{u}=\operatorname{matrix}(\operatorname{rnorm}(\mathrm{TT}, 0,1), \mathrm{TT}, 1)$

$\mathrm{V}=\operatorname{matrix}(\operatorname{rnorm}(\mathrm{TT}, 0,1), \mathrm{TT}, 1)$

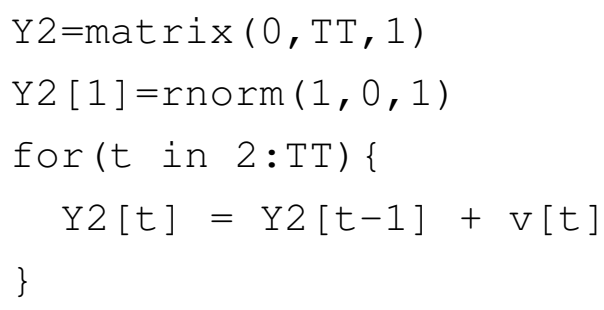




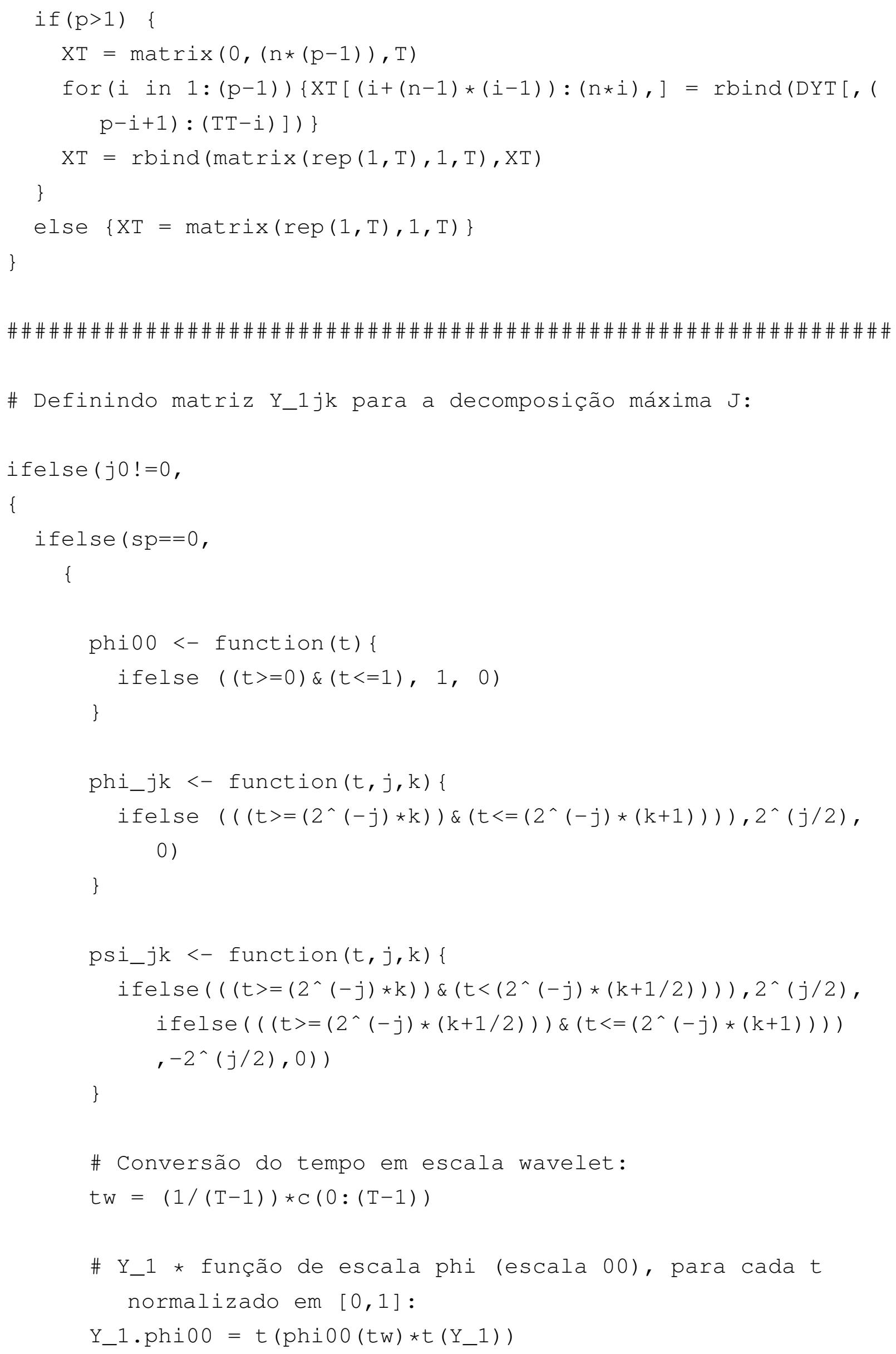




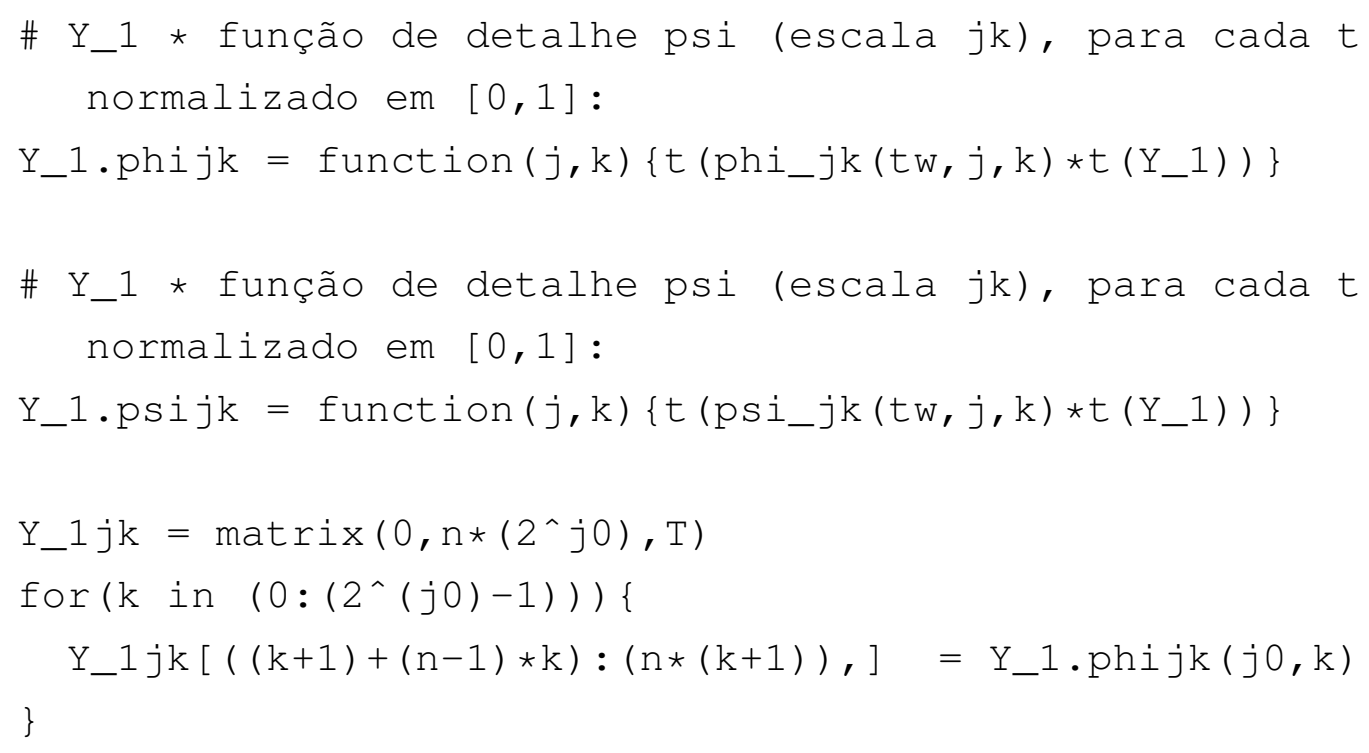




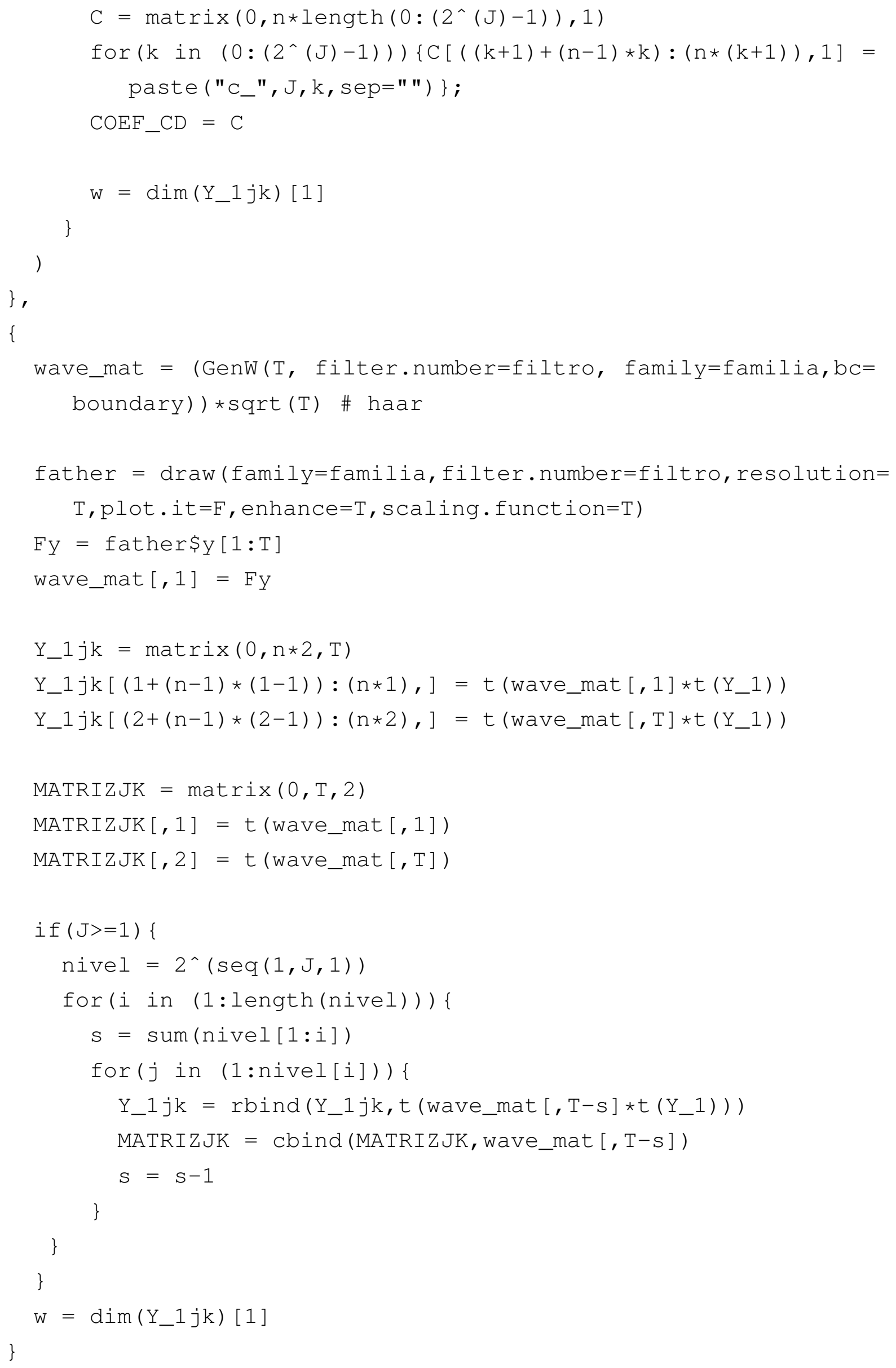


)

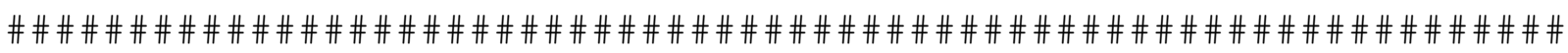

\# Preparando matrizes para a opção de threshold por níveis:

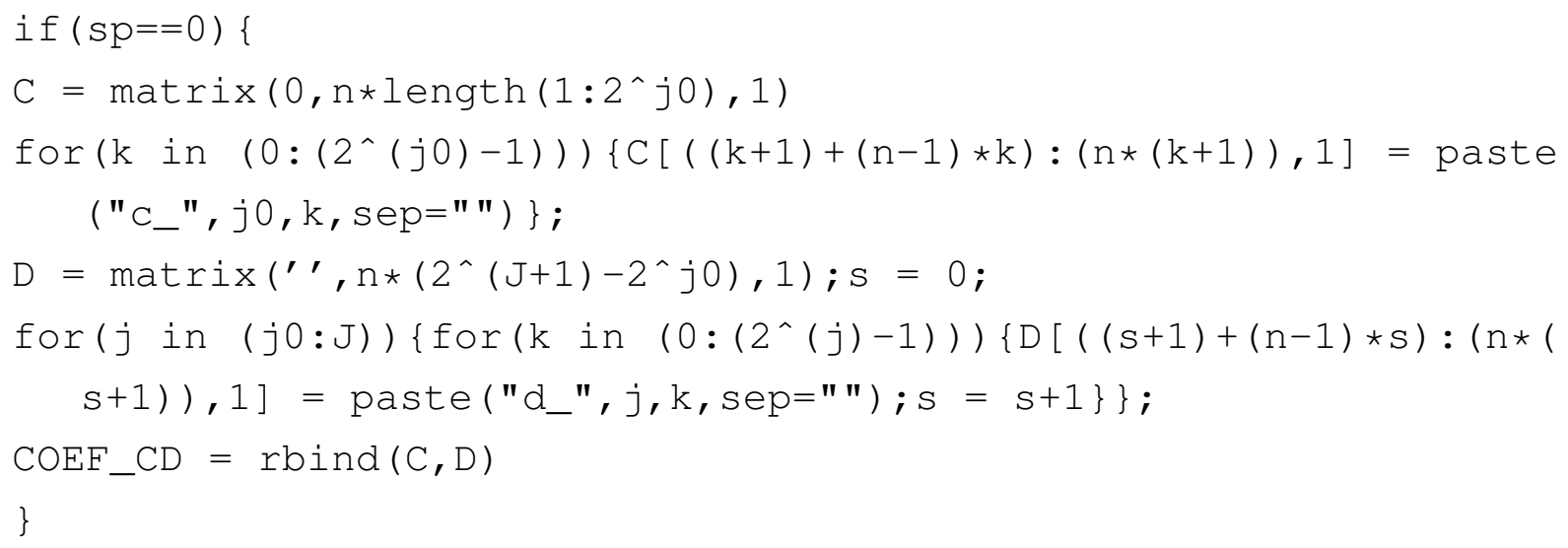




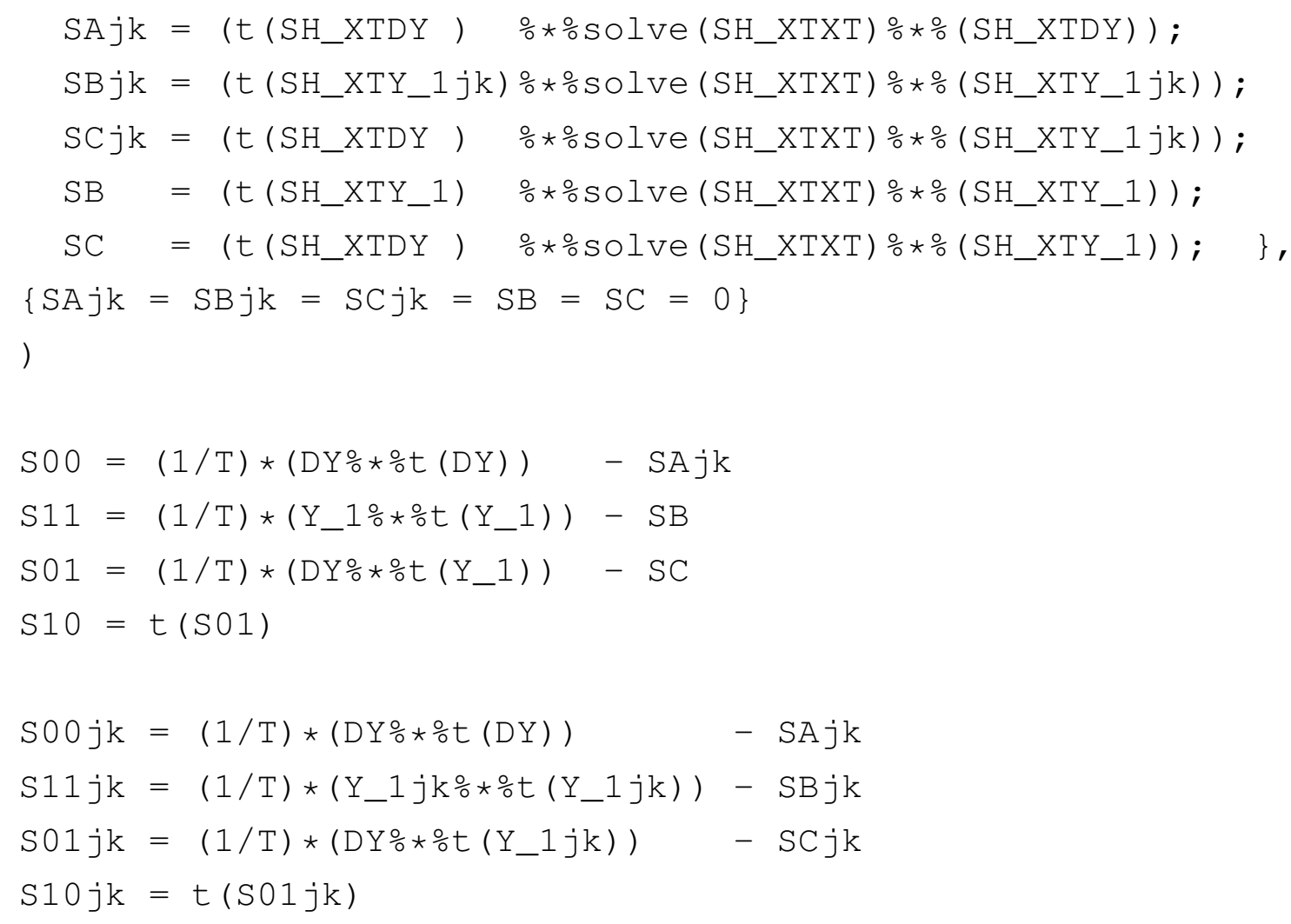

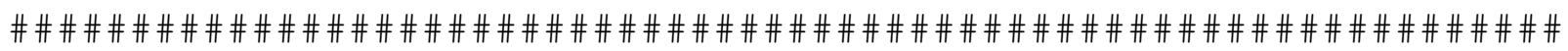

\# Resolvendo problema do autovalor e autovetor do modelo VEC:

\# Matriz S11^(-1/2)

S1leig <- eigen (S11)

S11_12 <- S11eig\$vectors $*$ \%diag(sqrt(1/S11eig\$values)) *oolve( slleig\$vectors)

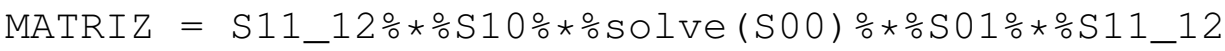

eigval = eigen (MATRIZ) \$values

eigvec = eigen (MATRIZ) \$vectors

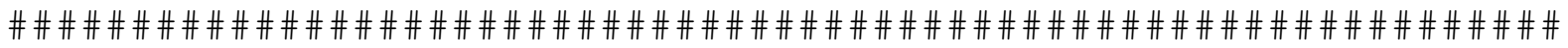
\# Resolvendo problema do autovalor e autovetor do modelo:

\# Matriz S11^(-1/2)

Slleigjk <- eigen(S11jk)

S11_12jk <- S1leigjk\$vectors\%*\%diag(sqrt(1/S11eigjk\$values)) \%*\% solve (Slleigjksvectors) 
MATRIZjk $=S 11$ S12jk\%*

eigvaljk = eigen (MATRIZjk) \$values

eigvecjk = eigen(MATRIZjk) \$vectors

\# Estimativa de M.V. de W_jk:

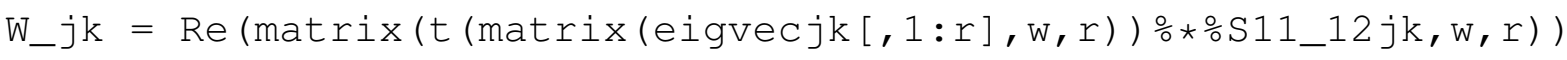
rownames $\left(W_{-} j k\right)=$ rownames $\left(Y_{-} 1 j k\right)$

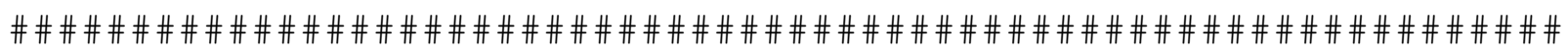

\# Thresholding: Threshold universal

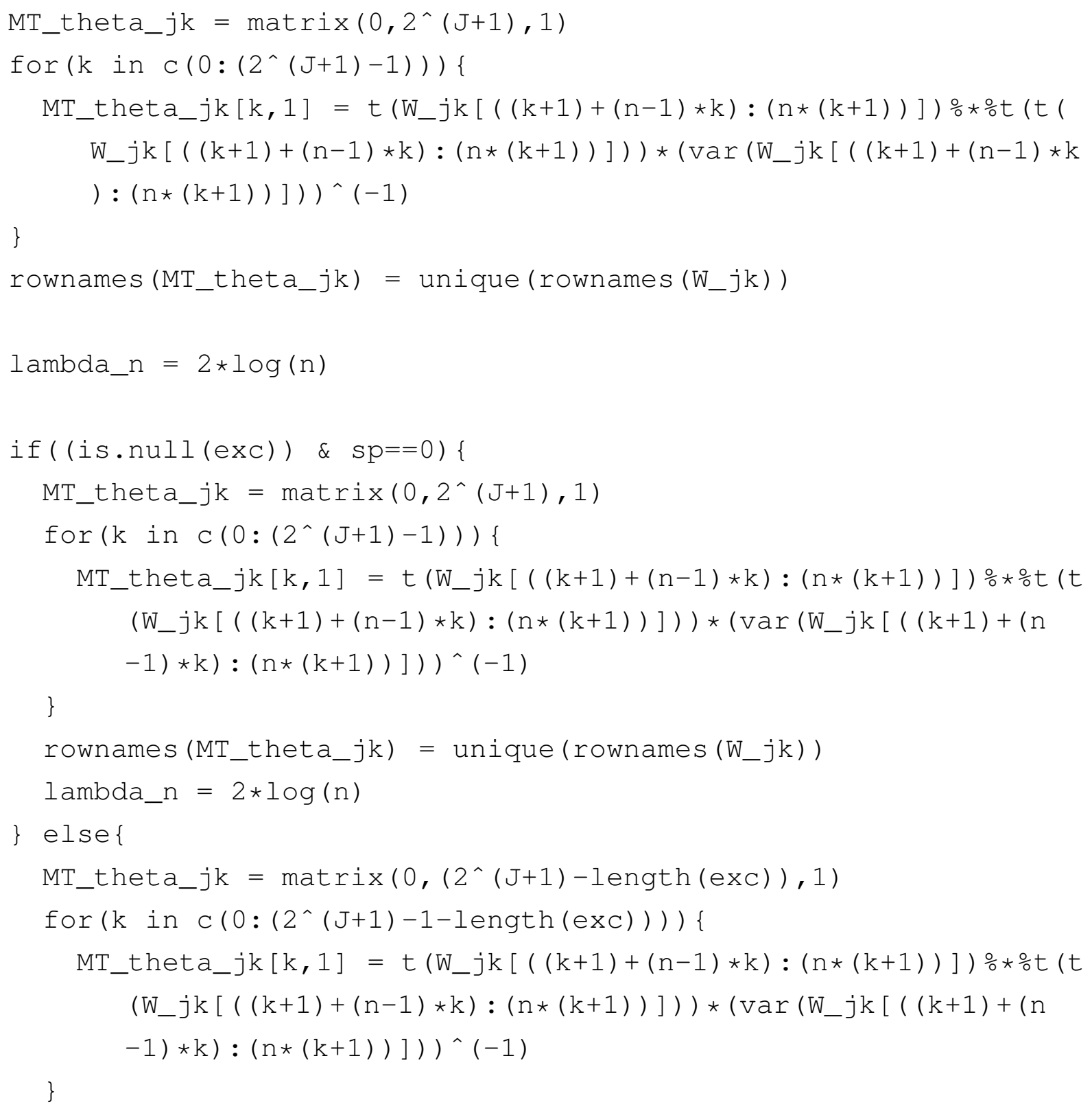




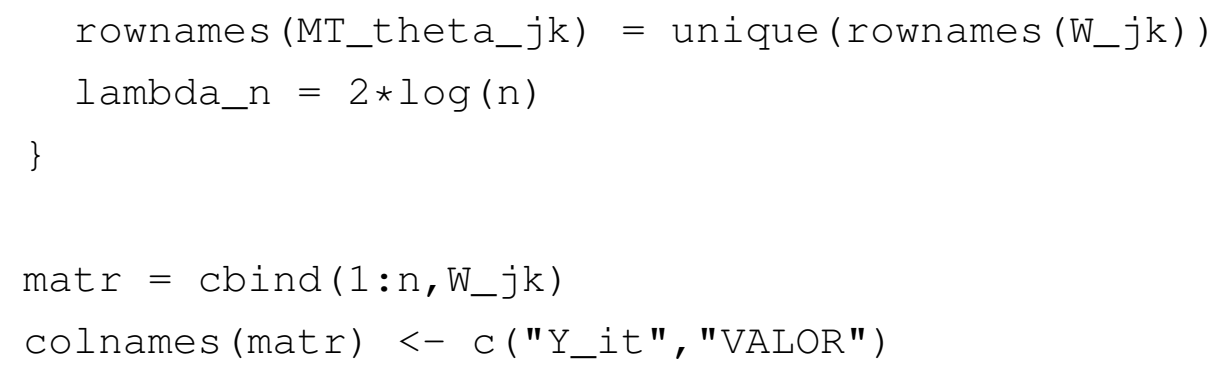




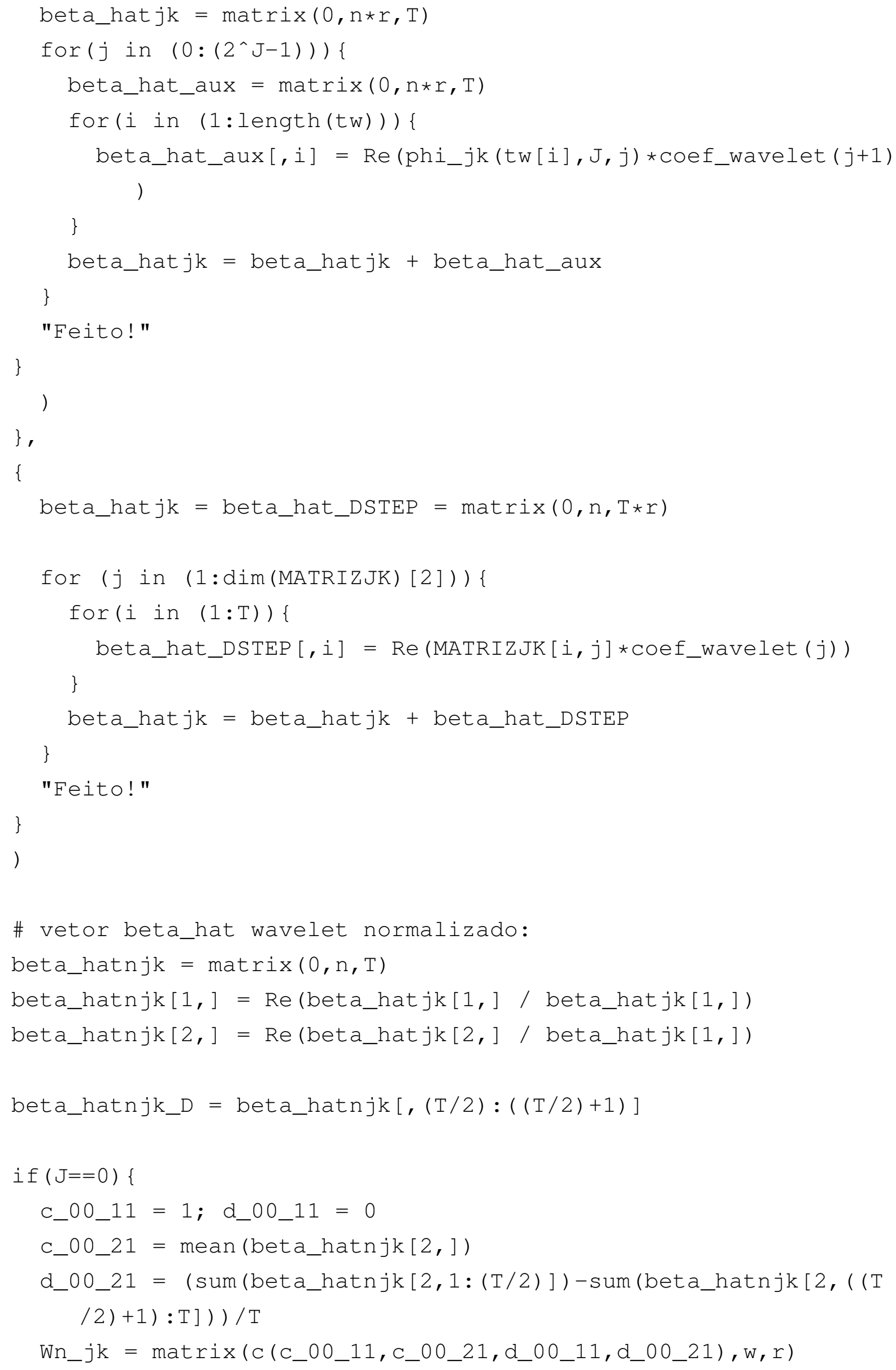




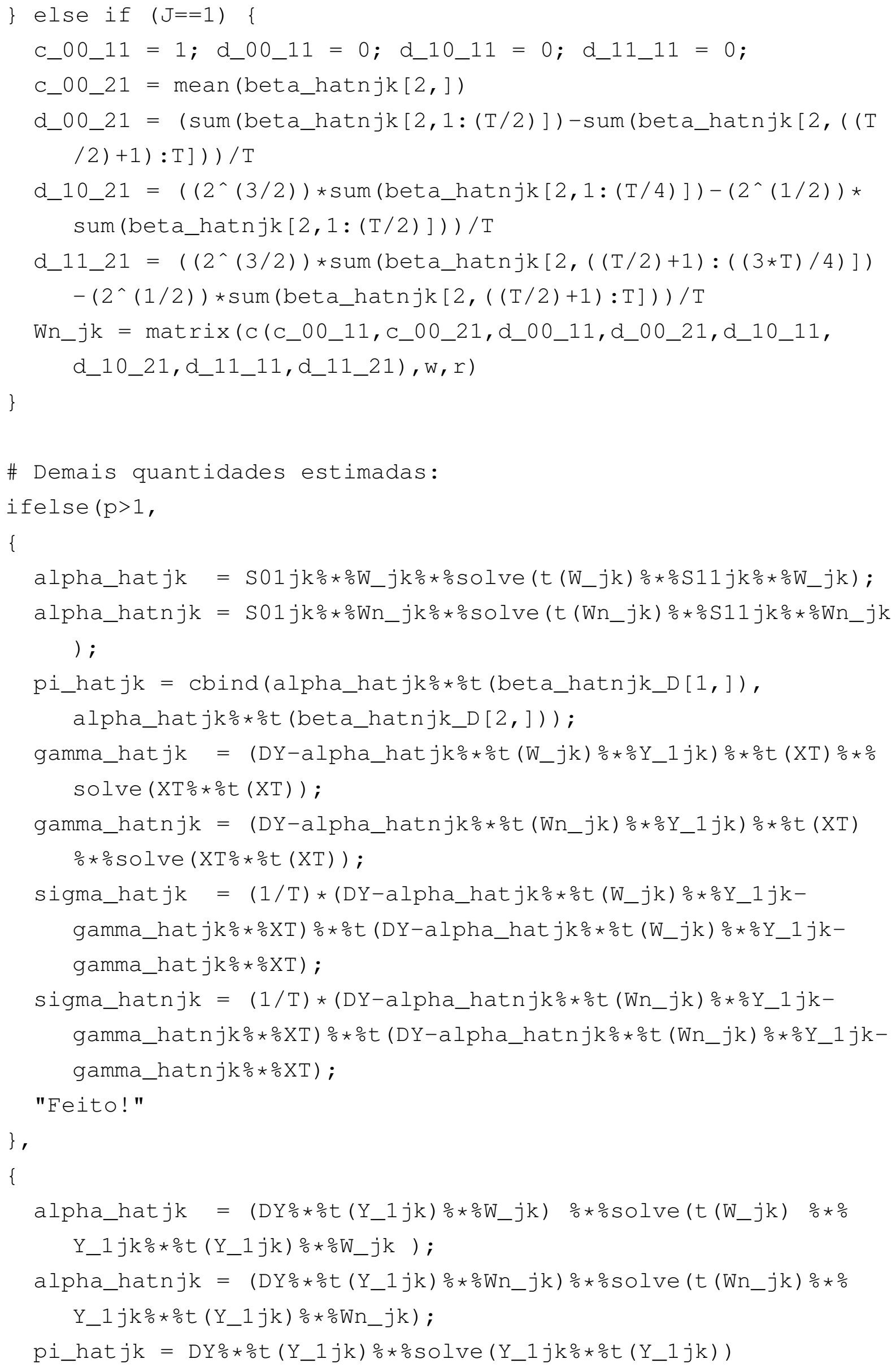




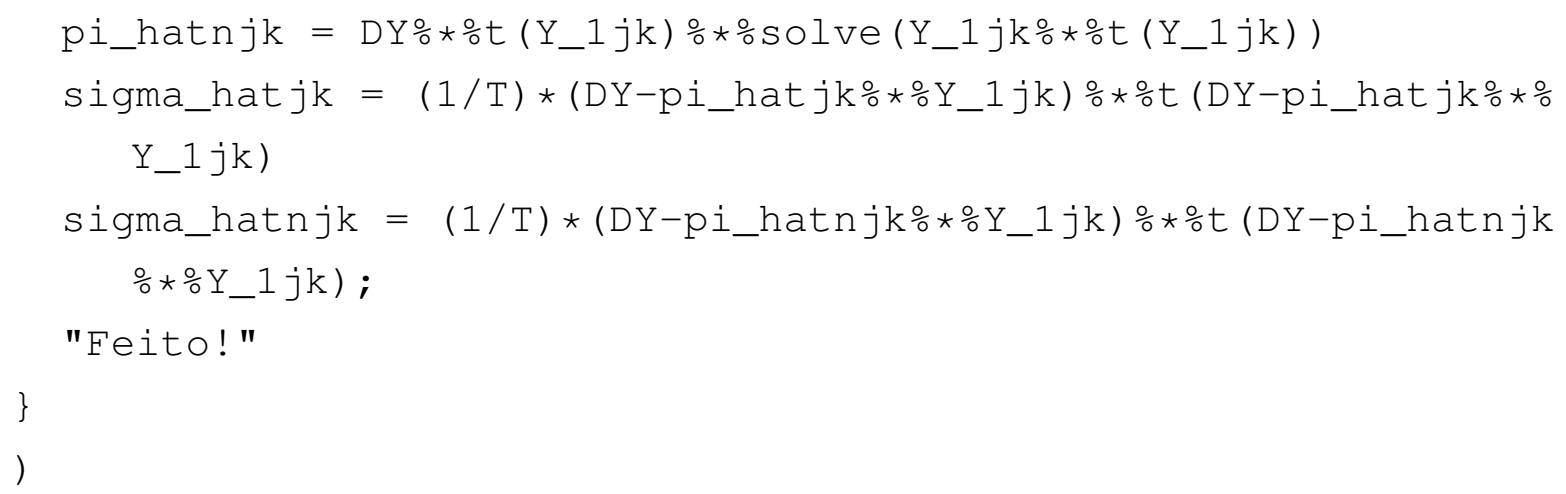

\# Relações de cointegração (RC) variando com o tempo: Estimadas (E) e Teóricas ( T)

$\operatorname{par}(\operatorname{mfrow}=\mathrm{c}(1,1), \mathrm{cex}=1.3, \operatorname{mar}=\mathrm{c}(4.8,5.2,1.5,1.5))$

titulo01 = expression(paste("Valores teóricos e estimados para

", beta[2][t])) 
plot.ts (beta_2t, lty $=1,1 \mathrm{wd}=2, \mathrm{xlab}=$ "tempo t $", y \lim =\mathrm{c}(-2.3,-0.9)$,

ylab=expression (hat (beta) [2] [t]), col=1, main=titulo01)

lines (beta_hatnjk $[2],, 1 t y=4, \operatorname{col}=4,1 \mathrm{wd}=3$ )

leg.txt01 = c ("TEÓRICO", "ESTIMADO")

legend ("bottomleft", leg.txt 01, bty $=" n ", \operatorname{col}=c(1,4), 1$ ty $=c(1,4), 1 w d$

$=c(2,3)$, horiz=T, cex=1)

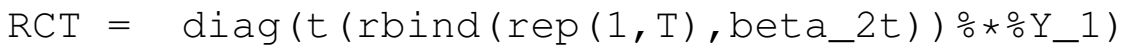

$\mathrm{RCE}=\operatorname{diag}\left(\mathrm{t}\left(\right.\right.$ beta_hatjk) $\left.\% * \% \mathrm{Y} \_1\right)$

RCE2 = diag (t (beta_hatnjk) $\left.\% * \frac{\circ}{0}{ }_{1}\right)$

$\operatorname{par}(\operatorname{mfrow}=\mathrm{c}(1,1), \mathrm{cex}=1.3,1 \mathrm{wd}=2, \operatorname{mar}=\mathrm{c}(4.8,4.8,1.5,1.5))$

titulo02 = expression(paste("Resíduos de cointegração

normalizados (", beta $\left.\left.[t]^{\wedge}\{1\}, Y[t-1], "\right) "\right)$ )

ts.plot $(\mathrm{RCT}, \mathrm{Col}=1,1 \mathrm{ty}=1,1 \mathrm{wd}=2, \mathrm{ylim}=\mathrm{C}(\min ((\mathrm{RCT}-1 * \operatorname{var}(\mathrm{RCT})),(\mathrm{RCE}$

$-1 \star \operatorname{var}(R C E)))$, $\max (R C T, R C E)), y l a b="$ ", xlab="tempo" , main=

titulo02)

lines ( $\mathrm{RCE} 2, \operatorname{col}=4,1 \mathrm{ty}=4,1 \mathrm{wd}=3$ )

$\operatorname{leg} 1=\operatorname{expression}\left(\right.$ paste $\left(\right.$ beta $\left.\left.[t]^{\wedge}\{1\} * Y[t-1]\right)\right)$

leg2 = expression (paste (hat (beta) $\left.[t]^{\wedge}\{1\} * Y[t-1]\right)$ )

$\operatorname{leg} \cdot \operatorname{txt} 00=\mathrm{c}(\operatorname{leg} 1, \operatorname{leg} 2)$

legend ("bottom", leg.txt00, bty $=" n ", \operatorname{col}=\mathrm{c}(1,4), 1 \mathrm{ty}=\mathrm{c}(1,4), 1 \mathrm{wd}=\mathrm{c}$

$(2,3)$, horiz = TRUE, $\operatorname{cex}=1)$

$\operatorname{par}(\operatorname{mfrow}=\mathrm{c}(1,1), \mathrm{cex}=1.3,1 \mathrm{wd}=2, \operatorname{mar}=\mathrm{c}(4.8,4.8,1.5,1.5))$

titulo02 = expression(paste("Resíduos de cointegração não

normalizados (", beta $\left.\left.\left.[t]^{\wedge}\{1\}, Y[t-1], "\right) "\right)\right)$

ts.plot $(R C T, C o l=1, y l i m=C(\min ((\operatorname{RCT}-1 * \operatorname{var}(R C T)),(R C E-1 * \operatorname{var}(R C E)))$

, $\max (\mathrm{RCT}, \mathrm{RCE})), 1 \mathrm{t}_{\mathrm{y}}=1, \mathrm{y} l \mathrm{ab}="$ ", $\mathrm{xl} a \mathrm{~b}=$ "tempo", main=titulo02)

lines $(\mathrm{RCE}, \mathrm{COl}=4,1 \mathrm{ty}=4,1 \mathrm{wd}=2)$

$\operatorname{leg} 1=\operatorname{expression}(\operatorname{paste}(\mathrm{Y}[1][t-1]-$ beta $[2][t] * \mathrm{Y}[2][t-1])$ )

leg2 = expression(paste (Y[1] [t-1]-hat (beta) [2] [t-1]*Y[2][t-1]))

$\operatorname{leg} \cdot \operatorname{txt} 00=c(\operatorname{leg} 1, \operatorname{leg} 2)$

legend ("bottomleft", leg.txt 00,1 ty $=c(1,4)$, bty $=" n ", \operatorname{col}=c(1,4)$,

horiz = TRUE, cex=1)

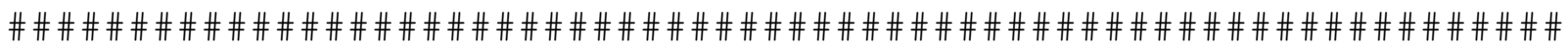

\# Resíduos de cointegração: Testando a estacionariedade 
\# Augmented Dickey-Fuller Test: alternativa é estacionária adf.test (RCE)

\# Teste Phillips-Perron de raiz unitária. Hipótese alternativa: Série estacionária.

$\mathrm{pp}$. test (RCE)

\# Teste KPSS: for the null hypothesis that $\mathrm{x}$ is level or trend stationary

kpss.test (RCE)

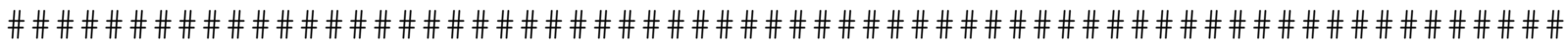

\# Análise de resíduos do modelo:

\# Resíduos normalizados:

ifelse $(\mathrm{p}>1$,

$\{$ ut $=$ DY - alpha_hatjk\%*\%diag $(t($ beta_hatnjk $) \% * \%$ _1 $)-$

gamma_hatjk*․ XT; "Done! "\},

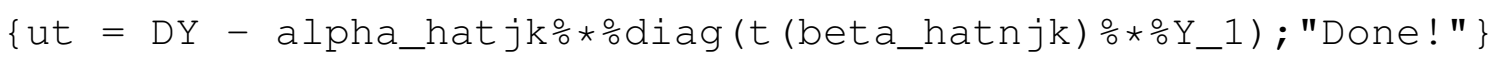

)

$\operatorname{par}(\operatorname{mfrow}=\mathrm{c}(3,2), \operatorname{mar}=\mathrm{c}(4.8,5.2,1.5,1.5), \mathrm{cex} .1 \mathrm{ab}=1.3)$

plot.ts (ut [1, ],ylab=expression (paste (hat (u) [1] [t] )), xlab="tempo

")

plot.ts (ut [2,],ylab=expression (paste (hat (u) [2] [t])), xlab="tempo

")

qqP lot (ut $[1],, 1 \mathrm{wd}=1$, distribution="norm", envelope=.95, col=1, col.

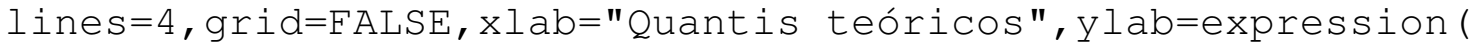

paste ("Quantis amostrais de ", hat(u)[1][t])))

qqline (ut $[1]$,

qqP lot (ut $[2],, 1 w d=1$, distribution="norm", envelope=.95, col=1, col .

lines=4,grid=FALSE, xlab="Quantis teóricos",ylab=expression (

paste ("Quantis amostrais de ", hat(u)[2][t])))

qqline (ut $[2$,$] )$

pacf (ut[1,], ylab=expression(paste("FACP de ", hat(u)[1][t])))

pacf (ut[2,], ylab=expression (paste("FACP de ", hat(u)[2][t]))) 
\# Teste de normalidade:

shapiro.test (ut $[1$,$] )$

shapiro.test (ut $[2$,$] )$

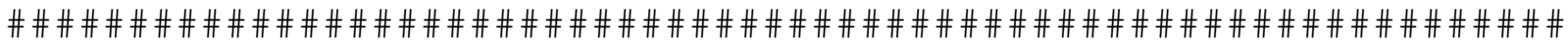

\# Parâmetros estimados:

beta_hatnjk $[,(\mathrm{T} / 2):((\mathrm{T} / 2)+1)]$

if $(\mathrm{J}<=1)\left\{\mathrm{Wn} \_j \mathrm{k}\right\}$ else $\left\{\mathrm{W}_{-} j \mathrm{k}\right\}$

alpha_hatjk

pi_hatjk

if $(p>1)$ \{print (gamma_hatjk) \}

sigma_hatjk

LR_TVCjk

\# Máx Log-verossimilhança:

$\max \_L V=-(T / 2) * \operatorname{sum}(\log (1-\operatorname{eigval}[1: r]))-(T / 2) * \log (\operatorname{det}(\operatorname{S} 00 j k))$

$-((\mathrm{n} * \mathrm{~T}) / 2) * \log (2 * \mathrm{pi})-((\mathrm{n} * \mathrm{~T}) / 2)$

max_LV

\# Coeficientes wavelet estimados:

ifelse(! (is.null (exc)),

\{print(matr); paste('Modelo reduzido! Excluídos os níveis:

', list (exc))\},

\{print(matr) ;'Modelo completo!'

\}

)

\# Threshold universal:

if $(\mathrm{J}>0)\{$

$\operatorname{par}(\operatorname{mfrow}=\mathrm{c}(1,1), \operatorname{mar}=\mathrm{c}(4.8,5.5,1.5,1.5), \mathrm{cex} .1 \mathrm{ab}=1.8,1 \mathrm{wd}=1)$

plot (MT_theta_jk, type $={ }^{\prime} \mathrm{p}^{\prime}, \mathrm{col}=1, \mathrm{ylim}=\mathrm{C}(0,9), \mathrm{ylab}=\operatorname{expression}($

paste(theta[j][k],' para níveis ',' ', d[j][k])), xlab='

coeficientes', main="Threshold Universal - contexto

multivariado") \# xaxt $=$ ' $\mathrm{n}$ ', $\mathrm{xlab}=$ "coeficientes de ondaletas" text (MT_theta_jk, unique (colnames (MATRIZJK)), pos=3, cex=0.8)

lines (matrix (lambda_n, 2^(J+1), 1), col=4, lty=2)

lines (matrix $\left.\left(0,2^{\wedge}(\mathrm{J}+1), 1\right), \mathrm{col}=1,1 \mathrm{ty}=6\right)$ \} 
\# Relações de cointegração (RC) variando com o tempo: Estimadas

(E) e Teóricas $(\mathrm{T})$

$\operatorname{par}(\operatorname{mfrow}=\mathrm{c}(1,1), \mathrm{cex}=1.3, \operatorname{mar}=\mathrm{c}(4.8,5.2,1.5,1.5))$

titulo01 = expression(paste("Valores teóricos e estimados para

", beta[2][t]))

plot.ts (beta_2t, lty $=1,1 \mathrm{wd}=2, \mathrm{xlab}=$ "tempo $t$ ", ylim=c $(-2.3,-0.9)$,

ylab=expression (hat (beta) [2] [t]), col=1, main=titulo01)

lines (beta_hatnjk $[2],, l t y=4, \operatorname{col}=4,1 \mathrm{wd}=3$ )

leg.txt01 = c ("TEÓRICO", "ESTIMADO")

legend ("bottomleft", leg.txt $01, \mathrm{bty}=" \mathrm{n} ", \operatorname{col}=\mathrm{c}(1,4), 1$ ty $=\mathrm{c}(1,4), 1 \mathrm{wd}$ $=c(2,3)$, horiz $=\mathrm{T}, \mathrm{cex}=1)$ 


\section{Referências Bibliográficas}

Baltagi, B. H. (2008). Econometrics. Springer Texts in Business and Economics, fourth edition.

Bierens, H. J. e Martins, L. F. (2010). Time-varying cointegration. Econometric Theory, 26:1453-1490.

Bruce, A. e Gao, H.-Y. (1996). Applied wavelet analysis with S-plus. Springer-Verlag New York, Inc.

Cheung, Y.-W. e Lai, K. S. (1993). Long-run purchasing power parity during the recent float. Journal of international Economics, 34(1-2):181192.

Donoho, D. L. (1993). Unconditional bases are optimal bases for data compression and for statistical estimation. Applied and computational harmonic analysis, 1(1):100-115.

Donoho, D. L., Johnstone, I. M., Kerkyacharian, G., e Picard, D. (1995). Wavelet shrinkage: asymptopia? Journal of the Royal Statistical Society. Series B (Methodological), pages 301-369.

Donoho, D. L. e Johnstone, J. M. (1994). Ideal spatial adaptation by wavelet shrinkage. Biometrika, 81(3):425-455.

Downie, T. e Silverman, B. (1998). The discrete multiple wavelet transform and thresholding methods. IEEE Transactions on Signal Processing, 46(9):2558-2561. 
Engle, R. F. e Granger, C. W. (1987). Co-integration and error correction: representation, estimation, and testing. Econometrica: Journal of the Econometric Society, pages 251-276.

Falk, B. e Wang, C.-H. (2003). Testing long-run ppp with infinite-variance returns. Journal of Applied Econometrics, 18(4):471-484.

Granger, C. W. (1986). Developments in the study of cointegrated economic variables. Oxford Bulletin of economics and statistics, 48(3):213-228.

Hamilton, J. D. (1994). Time series analysis. Princeton University Press, first edition.

Hannan, E. J. e Quinn, B. G. (1979). The determination of the order of an autoregression. Journal of the Royal Statistical Society. Series B (Methodological), pages 190-195.

Hayashi, F. (2000). Econometrics. Princeton University Press, first edition.

Hendry, D. F. e Juselius, K. (2000). Explaining cointegration analysis: Part 1. The Energy Journal, 21:1-42.

Hendry, D. F. e Juselius, K. (2001). Explaining cointegration analysis: Part II. The Energy Journal, 22:75-120.

IMF (2016). International financial statistics.

Johansen, S. (1988). Statistical analysis of cointegration vectors. Journal of economic dynamics and control, 12(2):231-254.

Johansen, S. (1995). Likekihood-based inference in cointegrated vector autoregressive models - Advanced texts in Econometrics. Oxford University Press, first edition.

Lutkepohl, H. (2005). New Introduction to multiple time series analysis. Springer, First edition.

Mallat, S. (1999). A wavelet tour of signal processing. Academic press. 
Martins, L. F. (2016). Bootstrap tests for time varying cointegration. Econometric Reviews, pages 1-18.

Martins, L. F. e Gabriel, V. J. (2013). Time-varying cointegration, identification, and cointegration spaces. Studies in Nonlinear Dynamics and Econometrics, 17(2):199-209.

Martins, L. F. F. d. (2005). Structural changes in nonstationary time series econometris: time varying cointegration and modeling catastrophic events.

Morettin, P. A. (2011). Econometria financeira - Um curso em séries temporais financeiras. Blucher, segunda edição.

Morettin, P. A. (2014). Ondas e ondaletas: da análise de Fourier à análise de ondaletas de séries temporais. EDUSP.

Nason, G. (2008). Wavelet methods in statistics with R. Springer.

Park, J. Y. e Hahn, S. B. (1999). Cointegrating regressions with time varying coefficients. Econometric Theory, 15(05):664-703.

Patel, J. (1990). Purchasing power parity as a long-run relation. Journal of Applied Econometrics, 5(4):367-379.

Phillips, P. C. e Perron, P. (1988). Testing for a unit root in time series regression. Biometrika, 75(2):335-346.

R-project (2016). R-project. https://www.r-project.org/about.html. acesso em 01/05/2016.

Rogoff, K. (1996). The purchasing power parity puzzle. Journal of Economic Literature, pages 647-668.

Said, S. E. e Dickey, D. A. (1984). Testing for unit roots in autoregressivemoving average models of unknown order. Biometrika, 71(3):599-607.

Sen, P. K., Singer, J. M., e de Lima, A. C. P. (2010). From finite sample to asymptotic methods in statistics. Cambridge University Press. 
Shapiro, S. S. e Wilk, M. B. (1965). An analysis of variance test for normality (complete samples). Biometrika, 52(3-4):591-611.

Shumway, R. H. e Stoffer, D. S. (2011). Time series analysis and its applications: with $R$ examples. Springer.

Strela, V., Heller, P. N., Strang, G., Topiwala, P., e Heil, C. (1999). The application of multiwavelet filterbanks to image processing. Image Processing, IEEE Transactions on, 8(4):548-563.

Taylor, A. M. e Taylor, M. P. (2004). The purchasing power parity debate. The Journal of Economic Perspectives, 18(4):135-158.

Tsay, R. S. (2010). Analysis of Financial Time Series. John Wiley \& Sons.

Tsay, R. S. (2013). Multivariate time series analysis: with $R$ and financial applications. John Wiley \& Sons.

Tso, M.-S. (1981). Reduced-rank regression and canonical analysis. Journal of the Royal Statistical Society. Series B (Methodological), pages 183-189.

Zivot, E. e Wang, J. (2006). Modeling financial time series with S-PLUS®, volume 191. Springer. 